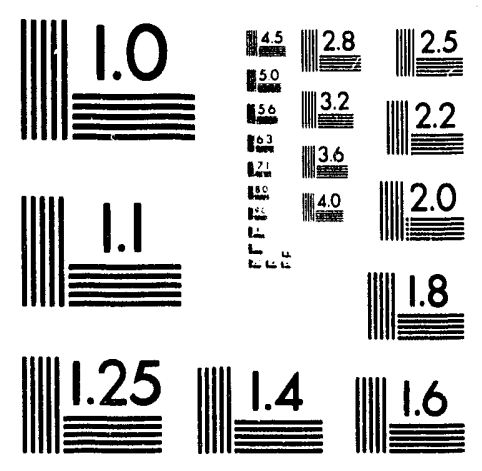



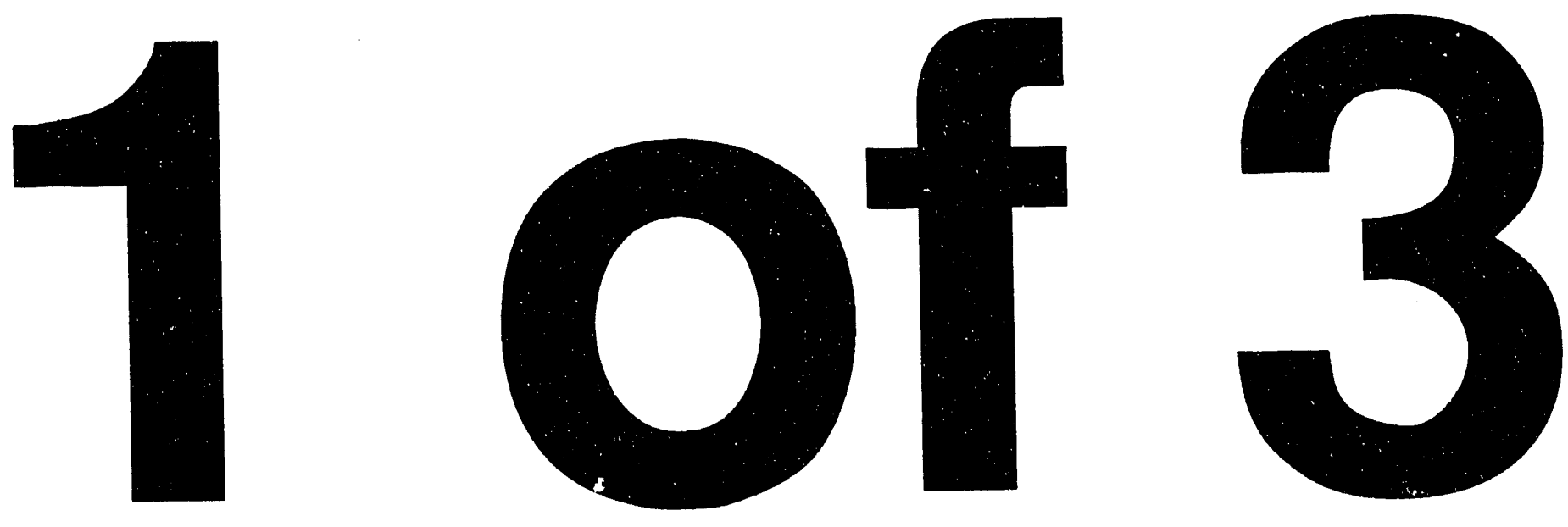
UCRL-LR-113984

Distribution Category UC-426

\section{Behavior of the Particle Transport Coefficients Near the Density Limit in MTX}

Michael Martin Marinak

(Ph.D. Thesis)

Manuscript date: April 1993

LAWRENCE LIVERMORE NATIONAL LABORATORY

University of California - Livermore, California 99551

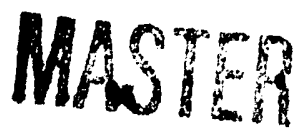

DISTRIBUTION OF THIS DOCUMENT IS UNLIMITED 


\title{
Behavior of the Particle Transport Coefficients Near the Density Limit in MTX
}

by

\author{
Michael Martin Marinak
}

B. S. (University of California at Berkeley) 1987

A dissertation submitted in partial satisfaction of the requirements for the degree of

Doctor of Philosophy

in

Engineering-Nuclear Engineering

in the

GRADUATE DIVISION

of the

UNIVERSITY of CALIFORNIA at BERKELEY

Committee in charge:

Professor Keith I. Thomassen, Chair

Professor Edward C. Morse

Professor Allan J. Lichtenberg

1993 
The dissertation of Michael Martin Marinak is approved:
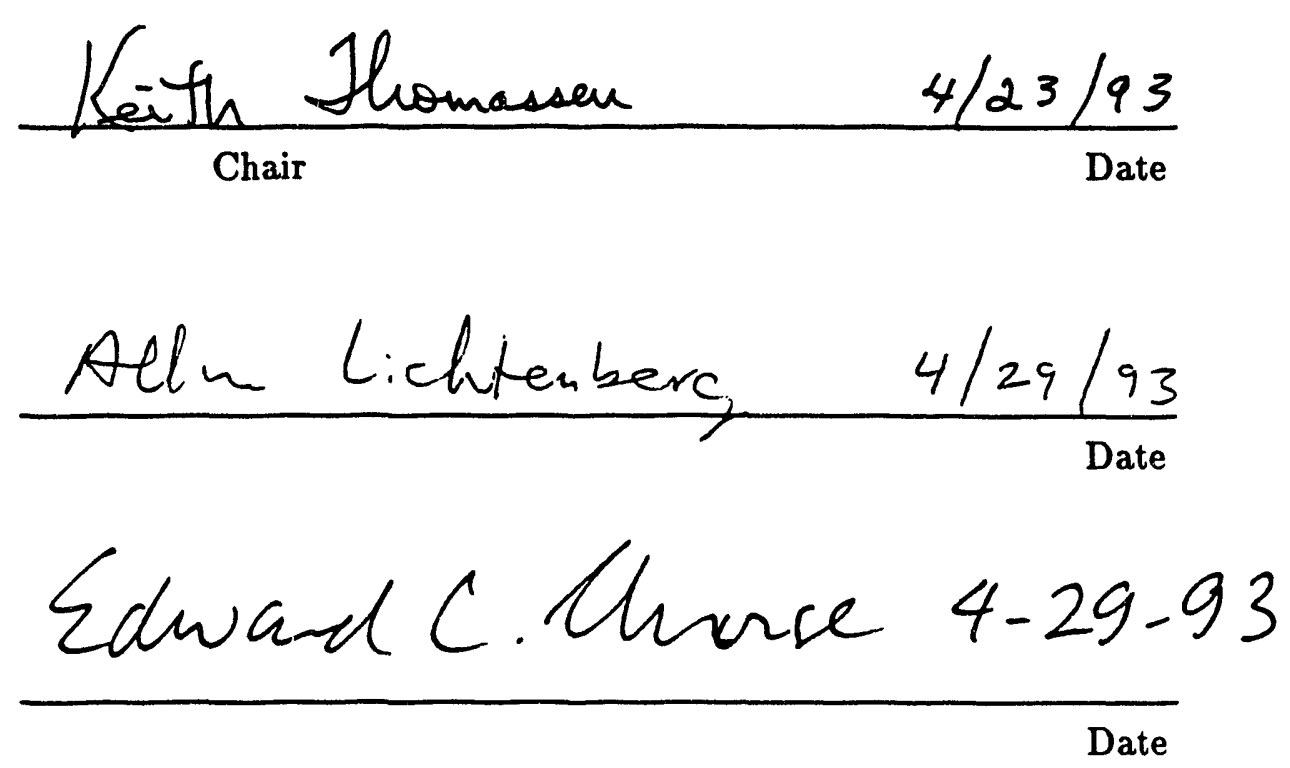

University of California at Berkeley

1993 


\author{
Abstract \\ Behavior of the Particle Transport Coefficients \\ Near the Density Limit in MTX \\ by \\ Michael Martin Marinak \\ Doctor of Philosophy in Engineering-Nuclear Engineering \\ University of California at Berkeley \\ Professor Keith I. Thomassen, Chair
}

The perturbed particle transport coefficients were determined for a range of plasma conditions in the Alcator $\mathrm{C}$ tokamak, a component of the Microwave Tokamak Experiment (MTX), from analysis of density perturbations created in gas modulation experiments. Density measurements from a 15 chord far-infrared interferometer were sufficiently detailed to allow radial profiles of the transport coefficients to be resolved. Gas modulation experiments were carried out on plasmas over a range of relatively low currents and a wide variety of line-averaged densities, including values near the Greenwald density limit. With this technique the perturbed diffusion coefficient D and the perturbed convection velocity $\mathrm{V}$ can be determined simultaneously. Measured profiles of $\mathrm{D}$ rise toward the outside of the plasma column in a manner generally similar to those determined previously for $\chi_{e, H P}$ from sawtooth heat pulse propagation. Values of $\mathrm{D}$ are typically smaller than those of $\chi_{e, H P}$ given for the same line-averaged densities by a factor of 2-5. Diffusion coefficients from a series of discharges at constant current showed little variation with density through most of the saturated ohmic confinement regime. At the Greenwald density limit threshold $\left(\bar{n}_{e}=\kappa \bar{J}\right)$ a dramatic increase occurred in both the perturbed convective and diffusive transport coefficients 
in the outer region of the plasma. The increases were most pronounced at the outermost range of the radii where coefficients were determined $(r / a=0.8)$, but were apparent over a region which extended well into the plasma interior. Density profiles maintained a similar shape near the density limit, congruous with the similar behavior of the transport coefficients. No dramatic deterioration was evident in the global energy confinement.

These increases in the transport coefficients occurred in regions of the plasma which had relatively low temperatures $\left(\mathrm{T}_{e}<250 \mathrm{ev}\right)$ and were in the Pfirsch-Schlüter collisionality regime. The variation of $D$ in this region of these high density plasmas compared well with the scaling given in a theory of resistivity-gradient-driven turbulence. The inverse dependences on the temperature and temperature gradient were key components of the model most closely associated with this result.

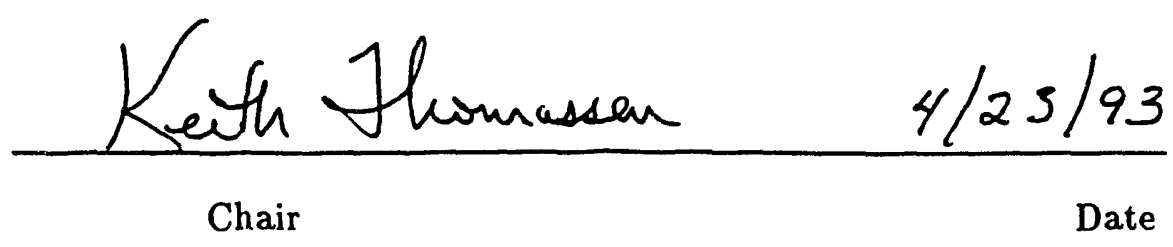




\section{Contents}

1 Introduction and Background 1

1.1 Density Limits . . . . . . . . . . . . . . . . . . . 1

1.2 Fuelling Limits . . . . . . . . . . . . . . . . . . 3

1.3 Density Limit Physics and Phenomena . . . . . . . . . . . 3

1.4 Summary of Experiment and Results . . . . . . . . . . . . 11

1.5 Outline of Thesis . . . . . . . . . . . . . . 15

2 Method of Experiment and Theory of Data Analysis 17

2.1 Particle Transport . . . . . . . . . . . . . . . 18

2.1.1 Previous Observations . . . . . . . . . . . . . . . 19

2.2 Consequences of Transport Coefficient Dependences on Plasma Param-

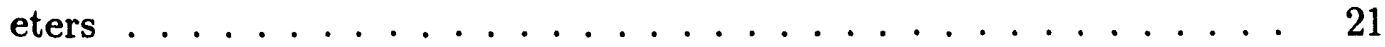

2.3 Experimental Considerations . . . . . . . . . . . . . . 24

2.3.1 Stability of Plasma Position . . . . . . . . . . . 27

2.3.2 Plasma Response to Gas Modulation . . . . . . . . . . . 29

3 Method of Data Analysis 33

3.1 Processing of FIR Signals $\ldots \ldots \ldots \ldots \ldots$

3.2 Equilibrium Simulation . . . . . . . . . . . . . . 35

3.3 Profile Determination . . . . . . . . . . . . . . . . . . 39

3.3.1 Refraction Corrections . . . . . . . . . . . . . . 42

3.3.2 Calculation of Chord Integrals . . . . . . . . . . . 44

3.4 Analysis in the Time Domain . . . . . . . . . . . . . 46

3.4.1 Spline Fit . . . . . . . . . . . . . . 46

3.4 .2 Matrix Inversion . . . . . . . . . . . . . . 48

3.4.3 Multidimensional Linear Regression Analysis . . . . . . . . . 49

3.5 Impact of Particle Source Term . . . . . . . . . . . . . . . . 49

3.6 Effect of Filtering and Influence of Spline Fit Parameters . . . . . . 55

3.7 Spectral Analysis Methods . . . . . . . . . . . . . . . . 58

3.8 Summary of Analysis Procedure . . . . . . . . . . . . 58

4 Experimental Results $\quad 59$

4.1 Density Profiles . . . . . . . . . . . . . . . . . . 61

4.2 Edge Asymmetries and Measured Density Profile Evolution . . . . . . 63

4.3 Trends Observed as Density Limit is Approached . . . . . . . . . 67 
4.3.1 Electron Temperature Profile Measurement . . . . . . . . . 72

4.3.2 Variation of Particle Transport Coefficients . . . . . . . . . 74

4.4 Density Limit at Higher $\mathrm{q} \ldots \ldots \ldots$

4.5 Relations Between Transport Coefficients . . . . . . . . . . . 79

4.6 Anomalous Inward Convection . . . . . . . . . . . . . . . 81

4.7 Results with Periodic Modulation . . . . . . . . . . . . . . 82

4.8 Comparison of Results form Spectral/Time Domain Analysis . . . . . 88

4.9 Significance of Model . . . . . . . . . . . . . . . . . . 89

4.9 .1 Error Propagation . . . . . . . . . . . . . 89

4.9.2 Significance of Temperature Perturbations $\ldots \ldots \ldots$

5 Comparison with Theory 95

5.1 Edge Turbulence Theories . . . . . . . . . . . . . . . . 96

5.2 Resistivity-Gradient-Driven Turbulence Theories . . . . . . . . . . . 98

5.2.1 Comparison of Transport Coefficients with RGDT Theory . . 104

5.2.2 Comparison with Trends in Global Energy Confinement Time 107

5.2.3 Tests of Assumptions Used in Reduced Braginskii Equations . 110

5.2 .4 Vorticity Decoupling Approximation . . . . . . . . 110

5.2 .5 Other Notes about the RGDT Theory . . . . . . . . . 112

5.3 Resistive Ballooning Theory . . . . . . . . . . . . . . . 114

5.4 Other Turbulence Theories . . . . . . . . . . . . . . . . . 116

5.5 Discussion . . . . . . . . . . . . . . . . 117

6 Comparison with Behavior on other Tokamaks 119

6.1 Location of Region of Confinement Deterioration in Plasma Profiles . 120

6.2 Relation to Edge Turbulence . . . . . . . . . . . . . . . . 122

6.2.1 Relation to Improved Confinement Modes . . . . . . . . . 123

6.3 Density Limit Behavior in TFTR . . . . . . . . . . . . . . . . 125

6.4 Relation of Transport Coefficients to Plasma Profiles on T-10 . . . . 128

6.5 Significance of Scrape-Off Layer Behavior . . . . . . . . . . . . . 130

6.6 Discussion . . . . . . . . . . . . . . . . . . . 131

7 Conclusions $\quad 134$

A Calculation of Refraction Angle $\quad 137$

B Spectral Form of Continuity Equation 140

B.1 Finite Difference Equations for Spectral Analysis . . . . . . . . . 140

B.2 Finite Difference Equations for Simulation of Periodic Modulation . . 141

C Equations for Analysis in the Time Domain 143

C.1 Equations for Simulation of Modulation Data . . . . . . . . . 144

D Technicalities of Experimental Hardware 146

D.1 Interferometer Design . . . . . . . . . . . . . . . . . . . 146

D.2 Gas Fuelling System . . . . . . . . . . . . . . . 147 
F Spectrai Analysis Methods $\quad 150$

F.1 Issues Associated with Finite Fourier Transform . . . . . . . . . 150

F.2 Spectral Quantities . . . . . . . . . . . . . . . . 153

F.3 Methodology . . . . . . . . . . . . . . . . 153

F.4 Complementary Spectral Analysis Method . . . . . . . . . . . 155

G Accuracy of Fit $\quad 157$

G.1 Statistical Tests of Models . . . . . . . . . . . . . . . 157

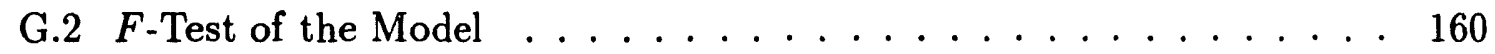

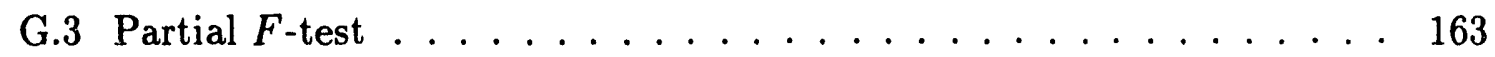

G.4 Error Propagation . . . . . . . . . . . . . . . . . . 164

H Model with Temperature Effects Explicitly Included 168

H.1 Biased Estimators . . . . . . . . . . . . . . . . . . . . . . 169

H.2 Statistical Tests of Extended Models . . . . . . . . . . . 172

I Outline of Resistivity-Gradient-Driven Turbulence Theories 175

I.1 RGDT with Impurity Dynamics Included . . . . . . . . . . . . . . 179

I.2 Neoclassical Resistivity-Gradient-Driven Turbulence . . . . . . . . . 180 


\section{List of Figures}

1.1 Density decay time (after pellet injection) versus $\bar{J} / \bar{n}$. (from Greenwald et al. [16]) . . . . . . . . . . . . . . . . . 7

1.2 Overview of the Microwave Tokamak Experiment . . . . . . . . . 12

1.3 Particle transport coefficients overlaid from $285 \mathrm{kA}$ shots with $\mathrm{n}_{e, 20}=$ $1.8,2.2,2.6,2.9,3.3 \ldots \ldots \ldots \ldots \ldots \ldots$

1.4 Scaling of resistivity gradient driven turbulence diffusion coefficients for plasmas of the $285 \mathrm{kA}$ density scan. . . . . . . . . . . 14

2.1 Amplitude and phases of chord measurements vs. radius with $\alpha=1$. . 26

2.2 Nearly constant plasma current during the plateau of shot $13552 \ldots 28$

2.3 Plasma position during shots 14034, 14040. A gas puff occurs at 260 msec. . . . . . . . . . . . . . . . . . . 28

2.4 Line-averaged density during periodic gas modulation on shot $13524 . \quad 29$

2.5 Gas valve flow with large amplitude, low duty cycle modulation. . . . 30

2.6 Line density during shot 13546 with a single gas puff modulation at 230 msec. . . . . . . . . . . . . . . . . . 31

3.1 FIR signal before and after fringe removal . . . . . . . . . . . 34

3.2 Superimposed FIR chord signals with and without filtering during gas

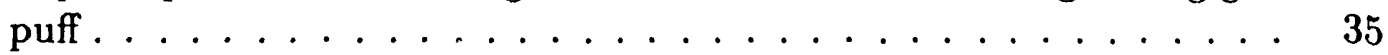

3.3 Cross section of the MTX Tokamak . . . . . . . . . . . . . 36

3.4 MTX surfaces of constant flux calculated by ACCOME. . . . . . . . 37

3.5 Plasma profiles used in the ACCOME simulation. . . . . . . . . . . 39

3.6 Effect of refraction on FIR ray geometry . . . . . . . . . . . . . . 43

3.7 Profile of particle source term for shot 14079 at $280 \mathrm{msec}$ calculated from ONETWO. . . . . . . . . . . . . . . . 50

3.8 Comparison of results of analysis neglecting source terms with analytic results ...................... 52

3.9 Comparison of results of analysis with data generated at MTX chord positions with analytic forms . . . . . . . . . . . . .

3.10 Comparison of results of analysis with data generated at evenly spaced positions with the analytic forms ............ 54

3.11 The function $\mathrm{G}(\mathrm{t})$ used in numerical simulation in the time domain. . $\quad 56$

3.12 Comparison of simulated signal with and without filtering. . . . . . 57

3.13 Transport coefficients obtained with filtering of simulated data. . . . 57 
4.1 Line-averaged densities and currents at which gas modulation data was taken. . . . . . . . . . . . . . . . . . . .

4.2 Weak sawteeth on central polychrometer channel, shot $14079 . \ldots 61$

4.3 Background density profile and fit to chord data for shot 14108. . . . 62

4.4 Change in FIR chord signals following a gas puff for $\bar{n}_{e}=3.3 \times 10^{20} \mathrm{~m}^{-3}$, $\mathrm{I}_{p}=285 \mathrm{kA} \ldots \ldots \ldots \ldots \ldots \ldots \ldots \ldots \ldots \ldots \ldots \ldots \ldots \ldots \ldots \ldots . \ldots \ldots$

4.5 Evolution of density profile vs. flux surface radius and time during a gas puff. . . . . . . . . . . . . . . .

4.6 Evolution of the density profile at several times steps following the gas puff, $\bar{n}_{e}=1.8, \mathrm{I}_{p}=285 \mathrm{kA} . \ldots \ldots \ldots \ldots \ldots$

4.7 Density profiles obtained from basis function fits to $285 \mathrm{kA}$ data at 280 msec. . . . . . . . . . . . . . . . . . .

4.8 Density profiles obtained from matrix inversion of $285 \mathrm{kA}$ data at 280 msec. . . . . . . . . . . . . . . . . . .

4.9 Marfe on visible bremstrahllung chord at $\mathrm{r}=-12.6 \mathrm{~cm}$ for shot 14038 with $\bar{n}_{e, 20}=2.9$ and $\mathrm{I}_{p}=285 \mathrm{kA} \ldots \ldots \ldots \ldots \ldots \ldots$

4.10 Black body temperature profiles for $285 \mathrm{kA}$ plasmas at $280 \mathrm{msec}$. Outside of $\mathrm{r} / \mathrm{a}=0.8$ the emission is not optically black. . . . . . . .

4.11 Particle transport coefficients overlaid from $285 \mathrm{kA}$ shots with $\mathrm{n}_{e, 20}=$ $1.8,2.2,2.6,2.9,3.3 \ldots \ldots \ldots \ldots \ldots \ldots \ldots \ldots$

4.12 Global energy confinement times measured by the diamagnetic loop. . 77

4.13 Values of $\mathrm{aV} / \mathrm{D}$ versus $\mathrm{a} / \mathrm{L}_{\mathrm{n}}$ for $0.4<\mathrm{r} / \mathrm{a}<0.8 \ldots \ldots \ldots$

4.14 Measured perturbed convection velocity versus Ware pinch. . . . . . 82

4.15 Background density fit for shot 13301 at 150 msec. . . . . . . . . 83

4.16 Amplitude and phases of $15 \mathrm{~Hz}$ components of the chords relative to the central chord for shot 13301... . . . . . . . . . . . 84

4.17 Fundamental $15 \mathrm{~Hz}$ component of modulation in shot 13301 at several time steps. . . . . . . . . . . . . . . . .

4.18 Relative amplitudes and phases of $15 \mathrm{~Hz}$ fundamental modulation component of shot $13527 \ldots \ldots \ldots \ldots$. . . . . . . . .

4.19 Comparison of transport coefficients obtained from spectral and time domain analysis of shots $13522-13527 . \ldots \ldots \ldots \ldots \ldots$

4.20 Representative uncertainties in diffusion coefficient values for plasma

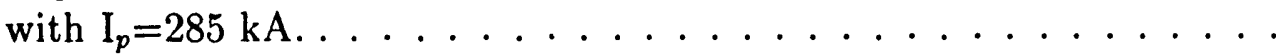

4.21 Central FIR chord signals during gas modulation at $\bar{n}_{e, 20}=3.3,2.9$.

4.22 Gas valve pressure transducer signals during modulation of $285 \mathrm{kA}$ shots 1393,14036 at $\bar{n}_{e, 20}=2.9,3.3$ respectively. . . . . . . . . .

5.1 Scaling of resistivity gradient driven turbulence diffusion coefficients for plasmas of the $285 \mathrm{kA}$ density scan. . . . . . . . . . . . .

5.2 RGDT diffusion coefficient values scaled, according to assumptions, from values in ref. $92 . \ldots \ldots \ldots \ldots \ldots$

5.3 Steady state power flux calculated by ONETWO. . . . . . . . . . 108

5.4 Perturbed resistive ballooning coefficients for plasmas of the $285 \mathrm{kA}$ density scan. . . . . . . . . . . . . . . . 115 
F.1 Plot of $|\mathrm{U}(\mathrm{f})|$ versus frequency . . . . . . . . . . . . 151

F.2 Fourier transform of Hanning function. . . . . . . . . . . . . 152

F.3 The results of analysis of data generated at MTX chord positions with complementary spectral analysis method. . . . . . . . . 156

G.1 Representative plots of residuals from fit versus other variables. . . . 159

G.2 Normal plots for fit to $\bar{n}_{e, 20}=2.6, \mathrm{I}_{p}=285 \mathrm{kA}$ data at $\rho=.55 . \ldots 160$

G.3 Comparison of empirical flux with linear fit. . . . . . . . . . . 162

H.1 Comparison of perturbed diffusion coefficients obtained with implicit or explicit consideration of $\mathrm{T}_{e}$ perturbations. . . . . . . . 171

H.2 Estimates of $\frac{\partial \Gamma}{\partial n}$ obtained from p. c. regression . . . . . . . . 172 


\section{Acknowledgements}

This research would not have been possible without the efforts of several of people. In particular I want to thank Bob Geer, Joe Burris and Reg Wood for their help in producing plasmas meeting the uncharacteristically stringent requirements entailed in this experiment and for operating the tokamak until late in the evening on several occasions. The high quality density profile data necessary for this research was obtained from the interferometer system develcped and maintained by Brad Rice and Tom Casper. It seemed Brad was always available to help make idjustments when difficulties arose. In addition he provided calculations of the equilibrium geometry obtained with use of polarimeter data.

I also thank Charles Lasnier for ECE data, Jim Foote for Thomson scattering measurements and Mike Makowski for magnetic loop data. Jeff Moeller assisted in the acquisition of signals needed for my work on the development of the diamagnetic loop. I thank Steve Allen for scheduling run time for these experiments.

Electrical technician Bob Geer always willing to help troubleshoot electronics problems and locate equipment during my time here. I also appreciate the help of mechanical technicians John Jolly and Dan Behne.

I thank Keith Thomassen for introducing me to the MTX experiment. Members of my dissertation committee, Keith Thomassen, Ed Morse and Allan Lichtenberg, deserve credit for responding to my work in a timely manner and for contributing several helpful suggestions.

I am especially grateful to Bick Hooper for many stimulating discussions. During the time I have worked on this and other projects with the MTX experiment, Bick has inspired me by continuously exemplifying the highest standards in his knowledge of experimental plasma physics while remaining genuinely committed to helping me progress. He was always available to listen to my ideas, was keenly interested in looking at data and was supportive as I pursued the goals of these experiments.

The MTX group succeeded in surmounting many difficult technical and financial obstacles - until the self-destruction of the interferometer laser one evening. Without such dedication this research might not have been carried out. 


\section{Chapter 1}

\section{Introduction and Background}

\subsection{Density Limits}

A prerequisite for achieving ignition in future magnetic fusion plasma experiments is the production of stable plasmas with central densities near $(2-5) \times 10^{20} \mathrm{~m}^{-3}$. In the design for ITER the average densities for these ignited plasmas are expected to be near or beyond the limits according to the empirical Greenwald scaling. Tokamak plasmas at the density limit often terminate by disruptions which could have an adverse impact on the reliability of operations in this machine. Thus it is desirable to enhance our understanding of the mechanisms responsible for this limit.

Limits to the maximum density that can be attained in tokamaks are often classified into one of two types. One type, known as the Murakami limit, has been parameterized by $\bar{n}_{e} \leq \mathrm{MB}_{t} / \mathrm{R}$. The Murakami number $\mathrm{M}$ is increased as additional heating is applied [1, 2] or as $Z_{\text {eff }}$ approaches one [37]. This density limit has been attributed to a loss of balance between input power and radiated power [38, 9]. The disruption is preceded by an increase in the radiated power which leads to a locally unfavorable power balance [10]. It has been observed that much higher values of the Murakami parameter can be obtained at low values of $\mathrm{B}_{t}$ than at high $\mathrm{B}_{t}$, so this limit may not scale as $\mathrm{B}_{t} / \mathrm{R}$ [11].

A second type of density limit is apparent when $\bar{n}_{e}$ is plotted for a large number of shots versus plasma current. The earliest plots of this kind, known as Hugill plots, 
display the Murakami number versus 1/q. The pattern which emerges is that the tokamak operating space appears to be constrained to a region with a boundary having a slope proportional to 1/q. Based on data from Alcator C, DIII-D and PBX, Greenwald derived the following scaling expression

$$
\bar{n}=\kappa \bar{J}
$$

where $\bar{n}$ is the central line-averaged density measured in units of $10^{20} \mathrm{~m}^{-3}, \kappa$ is the elongation parameter and $\bar{J}$ is the average current density in MA-m ${ }^{-2}[16]$. This is not an exact scaling and it is not obeyed by all machines. But there is an ample amount of empirical evidence that in ohmic discharges (where $q$ is not too low) a Greenwaldtype scaling holds with $\bar{n}_{e}=C \kappa \bar{J}$. The constant $\mathrm{C}$ varies somewhat from device to device and generally increases with the purity of the discharge [13]. The density limit in a reversed field pinch and for ultra low-q $\left(\mathrm{q}_{a}<1\right)$ devices can be described by a similar scaling parameter $\mathrm{nRq} / \mathrm{B}_{\boldsymbol{t}}$. In an RFP, the density limit is called the "lower I/N" limit, with I being the plasma current and $\mathrm{N}$ the line density [12]. This limit can be the same as the Greenwald type limit found in tokamaks [10]. These results imply there could be a single cause for the variety of behaviors that are observed near the density limit.

With the addition of auxilliary power in the form of neutral beams or $r$. $f$. the density limit at fixed current can be increased. Present experimental data have been represented by the power law scaling

$$
\bar{n}_{e}=C \kappa J\left(\frac{P_{i n}}{P_{\Omega}}\right)^{\alpha}
$$

The exponent $\alpha$ varies between the different machines and can even vary in different operating configurations on the same device. Values of $\alpha$ as large as 0.5 have been observed in JT-60 and in the old divertor configuration of ASDEX. In the newer [14] ASDEX divertor configuration a rather weaker dependence on power was observed with $\alpha \leq 0.25$. A comparatively weak dependence with neutral beam power is observed on DIII-D, with $0 \leq \alpha \leq 0.2$. 


\subsection{Fuelling Limits}

In the larger machines, where the ratio of the mean free path for low energy neutrals to the minor radius is less than for the smaller devices, substantial differences are seen in the operating ranges attainable with gas fuelling and with pellets. By injecting high speed pellets of frozen fuel into the plasma core, the difficulties associated with gas puffing can be avoided. Fuel may be introduced into the plasma core with neutral beams yielding similar results.

The maximum obtainable density can be influenced by the limiter/divertor employed. For example the highest densities obtainable in machines with carbon limiters and gas fuelling are approximately half of those with molybdenum or beryllium limiters [16]. With pellet fuelling on Alcator $\mathrm{C}$ with carbon limiters the same density limit is obtained as with gas fuelling and molybdenum. The variation in the limits can be caused by the differences in the pumping effects of the limiters and divertors as well as changes in impurity levels they introduce.

\subsection{Density Limit Physics and Phenomena}

The density limit is most often manifested as a termination of the discharge by a major disruption. Because the plasma electron density does not appear explicitly in the equations of magnetohydrodynamics, it would appear that the loss of MHD stability is merely the final manifestation resulting from some other underlying cause.

A widely accepted notion is that cooling of the plasma edge causes the current profile to contract, resulting in an increase in the current density gradient near the $\mathrm{q}=2$ surface and causing the $\mathrm{m} / \mathrm{n}=2 / 1$ mode to become unstable [13]. A number of mechanisms can cause edge cooling. Impurity radiation, charge exchange, ionization losses or a deterioration of energy or particle confinement in the plasma edge can all be important. If the edge particle confinement deteriorates with respect to energy confinement then the ratio of energy flux to particle flux decreases, causing a decrease in the average particle energy. Increased particle transport can also effect the edge 
density and impact particle recycling.

Early theories focused on edge radiation as the cause behind the density limit disruption $[38,9]$. Radiation losses increase with density while input power does not, making this a plausible mechanism for plasma edge cooling. A number of authors have derived scalings for the density limit based upon thermal instability and MHD collapse caused by impurities radiating in thin shells at the plasma boundary. An example is the following scaling [15]

$$
n_{\max } \sim \frac{B}{q R}\left(\frac{Z_{e f f}}{Z_{e f f}-1}\right)^{\frac{1}{2}} \frac{1}{\bar{T}_{e}^{\frac{1}{2}}}
$$

which gives a maximum edge density that increases as $\mathrm{Z}_{\text {eff }}$ is reduced. Results from several experiments indicate that the density limit depends strongly upon the edge density. On JET [3], ASDEX [34] and JT-60 [10] higher central and line-averaged densities can be achieved with pellet injection than with gas fuelling. The maximum edge densities are, however, quite similar for the pellet and gas fuelled discharges. The improvement in the maximum line-averaged density with pellet fuelling is a profile effect.

The density limit for a series of gas fuelled and pellet fuelled limiter discharges was studied in JT-60 [10]. These were high current/low q discharges, with $\mathrm{q}_{c y l}=2.1$ -2.4 , and were thus near the region of the Hugill plot generally associated with the Murakami limit. In the early stages of the disruption sequence, radiated power from the plasma edge approaches $100 \%$ of the input power. The line-averaged density in the outer portion of the plasma was measured with the FIR interferometer viewing along a chord $50 \mathrm{~cm}$ off axis from the center of the vessel $(\mathrm{a}=90 \mathrm{~cm})$. A plot of $\bar{n}_{e}$ $(\mathrm{r}=50 \mathrm{~cm}) \mathrm{Rq}_{\mathrm{cyl}} / \mathrm{B}_{\imath}$ versus absolute input power shows an upper limit to the region of stable operation which scales approximately as $\mathrm{P}_{a b s}^{0.5}$. Below this region most of the plasmas are stable, but disruptive discharges are scattered well into the region of lower density. The data indicates that disruptive shots tend to have a higher ratio of power radiated in the exterior region to absolute input power. The density limit rises with increasing $\mathrm{P}_{a b s}$ and decreasing $\mathrm{Z}_{\text {eff }}$.

The electron temperature must be considered significant in determining the effect 
of radiated power on the power balance. For carbon and oxygen impurities, the dominant light impurity species in JT-60, the radiated power at coronal equilibrium increases rapidly with decreasing electron temperature for $T_{e}<0.3-0.5 \mathrm{keV}$ and especially fast for $T_{e}<100 \mathrm{ev}$. The electron temperature at $\mathrm{r}=80 \mathrm{~cm}$ is $<0.5 \mathrm{keV}$ at the time the disruptions are triggered in disruption shots studied. This value is systematically lower than values for stable discharges having the same values of $\mathrm{P}_{a b s}$ and $\left.\bar{n}_{e}^{2} \overline{Z_{\text {eff }}}\right|_{r=50 \mathrm{~cm}}$. These observations for these high current shots considered, are consistent with the picture of a radiative instability causing a loss of equilibrium in the power balance in the outer portion of the plasma. In particular, models of the density limit based upon radiative power balance in the plasma edge "predict" that the limit to the edge density should scale approximately as $\mathrm{P}_{a b s}^{0.5}$.

Data from JET also shows an upper limit to the edge density that can be obtained which scales as $\mathrm{P}_{a b s}^{0.5}$. Studies of the density limit in JET with carbon limiters [20, 3] found that this limit was always disruptive. As the density was increased the radiated power eventually exceeded the input power, resulting in a thermal instability in the plasma edge. As a result the electron temperature profile contracted. The associated narrowing of the current density profile destabilized a sequence of MHD modes, particularly the $\mathrm{m} / \mathrm{n}=2 / 1$ mode, which grew, locked and caused a major disruption. When evaporated beryllium layers and beryllium limiters were used the density limits were increased by roughly $50 \%$ compared to those with carbon limiters. At moderate densities $\left(\bar{n}_{e} \sim 3 \times 10^{19} \mathrm{~m}^{-3}\right)$ the steady state level of radiation could be only $50 \%$ of that for equivalent discharges bounded by carbon.

There are problems with the density limit model based on radiated power balance. The global database for the operating region of JT-60 shows an upper limit of $\bar{n}_{e}(\mathrm{r}=50$ cm) $\mathrm{Rq}_{c y l} / \mathrm{B}_{t}$ which scales with input power as $\mathrm{P}_{a b s}^{0.5}$. For a fixed total input power, the upper limit of $\bar{n}_{e}(\mathrm{r}=50 \mathrm{~cm}) \mathrm{Rq}_{c y l} / \mathrm{B}_{t}$ appears to be constant over a wide range of $q_{c y l}$. The persistence of this Hugill-type limitation cannot be explained by models based upon the radiated power balance, particularly for cases where $\mathrm{P}_{N B}>>\mathrm{P}_{O H}$. The variable $\bar{n} R q / B_{t}$ can be considered as dimensionless and scales as the square root of the ratio of radiated power to ohmic input power [36]. The notion that the Hugill 
scaling of the density limit for ohmic plasmas results because this parameter directly relates to the power balance does not explain the fact the Hugill-type scaling persists even for high power neutral beam heated discharges in JT-60.

In Alcator $\mathrm{C}$ the Greenwald density limit is accessible for values of $\mathrm{Z}_{\text {eff }}$ up to 1.5 and perhaps even as large as 2 . Theoretical scalings based on a radiated power balance, such as eq. 1.3, predict that the limit density should diverge as $Z_{\text {eff }}$ approaches 1. In the outer regions of the plasma, impurity radiation dominates over hydrogen bremsstrahlung for $Z_{\text {eff }}$ only slightly larger than 1 . This suggests that some alternate rnechanism plays a dominant role in determining the density limit in Alcator C.

The behavior of the particle confinement near the density limit in Alcator $\mathrm{C}$ was examined in a series of pellet injection experiments [17]. The results are described by the following passage from reference [16].

"In these experiments, single pellets were injected into plasmas with relatively low plasma current. The density increased very quickly at the time of injection $(0.27 \mathrm{sec})$ for all discharges and the rate of density decay was monitored. As the plasma current was lowered from shot to shot, the decay time decreased dramatically. ... the density limit, established with gas fuelling, was greatly exceeded. These discharges did not disrupt, however, but simply 'shed' particles in excess of the limit. The density decay time is not the same as the particle confinement time but is closely related. At steady state the particle confinement time is given by the ratio of density to source; however, in those cases where the time derivative dominates the source term, the density decay time will equal the particle confinement time $\tau_{p}$. Unlike the conventionally defined global $\tau_{p}$, which is dominated by the large particle fluxes in the plasma edge, this confinement time is characteristic of the plasma core. It is worth pointing out that no decline in energy confinement accompanied the drop in particle confinement except for the convective loss directly associated with the density decay."

As shown in Fig. 1.1, the rapid drop in particle confinement occurs for values of $\bar{J} / \bar{n}$ 


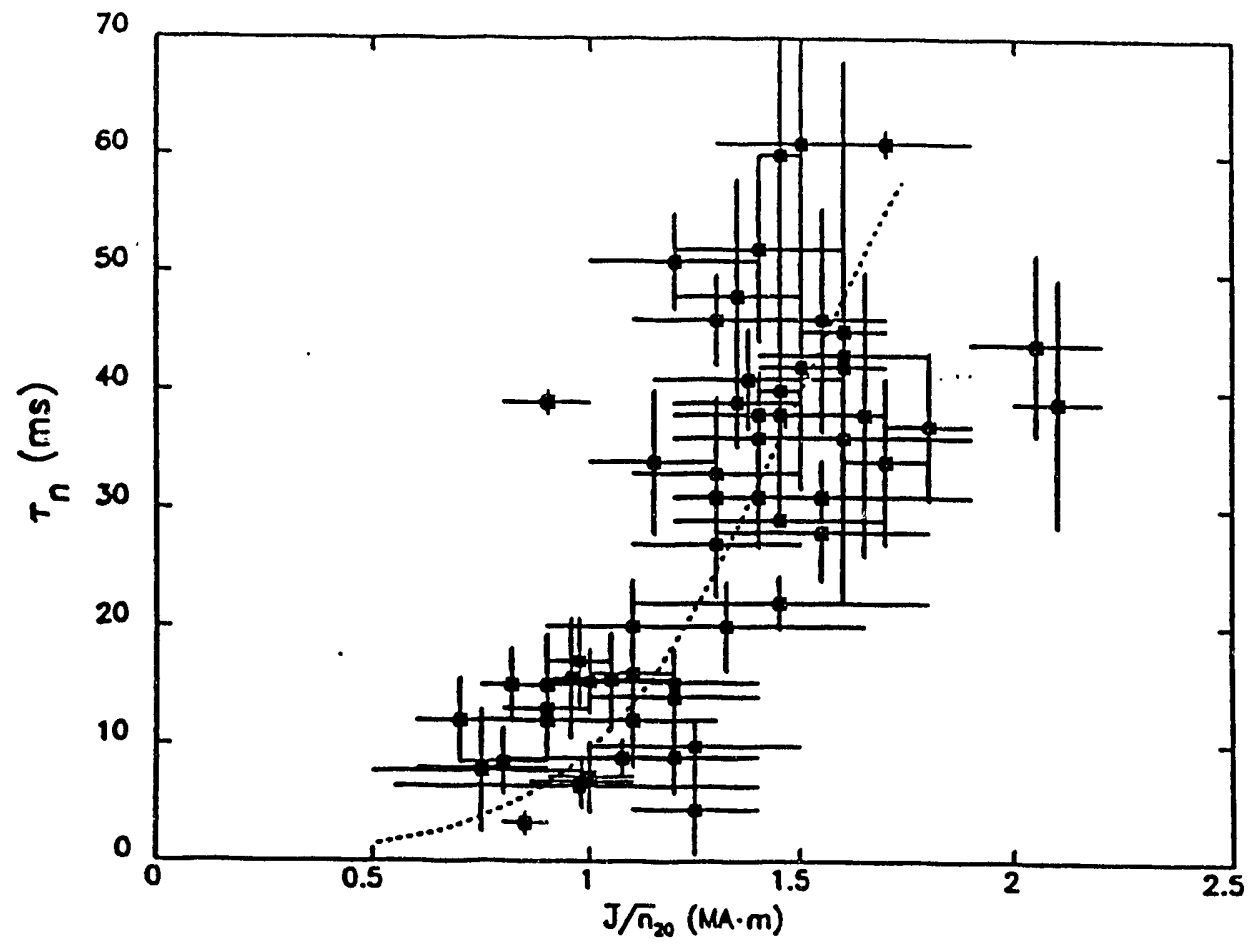

Figure 1.1: Density decay time (after pellet injection) versus $\bar{J} / \bar{n}$. (from Greenwald et al. [16])

near the previously defined Greenwald limit. The dramatic drop in density decay time is symptomatic of a dramatic deterioration of particle confinement in the plasma core.

"Marfes" are bands of low temperature poloidally asymmetric plasma which appear in most tokamaks as the density limit is approached. They are always localized to the inside of the torus near the limiter and are characterized by large amplitude density fluctuations and large amounts of radiated power. They are thought to be a thermal condensation phenomenon $[18,19]$ localized to the inside of the torus by neoclassical flows. Greenwald et al. [16] postulated that Marfes may by early symptoms of deteriorating particle confinement. In this picture, as particle confinement deteriorates relative to energy confinement, the average energy of edge particles decreases, 
setting up the right conditions for a radiative condensation.

In JET once the radiated power was reduced compared to the level for an equivalent carbon bounded discharge through the introduction of beryllium there was a fundamental change in the plasma behavior near the density limit $[3,20]$. As the density was increased to near the limiting density, the radiated power still increased rapidly. Following this, however, a Marfe formed on the inner wall of the tokamak, resulting in an increase in radiated power which could exceed input power for a time. In JET Marfes occur when $\mathrm{T}_{e}$ at the limiter falls to 10 to $20 \mathrm{ev}$. When the Marfe forms the global particle confinement time falls along with the edge plasma density. This decrease in edge density is most likely caused either by screening of neutrals, a reduction of power flow to the scrape off layer [136], changes in recycling (the limiter interaction is much reduced), or a deterioration of particle confinement in the edge plasma [20]. The result is that the Marfe terminates. The entire cycle can re-occur if fuelling continues. Since there is little MHD activity during these events density limit disruptions are quite rare in JET plasmas with beryllium limiters.

With Beryllium limiters the density limit in both ohmic and auxilliary heated JET plasmas has been extended substantially beyond that obtained with graphite limiters as a result of reduced impurity radiation. Besides impurity levels, the density and temperature of the edge plasma appear to be quite important in determining the limit density in JET, since this limit is extended by auxilliary heating and deep pellet fuelling. In JET discharges with beryllium limiters the density limit appears to be more a refuelling than a radiative limit. Radiation and particle confinement may both play a role.

Density limit discharges were studied on the DITE tokamak at high values of edge $q=4-8$ to minimize the occurrence of disruptions [19]. As the density was raised there was a gradual narrowing of the plasma temperature and current profiles. A reduction in the edge temperature is measured and Marfes may appear. For $q \geq 6$ continued contraction of the profiles leads to formation of a detached plasma which remains stable. A detached plasma is characterized by an inward contraction of the temperature profile from the limiter. The discharge is surrounded by a symmetric band of low 
temperature plasma which radiates essentially $100 \%$ of the input power. The profile contraction, observed as the density is raised, is the result of a deterioration of edge confinement relative to the center and may be explained only partly by radiation losses [19]. A large fraction of the input power must be transported to the edge of the plasma to sustain the radiation losses observed in the Marfes. Application of neutral beam heating does not result in a significant rise in edge temperature, but instead causes an increase in radiation from the Marfe. The limit density is increased. Allen et al. state [19]:

"The Marfe can be avoided by adjusting the plasma position in the limiter aperture but this does not affect the density limit appreciably. It appears to be a symptom of low edge temperature rather than the cause of it."

Particle transport in the plasma edge plays a dominant role in the density limit behavior of the TUMAN-3 tokamak [21]. In experiments the density was increased with gas puffing while the current was ramped up concurrently with magnetic compression of the minor radius of the plasma. The plasma density increased in a "steplike" fashion. Periods where the slope of the density increase was reduced coincided with pronounced increases in the $\mathrm{H}_{\alpha}$ signal, implying steplike changes in the global particle confinement time. A high density mode (HDM), characterized by increased values of the density limit and enhanced energy confinement, is achieved when the instability responsible for increased edge recycling is suppressed. In experiments when the current alone was quickly ramped up, an increase in the $\mathrm{H}_{\alpha}$ emission symptomatic of an increase in recycling was observed [22]. In this case the density could not be increased.

The density limit in T-10 is characterized by a well defined sequence of events [33]. First, as $\bar{n}_{e}$ is increased, confinement deteriorates in at least the plasma exterior, resulting in an enhanced plasma-wall interaction. This coincides with decreasing electron temperature in the plasma edge. A plasma cooling front moves inward. An increase in edge density fluctuations during gas puffing is measured with a reflectometer and it seems to be associated with edge cooling [22]. In the second stage the $\mathrm{D}_{\alpha}$ line intensity decreases along with a decrease in the plasma density in the exterior 
portion of the plasma. The third stage is punctuated by a burst of MHD activity during which "partial disruptions" cause the plasma to lose a substantial amount of energy. The fourth and final stage is a major disruption, resulting in termination of the discharge. A sharp decrease in the decay time of pellet fuelled T-10 discharges is reported at densities near the limiting density [39]. In TEXT a transient enhancement of density fluctuation levels associated with microturbulence is observed to precede density limit disruptions [40].

Thus there is substantial evidence that particle confinement plays a dominant role in the density limit dynamics on at least several tokamaks. When particle confinement deteriorates at the edge a greater fuelling source is required for an incremental increase in density. Recycling is increased, resulting in cooling of the edge plasma. If the edge density is increased greater radiation will result in further cooling. Radiation and a deterioration in particle or energy confinement can act to cool the plasma edge simultineously. The relative importance of each varies between different machines and even between different regimes of operation in the same machine. If the plasma is sufficiently dircy, the increase in radiation will cause the operating boundaries to contract. If particle confinement is the dominant mechanism then one would not expect a reduction in impurity content of the plasma to result in an increase in the density limit. This evinces comparison with the density limit scaling observed for relatively clean Alcator $\mathrm{C}$ plasmas.

It is possible that the two mechanisms are closely associated. For example radiation is considered a viable driving term in several current theories on edge turbulence [41, 42]. Experimental evidence from TEXT supports the notion that radiation is an important driver of edge turbulence [43, 4]. If turbulent transport is enhanced in the edge plasma by an increase in radiation it could cause the plasma to cool even more than the radiation alone, playing an important role in the instability. 


\subsection{Summary of Experiment and Results}

Because of the importance of high density operation in future tokamak experiments, an enhancement in our understanding of the transport process responsible for the degradation of particle confinement which has been observed near the density limit in several machines would be valuable. The MTX experiment is aptly suited to contribute in this regard. Its principal components, shown in Fig. 1.2, are the Alcator $\mathrm{C}$ tokamak and a free electron laser used to produce high power microwaves for use in electron cyclotron resonance heating experiments. This laser was not used in the experiments considered here. The Alcator $\mathrm{C}$ tokamak has a high toroidal field strength (3-13 T) and produces high density plasmas $\left(\bar{n}_{e}\right.$ up to $\left.10^{21} \mathrm{~m}^{-3}\right)$. The ranges of both of these parameters are relevant to tokamak experiments planned for the future. In addition, when operated at fairly high $\mathrm{q}_{a}$ the Alcator $\mathrm{C}$ plasma generally remains well behaved near the density limit. The standard $16.5 \mathrm{~cm}$ limiters were used so that the machine was in a configuration essentially identical to that used during operations at MIT.

The primary focus of this work is a series of experiments aimed at increasing our understanding of the transport processes responsible for the deterioration in particle confinement in the Alcator $\mathrm{C}$ core plasma near the density limit. A gas modulation technique was used to modify the density profile, which was measured with good resolution using a 15 chord far-infrared interferometer. Small changes in the density profiles were created in such a way that perturbed particle convection and diffusion coefficients could be extracted using linear perturbation analysis. Local temperature perturbations were also produced which were highly correlated with the density perturbations, but of opposite sign. The effect of these temperature perturbations is manifest in the perturbed convection velocity, $\mathrm{V}$, as described in chapter 2.

A key result of this study is the variation of particle transport coefficients as the density limit was approached in a series of shots at a constant current of $285 \mathrm{kA}$ shown in fig. 1.3. Diffusion coefficients showed little variation with density in the saturated ohmic confinement regime between $2.2 \times 10^{20} \leq \bar{n}_{e} \leq 2.9 \times 10^{20}$. At the 


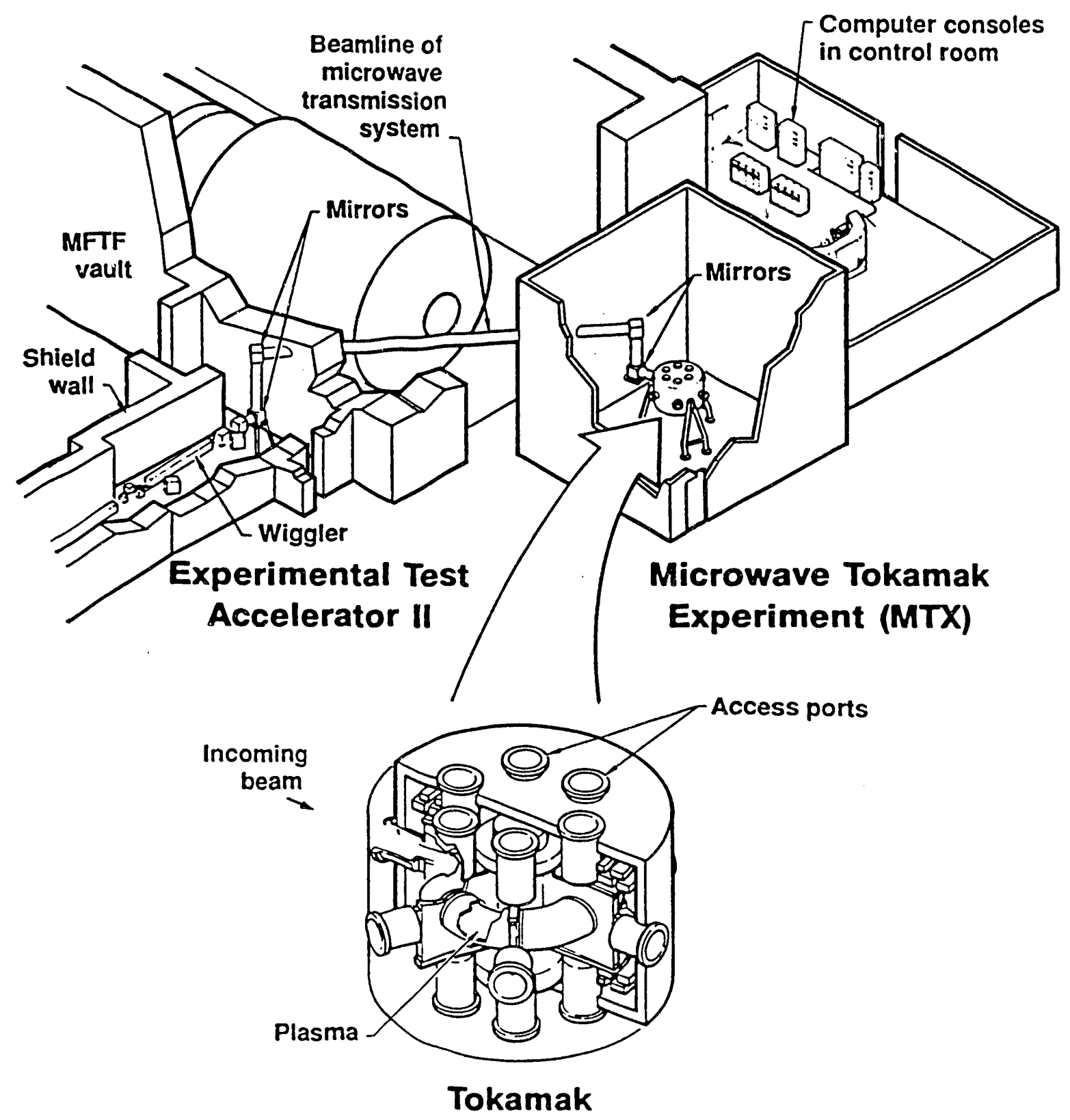

Figure 1.2: Overview of the Microwave Tokamak Experiment 

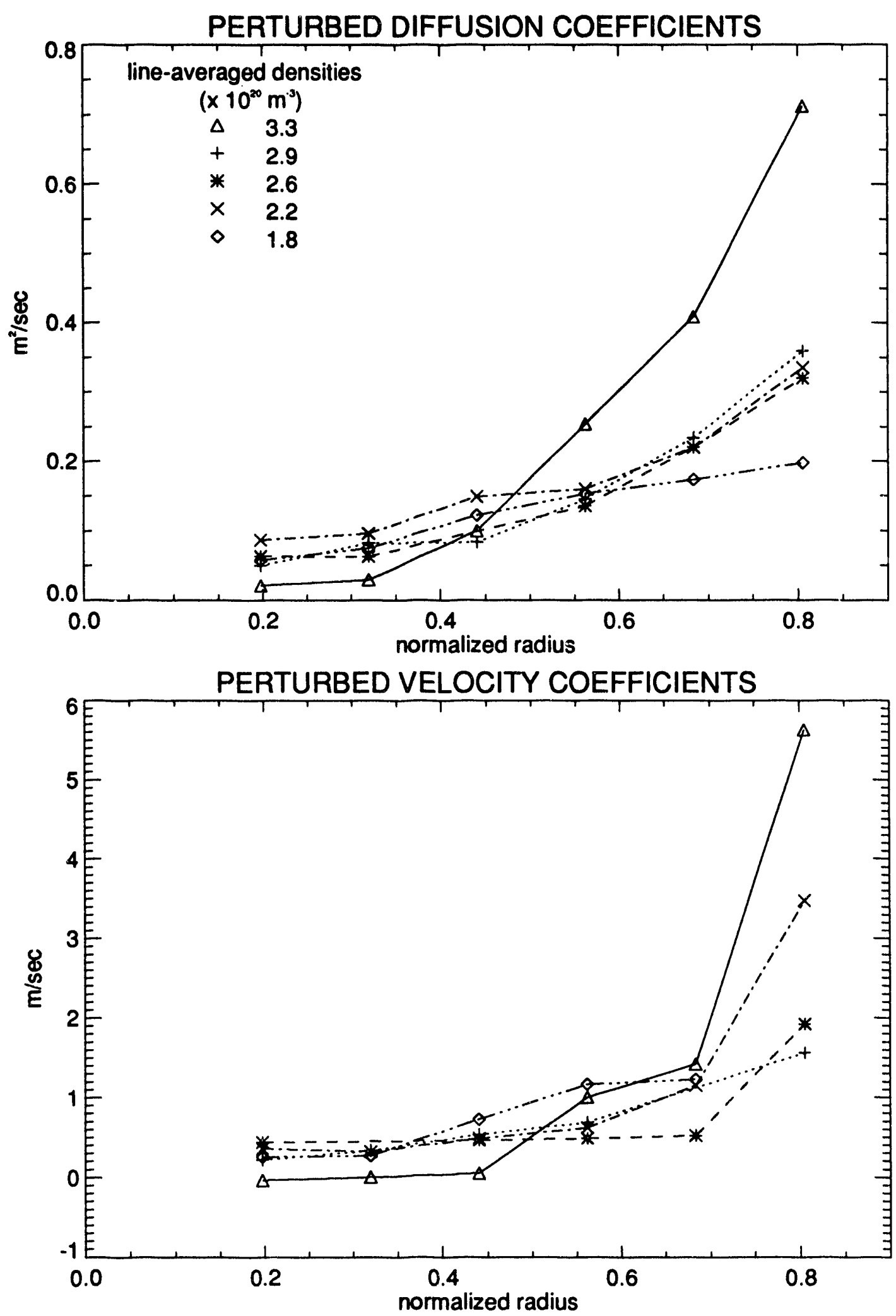

Figure 1.3: Particle transport coefficients overlaid from $285 \mathrm{kA}$ shots with $\mathrm{n}_{e, 20}=1.8$, $2.2,2.6,2.9,3.3$. 


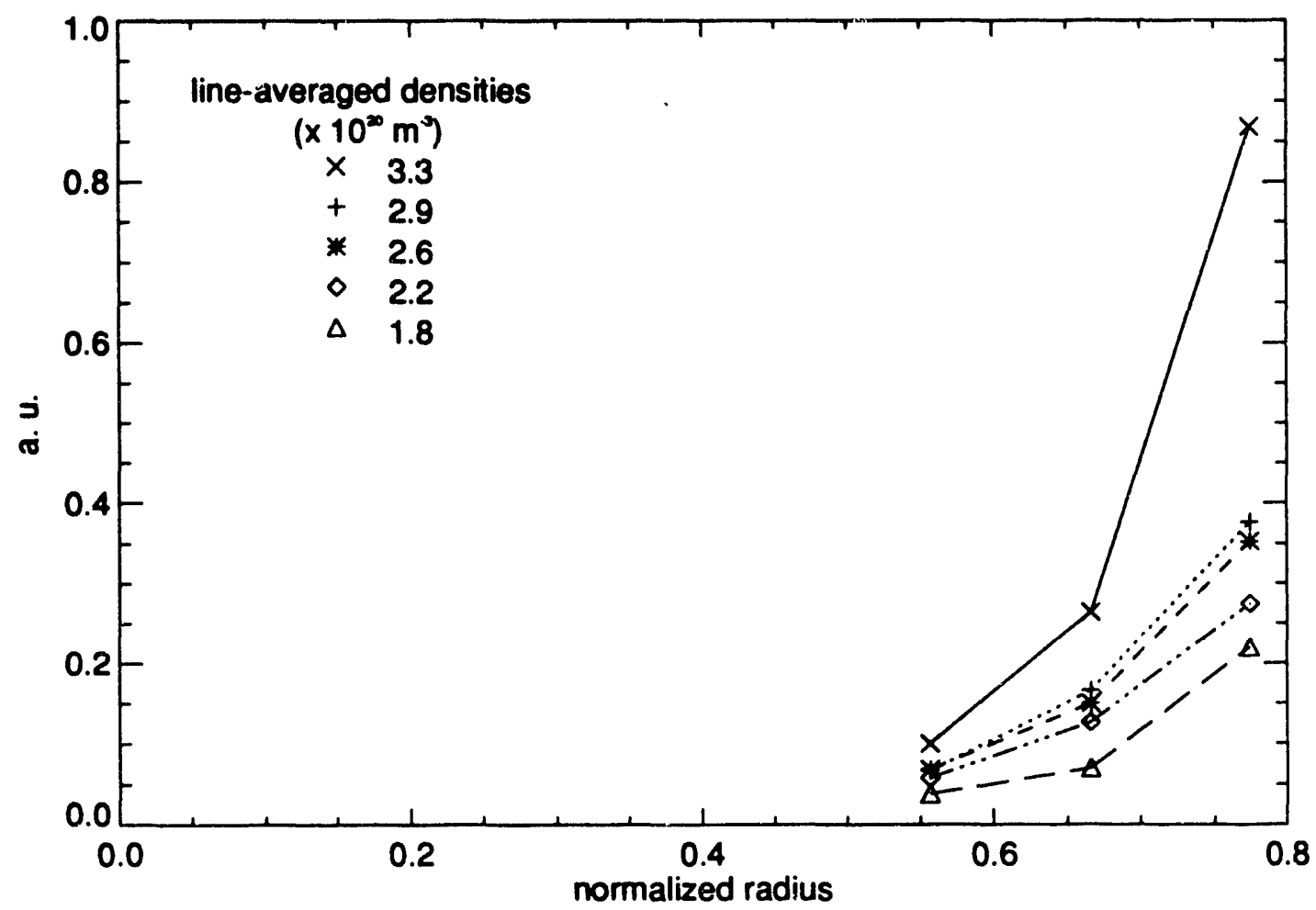

Figure 1.4: Scaling of resistivity gradient driven turbulence diffusion coefficients for plasmas of the $285 \mathrm{kA}$ density scan.

Greenwald density limit threshold, which occured at $\bar{n}_{e}=3.3 \times 10^{20}$ for this plasma current, a dramatic increase was seen in both the perturbed convective and diffusive transport coefficients in the outer region of the plasma. Density profiles maintained a similar shape near the density limit, congruous with the similar behavior of the transport coefficients.

Despite the large rise in the particle transport coefficients no dramatic deterioration was evident in the global energy confinement time. Plasmas in the region where increases in the transport coefficients were measured $(0.6 \leq \mathrm{r} / \mathrm{a} \leq 0.8)$ had relatively low temperatures $\left(\mathrm{T}_{e}<250 \mathrm{ev}\right)$ and were in the Pfirsch-Schlüter collisionality regime. The scaling of $\mathrm{D}$ in this region of these high density plasmas is seen to compare well with the scaling given in a nonlinear theory of resistivity-gradient-driven turbulence, which is evaluated for the plasma profiles in fig. 1.4. Inverse dependences on the temperature and temperature gradients were key components of the model most closely associated with this outcome. 
This result, taken with those from other machines as discussed in chapter 6, suggests that the enhanced transport which has been observed in the outer regions of the bulk plasma on several tokamaks near the density limits is precipitated by narrowing of the temperature profile. The coefficient profiles provide evidence that the mechanism responsible is localized to regions of plasma having relatively low temperatures and causes particle transport coefficients to increase through an inverse dependence on $\mathrm{T}_{e}$ and/or $\mathrm{L}_{T e}$ characteristic of several resistive edge turbulence theories.

\subsection{Outline of Thesis}

The remaining chapters of this thesis are organized as follows. Guided by consideration of theory as well as characteristics of particle transport observed in experiments, a framework for the description of particle transport is outlined in chapter 2. The consequences of the transport coefficient dependences on plasma parameters are examined. Then, following a description of the gas modulation technique used in this study, several considerations relating to the experimental method are covered.

The method employed for analysis of modulation data is described in chapter 3 . A procedure for obtaining a basis function fit for the density profile from interferometer measurements is derived. The routine incorporates a self-consistent correction for refraction of the interferometer beam. It is shown that the correction for refraction effects is stationary for small changes to the density profile. Since the plasma position can also be treated as constant, analysis routines based upon linear equations are developed for use in determining the small changes in the density profile during modulation. Separate versions are introduced which utilize spectral and linear regression methods to determine the perturbed particle transport coefficients. The influence of spline fitting performed by the routines as well as the particle source term and filtering of the interferometer signals are considered.

Experimental results are discussed in chapter 4. The behavior of the density, electron temperature and particle transport coefficient profiles as well as the global energy confinement time near the density limit are described. The chapter also in- 
cludes observations about particle transport not related to the density limit. The quality of the model's fit to the data is discussed. The significance of temperature perturbations is considered. Estimates are made of the uncertainties in empirical values of transport coefficients.

The measured particle transport coefficients are compared with predictions of various theories of plasma turbulence in chapter 5 . The role of certain plasma variables is elucidated. In chapter 6 similarities between the characteristics of the particle confinement deterioration on various machines are examined and interpreted in light of the results of this study.

Chapter 7 restates the major findings of this thesis. 


\section{Chapter 2}

\section{Method of Experiment and Theory of Data Analysis}

In fusion research we are interested only in plasmas which attain gross stability by satisfying a macroscopic force balance. Once a plasma attains gross stability we can consider the processes which govern the flow of energy and mass through the system, known as transport processes. Since fusion plasmas must be hot and confined, and are therefore constantly driven, resulting gradients of temperature, particle density and current density are sources of free energy. These sources can drive "microinstabilities", instabilities which are of a size scale of order the Larmor radius. These instabilities can evolve into a state of turbulence and can drive fluxes which tend to carry the system toward thermal equilibrium. Although fusion plasmas never achieve thermodynamic equilibrium they can reach a steady state, in which plasma parameters are constant in time, when a balance is obtained between the input and transport processes. The plasma is characterized as being close to thermodynamic equilibrium because it can be parameterized by intensive thermodynamic variables.

An appropriate framework for describing particle transport in a plasma will be outlined in the material which follows. Small changes in local plasma parameters created with a gas modulation technique can be related to changes in the local particle flux to obtain particle transport coefficients. The consequences of transport coefficient dependences on local plasma parameters will be examined. The experi- 
mental gas modulation method utilized for this study will be described along with key experimental considerations.

\subsection{Particle Transport}

In a tokamak geometry transport due to collisions is known as neoclassical transport. Neoclassical fluxes are described by elements of a transport matrix and are linearly proportional to gradients of plasma parameters. Though this formulation is useful for describing some fluxes under some circumstances, it is most often inadequate, particularly for describing particle and electron thermal transport in tokamaks. Yet the transport matrix has survived as a standardized framework in which to characterize plasma behavior. There can be cases when even this framework is not helpful. For example if the transport is determined by a turbulent flux which is an integral over the spectrum of fluctuating quantities with implicit dependences on plasma parameters [5]

$$
\Gamma=\int d \omega n \tilde{(\omega)} \phi(\omega)|\gamma(\omega)| k_{\theta}(\omega) \sin \alpha(\omega) / B_{t} \approx \kappa \beta\left(\frac{\tilde{n}}{n}\right)^{2} n^{\beta_{1}}
$$

it may be impossible to separate the flux into an unique representation with diagonal and off-diagonal terms. Even though this complication can affect the analysis of the steady state transport, it is not a problem for analysis of a perturbed flux using linear perturbation theory. Any analytic, differentiable expression for a flux can be expanded about an equilibrium value. When the steady state portion of the flux is subtracted off and only the terms linear in the perturbation are retained (appropriate for a small perturbation) the resulting expression for the perturbed flux can be written in the form of a transport matrix.

In the core of steady state tokamak plasmas there often exist substantial density gradients in regions which have no significant particle flux. Due, in part, to this observation it is customary to represent the radial particle flux as the sum of two components, a diffusive term and a counter balancing inward convection term:

$$
\Gamma_{r}=-\mathbf{D} \frac{\partial n}{\partial r}-\mathbf{V} n
$$


The particle flux is governed by the continuity equation,

$$
\frac{\partial n}{\partial t}+\nabla \cdot \Gamma=S
$$

where $\mathrm{S}$ is the particle source term. The divergence relation is applied to yield an expression for the radial flux at a flux surface:

$$
\int \nabla \cdot \Gamma d V=\oint \Gamma_{r} \cdot d S=\int\left(S-\frac{\partial n}{\partial t}\right) d V .
$$

In the case of circular flux surfaces this simplifies to

$$
\Gamma_{r}(r)=\frac{1}{r} \int_{0}^{r}\left(S\left(r^{\prime}\right)-\frac{\partial n\left(r^{\prime}\right)}{\partial t}\right) \mathbf{r}^{\prime} \mathrm{dr}^{\prime} .
$$

In gas fuelled tokamak plasmas the particle source term is usually localized to the edge because the neutral particle density in the plasma core is typically very small. By combining equations 2.2 and 2.5 one obtains an expression which is useful for an empirical description of particle transport.

\subsubsection{Previous Observations}

Two general approaches have been employed in the study of particle transport: the analysis of the steady state flux and the response of the flux to a perturbation in the density profile. In the former case it is impossible to separate the contributions from the two components of equation 2.2. In the latter case it is possible to create a significant flux over the entire plasma column from which the convective and diffusive components can be extracted.

In several studies $[6,7,8]$ particle transport was modelled during transient, large fractional amplitude changes in the density. These were typically created by gas puffing or resulted from the density decay following pellet injection. Reasonably good agreement between simulated and measured results were obtained using the above representation for the flux with assumed functional forms for the radial profiles of the transport coefficients. In order to fit the data credibly the transport coefficients in all of these cases $[6,7,8]$ must be much larger than the neoclassical values in at least the outer portions of the plasma column and generally in the interior region as 
well. Thus an anomalous inward pinch balances anomalous diffusion in at least the outer portion of those plasmas.

When perturbations to the density profile are fractionally small the transport coefficients may be obtained through linear perturbation analysis and may be associated with a specific local plasma state (i.e. local intensive variables). Perturbative density modulation techniques were first applied to the study of tokamak particle transport in T-10 by Bagdasarov et al. [48] and were extended through the applications to TEXT and ASDEX by Gentle [23, 80] and TFTR by Efthimion [24]. On TEXT periodic density perturbations were created with gas puffing. The local ratios of the perturbed to equilibrium fluxes were determined on TEXT for various amplitudes of the fractional central density modulation $\Delta n / n_{o}$ [25]. Published results indicate fractional changes in the local fluxes of order unity or larger were associated with small fractional density changes of around $13 \%$ or less. Modulation of the turbulence level was fractionally small as well. Also the profiles of the perturbed fluxes were linearly proportional to the density perturbation amplitude. Gentle et al. [25] considered the implications for single term factorable models for the flux in terms of local plasma parameters or local turbulence levels e.g.

$$
\Gamma=C n^{\alpha}(\nabla n)^{\beta} T^{\gamma}(\nabla T)^{\delta} \ldots
$$

or

$$
\Gamma=C n^{\alpha}\left(\frac{\tilde{n}}{n}\right)^{\beta} \ldots
$$

The linear terms in the expansion of eq. 2.6 scale as $\alpha\left(\Delta n / n_{o}\right)+\ldots \sim \Delta \Gamma / \Gamma_{o}$. They argue that since the perturbations to all of the local quantities, including turbulence levels, are of order $10 \%$ at least some of the exponents $\alpha, \beta$ and $\delta$ would have to be rather large in order for a single term of the form of eq. 2.6 to produce large fractional changes in the flux. Very strong dependences upon parameters $(\alpha>10)$ would be inconsistent with the observed linearity between the perturbed particle flux and density perturbation amplitudes. In addition the higher harmonic content of the flux would become more pronounced as the density wave propagated inward and there would be large differences between equilibrium and perturbed behavior, neither 
of which are observed. Gentle et al. thus showed that the particle $f l u x$ cannot be expressed as a single term factorable model, such as in equations 2.6 and 2.7. They point out that expressions which scale proportional to $\mathrm{a}+\mathrm{b} \eta_{e}, \mathrm{a}, \mathrm{b}>0$ can also be excluded.

Considering all of these observations the standard model of eq 2.2 remains a suitable format for characterizing the behavior of particle flux in experiments. By subtracting off the steady state solution from equation 2.5 we obtain the equation for the perturbed flux

$$
\tilde{\Gamma}_{r}(r)=\frac{1}{r} \int_{0}^{r}\left(\tilde{S}\left(r^{\prime}\right)-\frac{\partial \tilde{n}}{\partial t}\left(r^{\prime}\right)\right) r^{\prime} d r^{\prime} .
$$

In the core of a gas fuelled, high density Alcator $\mathrm{C}$ plasma the perturbed particle source term is negligible compared to the inertial term for any significant perturbation occurring on the equilibration time scale. By combining eqs. 2.8 and 2.2 and neglecting the perturbed particle source term we obtain an expression which can be used to determine the perturbed particle transport coefficients in the core of the Alcator $\mathrm{C}$ plasma from measurements of the density profile

$$
\mathbf{D}(r) \frac{\partial \tilde{n}(r, t)}{\partial r}+\mathbf{V}(r) \tilde{n}(r, t)=\frac{1}{r} \int_{0}^{r}\left(\frac{\partial \tilde{n}\left(r^{\prime}, t\right)}{\partial t}\right) r^{\prime} d r^{\prime} .
$$

Equation 2.9 is the basis for the analysis methods presented in chapter 3 .

\subsection{Consequences of Transport Coefficient De- pendences on Plasma Parameters}

The parameters which define a plasma must affect the transport processes and hence

the transport coefficients. The effects of such dependences on perturbed transport behavior will now be considered in detail. The convection and diffusion coefficients are assumed to be functions of $n, \nabla n, T$ and $\nabla T$. The perturbed transport equations for this case were published by Gentle [26] in cartesian coordinates. Other dependences can be included but the results given are quite instructive and include most of the important dependences. Gentle used cartesian coordinates to permit analytic evaluation of the effects. The effects will persist in cylindrical coordinates, with small 
modifications, but the increased complexity entailed in the numerical calculations would obscure their underlying causes [26].

The linearized expression for the perturbed particle flux is

$$
\begin{aligned}
-\tilde{\Gamma}= & D \frac{\partial \tilde{n}}{\partial x}+V \tilde{n}+\left(\tilde{n}_{x} \frac{\partial D}{\partial n_{x}}+\tilde{n} \frac{\partial D}{\partial n}+\tilde{T}_{x} \frac{\partial D}{\partial T_{x}}+\tilde{T} \frac{\partial D}{\partial T}\right) \frac{\partial n_{o}}{\partial x} \\
& +\left(\tilde{n}_{x} \frac{\partial V}{\partial n_{x}}+\tilde{n} \frac{\partial V}{\partial n}+\tilde{T}_{x} \frac{\partial V}{\partial T_{x}}+\tilde{T} \frac{\partial V}{\partial T}\right) n_{o}
\end{aligned}
$$

with

$$
A_{x} \equiv \frac{\partial A}{\partial x} .
$$

When there is no particle source the continuity equation is expanded into the following expression

$$
\begin{aligned}
\frac{\partial \tilde{n}}{\partial t}= & \left(D+\frac{\partial D}{\partial n_{x}} \frac{\partial n_{o}}{\partial x}+\frac{\partial V}{\partial n_{x}} n_{o}\right) \frac{\partial^{2} \tilde{n}}{\partial x^{2}}+\left(\frac{\partial D}{\partial T_{x}} \frac{\partial n_{o}}{\partial x}+\frac{\partial V}{\partial T_{x}} n_{o}\right) \frac{\partial^{2} \tilde{T}}{\partial x^{2}} \\
& +\left[V+\frac{\partial V}{\partial n} n_{o}+\frac{\partial D}{\partial n} \frac{\partial n_{o}}{\partial x}+\frac{d}{d x}\left(\frac{\partial V}{\partial n_{x}} n_{o}\right)+\frac{d D}{d x}+\frac{d}{d x}\left(\frac{\partial D}{\partial n_{x}} \frac{\partial n_{o}}{\partial x}\right)\right] \frac{\partial \tilde{n}}{\partial x} \\
& +\left[\frac{d V}{d x}+\frac{d}{d x}\left(\frac{\partial D}{\partial n} \frac{\partial n_{o}}{\partial x}\right)+\frac{d}{d x}\left(\frac{\partial V}{\partial n_{o}}\right)\right] \tilde{n} \\
& +\left[\frac{d}{d x}\left(\frac{\partial D}{\partial T_{x}} \frac{\partial n_{o}}{\partial x}\right)+\frac{\partial D}{\partial T} \frac{\partial n_{o}}{\partial x}+\frac{d}{d x}\left(\frac{\partial V}{\partial T_{x}} n_{o}\right)+\frac{\partial V}{\partial T} n_{o}\right] \frac{\partial \tilde{T}}{\partial x} \\
& +\left[\frac{d}{d x}\left(\frac{\partial D}{\partial T} \frac{\partial n_{o}}{\partial x}\right)+\frac{d}{d x}\left(\frac{\partial V}{\partial T} n_{o}\right)\right] \tilde{T}
\end{aligned}
$$

The spatial derivatives of the transport coefficients are evaluated through their implicit dependences upon plasma parameters. Note that the effective particle diffusion coefficient (term multiplying $\partial^{2} \tilde{n} / \partial x^{2}$ ) is modified from the equilibrium value $\mathrm{D}$, if the transport coefficients depend upon density gradients. The first three terms in brackets multiplying $\partial \tilde{n} / \partial x$ contribute to the perturbed convection velocity. The last three terms result from the spatial variation of the diffusion coefficient and do not contribute to the perturbed convection coefficient, contrary to the published claim [26]. This equation could be rewritten in the form

$$
\begin{aligned}
\frac{\partial \tilde{n}}{\partial t}= & \frac{\partial}{\partial x}\left(\left(D+\frac{\partial D}{\partial n_{x}} \frac{\partial n_{o}}{\partial x}+\frac{\partial V}{\partial n_{x}} n_{o}\right) \frac{\partial \tilde{n}}{\partial x}\right) \\
& +\frac{\partial}{\partial x}\left(\left(V+\frac{\partial D}{\partial n} \frac{\partial n_{o}}{\partial x}+\frac{\partial V}{\partial n} n_{o}\right) \tilde{n}\right)+\ldots
\end{aligned}
$$


The term in parenthesis multiplying $\partial \tilde{n} / \partial x$ is the effective diffusive coefficient. It has a spatial variation, but the overall behavior of the term is diffusive in nature. Empirically the volume integral of the left hand side of the above equation is correlated with the local values of perturbed variables at the flux surface to determine the transport coefficients in eq. 2.9. Fluxes driven by the perturbed local variables which correlate well in time with $\tilde{n}$ are effectively convective fluxes. The spatial variation of the perturbed diffusion coefficient, which multiplies $\nabla \tilde{n}$ in this equation, affects only the diffusive portion of the flux.

In theories of $\eta_{i}$ turbulence, resistivity-gradient-driven turbulence and perhaps drift wave turbulence, transport coefficients depend upon $\nabla T$. In order to examine the effect of this we consider the case where transport coefficients depend only on $\nabla T$. The effective diffusivity remains $\mathrm{D}$ in equation $2.11[26]$. The $\nabla T$ dependence can cause the perturbed temperature and density equations to become strongly coupled. Gentle [26] gives a highly instructive illustration of these effects by considering fast perturbations, where only the highest spatial derivatives need be considered, but retaining the full range of dependences of the transport coefficients. The perturbed equations for density and temperature are

$$
\begin{aligned}
\frac{\partial \tilde{n}}{\partial t}= & \left(D+\frac{\partial D}{\partial n_{x}} \frac{\partial n_{o}}{\partial x}+\frac{\partial V}{\partial n_{x}} n_{o}\right) \frac{\partial^{2} \tilde{n}}{\partial x^{2}}+\left(\frac{\partial D}{\partial T_{x}} \frac{\partial n_{o}}{\partial x}+\frac{\partial V}{\partial T_{x}} n_{o}\right) \frac{\partial^{2} \tilde{T}}{\partial x^{2}} \\
\frac{3}{2} \frac{\partial \tilde{T}}{\partial t}= & \left(\frac{T_{o}}{n_{o}}\right) \frac{\partial \tilde{n}}{\partial t}+\left(\chi+\frac{\partial \chi}{\partial T_{x}} \frac{\partial T_{o}}{\partial x}+1.5 T_{o} \frac{\partial U}{\partial T_{x}}\right) \frac{\partial^{2} \tilde{T}}{\partial x^{2}} \\
& +\left(\frac{\partial \chi}{\partial n_{x}} \frac{\partial T_{o}}{\partial x}+1.5 T_{o} \frac{\partial U}{\partial n_{x}}\right) \frac{\partial^{2} \tilde{n}}{\partial x^{2}}
\end{aligned}
$$

where $\chi$ is the thermal diffusivity and $U$ is the "heat pinch" velocity which is independent of the thermal transport from particle convection. The equations are cast into the form of the coupled oscillator problem. Two solutions, corresponding to a fast wave and slow wave are obtained. Numerical results were generated for the case when $\mathrm{D}$ and $\chi$ depend only on $\nabla T$. The effect of this coupling on the fast, primarily thermal root is that the wave diffuses more rapidly than the equilibrium diffusion rate. For realistic values of $\chi / D$ and $\eta$, relevant to experiment, the ratio of $\chi_{\text {eff }} / \chi$ is $\geq 2$. Thermal diffusivities calculated from sawtooth heat pulse propagation have 
been reported to be higher than equilibrium values by a similar ratio on a number of experiments. This could be considered as evidence that the transport is driven by temperature gradients [26]. The slow root always diffuses more slowly than $\mathrm{D}$, but for realistic values of $\chi / D$ and $\eta$ the modification to the apparent diffusivity is rather modest. A temperature perturbation accompanies the slow wave density perturbation, but it is always of opposite sign. When the coefficients depend upon $\mathrm{T}$ the effective particle convection velocity is modified because of the coupling between $\tilde{n}$ and $\tilde{T}$ in the slow root. The perturbed diffusion and convection coefficients are thus related to the functional forms $\mathbf{D}$ and $\mathbf{V}$ as

$$
\begin{aligned}
& D=D_{e q}+\frac{\partial \mathbf{D}}{\partial \frac{\partial n}{\partial r}} \frac{\partial n_{e q}}{\partial r} \\
& V=V_{e q}+\frac{\partial \mathbf{D}}{\partial n} \frac{\partial n_{e q}}{\partial r}+\frac{\partial \mathbf{V}}{\partial n} n_{e q}+\frac{\partial \mathbf{V}}{\partial T} \frac{\tilde{T}}{\tilde{n}} n_{e q}+\frac{\partial \mathbf{D}}{\partial T} \frac{\tilde{T}}{\tilde{n}} \frac{\partial n_{e q}}{\partial r}+\ldots
\end{aligned}
$$

The term from $\partial \mathbf{V} / \partial\left(\frac{\partial n}{\partial r}\right)$ is absent from the expression for $\mathrm{D}$ because any significant dependence of $\Gamma$ on $\partial n / \partial r$ is usually associated with $\mathbf{D}$ by definition. It is apparent that the relation between the perturbed and equilibrium convection velocities can be quite complex. Virtually any dependence of the transport coefficients on plasma parameters will result in a perturbed convection velocity different from the equilibrium value [26]. Theoretical expressions for the flux can be compared with values derived from gas modulation experiments through the expansions of equations 2.14 and 2.15 . The perturbed transport coefficients are themselves of fundamental significance: they describe how the system responds to a small perturbation.

\subsection{Experimental Considerations}

The gas modulation method for extracting perturbed particle transport coefficients will now be described. Detailed line-integrated measurements of the density profile evolution, necessary for this approach, are obtained on MTX with a 15-chord farinfrared interferometer (FIR) described in appendix D. It is desired that quasi-steadystate plasma conditions be established prior to the start of the perturbation. The gas valve flow is then modulated, creating density waves which propagate into the 
plasma. It is essential that the changes in the local density and density gradient be fractionally small in order for linearized analysis to be valid. Changes in $\mathrm{n}$ and $\nabla \mathrm{n}$ must not be correlated so that the convective and diffusive portions of the flux can be differentiated. Two general types of modulation can be employed, periodic modulation or a single gas puff. The first method offers the advantage that a variety of approaches can be used to analyze the data.

When periodic density modulation is employed the modulation frequency may not be chosen arbitrarily. If the modulation frequency is too slow, the density modulation will be quasi-static in nature and it will be impossible to separate the convective and diffusive components. If the modulation frequency is too high the diffusive term will cause the density wave to damp rapidly near the edge of the plasma, making it rather difficult to determine the central density variation accurately from a line-integrated measurement. This effect was examined by calculating the amplitudes and phases of the line integrals of the fundamental frequency density modulation component versus radius for various normalized frequencies and transport coefficient profiles. The spectral form of the particle transport equation

$$
i \omega \tilde{n}=\frac{1}{r} \frac{\partial}{\partial r}\left(r D \frac{\partial \tilde{n}}{\partial r}\right)+\frac{1}{r} \frac{\partial}{\partial r}\left(r^{2} \tilde{n} V / a\right)+\tilde{S}(r)
$$

was solved with the source modelled as

$$
\tilde{S}(r)=S_{o} \exp ^{i \omega t+(r-a) / \lambda_{s}} .
$$

For the case when $D$ is taken as constant and $V(r)=V_{o} \times r / a$ this can be expressed in the following non-dimensional form

$$
i \tilde{n}(\rho) \beta=\frac{1}{\rho} \frac{\partial}{\partial \rho}\left(\rho \alpha^{-1} \frac{\partial \tilde{n}}{\partial \rho}\right)+\frac{1}{\rho} \frac{\partial}{\partial \rho}\left(\rho^{2} \tilde{n}\right)+\underline{\tilde{S}}(\rho)
$$

with $\alpha=\mathrm{aV} / \mathrm{D}, \beta=\omega \mathrm{a} / \mathrm{V}, \underline{\tilde{S}}=\tilde{S} \mathrm{a} / \mathrm{V}$ and $\rho=\mathrm{r} / \mathrm{a}$. The amplitudes and phases of the line integrated perturbed density components are shown in fig. 2.1 for a variety of values of $\beta$, and $\alpha=1$. The optimum normalized frequency for modulation falls in the range $2 \leq \beta \leq 10$. For moderate density MTX plasmas $\left(\bar{n}_{e} \sim 1.4 \times 10^{20}\right)$ the optimum modulation frequency range was found to be $5-15 \mathrm{~Hz}$. 

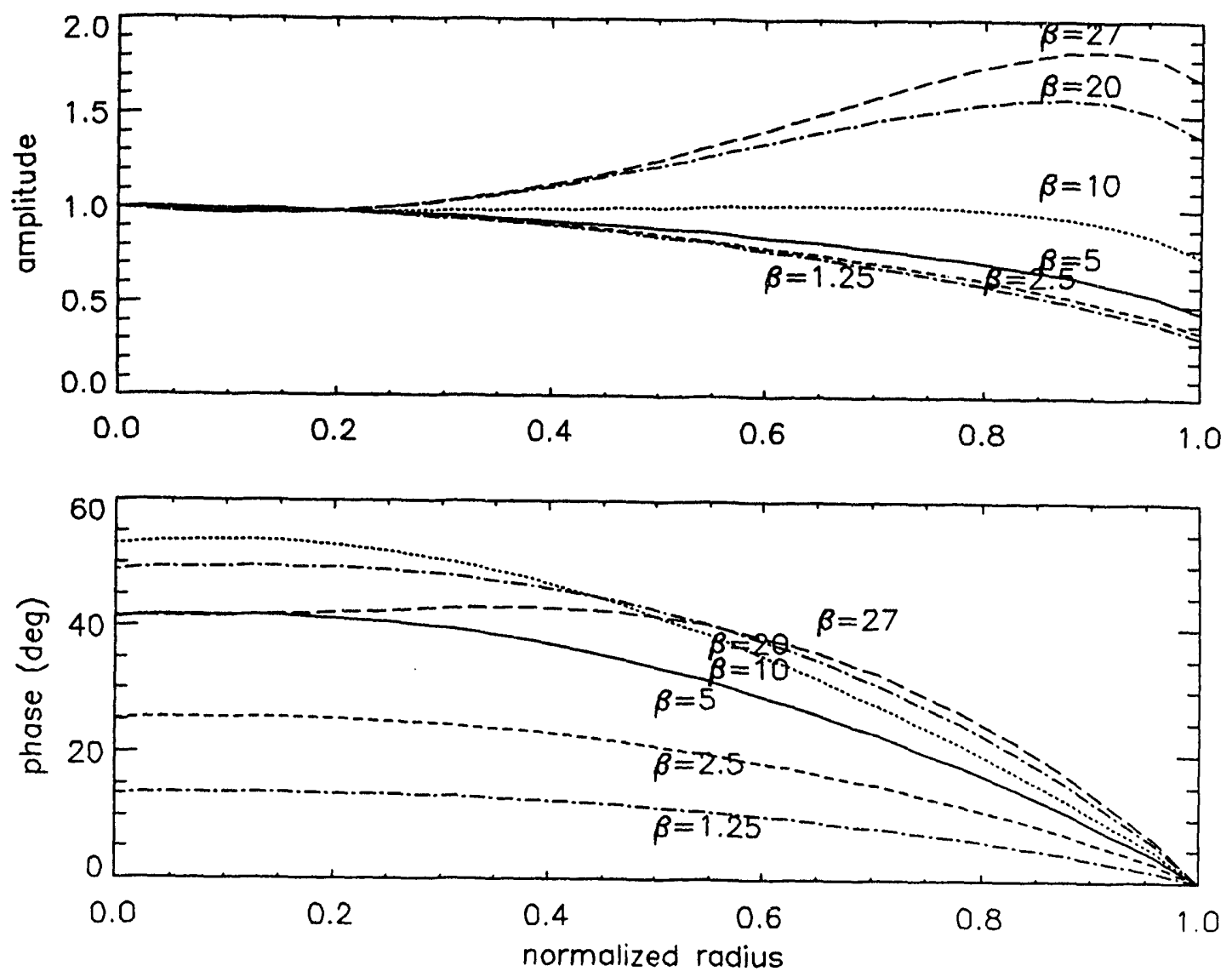

Figure 2.1: Amplitude and phases of chord measurements vs. radius with $\alpha=1$. 


\subsubsection{Stability of Plasma Position}

From the outset the need to maintain steady plasma position throughout the modulation period was recognized. The vertical field required for a stable tokamak plasma is given by [47]

$$
B_{v}=\frac{\mu_{o} I_{p}}{4 \pi R}\left(\ln \frac{8 R}{a}+\beta_{p o l}+\frac{\ell_{i}}{2}-1.5\right)
$$

and thus depends most strongly upon the plasma current. During early gas modulation shots, when the plasma current changed substantially during the modulation period, the plasma position could undergo excursions larger than $8 \mathrm{~mm}$. This amount was unacceptably large for the purpose of this experiment. This amount of plasma motion induces changes in the chord signals which are not small compared to those associated with subtle changes occurring in the profile due to the gas puff. In addition there are substantial changes in the nature of the limiter interaction with the edge plasma. The occurrence of all of these changes at once makes it difficult if not impossible to separate out accurately the changes in the core density profile. Since the OH power supplies on MTX lack current feedback capability the supply programmers were adjusted until, eventually, the current was held nearly steady through the plateau. Variations in plasma current as small as $1 \%$ during the plateau were obtained, as shown in fig. 2.2. Once the plasma current was held steady the plasma position remained quite stable during the modulation. Fig 2.3 shows the plasma position for shot 14034 moves out just $2 \mathrm{~mm}$ following a gas puff before being stabilized by the feedback circuit. This was the maximum displacement observed during modulation when the plasma current was held nearly constant. On most shots no detectable change in plasma position occurred during modulation. Changes in the interferometer signals due to this small amount of motion were sufficiently small that they could be ignored during the data analysis.

The flux surface geometry itself can change during gas modulation. The magnitude of the Shafronov shift, estimated from theory and derived from self-consistent equilibrium calculation using plasma data, as described in sec. 3.2 , is quite small on Alcator C. For typical plasmas the value at the center relative to the last closed flux 


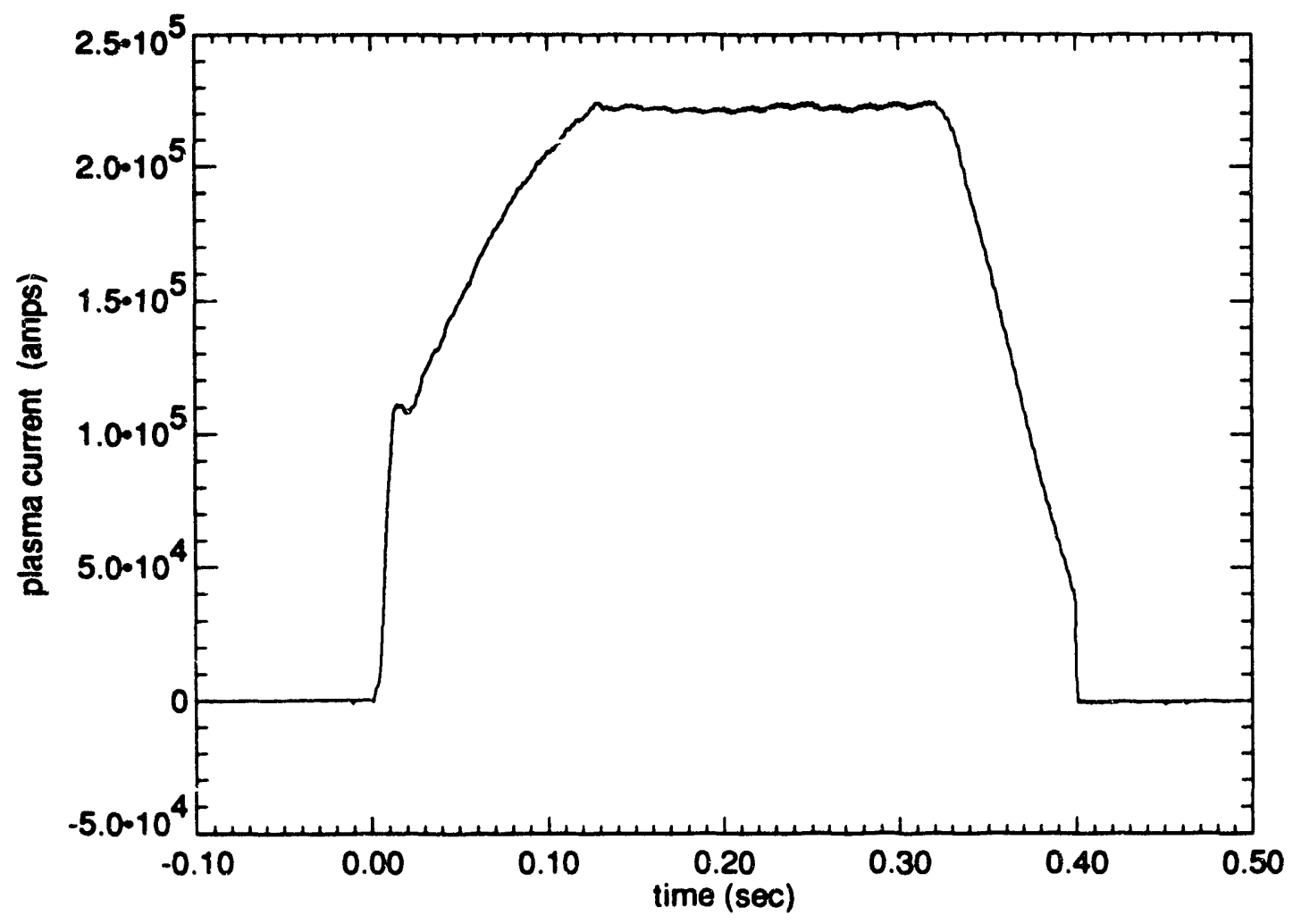

Figure 2.2: Nearly constant plasma current during the plateau of shot 13552

SHOT 14034
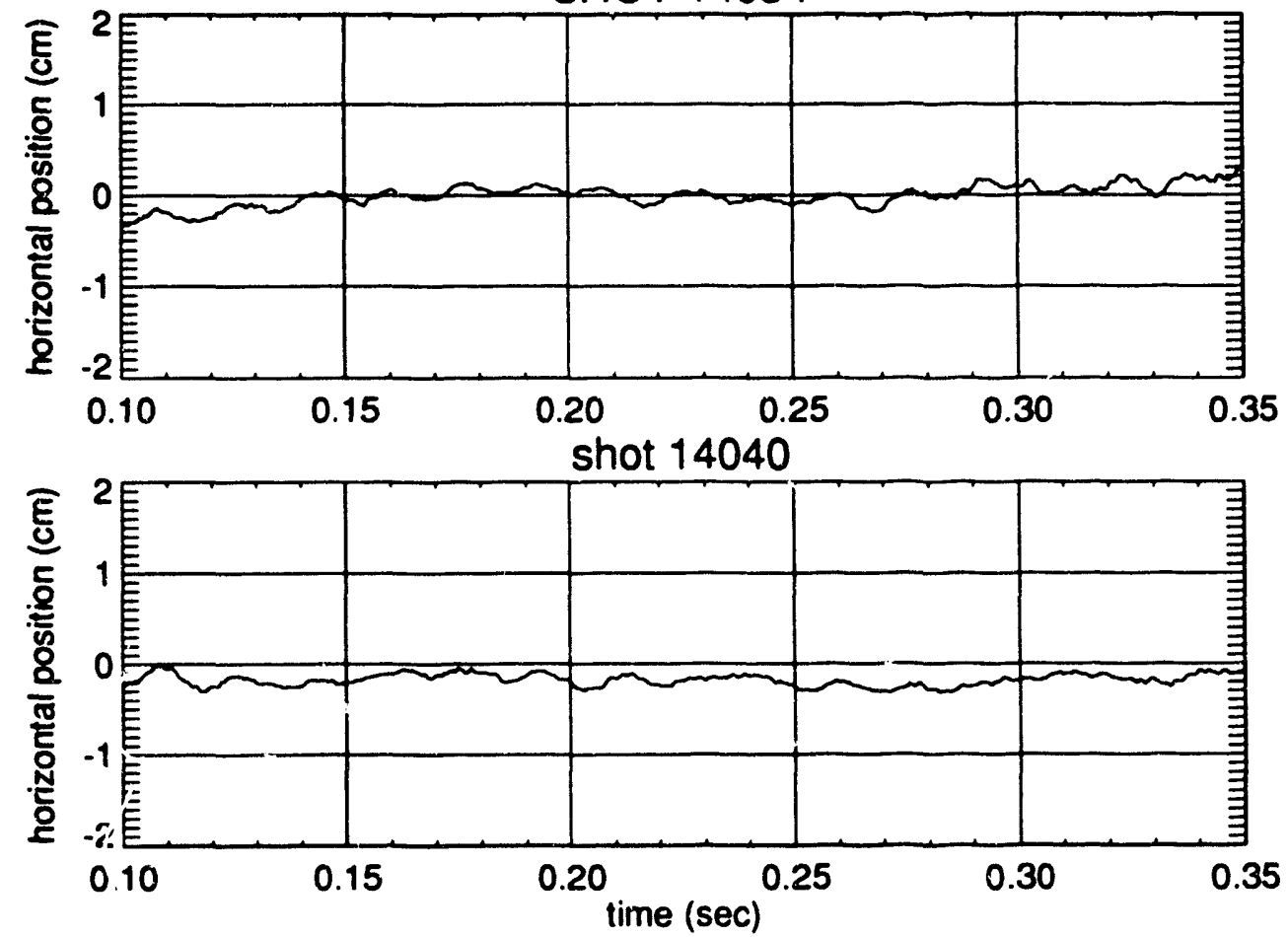

Figure 2.3: Plasina pusition during shots 14034, 14040. A gas puff occurs at 260 msec. 


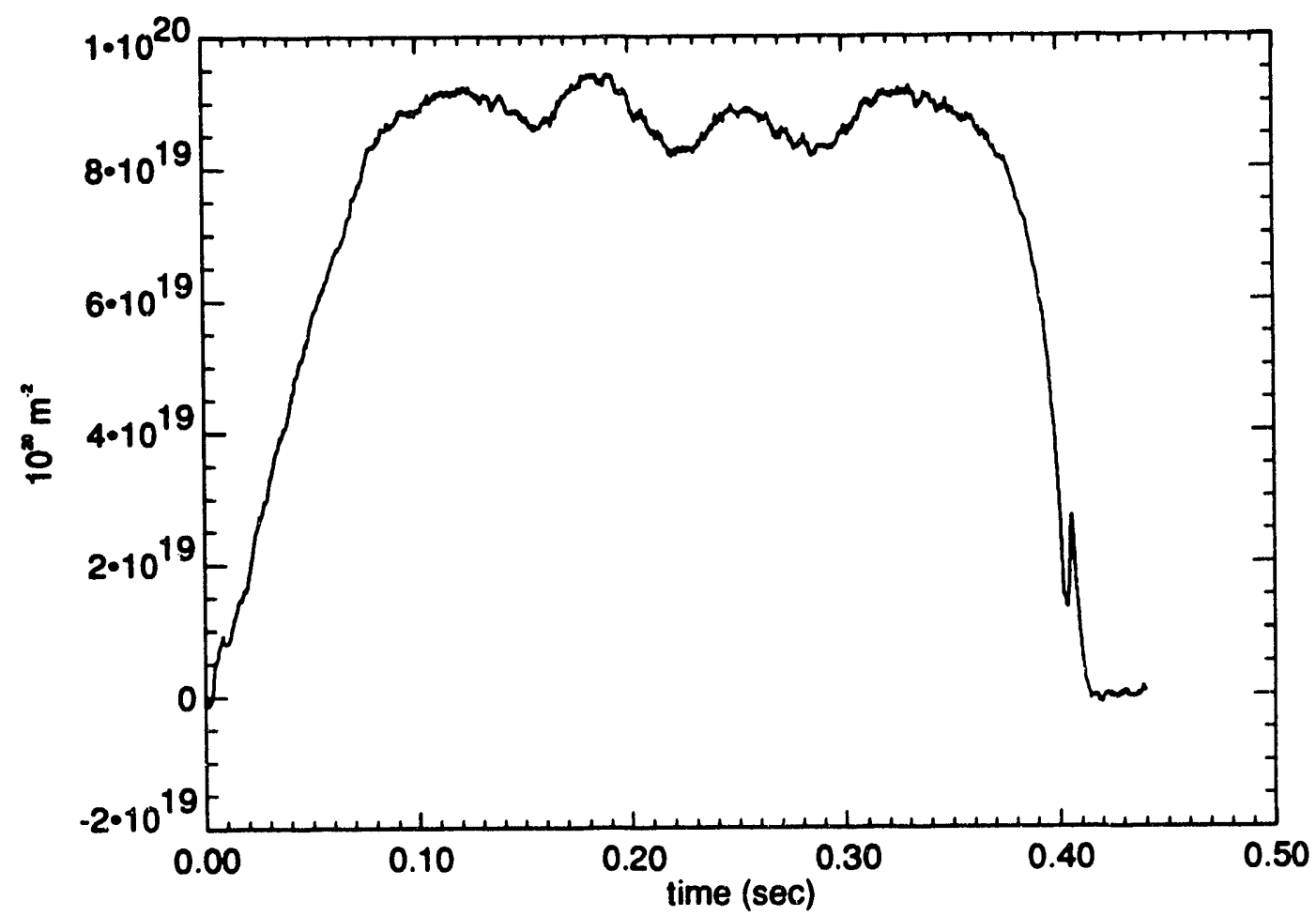

Figure 2.4: Line-averaged density during periodic gas modulation on shot 13524 .

surface ranges from $1.2-1.8 \mathrm{~cm}$. In these experiments the values of $\beta_{p}$ were modest and the changes in $\beta_{p}$ associated with gas puffing were quite small. Thus the overall changes in flux surface geometry associated with gas puffing should be negligibly small in these experiments.

The shot length on MTX was limited nominally to a total length of some $400 \mathrm{msec}$. As shown in fig. 2.2 the plasma current ramp up, following breakdown, lasts about 130 msec. After allowing $\sim 2$ energy confinement times for profiles to reach a quasi-steady state, periodic modulation at $15 \mathrm{~Hz}$ commences, as shown in fig. 2.4. Therefore the maximum length of time over which gas modulation about a quasi-steady state can be conducted is limited to $\sim 200 \mathrm{msec}$ in MTX.

\subsubsection{Plasma Response to Gas Modulation}

Because of the subtle nature of the modulations desired, the limited amount of time available during a shot and the difficulties associated with operating near the density limit, precise control of the gas fuelling valve was imperative. In order to facilitate 


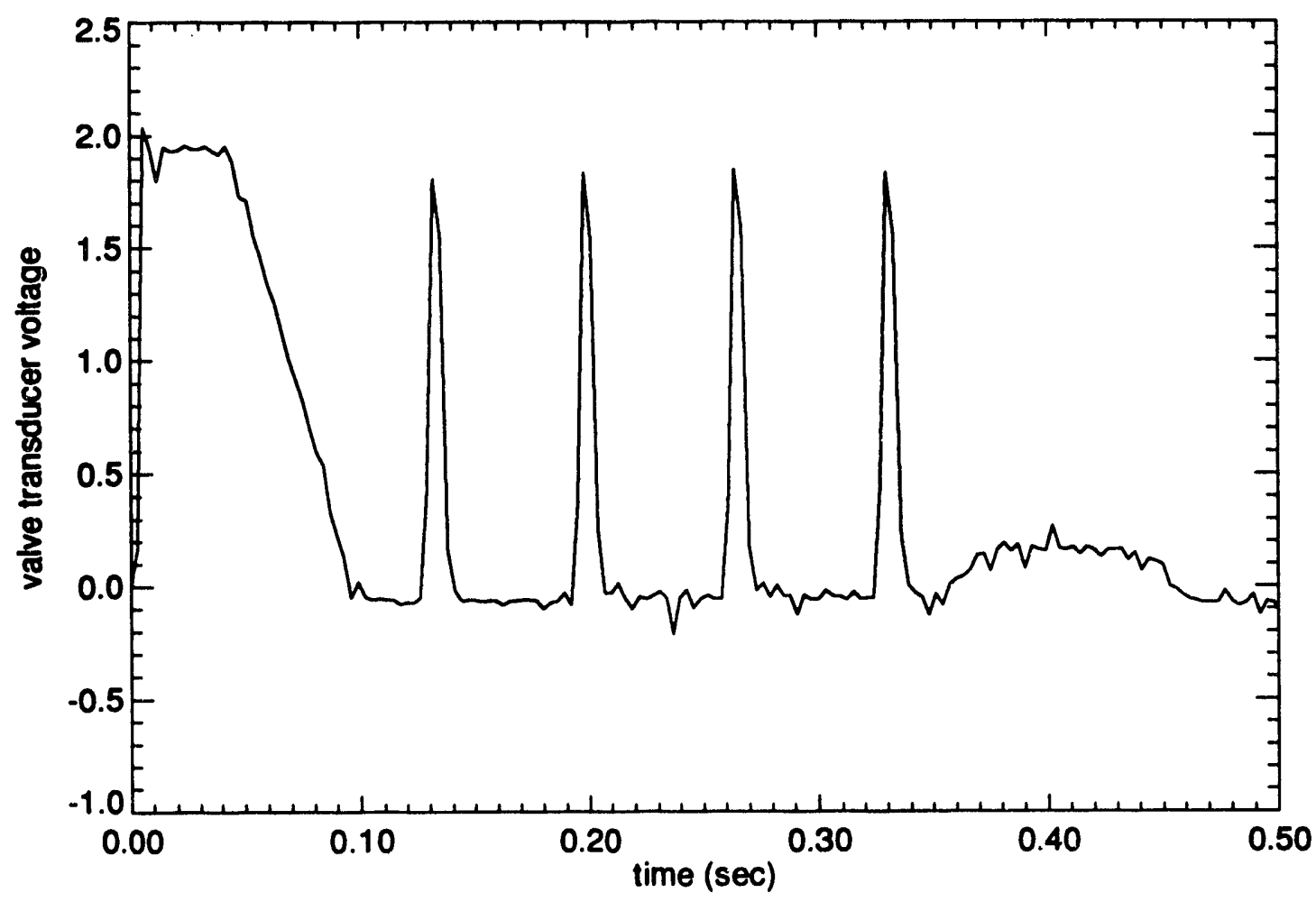

Figure 2.5: Gas valve flow with large amplitude, low duty cycle modulation.

operations for gas modulation experiments, a computer-controlled gas fuelling system, described in Appendix D, was installed on MTX.

The flexibility offered by this system proved valuable in these experiments. During operations at high densities the gas fuelling system was adjus'ed to bring the plasma density up quickly during the initial phases of the shot in order to maximize the limited time available for gas modulation. Periodic modulation with relatively little variation in average density could only be achieved for densities above $1 \times 10^{20} \mathrm{~m}^{-3}$ when the gas valve waveform had large amplitude puffs with a rather low duty cycle, as shown in fig. 2.5. At higher dersities the periodic modulation technique could not be used on Alcator C. The plasma responded more slowly to gas puffs as the density was raised into the saturated ohmic confinement regime, lowering the optimum frequency range for modulation. With the slower plasma response characteristic of the high densities a longer time was required to bring the density profile to a quasisteady state. Recyciing increased at higher density, evidenced by an increase in the $\mathrm{D}_{\alpha}$ signal. The characteristic time for the density to return to its prior level, once 


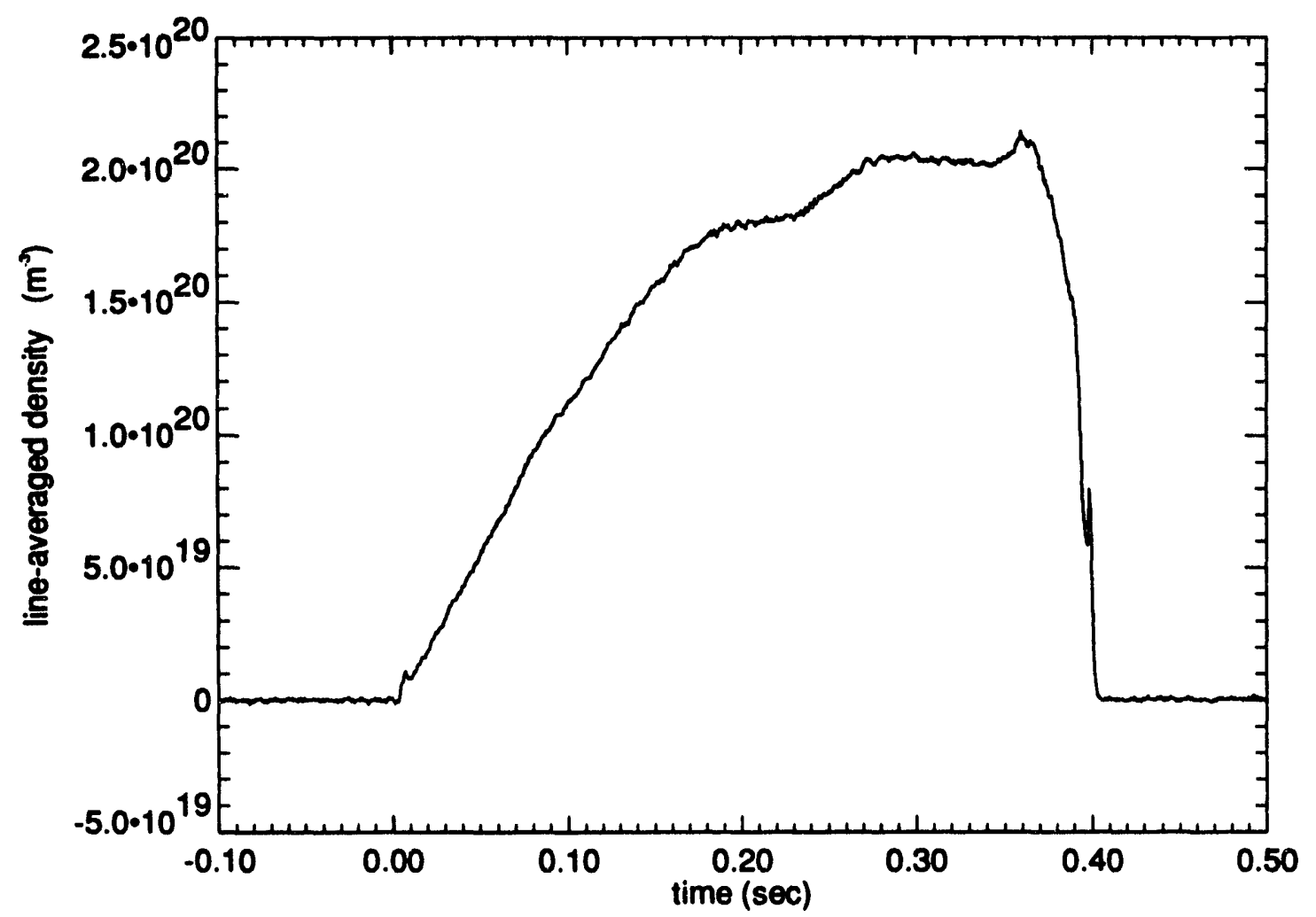

Figure 2.6: Line density during shot 13546 with a single gas puff modulation at 230 msec.

the gas valve closed following a gas puff, lengthened considerably with increasing density. The overall effect was that the period required between gas puffs exceeded the length of time during the shot effectively available for modulation. Only one option remained for high density shots: a single gas puff late in the steady state portion of the discharge, as shown in fig. 2.6

Single puff modulation shots were repeated a total of 8-10 times in order to achieve measurements with a good signal to noise ratio. With periodic modulation a few periods were completed on each shot, so that the total number of shot repetitions required was reduced to 4-6. The gas modulation shots were highly reproducible so that these requirements did not pose a problem. Density limit studies were pursued at low values of plasma current, with values of $q_{a}=(4.8,5.7)$, so that stable plasmas were produced even near the density limit, and refraction of the FIR beam was diminished.

Other difficulties became manifest as the density limit was approached. Marfes are characterized by large amplitude density fluctuations with rather small density 
gradient scale lengths. Whenever Marfe regions intersect an interferometer chord on MTX, they cause rapid, large amplitude changes in the beam refraction. The interferometer phase lock is lost, rendering the channel useless it if persists. At sufficiently high density the Marfe would encircle the plasma, as detached plasma conditions were approached. This would result in the breakup of all interferometer chords, instead of just the innermost ones, as normally occurs during Marfe activity. The plasma background density and puff amplitude were adjusted precisely on shots near the density limit to avoid these effects and to obtain good data as close to the density limit as possible.

Some degree of $60 \mathrm{~Hz}$ component is always visible on interferometer and visible bremsstrahlung array chords which are tangent to the outer portions of the plasma. This is due to a slight wobble in the horizontal position of the plasma caused by 60 $\mathrm{Hz}$ ripple in the vertical field coil current. This periodic component has a tendency to become more pronounced at densities approaching the limit density. This effect was inferred to be caused by the change in position of the edge flux surfaces relative to the limiter caused by the $60 \mathrm{~Hz}$ wobble. The resulting periodic change in the edge plasma-limiter interaction can result in periodic modulation of the local values of the poloidally asymmetric density at the limiter. It is significant because the periodic component will correlate between FIR chords in the regression analysis creating an unphysical contribution to the perturbed flux and variation of local variables. If this contribution is sufficiently large (i. e. comparable in magnitude to the variation from the true flux) it can adversely affect the results of the analysis. Because this component of the signal always retained its well defined $60 \mathrm{~Hz}$ frequency it could be effectively removed from most of the data through the application of a digital nonrecursive $60 \mathrm{~Hz}$ bandstop filter. Only shot data in which the $60 \mathrm{~Hz}$ component could be highly suppressed through filtering were used for the determination of transport coefficients. 


\section{Chapter 3}

\section{Method of Data Analysis}

Unlike the electron temperature profile, which can be measured locally from electron cyclotron emission, the density profile is obtained from the inteferometer on MTX in the form of a line-integrated measurement, complicating the data analysis. A procedure for obtaining a density profile from the chord data which incorporates a self-consistent refraction correction is derived in this chapter. Analysis routines based upon linear equations are outlined which are appropriate for determining small changes in the profiles during modulation. Separate versions are introduced which use spectral and linear regression methods to determine particle transport coefficients. With use of simulated data, the influence of spline fitting performed by the routines as well as the particle source term and filtering of the signal are considered.

\subsection{Processing of FIR Signals}

Before the interferometer signals can be analyzed "fringe shifts" and periodic noise must be removed. At times the interferometer phase comparator loses its phase lock, predominantly due to the effect of Marfe-like activity. If the loss of phase lock is only instantaneous, as is often the case, then the resulting fringe shift can be removed using the continuous nature of the time variation of the line-integrated density signal. Segments of data from both sides of a fringe shift are fitted to a cubic polynomial with an arbitrary step change connecting them using a least squares fitting routine. 

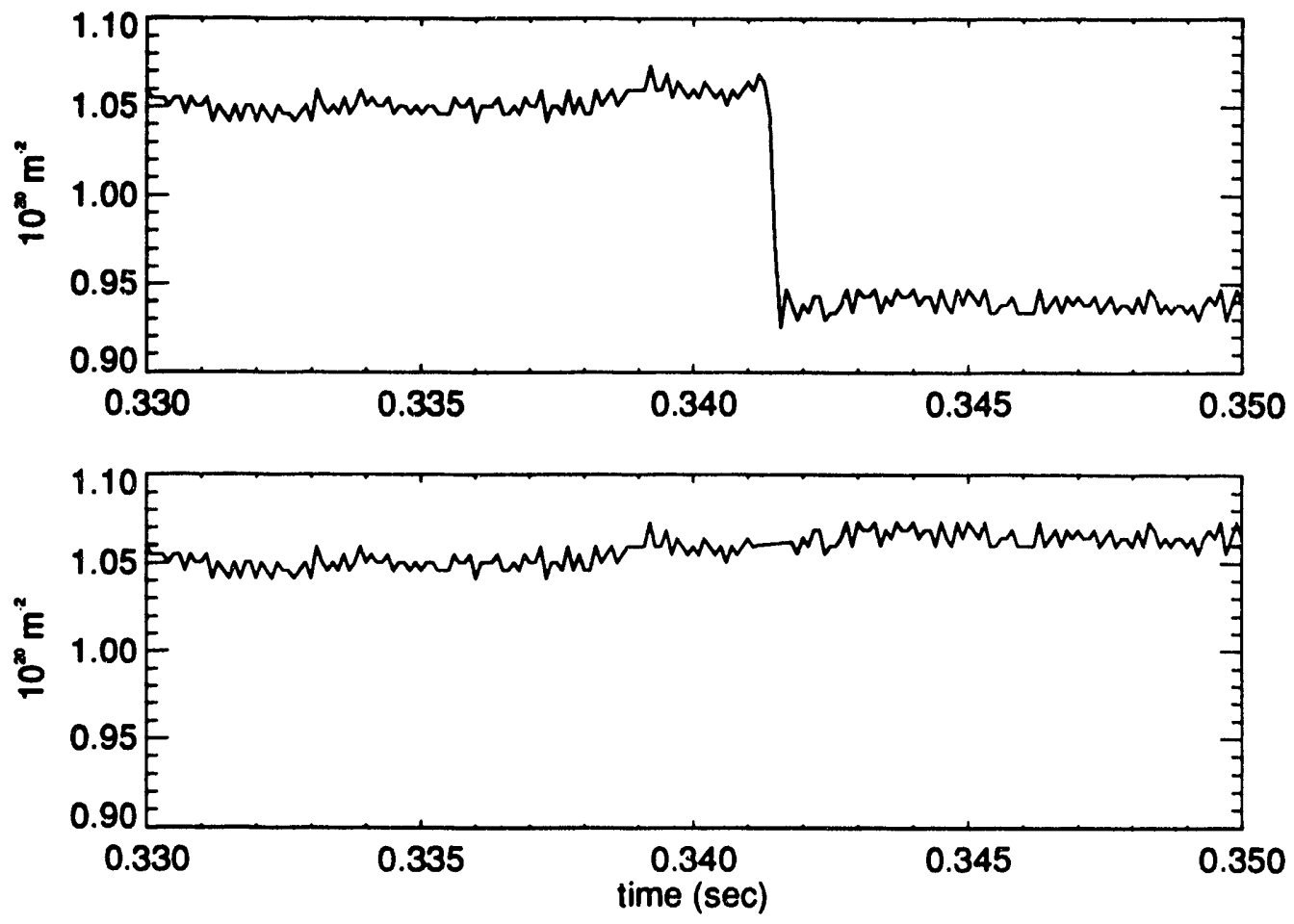

Figure 3.1: FIR signal before and after fringe removal

As shown in figure 3.1 the method is highly effective in removing individual fringe shifts of short duration $(<1 \mathrm{msec})$.

In addition to the $60 \mathrm{~Hz}$ component discussed in the last chapter harmonics of $120 \mathrm{~Hz}$, also related to the power supply, are present in the signals and must also be filtered out. Sometimes an $100 \mathrm{~Hz}$ component, caused by the operation of the $\mathrm{CO}_{2}$ laser power lock, is present. This $100 \mathrm{~Hz}$ component is very small and has a negligible effect upon these measurements. Because the information in the FIR signal associated with particle transport is predominantly in the low frequency range of the spectrum $(5-15 \mathrm{~Hz})$, the high frequency portion of the signal is dominated by noise. It is advantageous to maximize the signal to noise ratio with use of a low pass filter.

A causal composite filter with stop bands at 60 and $110 \mathrm{~Hz}$ and a low pass cutoff at $90 \mathrm{~Hz}$ was optimized for this application. It is described in Appendix E. The characteristic cutoff frequencies of this filter were sufficiently high that the low frequency particle transport information in the signal was not significantly distorted. An example of this is shown in 3.2 where unprocessed FlR data and the filtered output are 


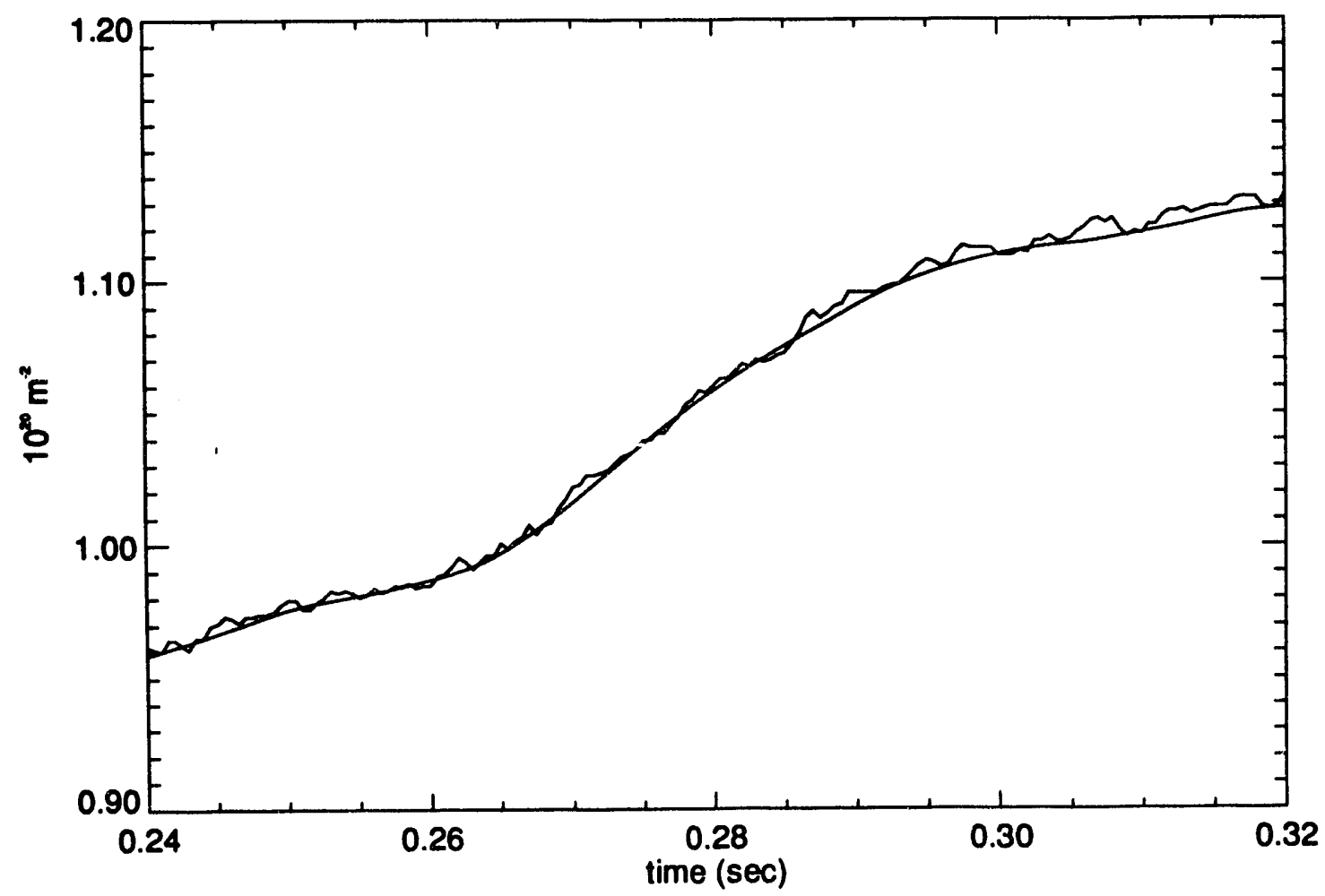

Figure 3.2: Superimposed FIR chord signals with and without filtering during gas puff

superimposed. The filtered signals are then used to obtain a "background" density profile at a reference time by fitting the profile to a set of basis functions with a self-consistent refraction correction as will be described in section 3.3 .

\subsection{Equilibrium Simulation}

The flux surface geometry for the MTX configuration has been analyzed with an equilibrium solution to the Grad-Shafronov equation calculated using the ACCOME code $[29,30]$. Although the code is capable of modelling a tokamak plasma equilibrium with inductive current drive as well as with non-inductive current drive sources such as neutral beams, lower-hybrid and bootstrap effects, non-inductive current drive mechanisms were absent in the simulation results given here.

The actual coil geometry was input so that an accurate equilibrium field could be calculated. The equilibrium field on MTX is produced by two sets of coils, EF1 and 


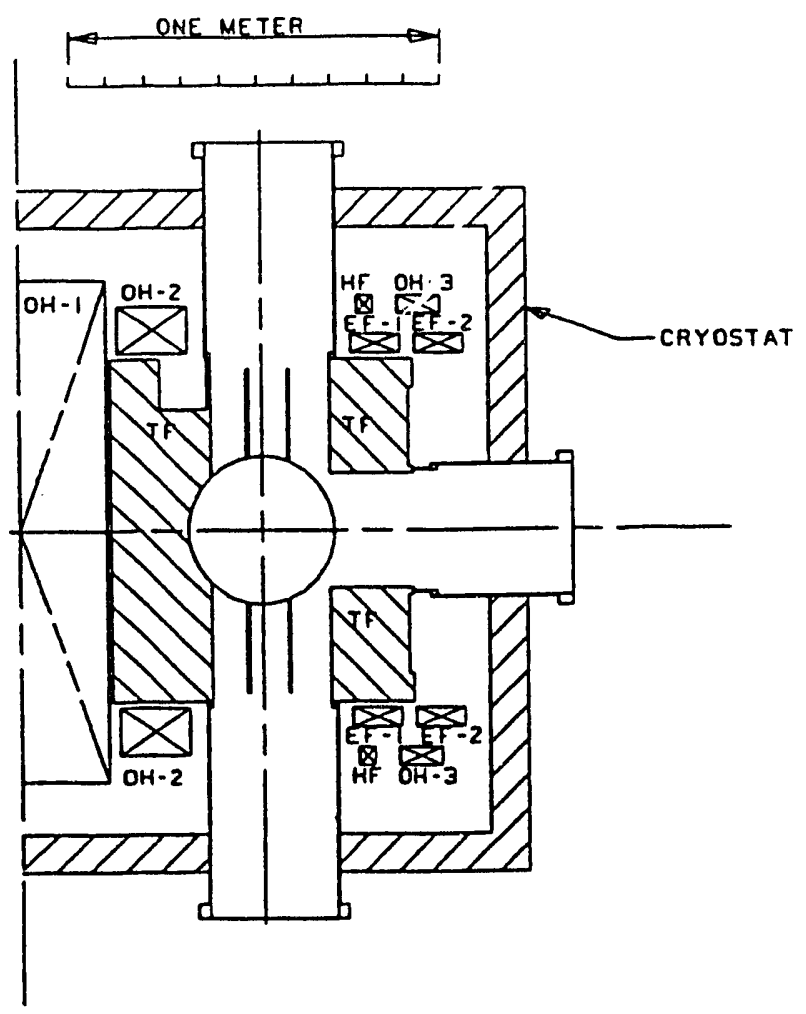

Figure 3.3: Cross section of the MTX Tokamak

EF2. These are illustrated in Fig. 3.3. EF1, which has a radius of $0.95 \mathrm{~m}$, is located at vertical positions of $\pm 0.5 \mathrm{~m}$ and has 61 turns. EF2 is located at the same height with a radius of $1.12 \mathrm{~m}$ and has 52 turns. The toroidal field strength is taken to be simply inversely proportional to major radius. The inductive electric field is taken to be uniform with a value consistent with the input plasma current. Spitzer conductivity is used to determine the current profile. Plasma density and temperature profiles are input using the following parametrizations:

$$
\begin{aligned}
& n(\psi)=n_{o}\left[1-(1-\psi)^{\alpha_{n}}\right]^{\beta_{n}} \\
& T_{e}(\psi)=T_{e o}\left[1-(1-\psi)^{\alpha_{t}}\right]^{\beta_{t}}
\end{aligned}
$$

The following typical MTX plasma parameters were used in the calculation: 

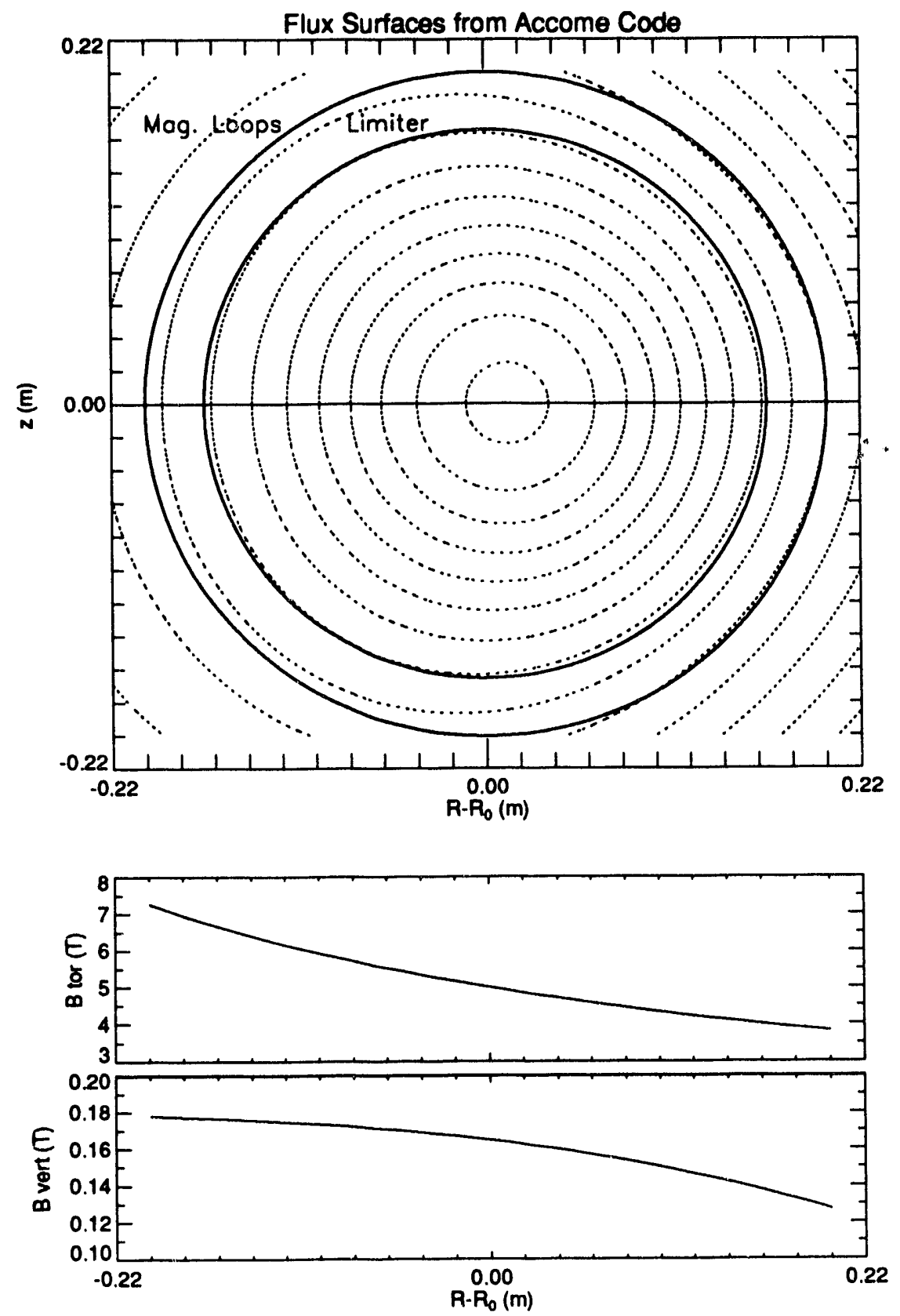

Figure 3.4: MTX surfaces of constant flux calculated by ACCOME. 


$$
\begin{aligned}
\text { EF1, EF2 current } & =1803 \mathrm{kA} \\
B_{t} & =5 \mathrm{~T} \\
I_{p} & =350 \mathrm{kA} \\
\alpha_{n} & =0.8, \quad \beta_{n}=1.0 \\
n_{o} & =3.0 \times 10^{20} \mathrm{~m}^{-3} \\
\alpha_{t} & =1.2, \quad \beta_{t}=1.0 \\
T_{e} & =1.2 \mathrm{keV}, \quad T_{i o}=1.0 \mathrm{keV} \\
Z_{\text {eff }} & =1.5 .
\end{aligned}
$$

Several significant output parameters are

$$
\begin{aligned}
\Delta(0) & =1.31 \mathrm{~cm} \\
q(0) & =0.85 \\
q(a) & =3.4 \\
\beta_{p} & =0.35 \\
\ell_{i} & =1.24 \\
\beta_{t} & =0.0025
\end{aligned}
$$

Profiles of $\mathrm{n}(\mathrm{R}), \mathrm{T}_{e}(\mathrm{R}), \mathrm{B}_{z}(\mathrm{R})$ and $\psi(\mathrm{R})$ for this equilibrium are shown in Fig. 3.5 plotted vs major radius at the midplane. A contour plot of the calculated constant flux surfaces is shown in Fig. 3.4 along with the radial variation of the toroidal and vertical field strengths at the tokamak midplane. If the vertical field was constant, solutions to the Grad-Shafronov equation would be concentric offset circular flux surfaces. In Alcator $\mathrm{C}$, as in most tokamaks, the vertical fie'd decreases with increasing major radius for improved vertical stability. The effect of this is to introduce a slight triangularity into the flux surface geometry. The offsets of the flux surfaces are important for this analysis. The slight deviation of flux surface geometry from circularity is too small to be significant. 

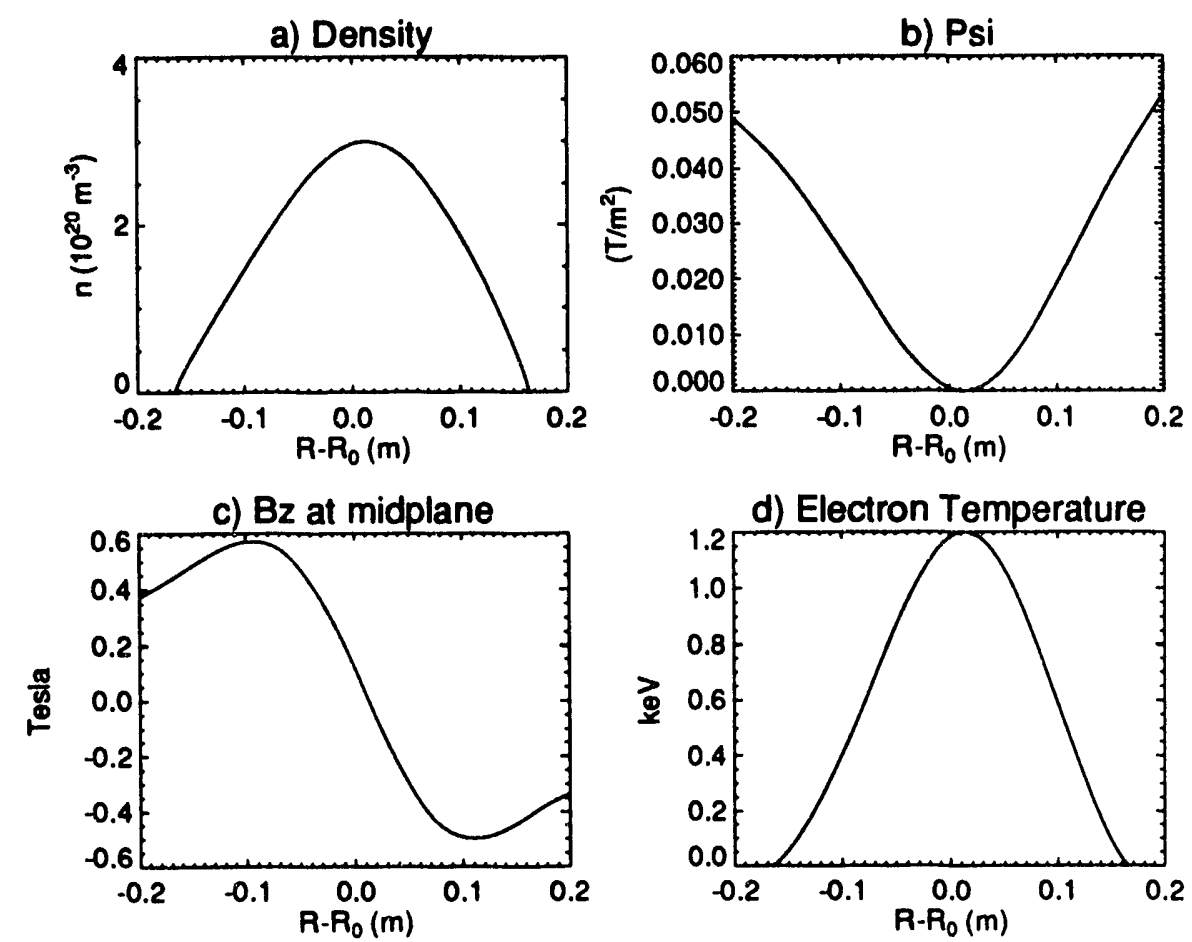

Figure 3.5: Plasma profiles used in the ACCOME simulation.

\subsection{Profile Determination}

Since the plasma density measured by the interferometer is a line-averaged measurement, some method must be employed to determine the plasma density profile from the data. The Abel [31] inversion is one well known method of accomplishing this. The chord integral, expressed in cylindrical radial coordinates is

$$
N_{\ell}(x)=2 \int_{r_{o}}^{a} n(r) \frac{r d r}{\sqrt{r^{2}-x^{2}}} .
$$

The Abel inversion is determined by

$$
n_{(r)}=-\frac{1}{\pi} \int_{r}^{a} \frac{\partial N_{\ell}(x)}{\partial x} \frac{d x}{\sqrt{x^{2}-r^{2}}} .
$$

The integral here is sensitive to the details of $\frac{\partial N}{\partial x}$, particularly close to the singularity at $r=x$. This poses certain difficulties, particularly because $N_{\ell}(x)$ is only known at a fixed number of chord locations. This method has been extended to include the effects of density asymmetries [32] as well as Shafronov shifts [49, 50, 51]. 
For the present analysis the method of profile fitting adopted is inspired by the methods used for tomographic reconstruction of mode structures using soft $\mathrm{x}$-ray data. Fourier-Bessel basis functions have been shown to be well suited for use with this method $[52,53]$.

In the interior of the plasma, where classical transport rates parallel to the magnetic field are very high, we would not expect to see any substantial density asymmetry on a given flux surface. Near the edge, low plasma temperature and interaction with the limiter can lead to substantial poloidal asymmetries. Measurements with DENSEPACK [54], a poloidal array of Langmuir probes, in Alcator $\mathrm{C}$ show strong poloidal asymmetries in the plasma density at minor radii greater than or equal to the limiter radii which persist for all values of $\frac{\bar{n}_{\ell}}{l_{p}}$.

The ratio of the maximum to poloidal average of density was examined at three radii $(\mathrm{r}=16.5,16.8,17.2 \mathrm{~cm})$ for a wide range of values of $\frac{\bar{n}_{l}}{I_{p}}$. This ratio was nearly insensitive to $\frac{\bar{n}_{\ell}}{I_{p}}$ with only the $\mathrm{r}=16.8 \mathrm{~cm}$ data showing a slight increase with $\frac{\bar{n}_{\ell}}{I_{p}}$. The values for the ratio fell in the range $n_{e}^{\max } /\left\langle n_{e}\right\rangle_{\text {ave }} \approx 1.5-2.5$ for all three radii. The ratio of the minimum to average density shows more of a dependence with $\frac{\bar{n}_{l}}{l_{p}}$, generally lowering at high $\frac{\bar{n}_{t}}{l_{p}}$, except for the $\mathrm{r}=17.2 \mathrm{~cm}$ data which remains fixed to a very low value $\sim .05-.15$. Overall there is only a slight increase in the normalized density asymmetry at high values of $\frac{\bar{n}_{p}}{l_{p}}$.

Studies with DENSEPACK also showed that the Marfe is the result rather than the cause of asymmetries in the edge plasma [54]. The hypothesis of a preferentially cooler, Marfe-prone region existing at the upper-inside poloidal location was verified by experiment. The density at the limiter radius was found to be strongly poloidally asymmetric, independent of a Marfe. In fact, a Marfe was detected as a relatively weak poloidal density perturbation [54].

A threshold criterion for Marfe occurrence on Alcator C was found to be $\frac{\bar{n}_{f}}{I_{p}}$ $\left[10^{12} \frac{\mathrm{cm}^{-3}}{k A}\right] \geq 0.6$. A scatter plot of data from discharges in Marfe and non-Marfe regimes show no obvious difference in the magnitude of normalized density asymmetry [54]. In addition the angular positions of the density extrema remain the same for any value of $\frac{\bar{n}_{l}}{l_{p}}$. Also, data from shots in the Marfe versus non-Marfe regimes do 
not indicate any shift in the overall density asymmetry.

Only three of the fifteen interferometer chords are far enough inside of the magnetic axis to be useful sources of information on in-out poloidal asymmetries. These chords are lost when Marfes are present, such as in high-density discharges. Good fits to the interferometer profiles can be obtained without introducing any poloidal asymmetry into the form of the resulting density profile. For these reasons only poloidally symmetric terms are included in the analysis.

As we saw from the ACCOME simulation, the flux surfaces of Alcator $\mathrm{C}$ are well represented as circular with a Shafronov shift. In this analysis this geometry is represented by the following equation

$$
\left(x+\delta_{o}+\epsilon_{o}\left(1-\rho^{\alpha}\right)\right)^{2}+y^{2}=\rho^{2}
$$

with $\mathrm{x}$ the horizontal coordinate, $\mathrm{y}$ the vertical coordinate, $\rho$ the flux surface radius, $\epsilon_{o}$ the Shafronov shift and $\delta_{0}$ the plasma offset. All of these coordinates are normalized to the radius of the last closed flux surface with the origin at the magnetic axis of the machine. The Shafronov shift is determined by an equilibrium fit to polarimeter, interferometer and other data [55]. The normalized profile of the Shafronov shift (1$\left.\rho^{\alpha}\right)$ compares well with the equilibrium profiles from a wide variety of gas modulation shots considered for $\alpha=2.75$. The magnitude of the Shafronov shift of the center relative to the last closed flux surface is typically $\sim 1.4 \mathrm{~cm}$ and varies over a fairly narrow parameter range for the gas modulation shots considered. The plasma is usually offset slightly to the inside of the torus on MTX as the operators believe this results in the most stable plasma operation.

The density profile is represented by the following set of basis functions

$$
\begin{array}{ll}
\rho \leq 1 & n(\rho)=\left.n\right|_{\rho=1}+\sum_{i=1}^{4} n_{i} J_{o}\left(\lambda_{i} \rho\right) \\
\rho>1 & n(\rho)=\left.n\right|_{\rho=1} e^{-\left(\frac{(\rho-1)}{\xi_{n}}\right)}
\end{array}
$$

with $\lambda_{i}$ is the ith zero of the zerc order Bessel function $\mathrm{J}_{o}$ and $\xi_{n}$ is the density decay length in the scrape-off layer. The choice of 4 Bessel functions was motivated by the observation that this was the optimum number to yield a sufficiently general profile 
fit without substantial spurious details. The exponential density decay behind the limiter was measured in Alcator $\mathrm{C}$ with the DENSEPACK array [54].

\subsubsection{Refraction Corrections}

Refraction corrections are often neglected on most devices because they are typically of order $1 \%$. On MTX they are significant for two basic reasons: 1) MTX can operate at rather high densities which, along with the small plasma minor radius, results in large aensity gradients; 2 ) the detectors are located sufficiently far from the plasma such that substantial chord offsets result from small refraction angles. The refraction correction is implemented here be ause high-density plasmas are to be studied and it is desired that the density profile be obtained with the greatest accuracy.

Refraction error analysis has been derived for an interferometer which utilizes a collimated beam [56]. The situation on the MTX interferometer is different because the probe beam is expanded into a continuous sheet, interrupted only by the blockage of the port ribs. The effect of plasma refraction is to shift the effective chord radius viewed by the detector. The geometry is illustrated in Fig. 3.6. A ray in the laser beam directly incident upon the detector in the absence of plasma is denoted by segment AB. Since the phase velocity of the wave exceeds $c$ in the plasma the refracted beam, represented by segment $A C$, is bent outward. Because the refraction angle is small, the path is approximately straight in the plasma. Using this approximation the refraction angle for a chord located a distance $\mathrm{x}$ from the midplane is derived in Appendix $B$, with the result given by

$$
\Theta=-\frac{x}{n_{c}} \int_{x}^{a} \frac{\partial n}{\partial r} \frac{d r}{\sqrt{r^{2}-x^{2}}}
$$

where $\mathrm{n}_{c}=c^{2} \epsilon_{o} m 4 \pi^{2} /\left(\lambda^{2} e^{2}\right)$ is the cutofi density. For $\lambda=184 \mu \mathrm{m} \mathrm{n}_{c}$ equals $326 \times 10^{20} \mathrm{~m}^{-3}$.

Plasma refraction causes the chord positions viewed by the detector to be displaced inward by the distance

$$
\Delta x=H \sin \Theta \approx H \Theta
$$




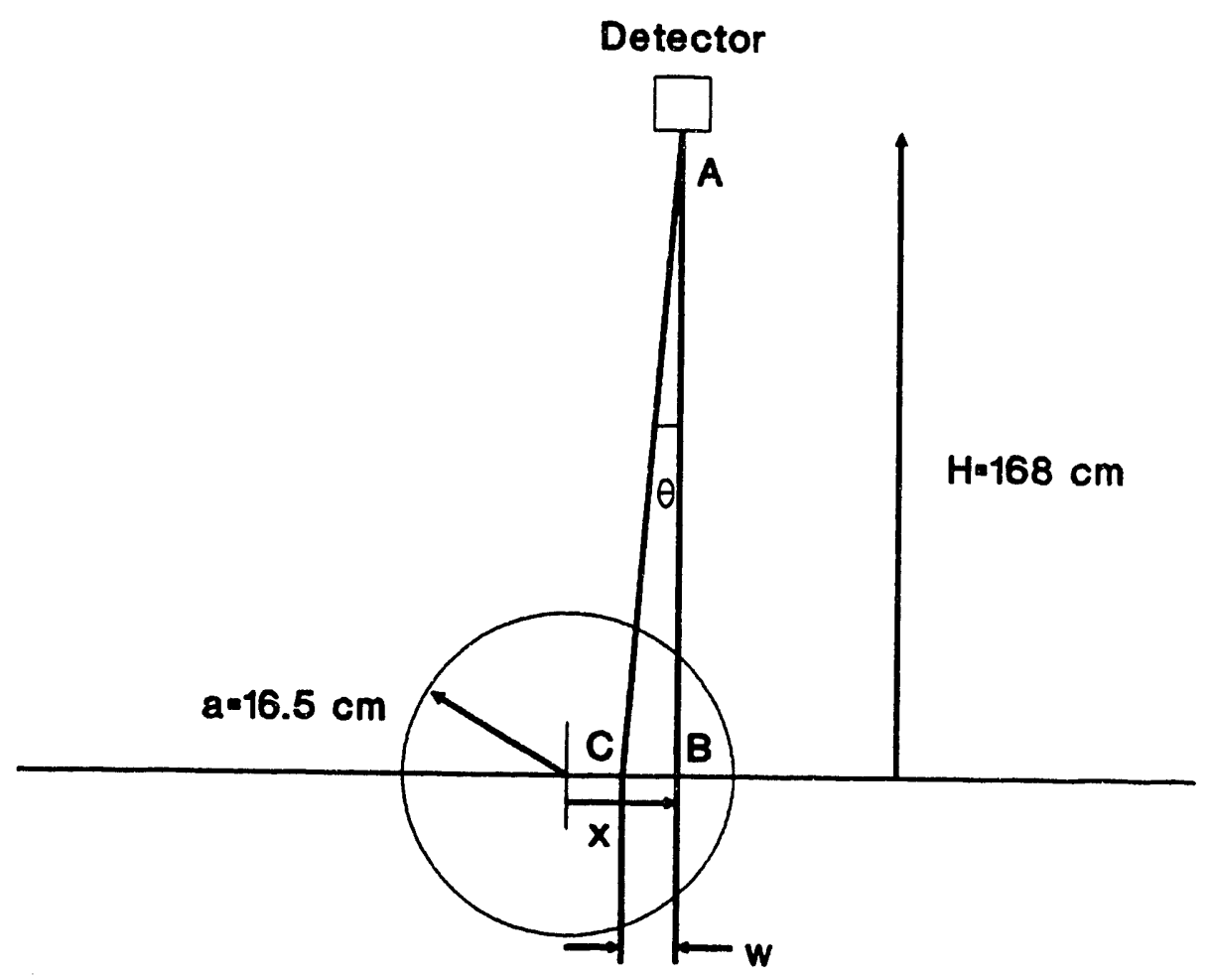

Figure 3.6: Effect of refraction on FIR ray geometry

As the refraction angle increases during the shot from 0 to $\Theta$, the path length of the chord is lengthened by the amount

$$
\Delta \ell=H \sec \Theta-H \approx \frac{H \Theta^{2}}{2} .
$$

Note that the increase in path length tends to offset somewhat the change due to the shift of the chord position. The maximum refraction angles are usually less than 0.01 $\operatorname{rad}\left(0.6^{\circ}\right)$ for the range of densities in the discharges studied here.

It must be mentioned that the blockage of portions of the beam by the port ribs adds to the diffraction caused by the finite beam size. The first detectors on the inside edges of the outer slots are particularly affected by this since in the absence of diffraction, refraction typically moves their viewing position behind the port ribs. This is a possible source of inaccuracy in the measurement. Profiles of chord measurements at fixed points in time do not show any noticeably different behavior in these channels than the others; spline fits easily pass through these points without any inflection. 


\subsubsection{Calculation of Chord Integrals}

The region of the cross section with $\rho<1$ is divided into 50 discrete flux surfaces, separated by equal spacing of $\Delta \rho$. Along a chord the quantity $\Delta y$, a discrete path length element, is most conveniently evaluated in matrix form given by

$$
\begin{aligned}
& \Delta y_{i j}=2\left(\left[\left(x_{i}+\delta_{o}+\epsilon_{o}\left(1-\rho_{j}^{\alpha}\right)\right)^{2}-\rho_{j}^{2}\right]^{\frac{1}{2}}-\left[\left(x_{i}+\delta_{o}+\epsilon_{o}\left(1-\rho_{j-1}^{\alpha}\right)\right)^{2}-\rho_{j-1}^{2}\right]^{\frac{1}{2}}\right) \\
& \Delta y_{i j}=2\left[\left(x_{i}+\delta_{o}+\epsilon_{o}\left(1-\rho_{j}^{\alpha}\right)\right)^{2}-\rho_{j}^{2}\right]^{\frac{1}{2}}, \quad \text { at the equator } \\
& \Delta y_{i j}=0, \quad \Delta y_{i j}^{2}<0
\end{aligned}
$$

The contributions to the line integrals are then evaluated by multiplying the vector of $\mathrm{n}(\rho)$, the density at the center of each ring, by the matrix of $\Delta y$ 's. In a similar manner the region of $\rho>1$ is divided into 30 radial zones, extending many times the scrape-off layer thickness outside of the last closed flux surface. The $\Delta y$ matrix, generated from the same equation for the flux surface geometry, multiplies the vector of the density basis function values generated from equation 3.5 for $\rho>1$. The equation for the total contribution to a chord from the profile of basis functions is

$$
\begin{aligned}
N_{L, i}= & a\left(1-\delta_{o}\right) \sum_{j}\left(n_{(\rho=1)}+\sum_{k} n_{k} J_{o}\left(\lambda_{k} \rho_{j}\right)\right) \Delta y_{i j}^{\text {interior }} \\
& +a\left(1-\delta_{o}\right) \sum_{j} n_{(\rho=1)} e^{-\left(\frac{\left(\rho_{j}-1\right)}{\xi_{n}}\right)} . \Delta y_{i j}^{\text {exterior }}
\end{aligned}
$$

The refraction angle (eq. 3.6) for each chord is also expanded in terms of the contributions of the basis functions yielding the following expression:

$$
\Theta_{i}=\frac{x_{1}}{n_{c}} \sum_{j=1}^{50} \sum_{m=1}^{4} \lambda_{m} n_{m} J_{1}\left(\lambda_{m} \rho_{j}\right) \frac{\Delta y_{i j}^{\text {interior }}}{\rho_{j}}+\frac{x_{1}}{n_{c}} \sum_{j=1}^{50} n_{(\rho=1)} e^{-\left(\frac{\left(\rho_{j}-1\right)}{\xi_{n}}\right)} \frac{\Delta y_{i j}^{\text {exterior }}}{\xi_{n} \rho_{j}} \text {. }
$$

The refraction corrections are calculated for the given parameters at the initial chord locations. The chord positions and path lengths are then adjusted and the refraction correction re-calculated at the new positions. A self-consistent correction is obtained by iterating the procedure just, a few times. Fit parameters for the chord data are obtained using a non-linear least squares fitting routine based on the conjugate gradient 
method [57]. The routine minimizes the quantity

$$
\chi^{2}=\sum_{i}\left[\frac{1}{\sigma_{i}^{2}}\left(N_{i}-N_{f}\left(x_{i}\right)\right)^{2}\right]
$$

where $\mathrm{N}_{i}$ is the ith measured line integral, $\mathrm{N}_{f}\left(\mathrm{x}_{i}\right)$ is the calculated fit at the position of the ith chord and $\sigma_{i}$ is the standard deviation of measurement uncertainty for the ith chord. The routine converges relatively quickly, but is too slow for the purpose of fitting data from a large number of time steps. For this reason the geometry and refraction corrections determined from the background fit are used with a separate linear routine which analyzes the time variation of the profiles.

For a small perturbation of the density profile about this background profile the changes in the refraction correction are second order effects and can be neglected. This is illustrated with the following argument. Consider a small perturbation $\epsilon \zeta(\mathrm{r})$ to the background density $n_{o}(r)$ which results in the profile

$$
n(r)=n_{o}(r)+\epsilon \zeta(r)
$$

where $\zeta(\mathrm{r})$ is a continuous function and $\epsilon$ is taken to be small. The phase at the detector viewing along a ray with wavenumber $k_{o}$ introduced as the ray traverses the plasma at position $\mathrm{x}$ is given by

$$
\phi=\frac{k_{o}}{n_{c}} \int_{x}^{a} n(r) \frac{r d r}{\sqrt{r^{2}-x^{2}}} .
$$

We consider only the portion of the change in the phase of the detector signal due to the change in the refraction correction due to $\epsilon \zeta$. This is evaluated for small changes $\epsilon \rightarrow 0$ as

$$
\lim _{\epsilon \rightarrow 0}=\left.\frac{\partial \phi}{\partial \epsilon}\right|_{\epsilon=0}=\frac{\partial \phi}{\partial x} \frac{d x}{d \Theta} \frac{\partial \Theta}{\partial \epsilon}-k_{o} \frac{\partial \Delta \ell}{\partial \Theta} \frac{\partial \Theta}{\partial \epsilon} .
$$

The first term is the change in phase due to the change of chord position in the plasma, while the second is the change in signal phase due to the change of the path length. This is equal to

$$
\left.\frac{\partial \phi}{\partial \epsilon}\right|_{\epsilon=0}=-\frac{\partial}{\partial x}\left(\frac{k_{0}}{n_{c}} \int_{x}^{a} \frac{n(r) r d r}{\sqrt{r^{2}-x^{2}}}\right) H \frac{\partial \Theta}{\partial \epsilon}-k_{o} H \Theta \frac{\partial \Theta}{\partial \epsilon}
$$


By integrating the term in brackets by parts and using the fact that only regions with finite density contribute to the refraction integral one obtains

$$
\left.\frac{\partial \phi}{\partial \epsilon}\right|_{\epsilon=0}=H \frac{\partial \Theta}{\partial \epsilon}\left[\frac{\partial}{\partial x}\left(\frac{k_{0}}{n_{c}} \int_{x}^{a} \frac{\partial n(r)}{\partial r} \sqrt{r^{2}-x^{2}} d r\right)-k \Theta\right]
$$

which by Leibnitz rule reduces to

$$
\left.\frac{\partial \phi}{\partial \epsilon}\right|_{e=0}=H \frac{\partial \Theta}{\partial \epsilon} k_{0}\left[-\frac{x}{n_{c}} \int_{x}^{a} \frac{\partial n(r)}{\partial r} \frac{d r}{\sqrt{r^{2}-x^{2}}}-\Theta\right] .
$$

Inspection of equation 3.6 reveals that the first term in brackets is simply equal to $\Theta$, so that the expression above vanishes. Thus the contribution due to refraction is stationary for small, continuous variations in the density profile. Contributions from the change in the refraction correction can also be inferred to be second order in $\epsilon$ through Fermat's principle. Since the refraction corrections and plasma position can be treated as constants through the period of modulation, the time-dependent inversion problem for density perturbations reduces to a linear set of equations, greatly simplifying the analysis.

\subsection{Analysis in the Time Domain}

\subsubsection{Spline Fit}

With this approach to the time dependent problem chord data is fit at $1 \mathrm{msec}$ intervals to a spline under tension. The rontine utilized for the spline fit computes the fit function $f$ given abscissae $x_{i}$ and ordinates $y_{i}, i=1, \ldots, n$ by minimizing

$$
\sum_{i=1}^{n-1}\left[\left.\frac{d^{2} f}{d x^{2}}\right|_{x_{i}}+\sigma^{2}\left(\left.\frac{d f}{d x}\right|_{x_{i}}-\frac{\Delta y_{i}}{\Delta x_{i}}\right)\right]
$$

over all functions $f$ with two continuous derivatives such that the sum of the squares of $\left(f\left(x_{i}\right)-y_{i}\right) / d_{i}$ is less than a given constant $s$. The coefficient $d_{i}$ is the standard deviation error associated with the ith chord signal. It is taken here as the greater of either the rms noise level of the chord during a period prior to plasma initiation or the uncertainty in the chord calibration taken as $1 \%$ of the signal amplitude. When $\mathrm{s}$ is set equal to zero and $\sigma=0$ an ordinary cubic spline fit passing through the 
data points results. As $s$ is increased the spline fit is increasingly smootheu. A value of $s$ equal to the number of chords yields the smoothest resulting fit function which passes through the error bars of the data points. The parameter $\sigma$ is the tension factor which determines the degree to which the first derivative part of the smoothing is emphasized. Some degree of smoothing of the fit function can be desirable. A fit function constrained to pass through the data points can amplify errors in the chord signals, particularly those near the gaps created by the port ribs. Excessive smoothing will distort the information contained in the fit by moving the fit function further away from the data points.

We must consider how the edge points of the spline fit are handled. The interferometer chord with the greatest major radius is just outside the limiter, tangent to a minor radius of $16.75 \mathrm{~cm}$ from the machine axis. The effective position with plasma is shifted inward somewhat by refraction, and crosses a region of non-negligible density. Measurements with the DENSEPACK array [54] showed that the poloidally averaged density scrape-off length behind the limiter radius varies with density from $\sim 0.4$ to $0.6 \mathrm{~cm}$. The density scrape-off length is, however, highly asymmetric and has a maximum at a poloidal location just above the equator on the outside of the machine, where it is approximately $1 \mathrm{~cm}$. The density at the limiter is poloidally asymmetric as well, implying that significant asymmetry exists on closed flux surfaces just inside the limiter. For a line averaged density of $2 \times 10^{20} \mathrm{~m}^{-3}$ the density measured at the limiter varies between $2 \times 10^{19} \mathrm{~m}^{-3}$ at the outside of the torus to $1.1 \times 10^{20} \mathrm{~m}^{-3}$ just inside either the bottom or top of the vessel [54]. Therefore it would be incorrect to set $n_{e}(a)=0$ as a boundary condition. The outer edge boundary condition for the spline fit of chord data is obtained by adding an extra chord with a constant amplitude equal to $5 \%$ that of the chord at $+16.75 \mathrm{~cm}$ at a reference time and at 1.12 times its normalized radius. This point is given a rather small weighting by making its $d_{i}$ several times larger than that for neighboring data points. The factor 1.12 was chosen to allow the flux surface grid to extend sufficiently beyond the outermost chord radius so that the profile could fall off smoothly. For comparison the poloidal average of measured density at $\mathrm{r}=17.6 \mathrm{~cm}[54]$ is typically a few percent of the central value. 
When all of the inside interferometer chords are usable, the chord of minimum major radius corresponds to a position of $-11.75 \mathrm{~cm}$ inside from the machine axis. There is a large gap between this chord and the inner plasma boundary, increased by the effect of refraction which is pronounced in this region. No extrapolation across this large region is attempted. Instead the chord data at minimum minor radius is taken as the inner boundary condition. For higher density shots typical of this study, the innermost useful chord is at $-2.75 \mathrm{~cm}$ because the three innermost chords are lost due to the effects of Marfes.

\subsubsection{Matrix Inversion}

Using equation 3.3 for the flux surface geometry along with the plasma position determined from the background profile fit, a grid of 50 flux surfaces is produced with equal spacings in $\rho$ so that the normalized radius of the largest flux surface is tangent with the outer boundary location at the plasma equator. Equations for the line-integrated density are generated at two positions between each location where each adjacent pair of flux surfaces intersect the equator. This is done for the whole region spanning the width of the spline fit to form a path length matrix DY. The corresponding positions of the detectors are calculated using the refraction corrections determined from the density profile obtained from the background fit. The resulting set of matrix equations

$$
\mathbf{N}(\mathbf{r})=\mathbf{D Y} \mathbf{n}(\rho)
$$

is over determined as the total number of chord positions where the spline fit is evaluated is greater than 100 compared to 50 flux surface grid points. This system is solved using singular value decomposition which, when applied to an over determined system is equivalent to determining the least-squares solution to the equations [59]. Once the system is solved using singular value decomposition the vectors $\mathrm{n}(\rho)$ are quickly computed for each time step using a back substitution routine. 


\subsubsection{Multidimensional Linear Regression Analysis}

The density and density gradient are calculated by averaging groups of four adjacent values from the inverted profiles. This averaging process tends to smooth out errors introduced through the spline fitting process. It results in twelve radial positions, which is considered an optimum value and is similar to the number of chords available for measurement. The coefficients $V(r), D(r)$ are determined by applying multidimensional linear regression analysis at each radial position for many time slices during the density perturbation according to

$$
\mathbf{D}(r) \frac{\partial \tilde{n}(r, t)}{\partial r}+\mathbf{V}(r) \tilde{n}(r, t)=\frac{1}{r} \int_{0}^{r}\left(\frac{\partial \tilde{n}\left(r^{\prime}, t\right)}{\partial t}\right) r^{\prime} d r^{\prime}
$$

taking $\tilde{n}$ and $\partial \tilde{n} / \partial r$ as independent variables. Details of the mathematics are given in Appendix C.

\subsection{Impact of Particle Source Term}

The significance of the particle source term neglected in eq. 3.20 is now analyzed quantitatively with calculations performed with the ONETWO transport code, used to analyze transport in tokamak plasmas. In the analysis mode used here, profiles of electron density, electron temperature and $\mathrm{Z}_{\text {eff }}$ are input along with other plasma parameters. Among the large number of parameters output by the code are profiles of neutral particle density, temperature and the particle source term. The code models a wide range of atomic physics phenomena including charge exchange, radiative recombination and impact ionization mechanisms using measured cross sections in the calculation of the neutral density profile. The overall magnitude of the particle source profile is scaled so as to match the input global particle confinement time. An estimate of this parameter is obtained from DENSEPACK data [54]. Using poloidally averaged electron temperature and density profiles in the scrape-off layer and using the assumption that the scrape-off layer plasma flows at the speed of sound to the limiter, the total particle source rate for the steady state plasma can be estimated as

$$
\int \Gamma \cdot d S \approx 4 \pi c_{s} n_{a} \lambda_{n} a .
$$




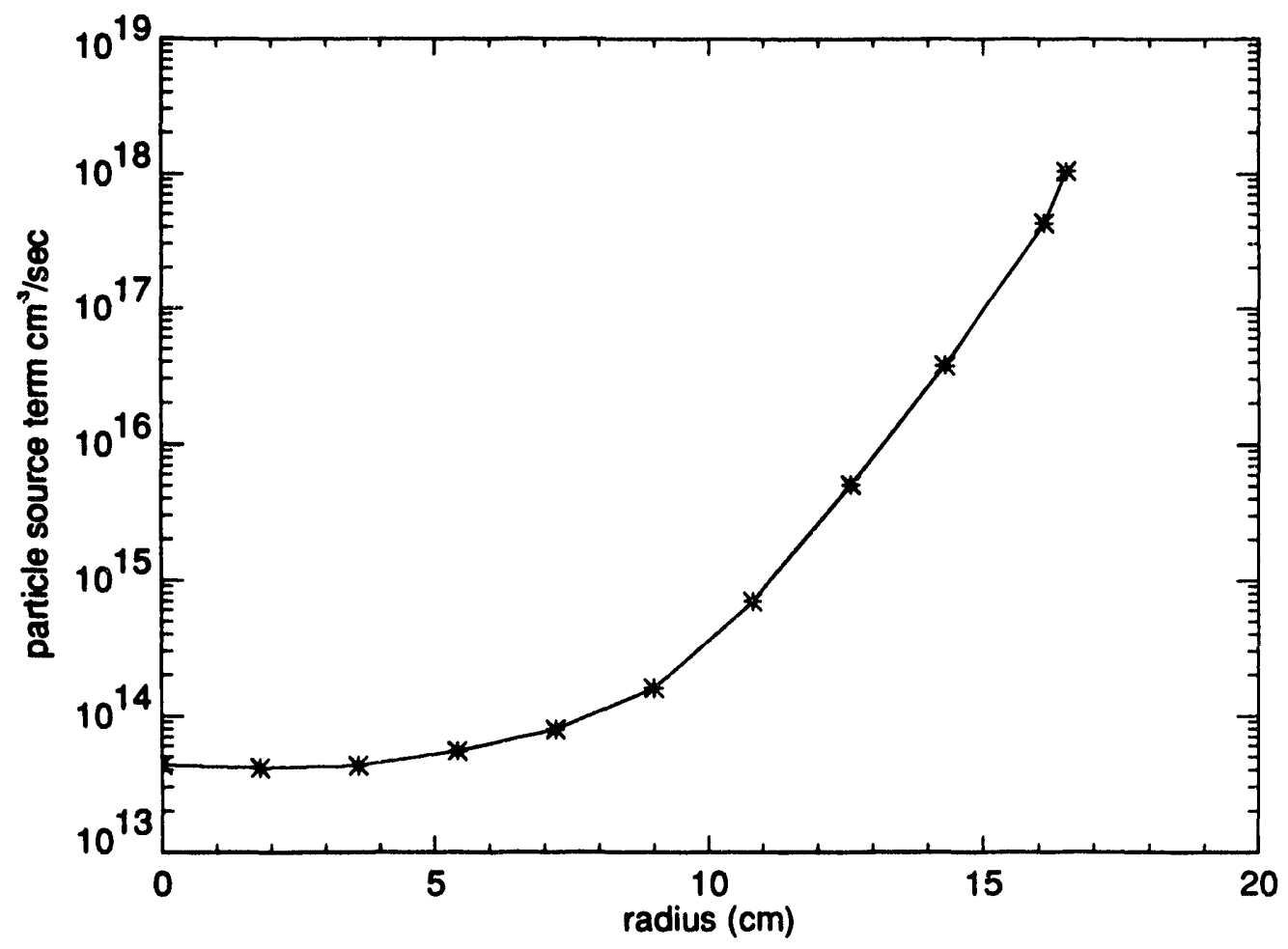

Figure 3.7: Profile of particle source term for shot 14079 at $280 \mathrm{msec}$ calculated from ONETWO.

The global particle confinement time for the steady state plasma is given by

$$
\tau_{p}=\frac{\int n d V}{\int \Gamma d S} .
$$

The above set of assumptions for relating scrape-off layer parameters to the flow rate is one of two proposed in reference [54]. The other set proposed yields values of the total flux at the plasma edge factor of 2 higher than equation 3.21. Thus estimates of the global particle confinement time given from equations 3.21 and 3.22 yield estimates of global particle confinement times a factor of two larger than the estimates, based on the former assumptions, presented in reference [54]. As an example the results of the analysis at a time during the steady state portion of gas modulation shot 14079 are presented in figure 3.7. In the ONETWO simulation the scrape-off layer is appropriately modelled by assigning it a width equal to the poloidal average of the measured decay length and using a temperature representative of the measured scrape-off layer values. For the relevant conditions these are $\lambda_{n}=0.4 \mathrm{~cm}$ and $\mathrm{Te}=$ $15 \mathrm{eV}$. A global particle confinement time of $3 \mathrm{msec}$ was used. The ion temperature 
profile was calculated assuming an ion thermal conductivity 5 times the neoclassical value. Values of the neoclassical multiplier are inferred by matching the neutron production rate. The particle source term calculated in the outer plasma is well represented as an exponential

$$
S=S_{a} e^{\frac{(\rho-1) a}{\xi_{n}}} \quad \rho \leq 1
$$

which decays rapidly from the edge. The fit to the value of $\xi_{n}$ here is $1.3 \mathrm{~cm}$. The parameter $\xi_{n}$ decreases with increasing density of the outer plasma.

The $\mathrm{H}_{\alpha}$ detector on MTX views the plasma through a chord along the plasma equator from the outside of the torus. Given the asymmetries in the edge plasma, including the localized high radiation densities of Marfes, this signal could be used for only a rather crude order of magnitude estimate of the total particle source term. Since the signal amplitude can be greatly affected by the degree to which a Marfe overlaps the chord, even the trends in the global energy confinement time cannot be determined from this signal. For the sake of completeness estimates of the global particle confinement times of $\sim 4-25 \mathrm{msec}$ are obtained for these modulation discharges with use of a calibration obtained with a Unisphere B source.

A routine was implemented which generated simulated data by solving the continuity equation with given profiles of $D(r), V(r)$ and with a source function

$$
S(r, t)=S_{o} e^{\left(\frac{(\rho-1) a}{\xi_{o}}\right)} G(t),
$$

where $\mathrm{G}(\mathrm{t})$ is specified for the simulation. Simulated results for $\tilde{n}(\mathrm{r}, \mathrm{t})$ could be input directly into the linear regression analysis routine to reproduce the analytic functions forms for $D(r), V(r)$ in regions where the source term was insignificant when no radial averaging was used. If we assume that the steady state and perturbed particle source terms have the same spatial profiles then we can examine the influence of the source term for various values of the source term decay length obtained from ONETWO simulation. An example is given for a value of $\xi_{s}=0.8 \mathrm{~cm}$ and

$$
\begin{aligned}
& V(\rho)=2 \rho+6 \rho^{2} \\
& D(\rho)=0.04+0.04 \rho+0.4 \rho^{2}
\end{aligned}
$$



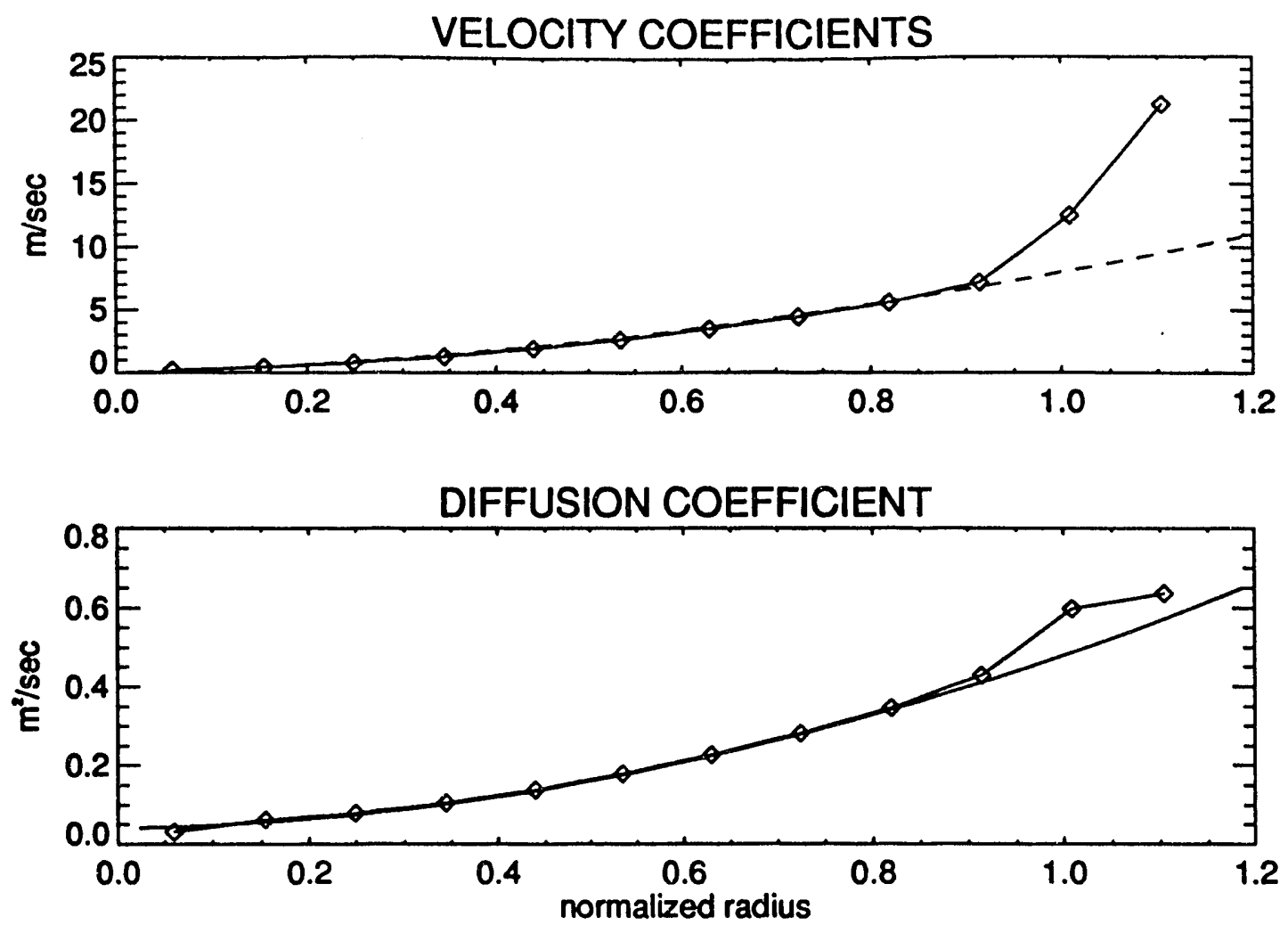

Figure 3.8: Comparison of results of analysis neglecting source terms with analytic results

which are representative values for the high-density shots considered. Perturbed density profiles calculated with this routine were analyzed directly via eq. 3.20 , in which the particle source term is absent. Results are compared with the analytic forms for $\mathrm{D}, \mathrm{V}$ in fig. 3.8. The neglected source term is not negligible for $\mathrm{r} / \mathrm{a}>0.8$ as expected. Since the integral equation 3.20 has an inner boundary condition at $r=0$, the particle source term, which is negligible except at the very edge of the plasma, has a negligible impact upon the results for $\mathrm{r} / \mathrm{a}<0.8$ and can be ignored there. This same result is in fact obtained for all of the values of $\xi_{n}$ obtained for shots in this study. Since the profile of the particle source term could not be measured directly and because particle transport in the edge plasma is asymmetric, only particle transport coefficients derived for the region $\mathrm{r} / \mathrm{a}<0.8$ will be considered.

The time varying density profiles calculated with the simulation routine were converted into equivalent line-integrated signals at the positions of the MTX chords. The analysis routine described earlier was applied to these signals yielding the results 


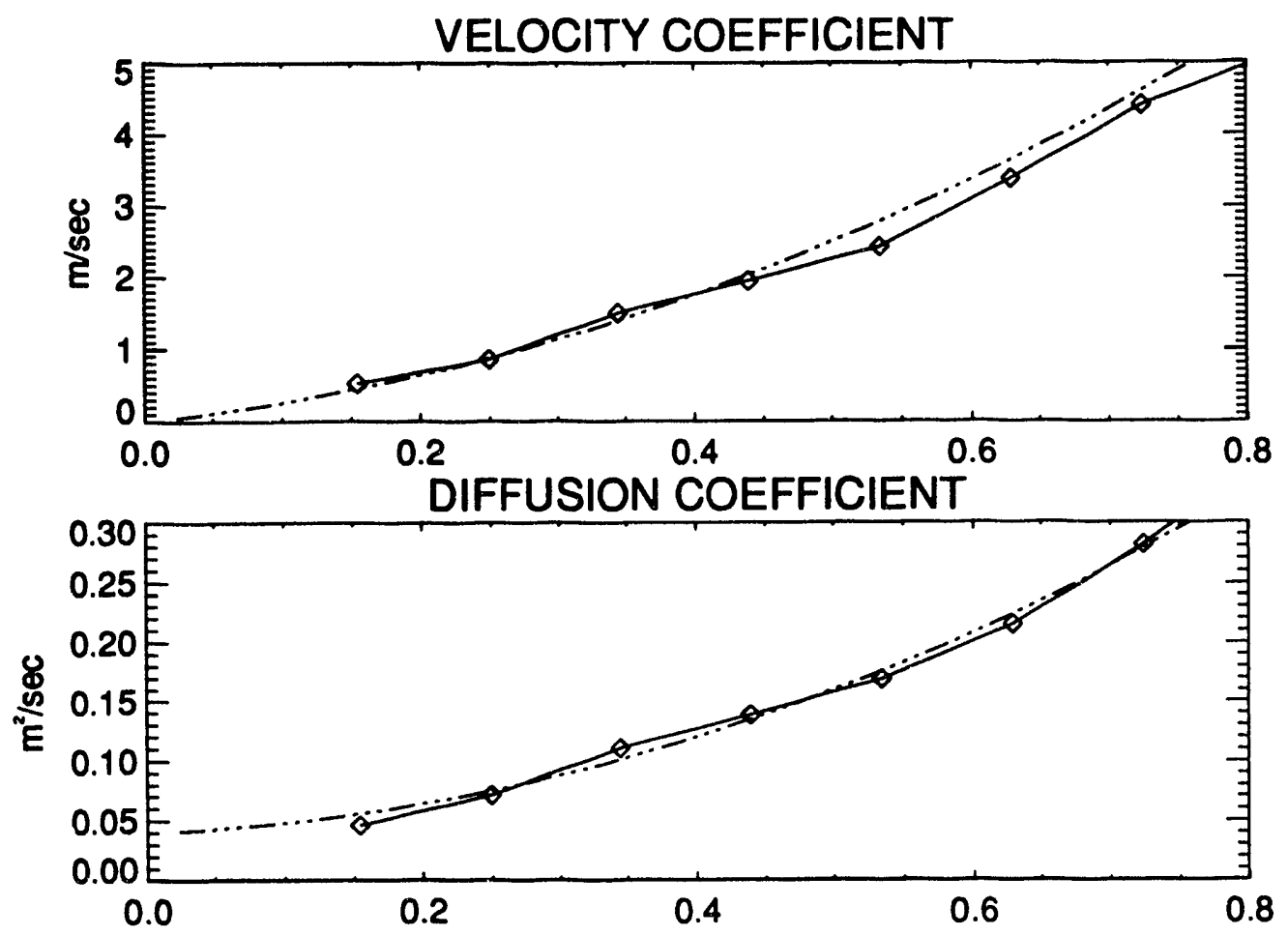

Figure 3.9: Comparison of results of analysis with data generated at MTX chord positions with analytic forms

which are compared with the analytic forms for $\mathrm{D}, \mathrm{V}$ in fig. 3.9. The majority of error in the result is due to uncertainties introduced from the spline fit. The errors are modest, $\sim 10 \%$ for $\mathrm{D}$ and $\sim 20 \%$ for $\mathrm{V}$, in the outer portions of the plasma. Since the absolute magnitude of the errors is similar across the profile the fractional uncertainties of the smallest coefficient values, at small $r$, are the largest. The same exercise was performed for 15 evenly spaced chords spanning the same range of major radii as the MTX chords. The results, shown in fig 3.10, show that more accurate results can be obtained when the chords are evenly spaced. As noted earlier the port ribs made it impossible to space the interferometer chords evenly on MTX. 

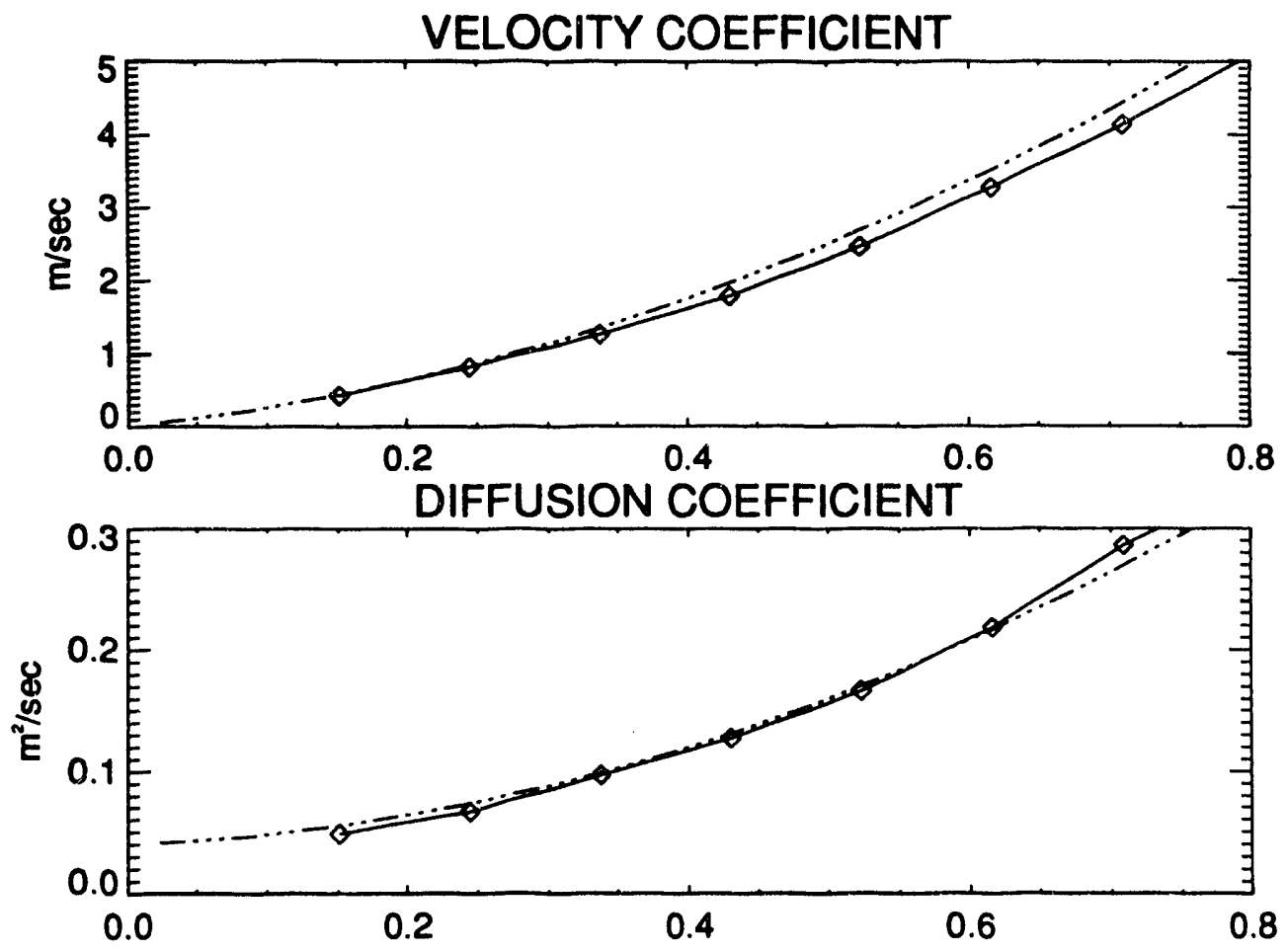

Figure 3.10: Comparison of results of analysis with data generated at evenly spaced positions with the analytic forms 


\subsection{Effect of Filtering and Influence of Spline Fit Parameters}

The application of the composite $60 \mathrm{~Hz} / 110 \mathrm{~Hz}$ band stop, $90 \mathrm{~Hz}$ low pass filter to the signal will tend to cause some distortion to the gas modulation data, but is essential because of the presence of periodic noise at $60 \mathrm{~Hz}$ and other discrete frequencies. The uncertainties in the results of the analysis are due to a number of factors such as uncertainties resulting from the spline fit, particularly with the gaps caused by the port ribs, calibration errors, noise in the chord signals and uncertainties in the position and geometry of the plasma.

When the analysis routine is applied to simulated data, the most accurate results are usually obtained without filtering and with $s=0$ in the spline routine so that the fit passes through the data points with no smoothing. Of course, accurate results are obtained only when the convective and diffusive portions of the flux can be distinguished and the perturbed flux is sizable all across the plasma column. This constrains the choices of $V(r), D(r)$ and the time scale of the perturbation $G(t)$ used in the simulation in the manner discussed in section 2.3. When these parameters are chosen so that the accuracy of the results obtained are reduced or when systematic errors, such as in the plasma position, are introduced smoother profiles of $\mathrm{D}(\mathrm{r})$, $\mathrm{V}(\mathrm{r})$, which are on the average more accurate, can often be obtained through the introduction of some smoothing into the spline fit $(0<\mathrm{s}<15)$. The values of the transport coefficients show essentially no dependence upon the choice of the spline fit parameters $\sigma$ in eq. 3.11 over the range $0 \leq \sigma \leq 1$. The value of sigma is set to 1 for all of the analysis presented here.

When significant smoothing is employed, the application of the low pass filter to the simulated data does not cause a substantial increase in the overall errors in $D(r), V(r)$. In some cases, when smoothing is used in the spline fitting routine, more accurate values are obtained for $V(r), D(r)$ derived from filtered simulated data than for unfiltered data.

The effect of the lowpass filtering was assesed on a simulated signal generated 


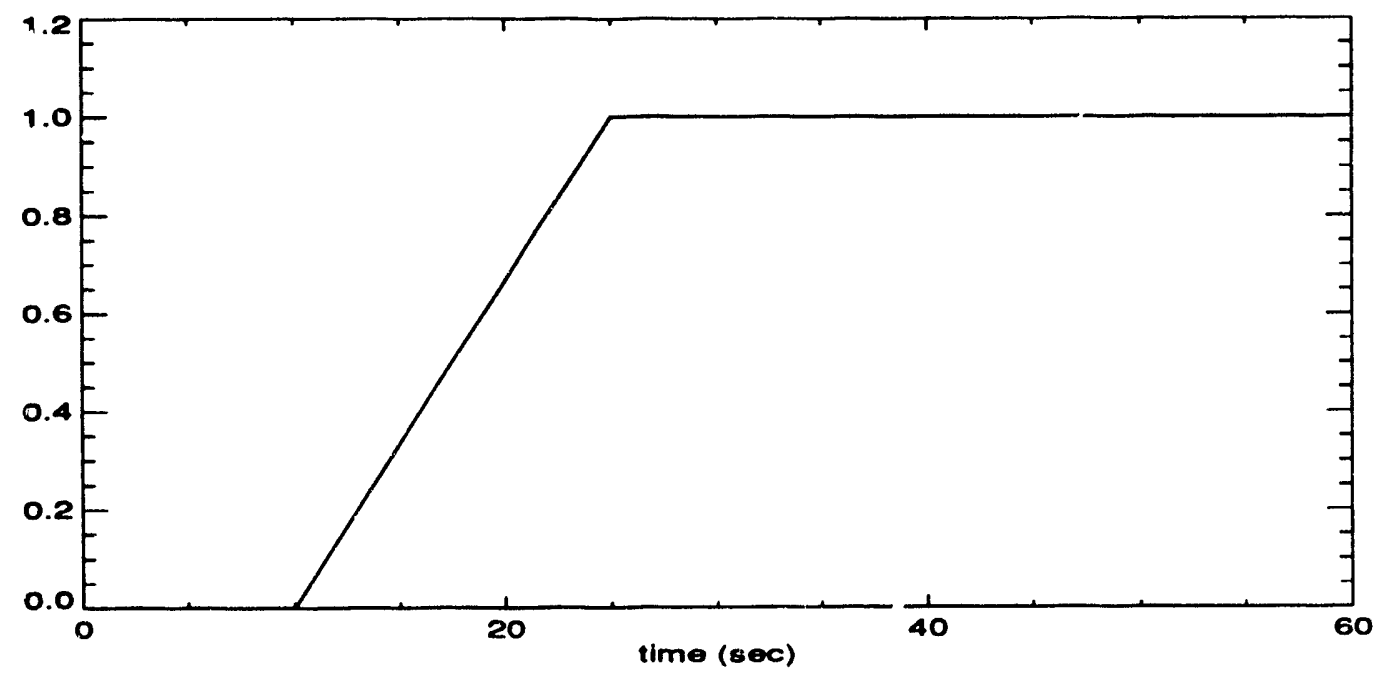

Figure 3.11: The function $G(t)$ used in numerical simulation in the time domain.

with this routine using the parameters

$$
\begin{aligned}
V(\rho) & =2 \rho+6 \rho^{2} \\
D(\rho) & =.04+.04 \rho+.8 \rho^{2} \\
S=S_{o} e^{-\frac{(\rho-1) a}{\xi_{n}}} G(t), & \xi_{n}=0.8 \mathrm{~cm}
\end{aligned}
$$

The function usod for $G(t)$ is illustrated in fig. 3.11. The signal calculated for the central chord was processed with the bandstop/lowpass filter and is compared with the unfiltered signal which is shown as a solid curve in fig 3.12. Besides the characteristic time delay, the filtering causes some noticeable distortion to the signal waveform. Despite this the overall errors in the coefficients $D(r), V(r)$ obtained from the analysis of the filtered, simulated data with $\mathrm{s}=0$, shown in fig. 3.13 , are not significantly worse than for the case without filtering shown in fig. 3.9. The bulk of the transport information in the signal is carried by the low frequency components which were not substantially distorted by the filter. For much, much larger values of transport coefficients the $60 \mathrm{~Hz}$ bandstop $/ 90 \mathrm{~Hz}$ lowpass filter could become a dominant source of error. As we shall see, however, the coefficient profiles used in the last simulation ha"e magnitudes which are representative of the values determined for MTX plasmas. The coefficient profiles obtained from the analysis of MTX data show no systematic trend as the low pass filter frequency is increased to much larger values, other than to 


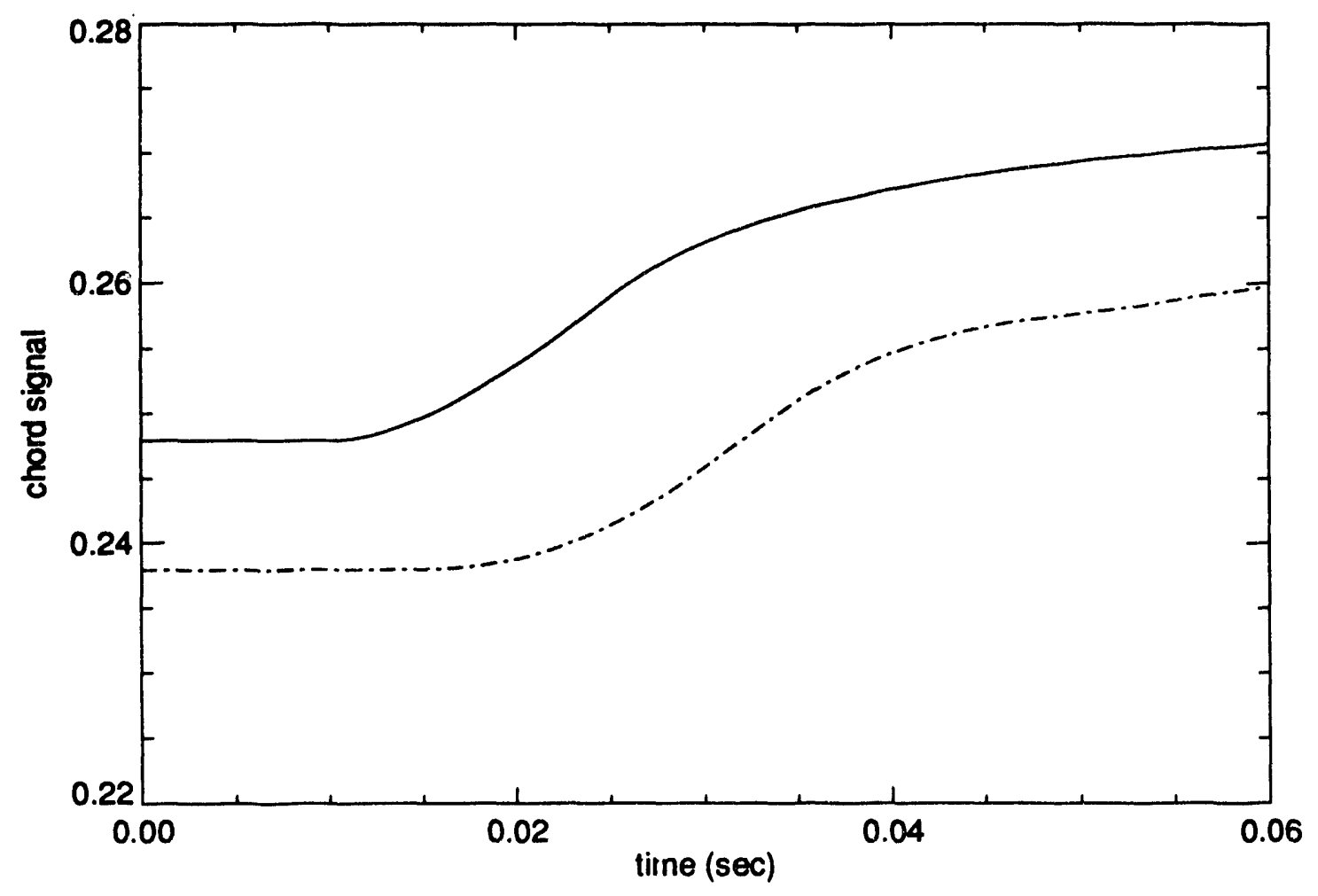

Figure 3.12: Comparison of simulated signal with and without filtering.
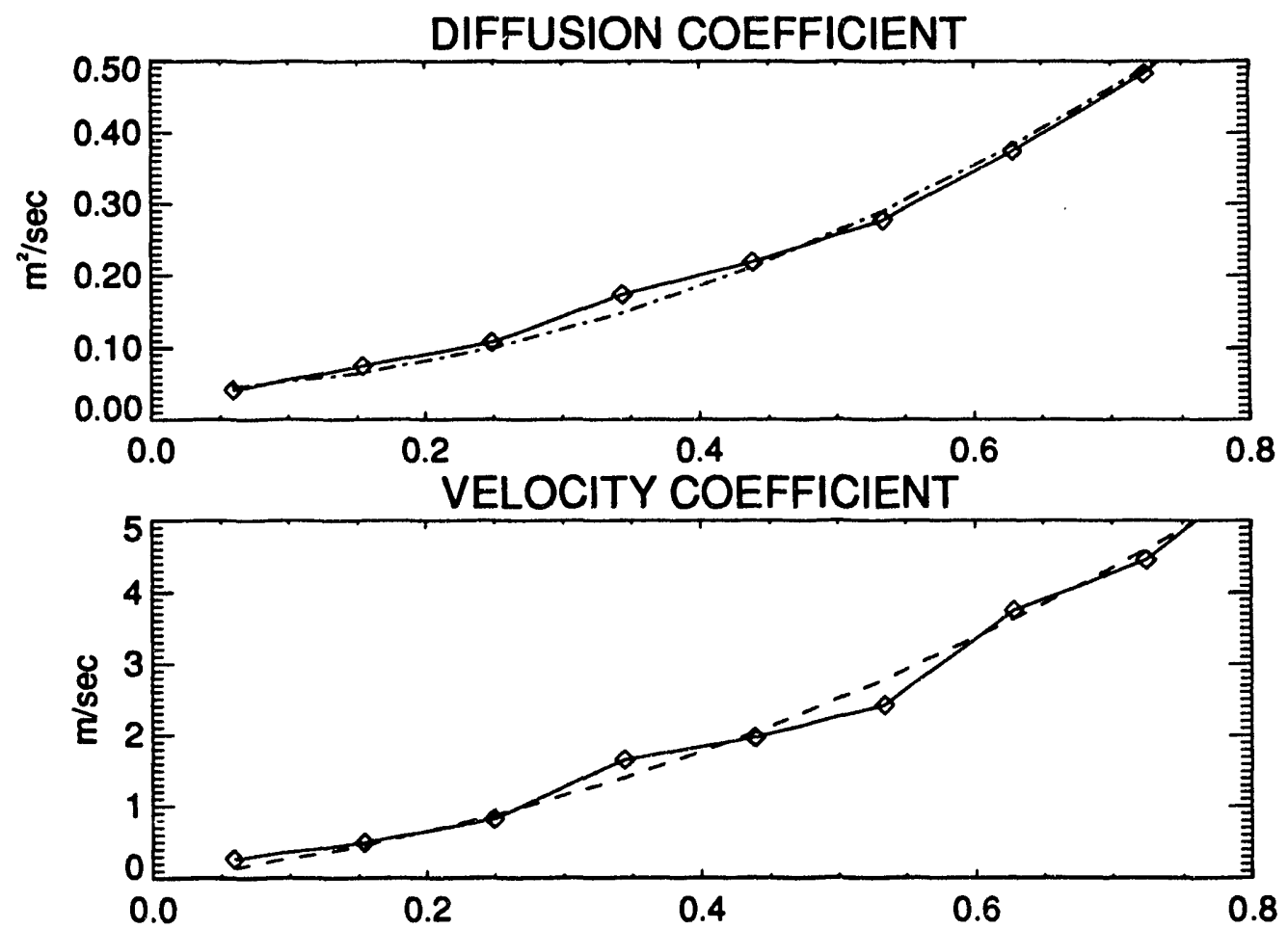

Figure 3.13: Transport coefficients obtained with filtering of simulated data. 
become more bumpy, due to the increased noise content of the signal. Thus compared with uther errors, such as those introduced through the spline fit, the distortion of the density modulation transient signal from the application of the bandstop/lowpass filter is an unimportant source of error. The principal limitations on the accuracy of the results obtained stem from the finite number of chord positions at which measurements are made ${ }_{r}$ and particularly the gaps caused by the port ribs, as well as systematic errors associated with uncertainties in plasma position and geometry.

\subsection{Spectral Analysis Methods}

When periodic gas modulation is used a spectral method of analysis is most appropriate. Usually the fundamental modulation frequency component of the signal is analyzed. Complex coefficients for the density are used in the spectral form of the contrnuity equation. Two such approaches are outlined in Appendix F. These resemble in several respects the approach outlined for the time domain in this chapter.

\subsection{Summary of Analysis Procedure}

The steps in the data analysis procedure can be summarized as

- 1. remove fringe shifts

- 2. apply bandstop/lowpass filter

- 3. determine background fit at reference time

- 4. Apply spectral or time domain analysis.

Results from several shots are summed. These algorithms are implemented in a code written in IDL 2 specifically for this application. 


\section{Chapter 4}

\section{Experimental Results}

Gas modulation experiments were conducted at a variety of line-averaged densities and plasma currents, all at a toroidal field of $6.25 \mathrm{~T}$. Triangular data points shown in fig 4.1 each consist of a shot, repeated 8-10 times, where a single puff density modulation occurs in which background plasma parameters are held nearly constant. Square data points represent shots with periodic density modulations which meet the same criterion for the background conditions. These were repeated a total of six times or less. Data from these discharges is discussed and analyzed in the following sections.

The potential impact of certain experimentally observed phenomena on the measurement must be addressed. It is shown in sec. 4.1 that the basis function fit routine discussed in the previous chapter can fit the chord data well with realistic input parameters. Edge asymmetries represent a potential source of difficulty for the analysis. Data from the DENSEPACK array and the MTX interferometer, discussed in sec. 4.2 , form very strong evidence that little change occurs in the asymmetric edge density during gas modulation in the high-density discharges, so that the impact of the asymmetries on the results of the analysis is minimal.

The behavior of the electron density, temperature and particle transport coefficient profiles and global energy confinement time as the density limit is approached are covered in sec. 4.3. Some differences were seen in density limit behavior at different values of plasma current, as discussed in the following section. 


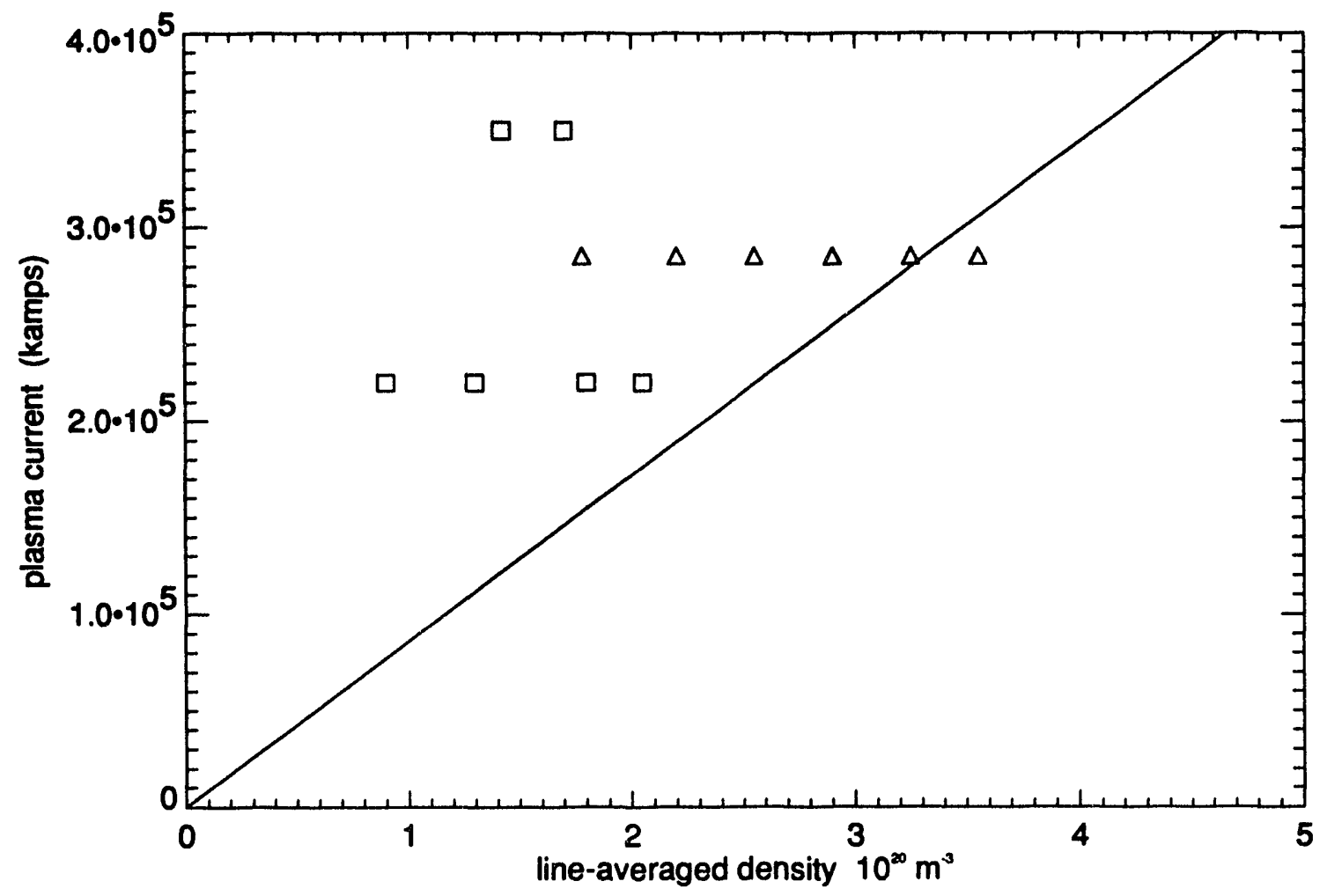

Figure 4.1: Line-averaged densities and currents at which gas modulation data was taken.

Results not directly related to the density limit are also given in this chapter. A comparison of the ratios of $\mathrm{aV} / \mathrm{D}$ to a/ $\mathrm{L}_{n}$ in sec. 4.5 reveals values similar to those expected from an equilibrium balance in the plasma core. The effect of off diagonal terms and coefficient dependences on plasma parameters is found to be significant. Particle transport coefficients are also compared with values of $\chi_{H P}$ determined earlier from sawtooth propagation. The existence of anomalous inward particle convection in MTX is demonstrated.

Discharges with periodic gas modulation are analyzed in sec. 4.7 and the results are compared with those from analysis in the time domain in sec. 4.8. The validity of the model for the particle flux used in the analysis is considered. It is shown that the model is a statistically meaningful description of the data. The effect of temperature perturbations is contained in the convection velocity $\mathrm{V}$. Uncertainties in these measured particle transport coefficients are estimated.

We begin by examining a typical fit to the density profile at a reference time. 


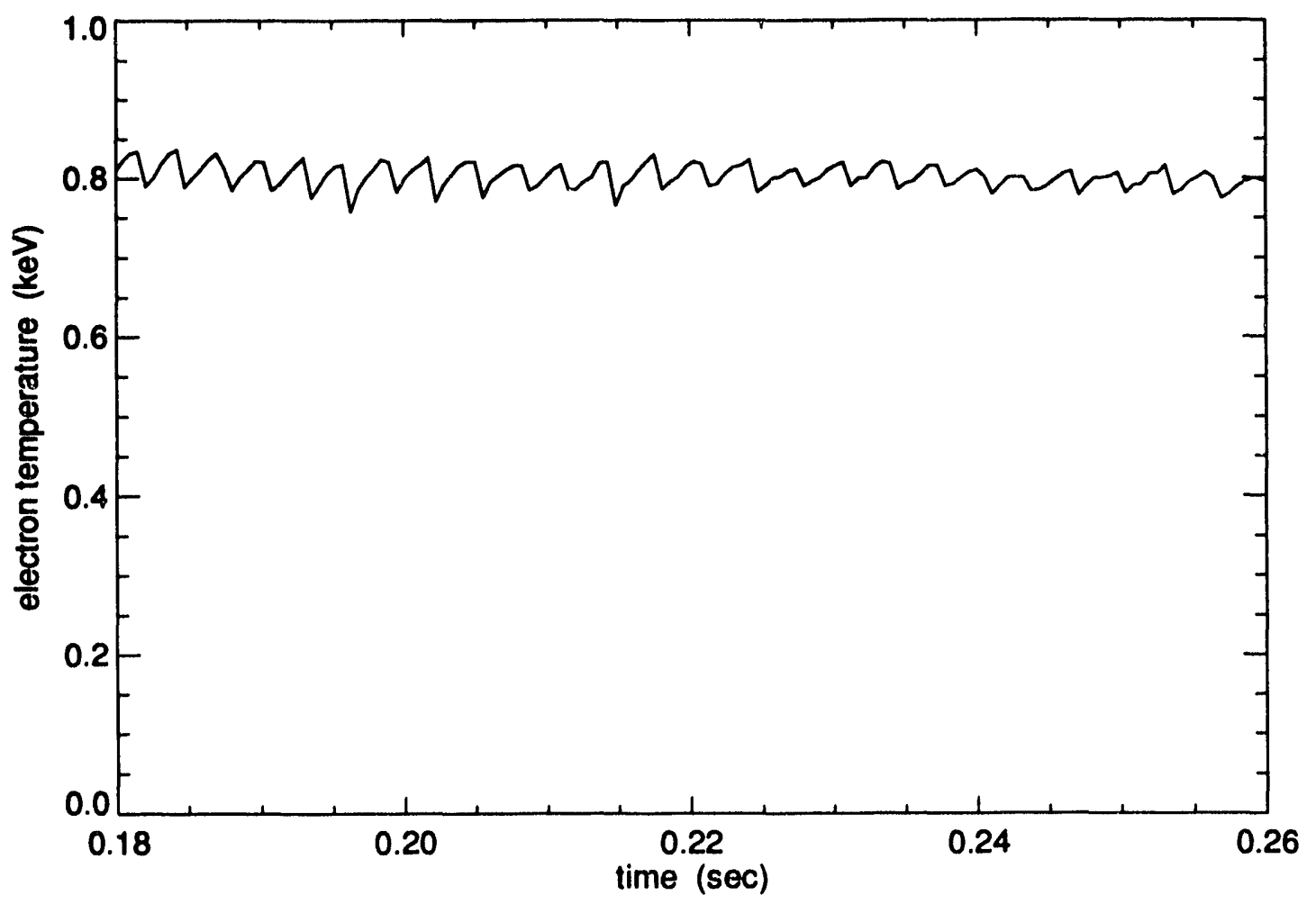

Figure 4.2: Weak sawteeth on central polychrometer channel, shot 14079.

\subsection{Density Profiles}

All of the shots represented in fig 4.1 were sawtoothing discharges. For the high $\mathrm{q}$ shots at 210 and $285 \mathrm{kA}$ the sawtooth amplitude on the central polychrometer channel is fairly weak, as shown in fig. 4.2 for shot 14079 with $\mathrm{q}_{a}=4.8$. The background density profile fit for shot $14108\left(\bar{n}_{e}=1.8 \times 10^{20} \mathrm{~m}^{-3}, \mathrm{I}_{p}=285 \mathrm{kA}\right)$ is shown in fig 4.3. The density profile is flat near the center, as is often the case for these sawtoothing discharges. Recall that the data has been lowpass filtered at $90 \mathrm{~Hz}$ so that this fit profile is averaged over the sawtooth period. The density at $\rho=1$, the last closed flux surface, is higher than that at the outside limiter. The separation between the two at the outside of the tokarnak is due to the slight inward offset of the plasma. The offset parameter determined by the fit routine is $\delta_{o}=-.063$ equal to $1.0 \mathrm{~cm}$. This is compared to results determined from a routine which uses polarimeter, interferometer and other data to fit the equilibrium geometry using the MHD equilibrium equations [55]. In that code the plasma position is determined essentially from po- 


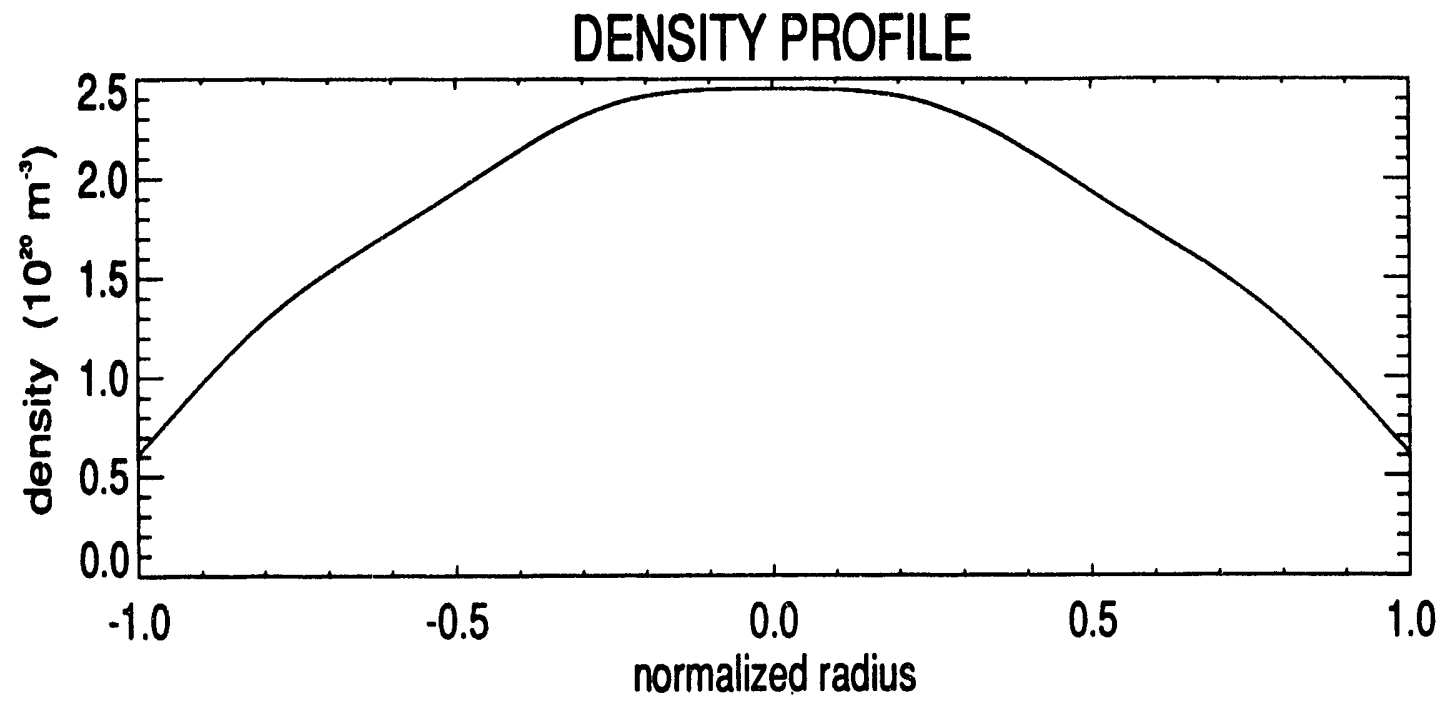

FIT TO CHORD SIGNALS

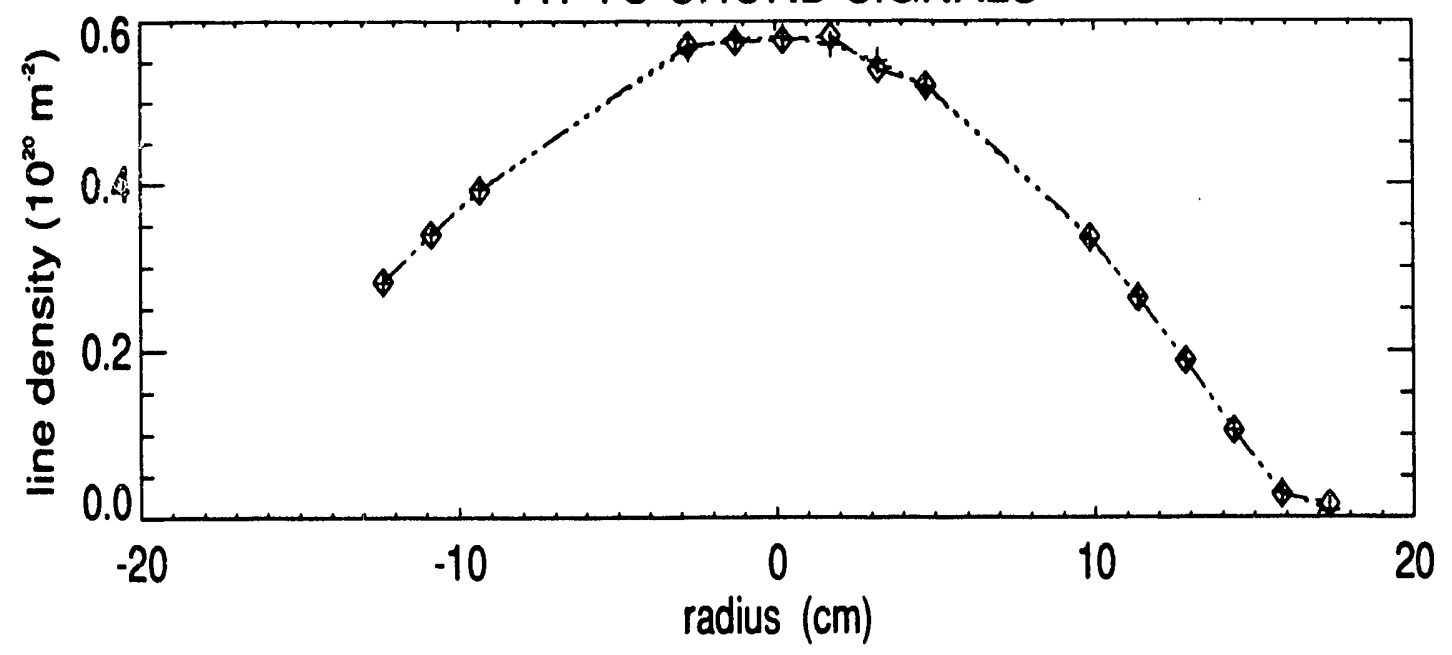

Figure 4.3: Background density profile and fit to chord data for shot 14108 . 
larimeter data. The offset it determined for shot 14108 is $4 \mathrm{~mm}$ different than from the fit to the interferometer data. This small discrepancy between the plasma offsets determined from polarimeter and interferometer data is present on many shots and was reported previously in a separate analysis by Rice [55]. It may be due to the poloidal asymmetries in the scrape-off layer (sol) density, uncertainties in the calculation of $\Delta(r)$ stemming from the inhomogeneous vertical field and uncertainties in the radial profile of $T_{i}$.

The Shafronov shift determined by this code exhibits very little sensitivity to plasma parameters over the range of currents and densities covered in the modulation experiments. Profiles of $\Delta(r)$ calculated by the code for a number of shots in the data base having rather different line-averaged densities and different values of current were well fit by $\Delta(\rho)=\epsilon_{o}\left(1-\rho^{\alpha}\right)$ with $\epsilon_{o} \simeq-.08$ and $\alpha=2.75$. Constant values of $\epsilon_{o}=-.0825, \alpha=2.75$, obtained for a particular shot, were used for the analysis of all of the gas modulation shot data.

\subsection{Edge Asymmetries and Measured Density Profile Evolution}

A local increase in the density scrape-off length can occur at the poloidal location of the Marfe, accompanied by a decrease at other poloidal locations. LaBombard [54] noted that the Marfes were detected, however, as relatively small perturbations to the already asymmetric scrape-off layer density. The location of the Marfes determined from probe measurements coincides with that from spectroscopic measurements [54]. In the shots used for determinations of particle transport coefficients here, indication of Marfe activity on the visible bremsstrahlung and FIR interferometer were present only on the inner chords. The three innermost interferometer chords were excluded from the analysis of Marfeing shots. There was no indication of a Marfe on the other 12 interferometer chords during modulation, nor on the visible bremsstrahlung chords in the same region. As noted on page 40, DENSEPACK measurements indicate that there is only a rather slight increase in normalized poloidal edge density asymme- 


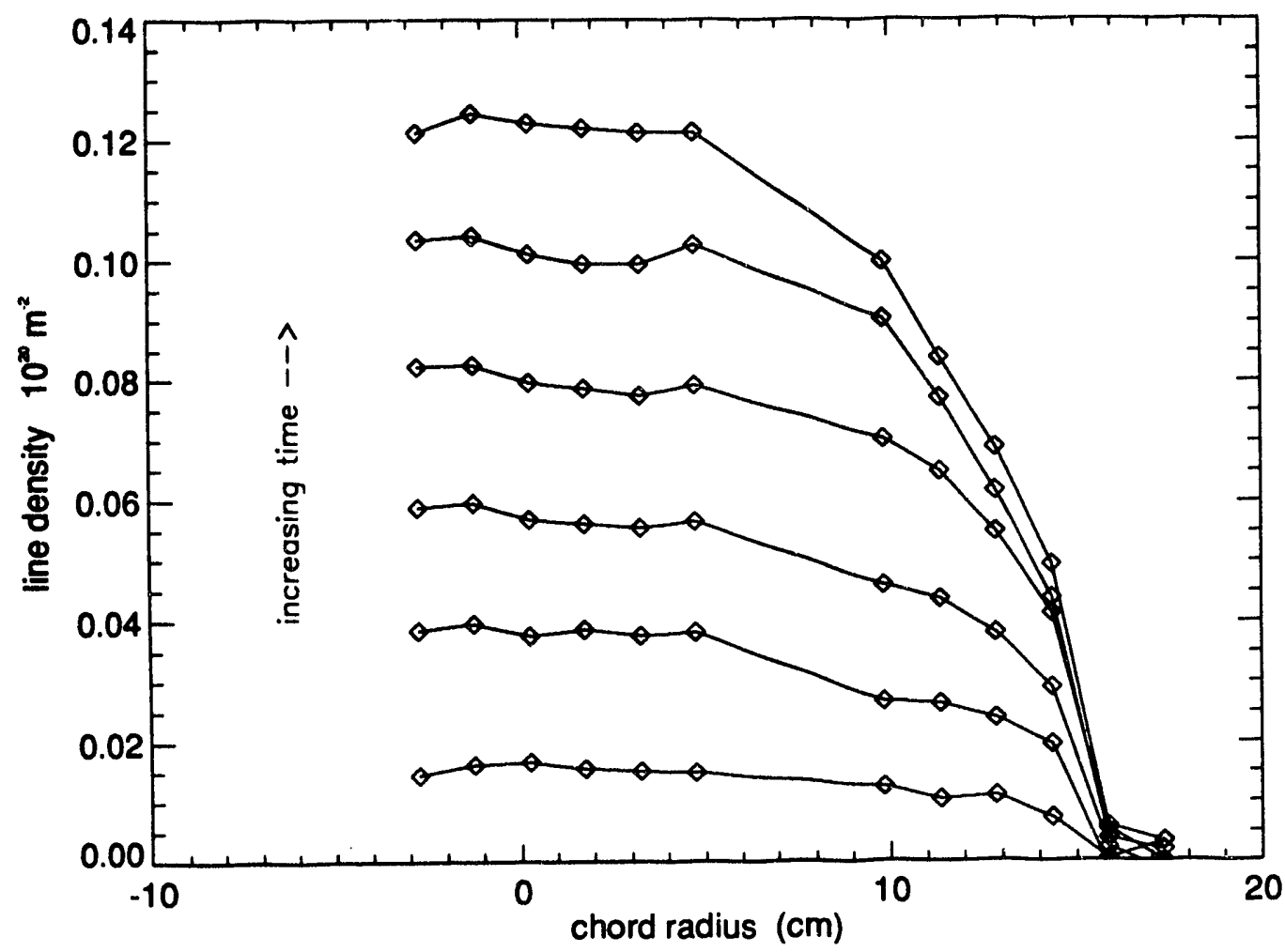

Figure 4.4: Change in FIR chord signals following a gas puff for $\bar{n}_{e}=3.3 \times 10^{20} \mathrm{~m}^{-3}$, $\mathrm{I}_{p}=285 \mathrm{kA}$.

tries at high core density values [54]. The angular positions of the density extrema remain the same for any value of $\bar{n}_{e} / I_{p}$. The normalized magnitudes and positions of the density extrema are insensitive to the presence of Marfes. Edge asymmetries contribute to the modulation signal only to the extent the plasma density at the edge changes during modulation.

The change in FIR chord signals following a single gas puff is shown at various increasing time intervals in fig. 4.4. The two outermost chords show very little variation following the gas puff. This behavior is observed on all of the high-density gas modulation shots. With the effect of plasma refraction included, the second to last chord intersects the plasma equator at a position just inside the last closed flux surface. The behavior of these two outermost chords indicates that the change in the plasma density outside the last closed flux surface is quite small compared to changes occurring in the bulk plasma during modulation. Concurrently, inspection of fig. 4.4 reveals no indication of significant asymmetries in the change in the chord signals 


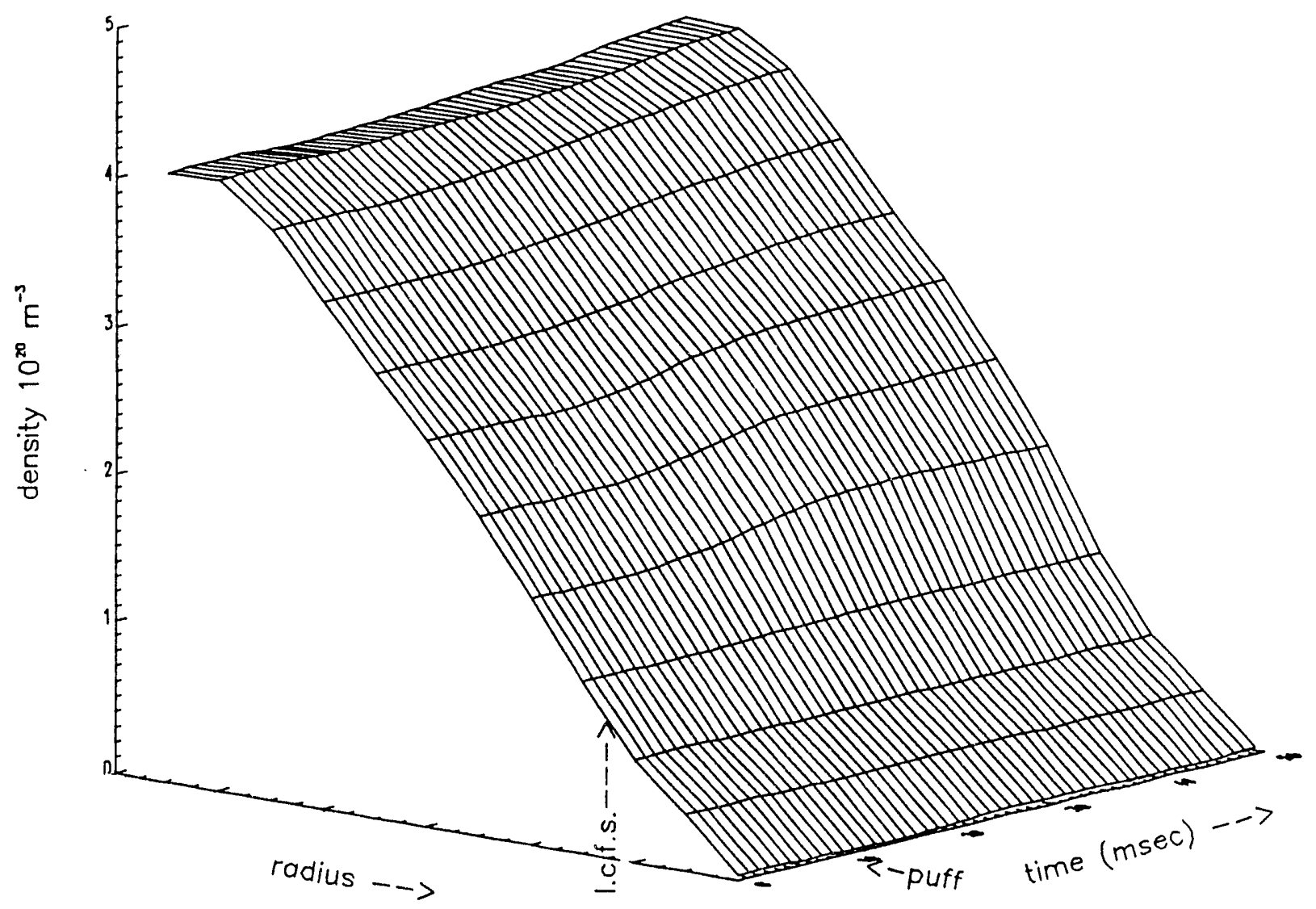

Figure 4.5: Evolution of density profile vs. flux surface radius and time during a gas puff.

following the puff. The change in the signals becomes more peaked at the center as the density wave propagates toward the center of the plasma. The shot examined here, at $\bar{n}_{e}=3.3 \times 10^{20} \mathrm{~m}^{-3}, \mathrm{I}_{p}=285 \mathrm{kA}$, is at the Greenwald density limit threshold $\left(\bar{n}_{e}=\kappa \bar{J}\right)$ and was the highest density at which useful modulation data was obtained.

The evolution of the density profile $\tilde{n}_{e}(\rho, t)$ is obtained through analysis in the time domain by "inverting" chord data at $1 \mathrm{msec}$ intervals as described in the previous chapter. In figure 4.5 a surface plot of the evolution of the density profile is given for background plasma conditions of $\bar{n}_{e}=2.2, \mathrm{I}_{p}=285 \mathrm{kA}$. Once again we see little change outside of the last closed flux surface during the puff, which occurs at around $10 \mathrm{msec}$ into the $50 \mathrm{msec}$ shown in the plot. The density rises rapidly in the outer regions of the plasma, but evolves on a slower time scale near the center of the plasma. This is characteristic of the evolution of the density perturbation observed 


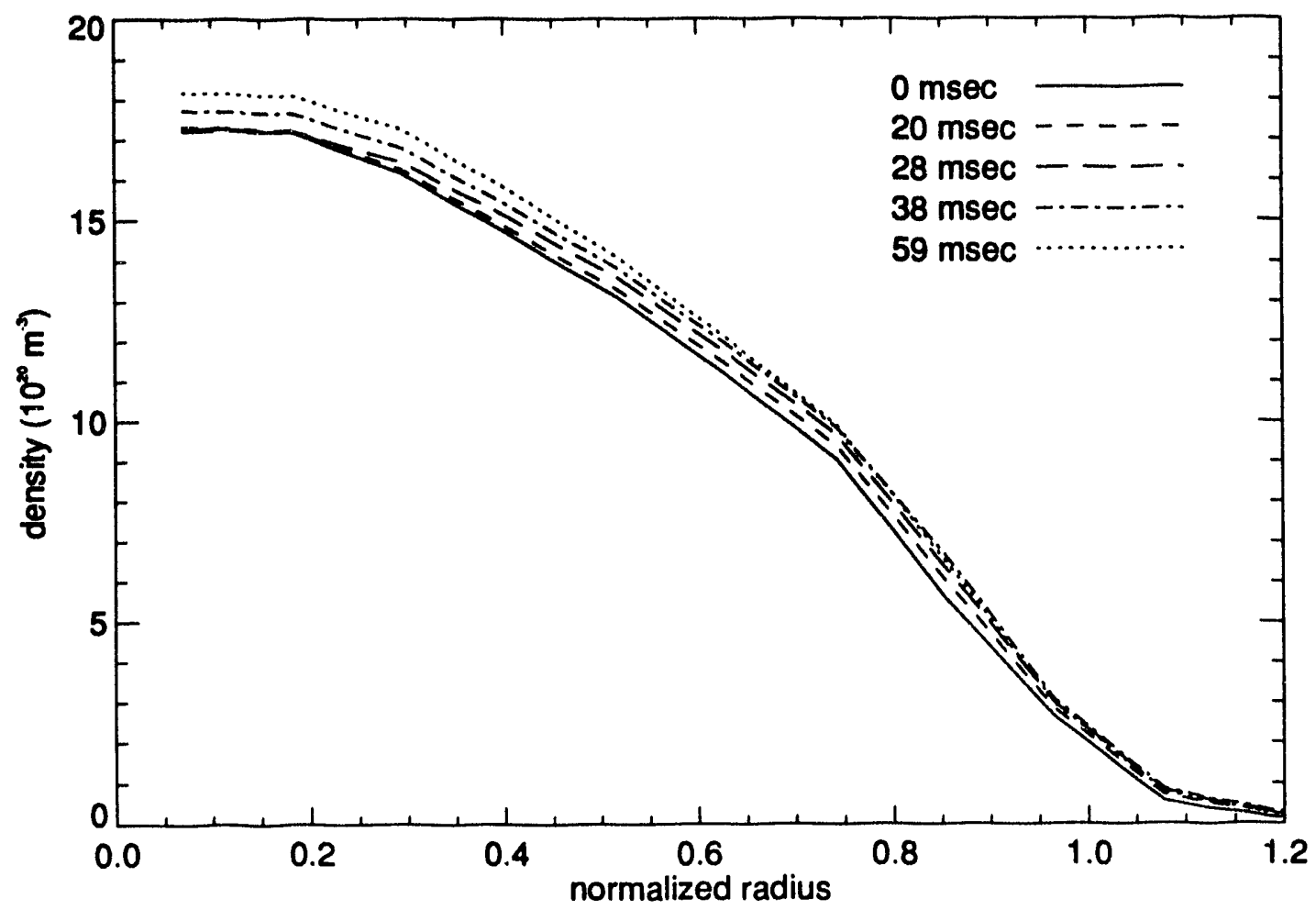

Figure 4.6: Evolution of the density profile at several times steps following the gas puff, $\bar{n}_{e}=1.8, \mathrm{I}_{p}=285 \mathrm{kA}$.

in high-density MTX plasmas. Profiles at several times are superimposed in fig. 4.6.

The length of the time period used for linear regression analysis is another important ronsideration. This is chosen so as to encompass the most significant portion of the transient induced by the gas puff, during which the perturbed flux is largest. The time window illustrated in fig. 4.5 is typical of that used for the analysis. The regression is applied to data starting at $\sim 10 \mathrm{msec}$ before the gas puff and continues for a total length of 50-70 msec. Beyond this the noise in the signals becomes more prominent than the perturbed flux which continues to diminish as the transient dies out. If the time window for regression analysis is extended for 10's of msec in either direction the additional time segments contribute mostly noise to the record and the overall signal to noise ratio for the record decreases. The profiles of transport coefficients show little change in their overall shape for such variations in the length of the record analyzed. If the amplitude of the noise contained in the additional segment is small compared to the flux from the modulation transient, the coefficient values 
show virtually no change due to the addition of the segment. If the noise in the added segment is substantial the resulting profiles of transport coefficients will tend to become more bumpy while retaining the same overall appearance. When the time span of the analysis is made too long, the overall change in the plasma parameters can become too great and the linearized analysis is no longer applicable. The time span of the record used for analysis is optimized to cover most of the variation of the plasma parameters during the transient, while maximizing the overall signal-to-noise ratio.

\subsection{Trends Observed as Density Limit is Ap- proached}

Six gas modulation data points, consisting of 8-10 shots each, were collected at $\mathrm{I}_{p}=285$ $\mathrm{kA}$ with average reference line densities of $\bar{n}_{e, 20}=1.8,2.2,2.6,2.9,3.3,3.6$ at the start of the gas puff. The highest line-averaged density obtained at this current, late in the shot just before rampdown of the $\mathrm{OH} 2$ coil was initiated, was $4.0 \times 10^{20} \mathrm{~m}^{-3}$. This is a full $20 \%$ above the limit scaling of Greenwald, who pointed out that it was only an approximate scaling law. Gas fuelled plasmas with values of $\bar{n}_{e} / \kappa \bar{J}$ of up to 1.2 were reported for Alcator $\mathrm{C}[16]$. The line-averaged central densities were increased by $8-12 \%$ by the gas puff for these shots. Densities at which data points were taken were closely spaced.

No substantial MHD activity was observed on any of the gas modulation shots during the quasi steady state portion of the discharge. This is so even though the highest densities at $285 \mathrm{kA}, 6.5 \mathrm{~T}$ are predicted to be in the vicinity of the Granetz threshold [81]. In deuterium discharges the Granetz threshold densities for the onset of MHD activity reported for Alcator C operations at MIT had substantial scatter, with a standard deviation of $\pm 20 \%$ [81]. On MTX study of data from a large number of shots indicates that the scatter in the amplitude of MHD activity increases with plasma current and density [82] in the vicinity of the Granetz threshold. But even in the vicinity of the Granetz threshold, the majority of those plasmas exhibit rather 


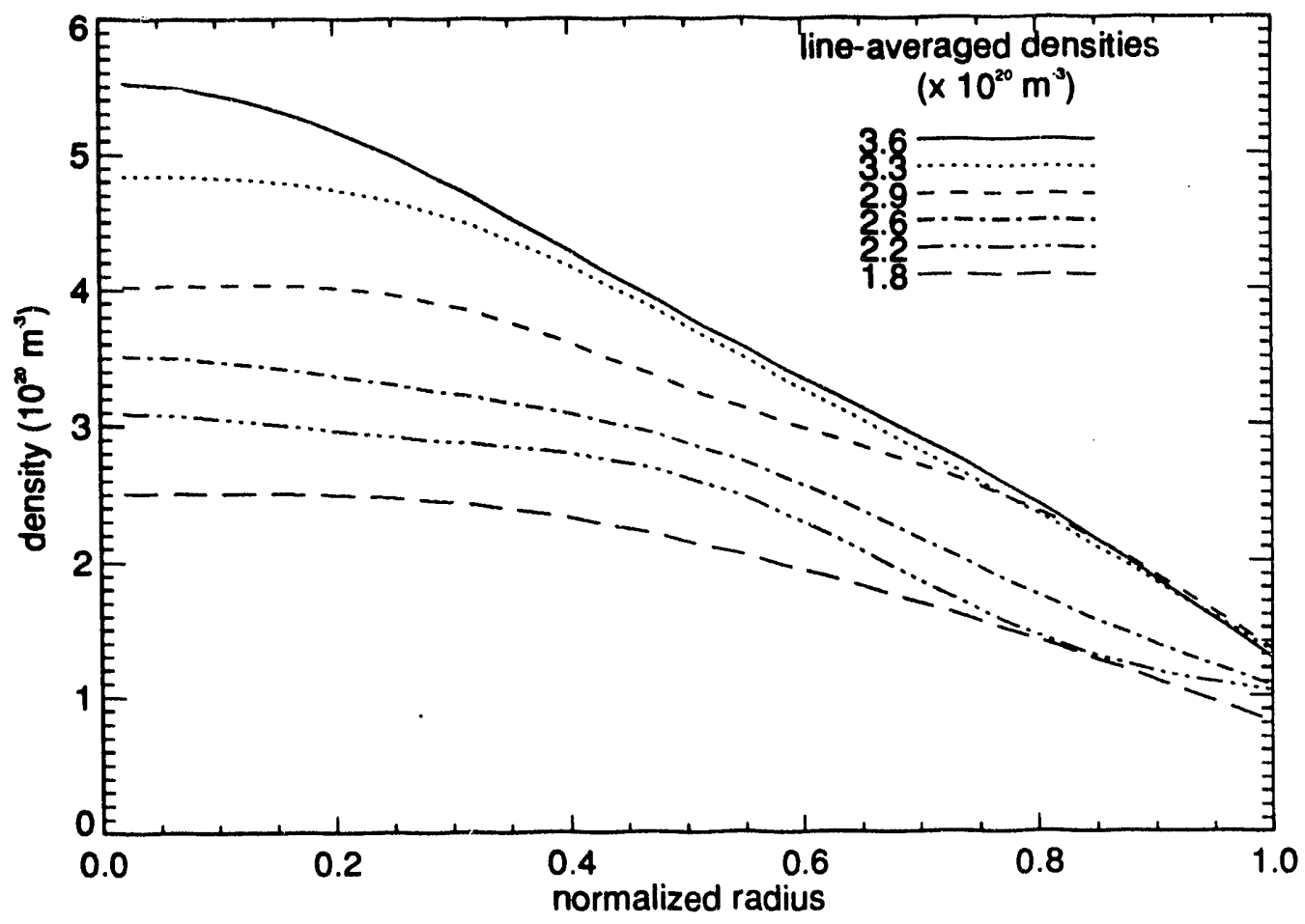

Figure 4.7: Density profiles obtained from basis function fits to $285 \mathrm{kA}$ data at 280 msec.

small amplitude MHD activity with little dependence upon current or density.

The background density profiles, obtained from basis function fits for the plasmas of the $285 \mathrm{kA}$ density scan at $260 \mathrm{msec}$, are shown in fig. 4.7 as a function of flux surface radius normalized to the last closed flux surface. To first order the profiles have similar shapes across the range of the scan. As the density limit is approached there appears to be less increase in the edge density in this data set. The increase in density is more prominent in the central region of the plasma. Fig. 4.8 shows profiles determined from the same data using the matrix inversion routine, which treats the regions inside and outside of the last closed flux surface in the same way. The interferometer data shows little change in the density in the outer plasma, especially outside of the last closed flux surface, as the line-averaged density is increased toward the limit.

For gas fuelled Alcator C plasmas it was reported that Marfes began to appear when the parameter $\bar{n}_{e} / \bar{J}$ reached $0.55 \pm .05$ [63]. For the plasma current of $285 \mathrm{kA}$, 


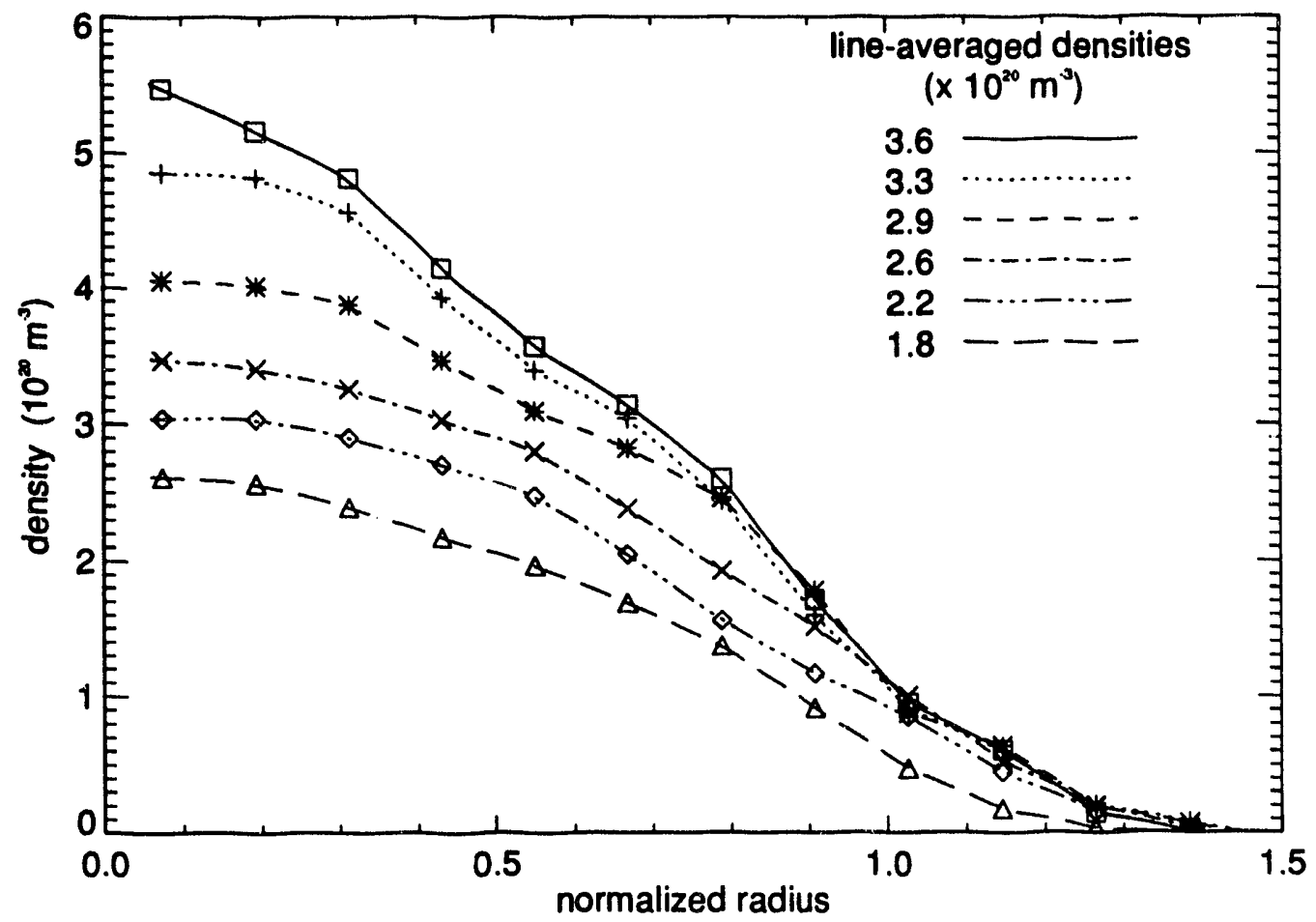

Figure 4.8: Density profiles obtained from matrix inversion of $285 \mathrm{kA}$ data at 280 msec.

this corresponds to a line-averaged density of $\bar{n}_{e, 20}=1.8 \pm .16$ for the Marfe threshold. The bolometer array could not be installed on MTX during the gas modulation run for lack of a sufficient number of available ports. Marfes could be detected through breakup of the inner interferometer chords as well as their dominant contribution to the innermost visible bremstrahllung chord signal. For example, radiation from a Marfe is quite conspicuous on the innermost visible bremstrahllung chord, at $\mathrm{r}=$ $-12.6 \mathrm{~cm}$ relative to the machine axis major radius, from $147 \mathrm{msec}$ to $240 \mathrm{msec}$ for shot 14038 shown in fig 4.9. Marfe activity is not apparent on the $285 \mathrm{kA}$ shots at $\bar{n}_{e, 20}=1.8$ except until very late in the shot, after the $\mathrm{OH}$ coil has begun its final rampdown. Marfes are clearly present on all of the $285 \mathrm{kA}$ shots at higher density, consistent with the scaling reported by the Alcator $\mathrm{C}$ group.

For line-averaged plasma densities below the Marfe threshold $\left(\bar{n}_{e} / \bar{J}<0.55\right)$ DENSEPACK probe measurements in the scrape-off layer indicate the poloidally averaged boundary density is strongly dependent upon the parameter $\bar{n}_{e} / \bar{J}[54]$. The 


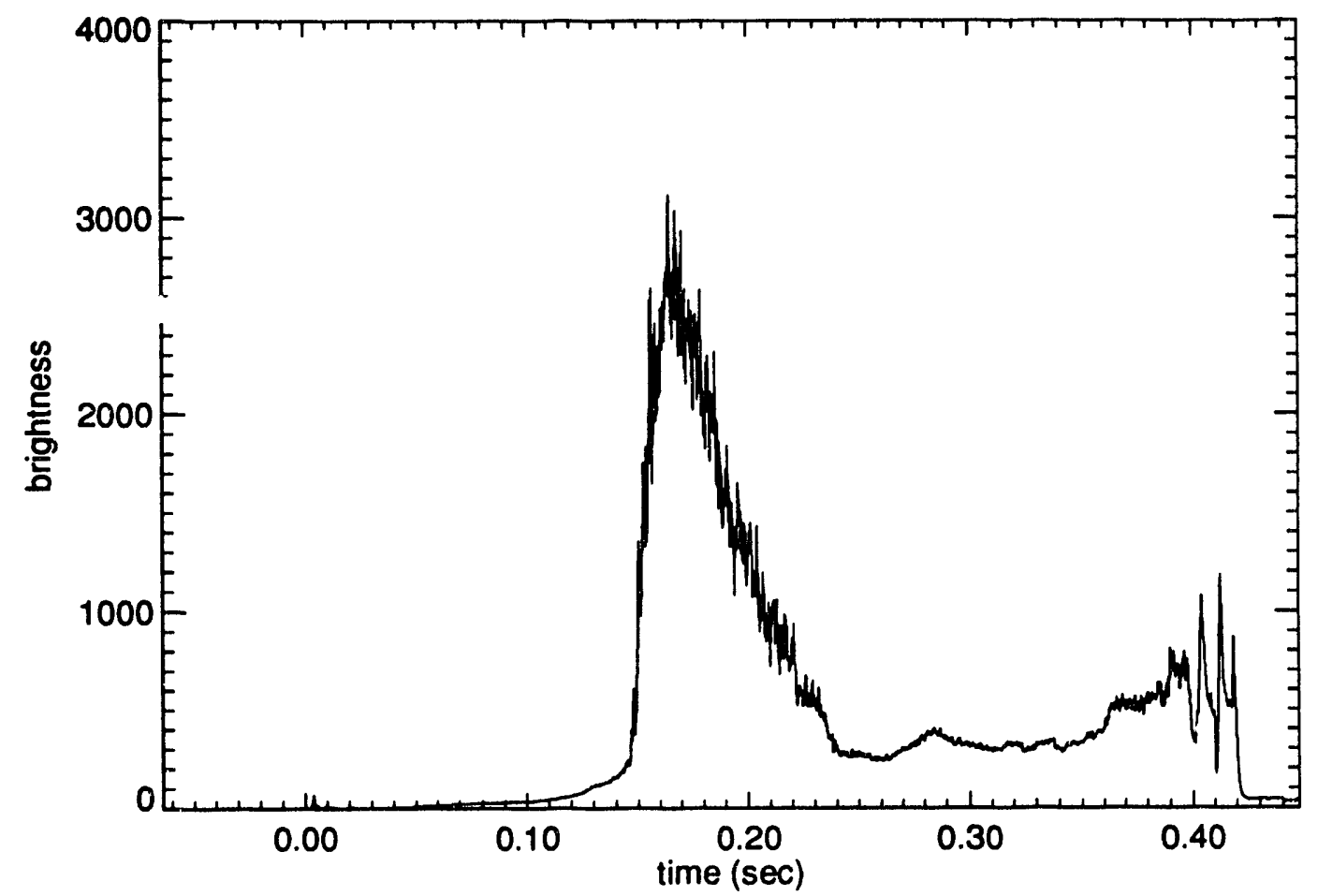

Figure 4.9: Marfe on visible bremstrahllung chord at $\mathrm{r}=-12.6 \mathrm{~cm}$ for shot 14038 with $\bar{n}_{e, 20}=2.9$ and $\mathrm{I}_{p}=285 \mathrm{kA}$. 


\begin{tabular}{|cccc|}
\hline $\mathrm{n}_{e, 20}$ & Bessel fit $(\mathrm{cm})$ & polarimeter mag. axis $(\mathrm{cm})$ & magnetics $(\mathrm{cm})$ \\
\hline 1.8 & 0 & 0 & 0 \\
2.2 & +0.22 & +0.1 & +0.2 \\
2.6 & +0.29 & +0.25 & +0.25 \\
2.9 & +0.25 & +0.3 & +0.3 \\
3.3 & +0.06 & +0.05 & +0.0 \\
\hline
\end{tabular}

Table 4.1: Plasma Position Relative to Value for $n_{e, 20}=1.8$

data could be well fit by a function of the form

$$
<n_{e b}>=\bar{n}_{e}\left(a+b\left(\frac{\bar{n}}{I_{p}}\right)^{\alpha}\right)
$$

with $\alpha=4 \pm 1$ and $\bar{n}_{e} / I_{p}$ in units of $10^{12} / \mathrm{cm}^{3}-k A$. In the region where Marfes are present $\left(\bar{n}_{e} / \bar{J}>0.55\right)$ the boundary density no longer shows a clear dependence upon the parameter $\bar{n}_{e} / \bar{J}$. In this regime the poloidal average of the boundary density is relatively insensitive to the central $\bar{n}_{e}$, but is sensitive to $l_{p}$, decreasing with increasing plasma current [54]. This is consistent with the trend in the background density profiles obtained for the shots from the density scan at the fixed current of $285 \mathrm{kA}$, shown in fig. 4.8. On all of the shots where Marfes are present $\left(\bar{n}_{e} \geq 2.2\right)$ the edge density profiles are similar, particularly outside of the last closed flux surface. This is also consistent with the observation that the scrape-off layer density changes very little during the gas puff on the high-density shots while the current is held constant.

The changes in average plasma position relative to the average values for $n_{e, 20}$ $=1.8$ were determined from three independent measurements. The Bessel function fit to the interferometer data is compared with the relative change in position of the magnetic axis determined from the "zero crossing" location from the polarimeter signals as well as the change in position measured with magnetic feedback loops. These are reported in Table 4.1 at the time of the gas puff. It is clear that through the $285 \mathrm{kA}$ density scan the horizontal plasma position is nearly constant. The offset from the Bessel function background fit changes in a manner similar to that from the polarimeter and the magnetic coils. The relative horizontal positions typically agree 
to within a fraction of a millimeter.

\subsubsection{Electron Temperature Profile Measurement}

A scanning Michelson Interferometer measures the spectrum of x-mode microwave radiation at the outside of the tokamak equator over the range from 100 to $730 \mathrm{Ghz}$ every $15 \mathrm{msec}$. The optical depth for single pass absorption of $\mathrm{x}$-mode radiation propagating normal to the magnetic field was given by Engelmann and Curatolo [60] for a plasma with a Maxwellian velocity distribution as

$$
\left.\tau\right|_{s=\frac{\pi}{2}}=\frac{\pi}{2} \frac{\omega_{p e}^{2}}{\Omega_{e} c} \frac{n^{2 n-2}}{(n-1) !}\left(\frac{k T_{e}(\bar{s})}{2 m_{e} c^{2}}\right)^{n-1} \frac{B_{o}(\bar{s})}{\left|\left(\frac{d B_{o}}{d s}\right)_{\bar{s}}\right|} .
$$

Here $\mathrm{n}$ is the harmonic number of the radiation, $\mathrm{s}$ is the angle between the ray and field line and $\tau \equiv \int \alpha d s$.

A theoretical expression for the $x$-mode radiation intensity derived considering the effect of multiple reflections from the tokamak wall is [61]

$$
I_{x}=I_{b} \frac{1-e^{-\tau}}{1-\hat{r} e^{-\tau}}
$$

where $\mathrm{I}_{B}=\omega^{2} k T_{e} / 8 \pi^{3} c^{2}$ is the black body radiation intensity and $\hat{r}$ is the reflection coefficient of the tokamak wall. According to this formula the plasma is optically thick if $\tau>>1-\hat{r}$. For stainless steel $1-\hat{r} \leq 10^{-2}$ for the frequencies in the range of interest for MTX. At the center of the plasma $\tau \geq 5$ for the second harmonic. The plasma is definitely optically thick there. Consider conditions near $\mathrm{r} / \mathrm{a}=0.8$ where, for these shots, we take as an example $\mathrm{T}_{e}=60 \mathrm{eV}, \bar{n}_{e}=2 \times 10^{20} \mathrm{~m}^{-3}$. Here $\tau=0.44 \gg 1-\hat{r}$ so that through the effect of multiple reflections from the walls we can expect the $\mathrm{x}$-mode radiation intensity at the frequency corresponding to this region to approach the black body value. At $\mathrm{r} / \mathrm{a} \sim 0.67$ where $\mathrm{T}_{e} \sim 200 \mathrm{eV}$, the optical depth for the same electron density is $\tau=1.5$. The ece Michelson microwave optics are located on a limiter port, so that the diagnostic view cannot focus back upon itself, but instead views rays which pass through the plasma at least several times. These may originate from regions having the same toroidal field strength, but off of the plasma equator, corresponding to colder plasma. 


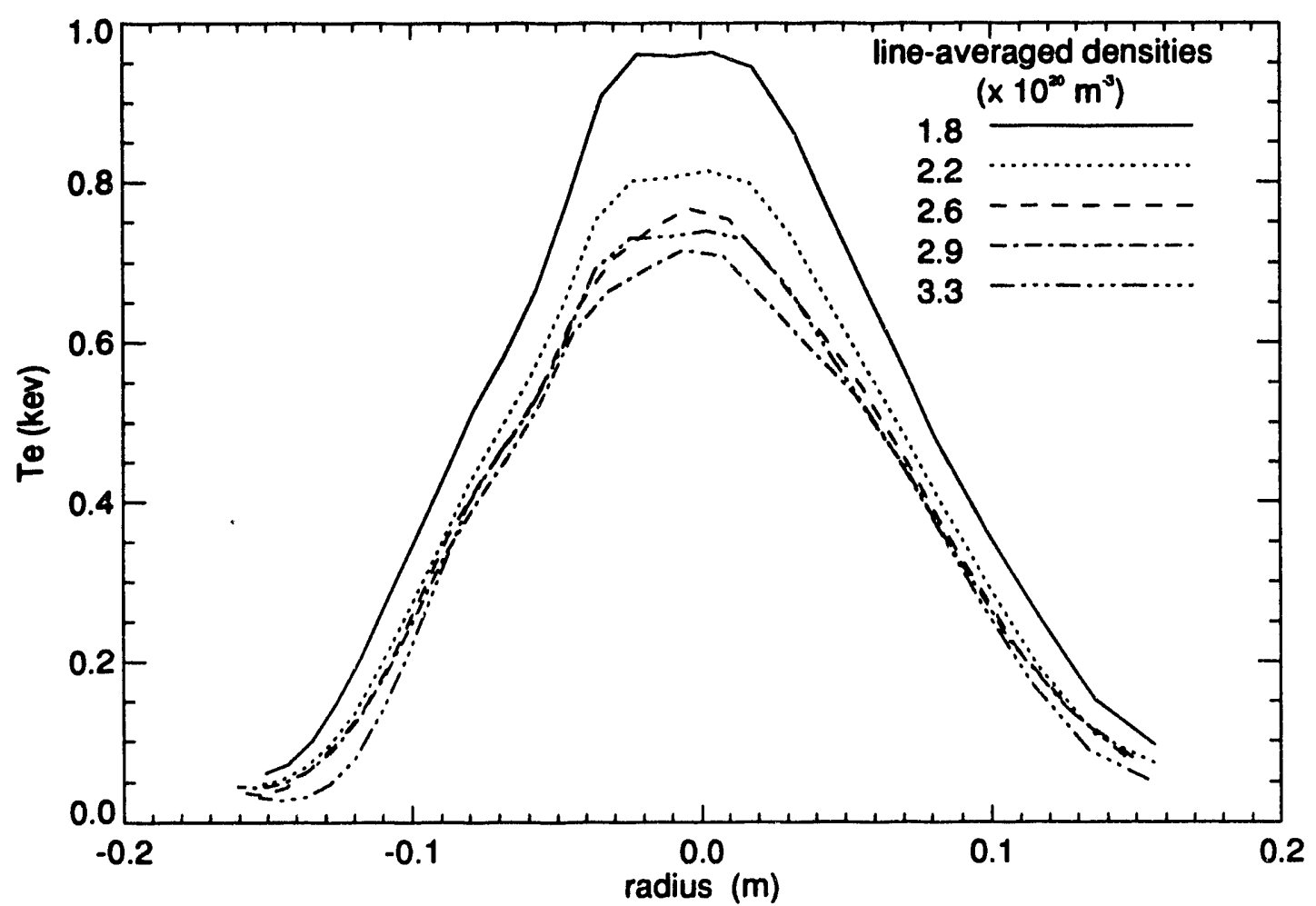

Figure 4.10: Black body temperature profiles for $285 \mathrm{kA}$ plasmas at $280 \mathrm{msec}$. Outside of $\mathrm{r} / \mathrm{a}=0.8$ the 'mission is not optically black.

In fig. $4.1 \mathrm{C}$ black body temperature profiles are derived from second harmonic $\mathrm{x}$-mode radiation emission from $285 \mathrm{kA}$ shots at $280 \mathrm{msec}$, the middle of the density modulation transient. As the plasma density and temperature drop rapidly outside of $\mathrm{r} / \mathrm{a}=0.8$ the plasma emission no longer behaves as optically thick and the temperature becomes unreliable. Inside this region the values of $\mathrm{T}_{e}$ should approach closely the actual plasma electron temperature. The five $\mathrm{T}_{e}$ profiles have similar widths as would be expected for shots having the same $\mathrm{q}_{a}$ on Alcator C. The profiles for $\bar{n}_{e, 20}=1.8$, 2.2, 2.6 and 2.9 are from shots belonging to the group used for particle transport analysis. Temperature profiles measured for other shots grouped with the same data point are essentially identical at this same time. The relative accuracy of the black body temperatures measured with the Michelson interferometer should be accurate to the level of a few percent from shot to shot [62]. The accuracy of the absolute magnitude of the measurement is considerably less. In the study of the variations of these profiles from shot to shot we are primarily concerned with the relative accuracy, 
which is quite good.

Because the Michelson interferometer was not available on the day the gas modulation data at $\bar{n}_{e, 20}=3.3$ and 3.6 was taken, an effort was made to reproduce the discharge conditions of the $\bar{n}_{e, 20}=3.3$ data. The temperature profile for the $\bar{n}_{e, 20}=$ 3.3 data is from shot 14016 and was taken on the same day as for the rest of the the profiles in fig. 4.10. The plasma current for this shot was just $6.6 \%$ higher than the average value of the $\bar{n}_{e, 20}=3.3$ data group at the start of the gas puff. The density at that time was within 1.5 standard deviations of the average value in that same group of shots. Also the time history of the evolution of $\bar{n}_{e}$ in this shot prior to the puff is very close to that of the $\bar{n}_{e, 20}=3.3$ group. On Alcator C plasma characteristics on similar shots are generally quite reproducible from day to day. The data used for the $\bar{n}_{e, 20}=3.3$ profile is from the same density scan on the same day as for the other profiles. The principal difference was the higher density. There is an obvious trend towards lower electron temperatures as the density is increased.

\subsubsection{Variation of Particle Transport Coefficients}

Perturbed particle transport coefficients were obtained for the $285 \mathrm{kA}$ data points using the multidimensional linear regression analysis routine outlined in sec. 3.4. Profiles of $\mathrm{D}(\rho)$ and $\mathrm{V}(\rho)$ are shown in fig. 4.11. A dramatic increase in the transport coefficients is seen in the outer portion of the profiles at $\mathrm{n}_{e, 20}=3.3$, where the density is at the limit value predicted by the Greenwald scaling. The increase in both $\mathrm{D}$ and $\mathrm{V}$ is most pronounced at the outer edge of the region analyzed, but is apparent over a region which extends well into the plasma core. Near the center of the plasma, the coefficient values are not increased over the values at lower densities.

The increase in the perturbed transport coefficients is consistent with density limit behavior. Although both $\mathrm{V}$ and $\mathrm{D}$ increase together, the increase in $\mathrm{D}$ appears to be somewhat more pronounced. As $\mathrm{D}$ increases relative to $\mathrm{V}$, the increase in outward flux generated by a small increase in the local density gradient becomes relatively larger than the increase in inward flux due to a small increase in density. The local density gradient cannot increase as fast as the local density so the density profile 

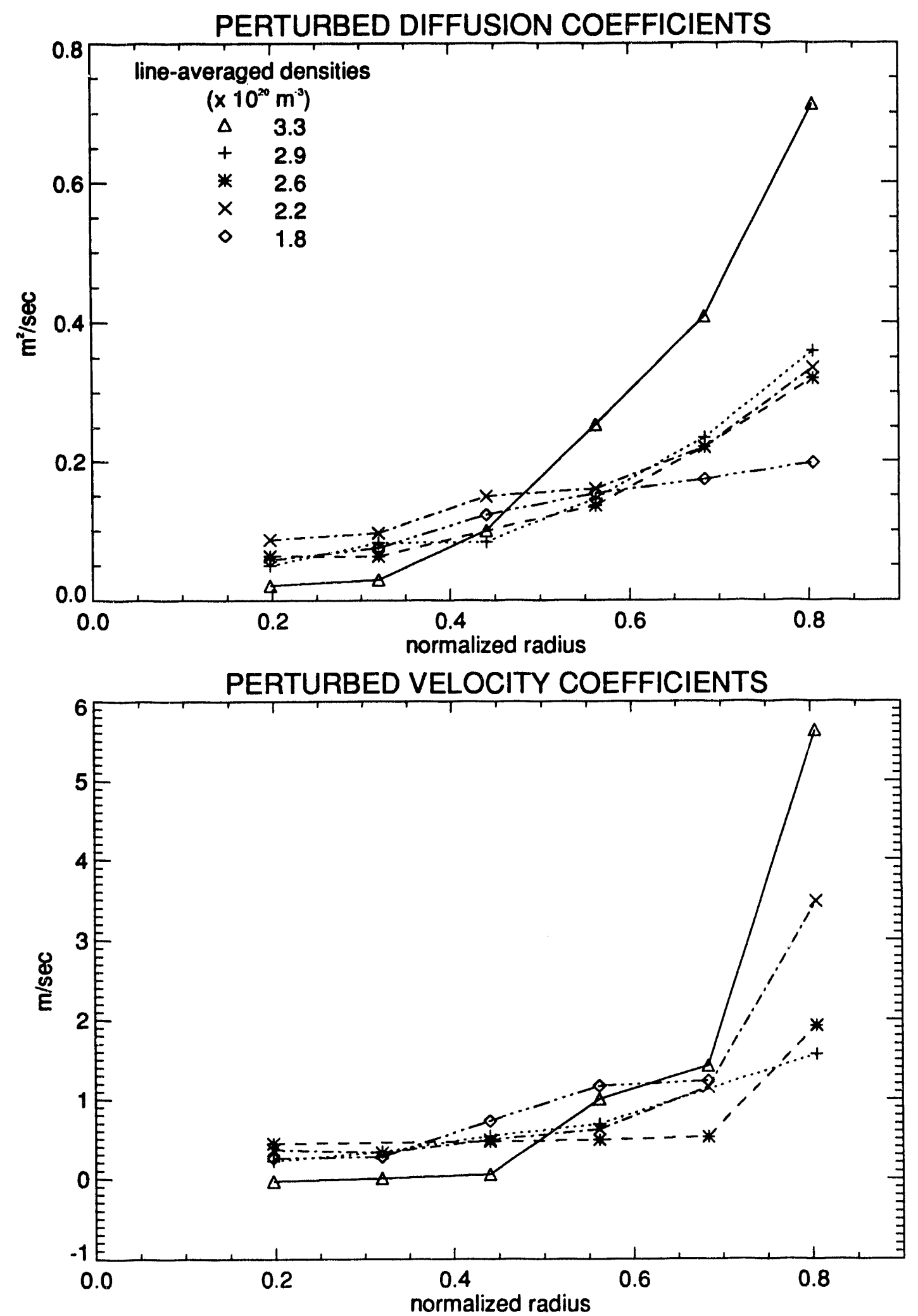

Figure 4.11: Particle transport coefficients overlaid from $285 \mathrm{kA}$ shots with $\mathrm{n}_{e, 20}=$ $1.8,2.2,2.6,2.9,3.3$. 
saturates.

The behavior of the perturbed transport coefficients near the density limit is qualitatively consistent with results of the pellet fuelling experiments at MIT. The increase in the transport coefficients occurs rapidly in the vicinity of the density limit. The region where a large increase occurs in the coefficients occurs extends well into the core of the plasma. Recall that in the pellet experiments the density decay time decreased dramatically near the density limit. This decrease decay time was symptomatic of a deterioration of particle confinement in the plasma core.

The profiles of $D(r)$ for the three other plasmas well within the saturated ohmic confinement regime $\left(\bar{n}_{e, 20}=2.2,2.6,2.9\right)$ are very similar. The plasma with $\bar{n}_{e, 20}=$ 1.8 is just below the range of densities associated with saturated ohmic confinement in Alcator C. Data from the shots at $\bar{n}_{e, 20}=3.6$ on MTX could not be used to determine transport coefficients because of the breakup of many of the FIR chords and the large amount of noise present.

A plot of global energy confinement times determined from the diamagnetic loop for the $285 \mathrm{kA}$ shots is given in fig. 4.12. Values of global energy confinement time are also estimated from ONETWO simulation which uses electron temperature profile, density profile and other data. Ion temperature profiles are calculated assuming ion neoclassical thermal conductivity multiplied by a constant determined in order to match the neutron production rate. The ion neoclassical multipliers were determined as functions of line averaged density and toroidal field strength at MIT. For these gas modulation and other shots listed in table 4.2 the global energy confinement times from the DML and ONETWO agree to within 10\%, and often agree to within a few percent.

The global energy confinement time is nearly independent of $\bar{n}_{e}$ in the saturated ohmic confinement regime. This same behavior is observed in the profile of $D(r)$ over most of this regime. A most significant observation is that there is no significant deterioration of the global energy confinement time near the density limit, even though the particle transport coefficients increase rapidly there. This may strike the reader as unusual since at densities well below the density limit $D$ and $1 / \tau_{E}$ have been reported 


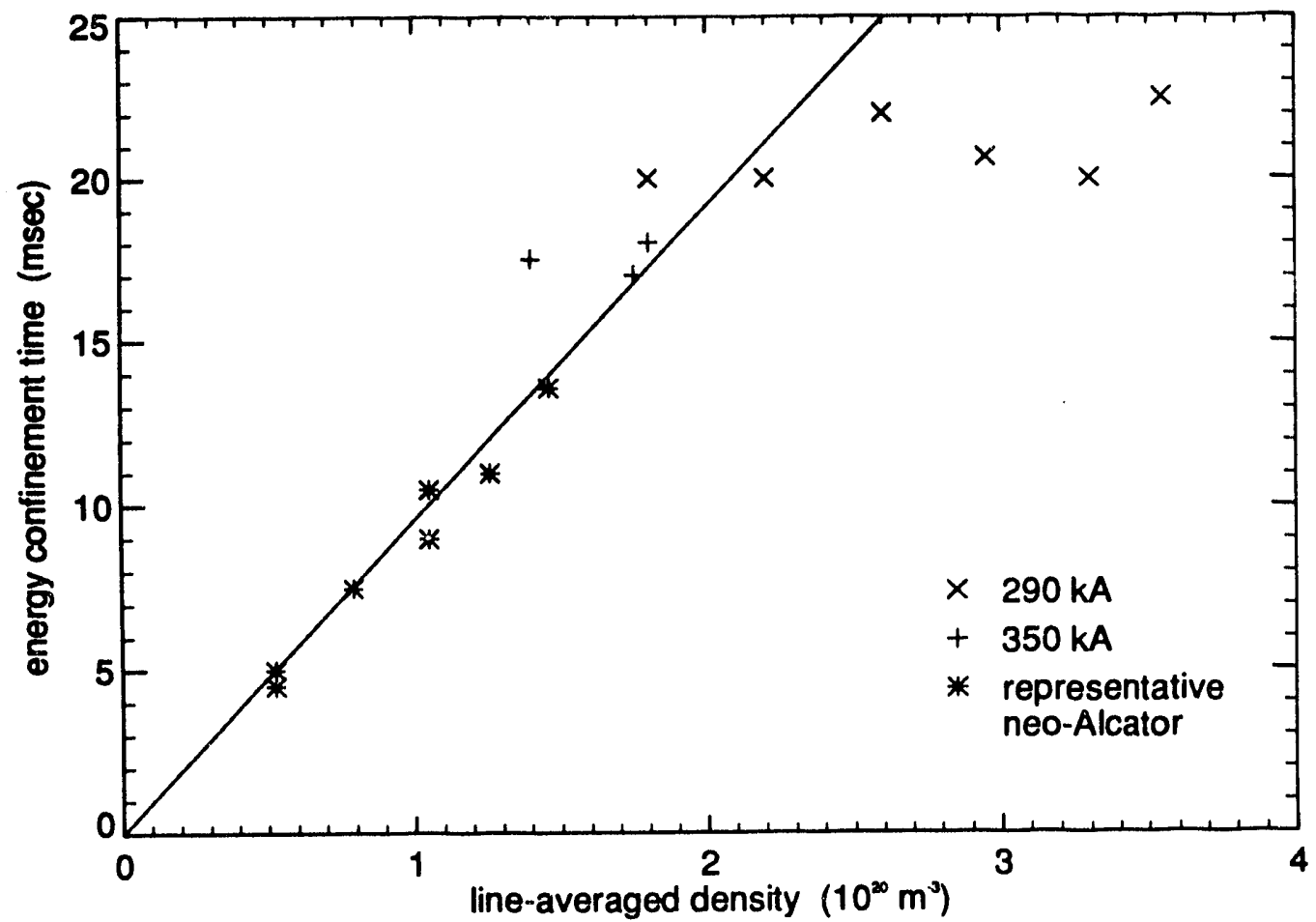

Figure 4.12: Global energy confinement times measured by the diamagnetic loop.

\begin{tabular}{|ccrr|}
\hline shot number & time & DML energy & ONETWO energy \\
\hline 13774 & $300 \mathrm{msec}$ & $9500 \mathrm{~J}$ & $8930 \mathrm{~J}$ \\
13256 & $310 \mathrm{msec}$ & $11550 \mathrm{~J}$ & $11200 \mathrm{~J}$ \\
13495 & $260 \mathrm{msec}$ & $7500 \mathrm{~J}$ & $7900 \mathrm{~J}$ \\
13522 & $280 \mathrm{msec}$ & $11450 \mathrm{~J}$ & $11100 \mathrm{~J}$ \\
13524 & $240 \mathrm{msec}$ & $5800 \mathrm{~J}$ & $5450 \mathrm{~J}$ \\
14036 & $315 \mathrm{msec}$ & $13200 \mathrm{~J}$ & $12200 \mathrm{~J}$ \\
13938 & $240 \mathrm{msec}$ & $14000 \mathrm{~J}$ & $13950 \mathrm{~J}$ \\
13257 & $280 \mathrm{msec}$ & $11450 \mathrm{~J}$ & $11050 \mathrm{~J}$ \\
14095 & $240 \mathrm{msec}$ & $10650 \mathrm{~J}$ & $9980 \mathrm{~J}$ \\
13550 & $320 \mathrm{msec}$ & $8700 \mathrm{~J}$ & $8300 \mathrm{~J}$ \\
\hline
\end{tabular}

Table 4.2: Global Energy Confinement Times determined From DML and ONETWO 
to behave in a similar manner.

Gentle [80] used gas modulation data from the four interferometer chords on ASDEX to determine averaged values of $\mathrm{D}$ and $\mathrm{V}$ for the center and outer portions of the discharges. The values of $\mathrm{D}$ in the outer portion of the discharges was found to correlate with the inverse of global energy confinement time. In the ohmic linear confinement regime $\mathrm{D}_{e}$ decreased in the outer plasma while $\tau_{e}$ increased with increasing density. At higher densities, in the saturated ohmic confinement regime, both global energy confinement and $\mathrm{D}$ in the outer plasma showed very little variation with line-averaged density. A similar trend was observed in TEXT.

In an earlier study [23] gas modulation data from the 6 interferometer chords available on TEXT at the time was fit assuming profiles of transport coefficients parameterized each by a single value. The diffusion coefficient $\mathrm{D}$ was taken as spatially constant and $\mathrm{V}$ was given by $\mathrm{V}(\mathrm{r})=\mathrm{V}_{0} \cdot \mathrm{r} / \mathrm{a}$, with $\mathrm{V}_{o}$ the single adjustable constant. Because of the limited number of FIR chords available then it was not possible to obtain detailed radial profiles of the transport coefficients. The model with these single parameter transport coefficients was able to fit most TEXT data well. The exception was shots near the density limit. Data from these shots was often not well fit by single values for $\mathrm{D}$ and $\mathrm{V}$. Although an analysis with a more general form for the transport coefficient was not undertaken, comparison of the experimental data with simulation for various values of $\mathrm{D}$ and $\mathrm{V}$ showed that the central core of the plasma was characterized by a smaller value of $\mathrm{D}$ with $\mathrm{V} \sim 0$ (or neoclassical) [23]. The outer region was characterized by higher values of $\mathrm{D}$ and $\mathrm{V}$ which maintained the ratio required for the equilibrium profile. These were larger than values in shots at similar densities with different currents which were not near the density limit.

\subsection{Density Limit at Higher q}

At a higher value of $\mathrm{q}_{a}(=5.7), \mathrm{I}_{p}=220 \mathrm{kA}$ a much larger $\mathrm{D}_{\alpha}$ signal was obtained at densities approaching the limit values. The $\mathrm{D}_{\alpha}$ detector, which views along the tokamak equator, was often saturated during shots at $\bar{n}_{e, 20}=1.8,2.0$ at this current. 
As occurred at the higher current $(285 \mathrm{kA})$ the plasma density responded less to a gas puff as the density limit was approached. The saturated $D_{\alpha}$ signal implied greatly increased recycling and an associated decrease in the global particle confinement time. Under these conditions, dominated by recycling, it became difficult to increase the plasma density beyond $\bar{n}_{e}=2.0 \times 10^{20} \mathrm{~m}^{-3}$. The gas puff amplitude was increased further but with little apparent increase in the size of the density modulation. Eventually FIR chord signals became increasingly noisy and began to break up. A density limit was reached at $\bar{n}_{e, 20}=2.05$ for $\mathrm{I}_{p}=220 \mathrm{kA}$, less than the value given by the Greenwald scaling.

The $60 \mathrm{~Hz}$ noise became quite prominent in these shots at $\bar{n}_{e, 20}=1.8,2.0$, having an amplitude comparable than that from the density modulation of the bulk plasma due to the gas puff. For these shots, even with filtering, it became impossible to render insignificant the contamination of the data. Results obtained from the analysis of these shots depended sensitively upon the length of the time segment analyzed, due to the prominence of the $60 \mathrm{~Hz}$ component. Thus data from shots at $\bar{n}_{e, 20}=1.8$, $2.0, \mathrm{I}_{p}=220 \mathrm{kA}$ could not be used to obtain transport coefficients.

\subsection{Relations Between Transport Coefficients}

The general rise in coefficient values toward the outside of the plasma is a universal feature of all gas modulation results from MTX. Fig. 4.13 shows the ratio of the values of $(\mathrm{aV} / \mathrm{D})$ versus the inverse of the density gradient scale length normalized to minor radius for $0.4 \leq \mathrm{r} / \mathrm{a} \leq 0.8$ for gas modulation data at $285 \mathrm{kA}$. Values fall in the range $1 / 4$ to 1 times the value of $a / L_{n}$ expected for the balance of equilibrium transport coefficients obtained from eq. 2.2 in the absence of a source. This inequality implies that the terms stemming from the dependences of the transport coefficients on local parameters, including off diagonal terms, given in equations 2.14 and 2.15 play a significant role in determining the values of perturbed coefficients.

The perturbed electron diffusion coefficients obtained from density modulation experiments are frequently compared with values of electron thermal diffusivity ob- 


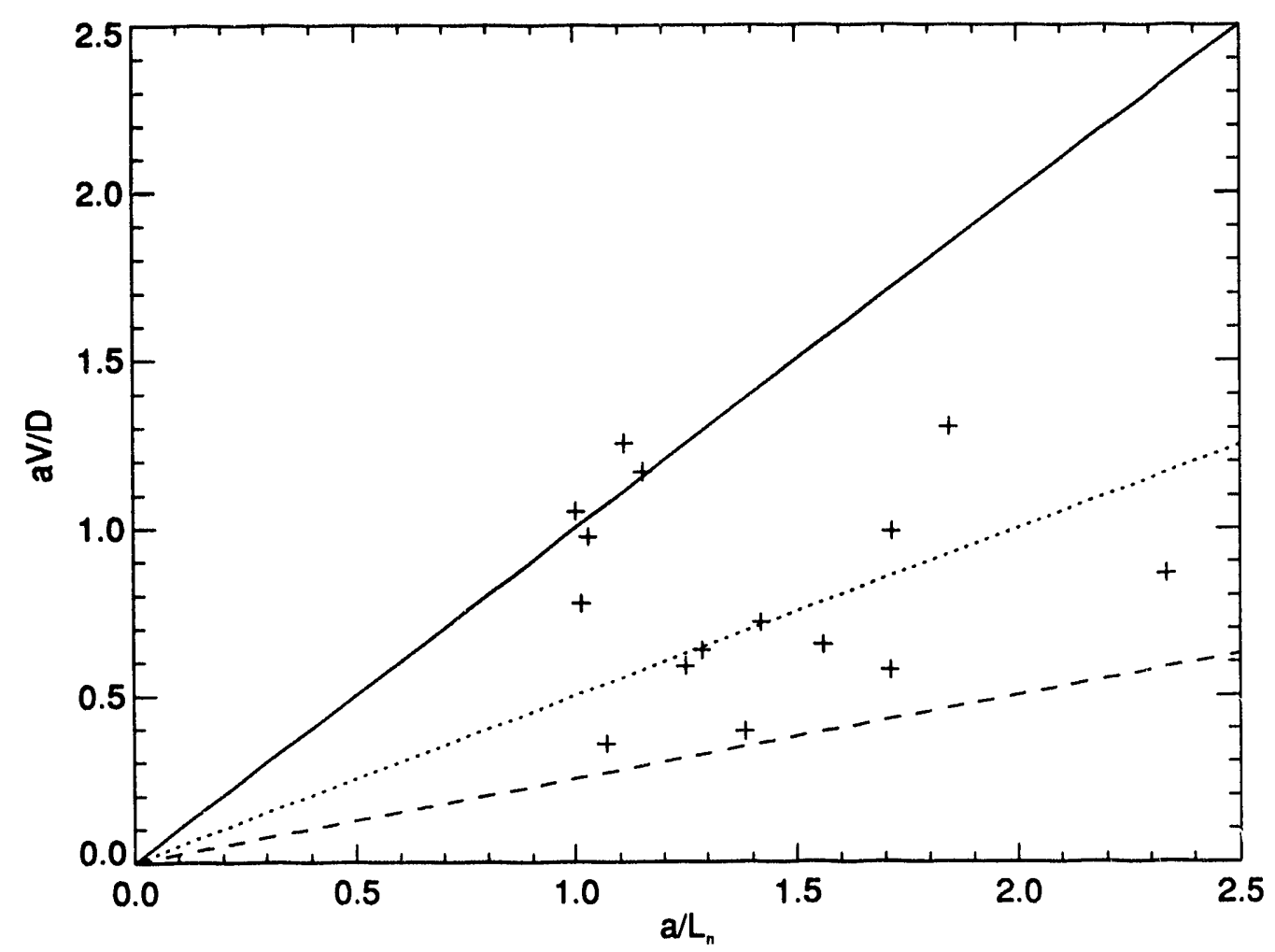

Figure 4.13: Values of $\mathrm{aV} / \mathrm{D}$ versus $\mathrm{a} / \mathrm{L}_{n}$ for $0.4<\mathrm{r} / \mathrm{a}<0.8$.

tained from heat pulse propagation measurements. On TEXT values of $\chi_{e} \sim 2-4$ times the values of $\mathrm{D}_{e}$ obtained with gas modulation experiments have been reported [23]. Values of $\chi / \mathrm{D} \sim 1$ were reported on TFTR [24], where these values of $\mathrm{D}$ were also obtained from gas modulation experiments. Values of D were obtained from modelling of the density decay following pellet injection on JET. These compared with the electron thermal diffusivity as $\chi_{e} / \mathrm{D} \sim 10$ [76].

The shape of the profile of D for MTX plasma with $\bar{n}_{e}=0.9, \mathrm{I}_{p}=220 \mathrm{kA}$ in fig. 4.19 bears resemblance to nrofiles of $\chi_{e}$ determined previously from analysis of sawtooth heat pulse propagation of Alcator $\mathrm{C}$ by Gomez [77]. The value of $\mathrm{D}$ at $\mathrm{r} / \mathrm{a}$ $=0.7$ is $\sim 3$ times as great as the value at $\mathrm{r} / \mathrm{a}=0.3$. Profiles of $\chi_{e}$ most often given by Gomez are the best fit of a parabolic functional form of $\chi_{e}(r)$ to the data. The ratio of $\chi_{e}(r / a=0.7)$ to $\chi_{e}(r / a=0.3)$ for the parabolic fits are typically of order 3 as well. Values of $\chi_{e}$ at three locations $(r / a=0.3,0.5,0.7)$ are reported by Gomez as a function of line-averaged density. For most of the densities in the $285 \mathrm{kA}$ scan the ratios of the local values $\chi_{e} / \mathrm{D}$ fall in the range 2-5. The exception occurs near the 
density limit, where $\mathrm{D}$ is increased.

At low densities Alcator $\mathrm{C}$ plasmas generally exhibit Neo-Alcator scaling of the global energy confinement time, $\tau_{E}=0.192 \bar{n}_{14} R^{2.04} a^{1.04}$. As the line density is raised to $\bar{n}_{e} \simeq 2.0 \times 10^{20} \mathrm{~m}^{-3}$ the energy confinement in Alcator $\mathrm{C}$ saturates in gas-fuelled plasmas. In the saturated ohmic confinement regime global energy confinement time shows little dependence on line-averaged density with gas fuelling. Gomez reports no saturation in $\chi_{e}$ in this regime. Rather it continues to decrease with increasing density, scaling roughly as $\sim 1 / \bar{n}_{e}$. The saturation of global energy confinement time is due to anomalous thermal conduction losses in the ion channel [78]. When pellets are used to produce peaked density profiles, improvement in the global energy confinement time results as the ion thermal conductivity decreases to approximately the neoclassical value [78].

\subsection{Anomalous Inward Convection}

The profile of the inward convection velocity is shown for $\bar{n}_{e}=2.2 \times 10^{20} m^{-3}, \mathrm{I}_{p}=285$ $\mathrm{kA}$ in fig. 4.14 and compared with the calculated values of the neoclassical Ware pinch [79]. The perturbed inward convection is anomalously large, particularly in the outer region of the plasma. Functional dependences of D on plasma parameters cause it to contribute to the perturbed convection coefficient through terms such as $\frac{\partial D}{\partial n}<\nabla n>$. It has been argued that this is the cause of the anomalously large inward pinch obtained in perturbation experiments [24]. The steady state particle flux was calculated with ONETWO for the above plasma using a conservatively large estimate of the global particle confinement time of $3 \mathrm{msec}$. During the modulation the sum of the perturbed and calculated steady state fluxes is inward for a time for $\mathrm{r} / \mathrm{a} \leq 0.8$. Since the density gradient is radially inward, the diffusive component of the flux is outward at any instant. Thus a net inward particle flux observed in the ohmic MTX plasma must be due to an anomalous inward convection process. We see that fractionally small changes in $\tilde{n}$ and $\nabla \tilde{n}$ are capable of causing the particle flux to change sign in the interior of MTX plasmas. Anomalous inward convection was 


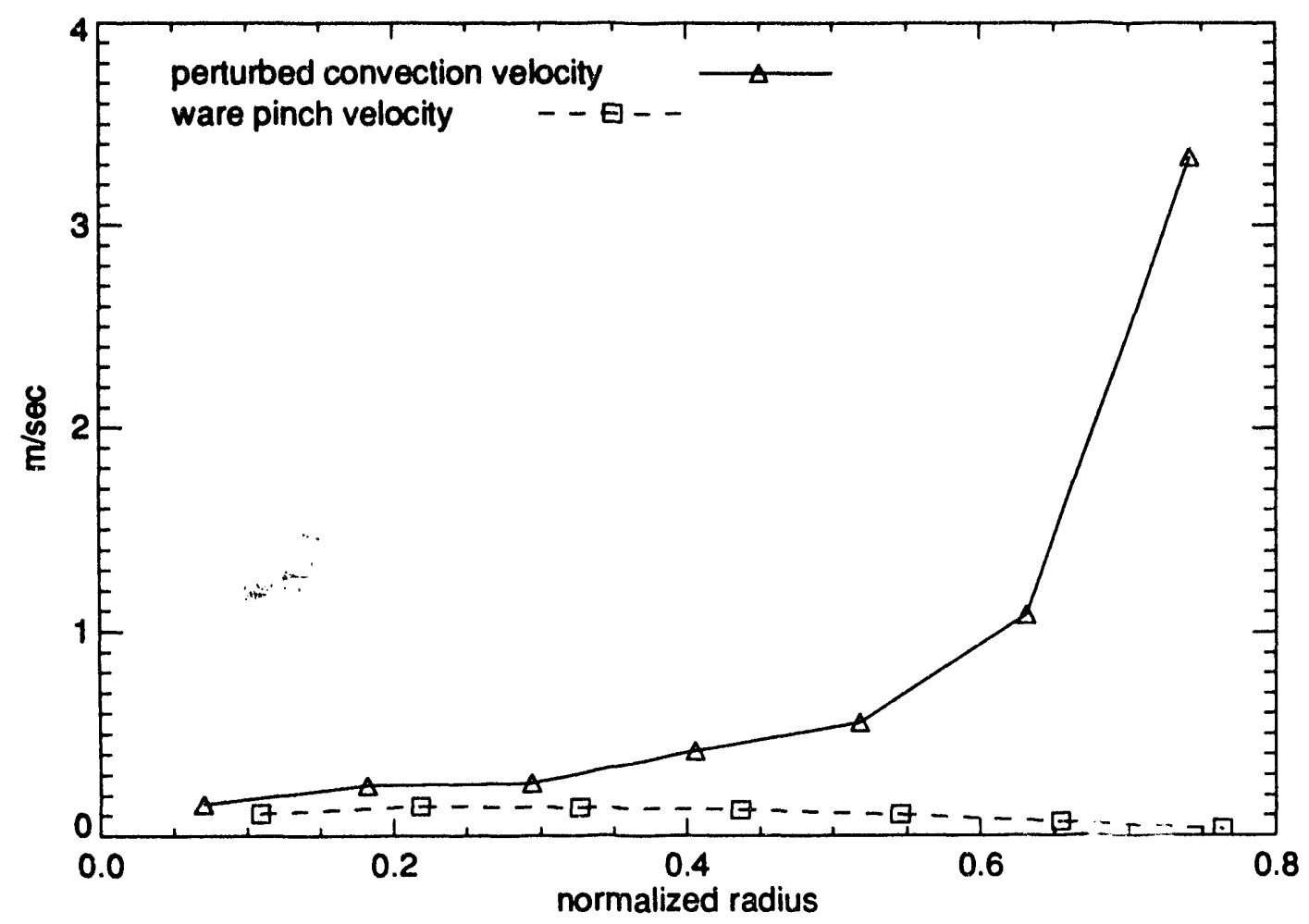

Figure 4.14: Measured perturbed convection velocity versus Ware pinch.

found on ASDEX as well [80]. In the outer half of the ASDEX plasma it is always large, much larger than neoclassical, but it is more variable in the interior.

\subsection{Results with Periodic Modulation}

Shot 13301 is near the highest density at which periodic modulation is useful on MTX. The background density fit in fig 4.15 shows a central density of $2.0 \times 10^{20} \mathrm{~m}^{-3}$ corresponding to a line averaged density of $\bar{n}_{e, 20}=1.4$. The amplitudes and phases of the $15 \mathrm{~Hz}$ fundamental component of the chord signals relative to that for the center chord are shown as diamonds in fig 4.16. Much of the apparent asymmetr.' in these profiles is due to the plasma offset and the Shafronov shift. The analysis routine described in the previous chapter is used to determine $\tilde{n}_{e}(\rho, \omega)$ from which the time evolution of the $15 \mathrm{~Hz}$ component of the modulation is obtained simply as $\left.\tilde{n}_{e}(\rho, t)\right|_{15 H z}=\tilde{n}_{e}(\rho, \omega) e^{i \omega t}$. Even this fundamental component, shown at several time steps in fig. 4.17 , is largely damped as it propagates inward. This damping was also 

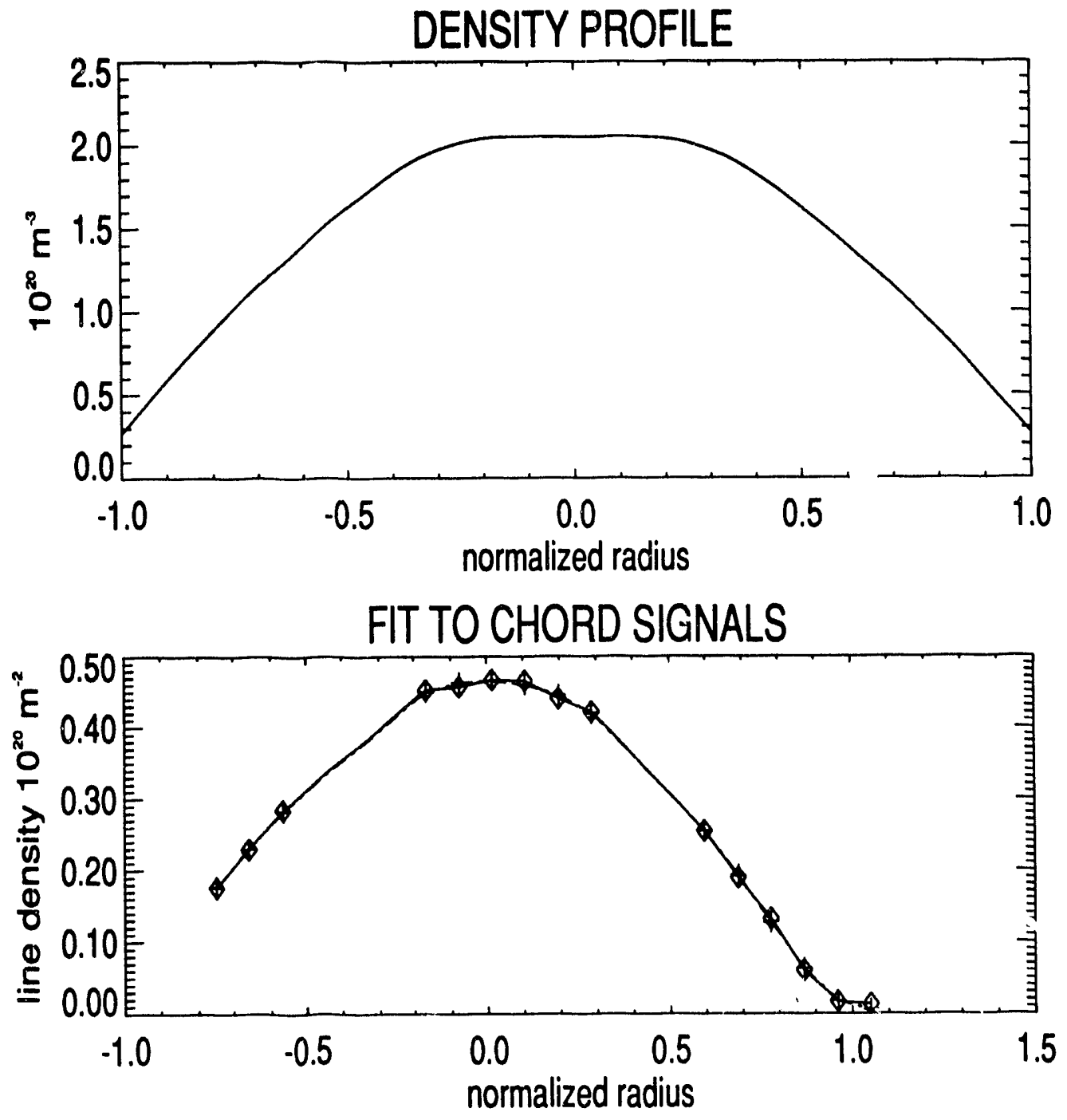

Figure 4.15: Background density fit for shot 13301 at $150 \mathrm{msec}$. 


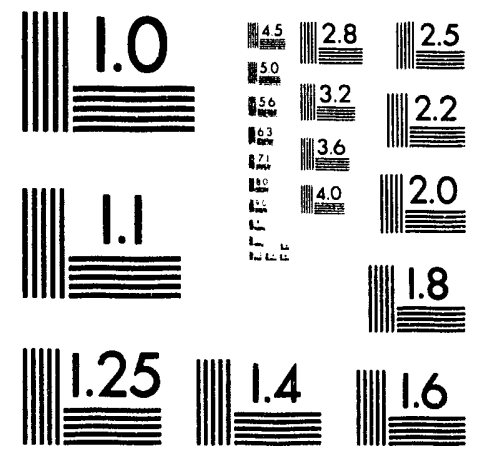



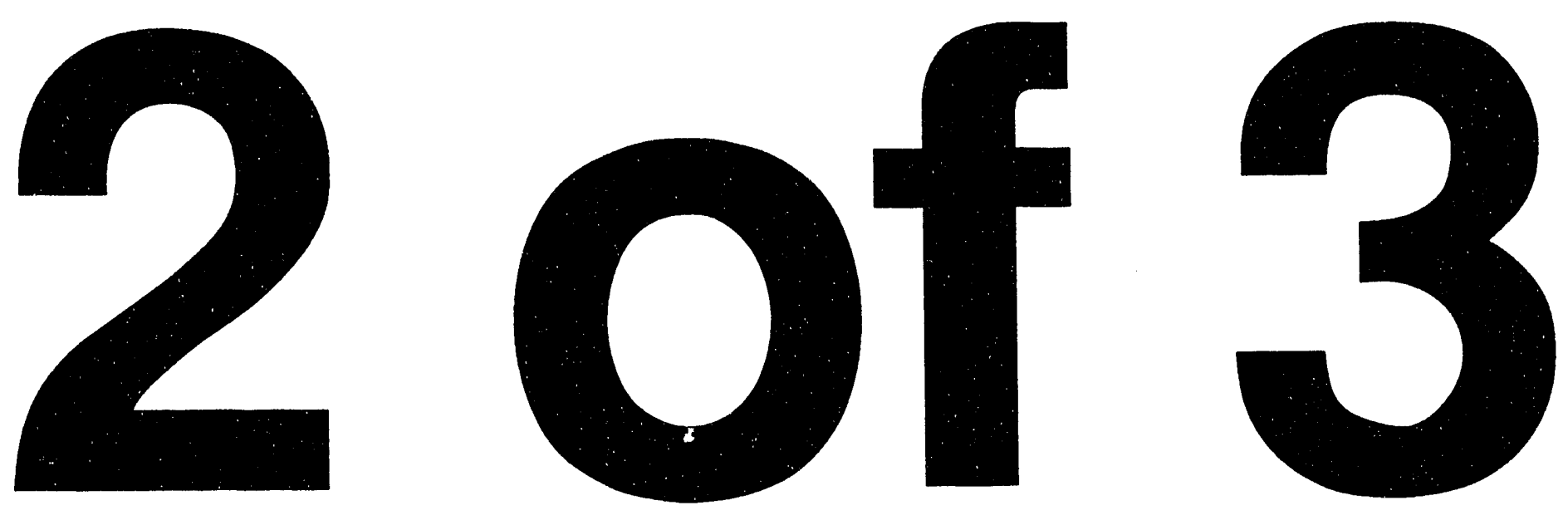

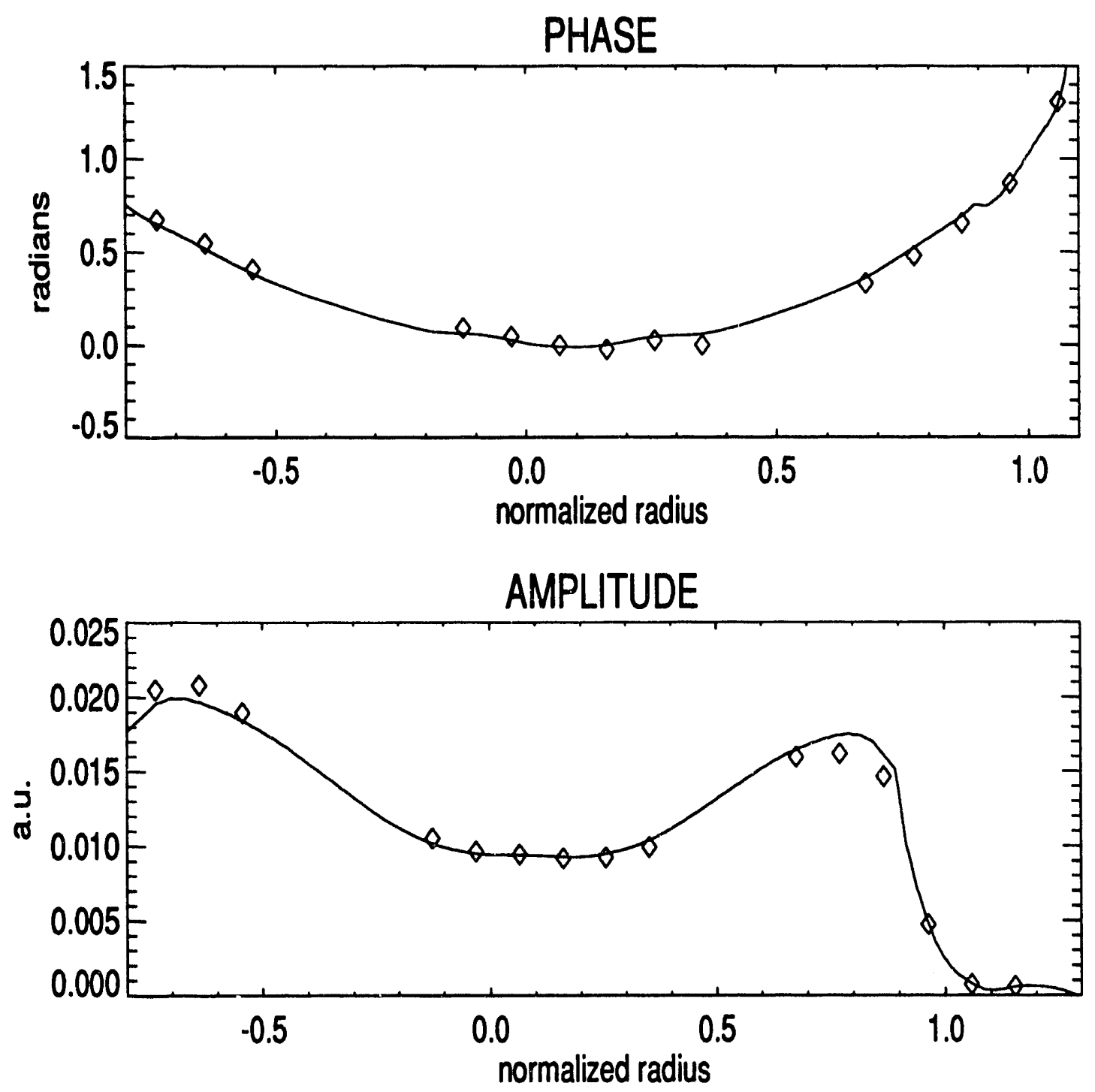

Figure 4.16: Amplitude and phases of $15 \mathrm{~Hz}$ components of the chords relative to the central chord for shot 13301 . 


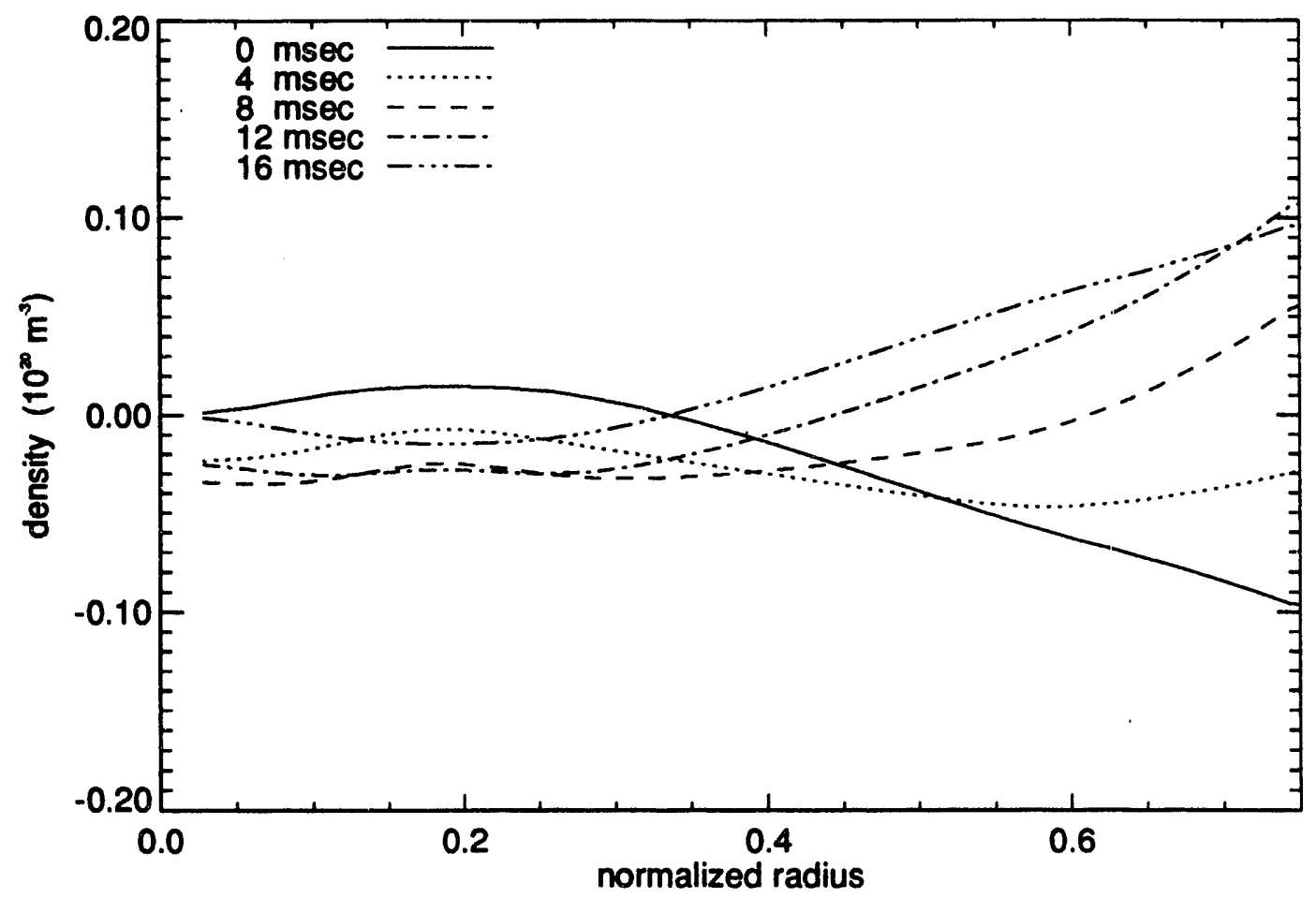

Figure 4.17: Fundamental $15 \mathrm{~Hz}$ component of modulation in shot 13301 at several time steps. 
apparent from the relative modulation amplitudes of the chords in fig 4.16 as well. This damping results because the modulation frequency is at the high end of the useful frequency range. Because of the length of shots in MTX, $15 \mathrm{~Hz}$ is the lowest modulation frequency which yields the desired minimum of 3 periods during the current flat top. The amplitude and phases of the equivalent chord signals resulting from the fit are superimposed in fig 4.16. The portion of the asymmetry in the data that is not explained by the fit is likely due to the ever present density asymmetries near the limiter and in the scrape-off layer. Since the density wave rapidly decays as it propagates inward from the edge in this case, edge effects are more pronounced. Yet, even with with the strong damping present in this case the influence of the edge asymmetry is modest.

For the series of shots at lower density, $\bar{n}_{e}=0.9 \times 10^{20} \mathrm{~m}^{-3}, \mathrm{I}_{p}=210 \mathrm{kA}$ (shots 13522-13527) the same $15 \mathrm{~Hz}$ fundamental frequency was used. Relative amplitudes and phases of the chord modulations are shown as diamonds in fig 4.18. The profile of the relative chord component amplitudes is less hollow than in the previous example. This is an indication that particle transport coefficients are larger in this plasma so that the modulation frequency is closer to the optimum value. The fundamental component of the density wave is only weakly damped as its propagates inward.

Equivalent chord amplitudes and phases for the fit of the analysis routine to the fundamental component are superimposed in fig. 4.18. The portion of the asymmetry not explained by the fit is rather small, within the statistical uncertainties associated with the coefficients for the chord signals. Because the asymmetry of the signal is small and well fit by the model using symmetric density on flux surfaces, we can infer that boundary plasma asymmetries have a minor influence on the chord signals here. Contributions to the modulated chord signal from variations in the bulk plasma regions, where the density perturbations are largest, predominate over contributions from the lower density edge regions. Thus in gas modulation studies of particle transport it is advantageous to perturb the density profile by a similar fractional amount across the column by choosing the modulation time scale properly in order to minimize the impact of poloidal asymmetries in the boundary plasma on the 

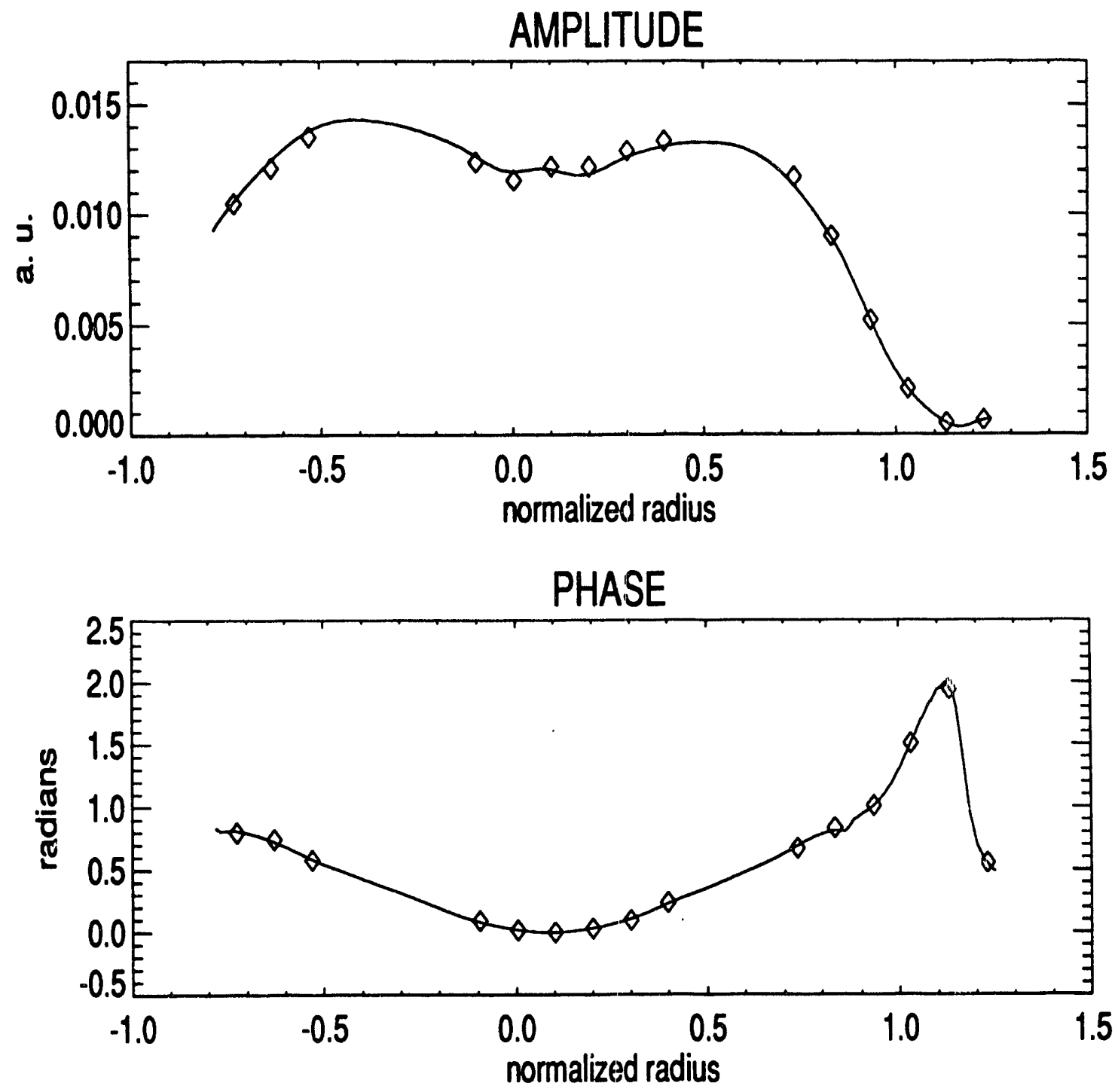

Figure 4.18: Relative amplitudes and phases of $15 \mathrm{~Hz}$ fundamental modulation component of shot 13527 

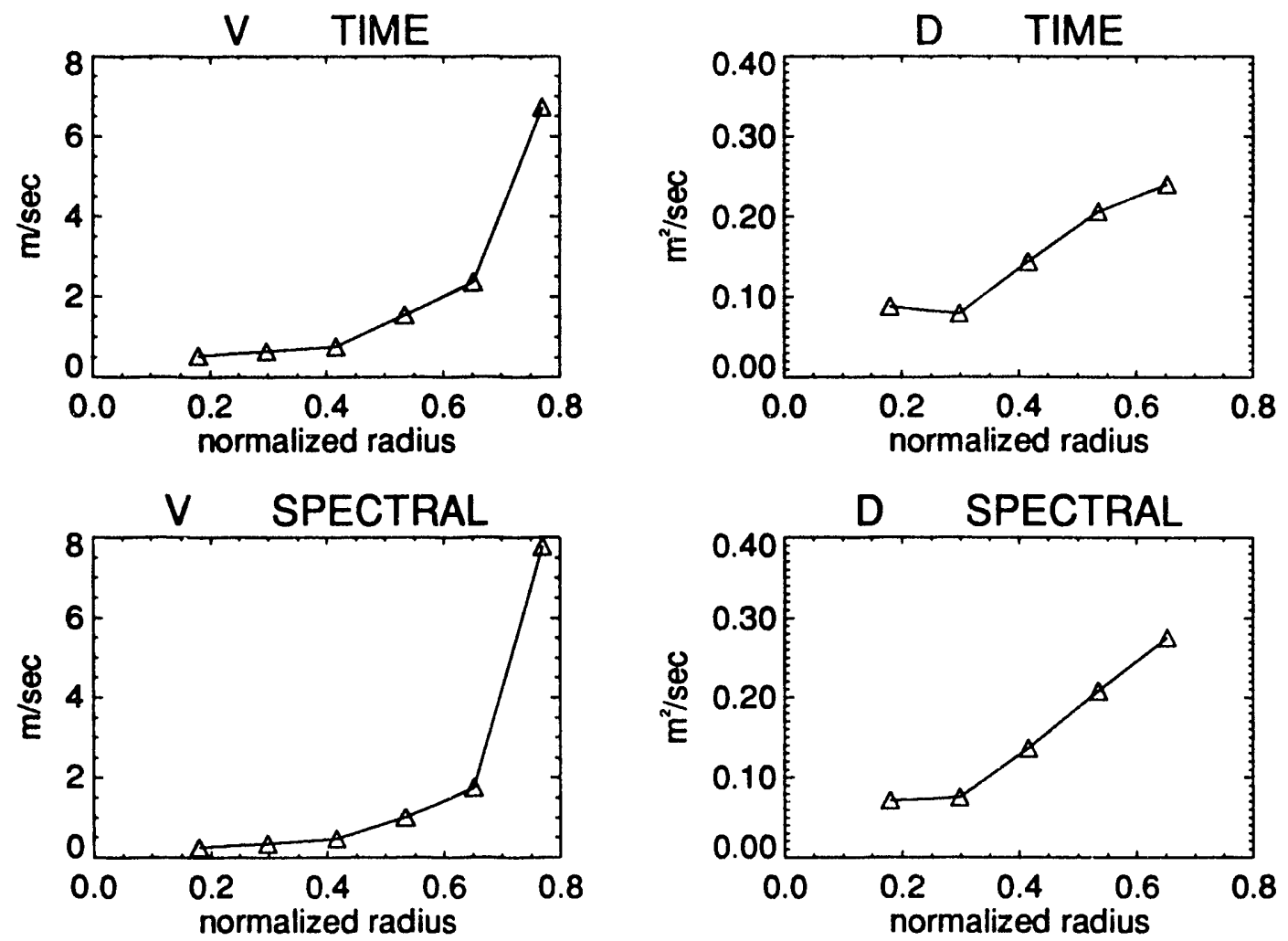

Figure 4.19: Comparison of transport coefficients obtained from spectral and time domain analysis of shots $13522-13527$.

measurement.

\subsection{Comparison of Results form Spectral/Time Domain Analysis}

Transport coefficients obtained from the spectral analysis method applied to the discharges in the last section are presented fig. 4.19. This same data was analyzed in the time domain by summing the signals over each of the three periods of the gas puffs for all six shots, then applying the multidimensional linear regression analysis over the length of the modulation period. Values obtained for $\mathrm{D}(\mathrm{r})$, shown in fig. 4.19 are very close to the corresponding ones derived from spectral analysis. There is a larger difference between the corresponding profiles of $\mathrm{V}(\mathrm{r})$ in fig. 4.19. The spectral analysis uses only the fundamental harmonic component of the signal, while all low frequency information is used in the time domain analysis. The differences in the 
values of perturbed convection velocities resulting from the iwo methods could be due to differences in the relative magnitudes of perturbations to separate variables, such as $\tilde{T}$ and $\tilde{n}$, occurring in the higher harmonic components of the signal. As we saw in eq. 2.15 the effects of these off diagonal terms are manifest in the perturbed convection coefficients.

\subsection{Significance of Model}

In order to assess the significance of the particle transport coefficients obtained from the analysis we must consider whether the model for the flux fits the data credibly and also estimate the impact of experimental uncertainties on the transport coefficients. Detailed analysis of these points is given in Appendix $G$ with the principal results summarized here. Statistical analysis of data from the $285 \mathrm{kA}$ density scan indicates that the model $\tilde{\Gamma}=-D \nabla \tilde{n}-V \tilde{n}+\beta$ is a meaningful description of the modulation transient to a very high degree of confidence. In addition both the convective and diffusive terms are necessary components of the model since both $\tilde{n}$ and $\nabla \tilde{n}$ are significantly related to the flux and the quality of the fit is significantly reduced if either of the terms is omitted. Having established that the model for the flux is a statistically valid description which fits the data reasonably well, we can now consider the question of the accuracy of the results obtained.

\subsubsection{Error Propagation}

The plasma density is measured at only a fixed number of chord positions. For this reason an unique determination of the transport coefficients is not possible. A variety of systematic errors arise from effects such as uncertainty in plasma position and geometry. When longer time spans are analyzed, as is the case with the spectral analysis method, errors due to slow changes in the plasma background conditions can become more important. With linear regression analysis it is possible to calculate uncertainties in the value of transport coefficients due to random variations in the data. This statistical error is however only a portion of the aggregate error which, as 


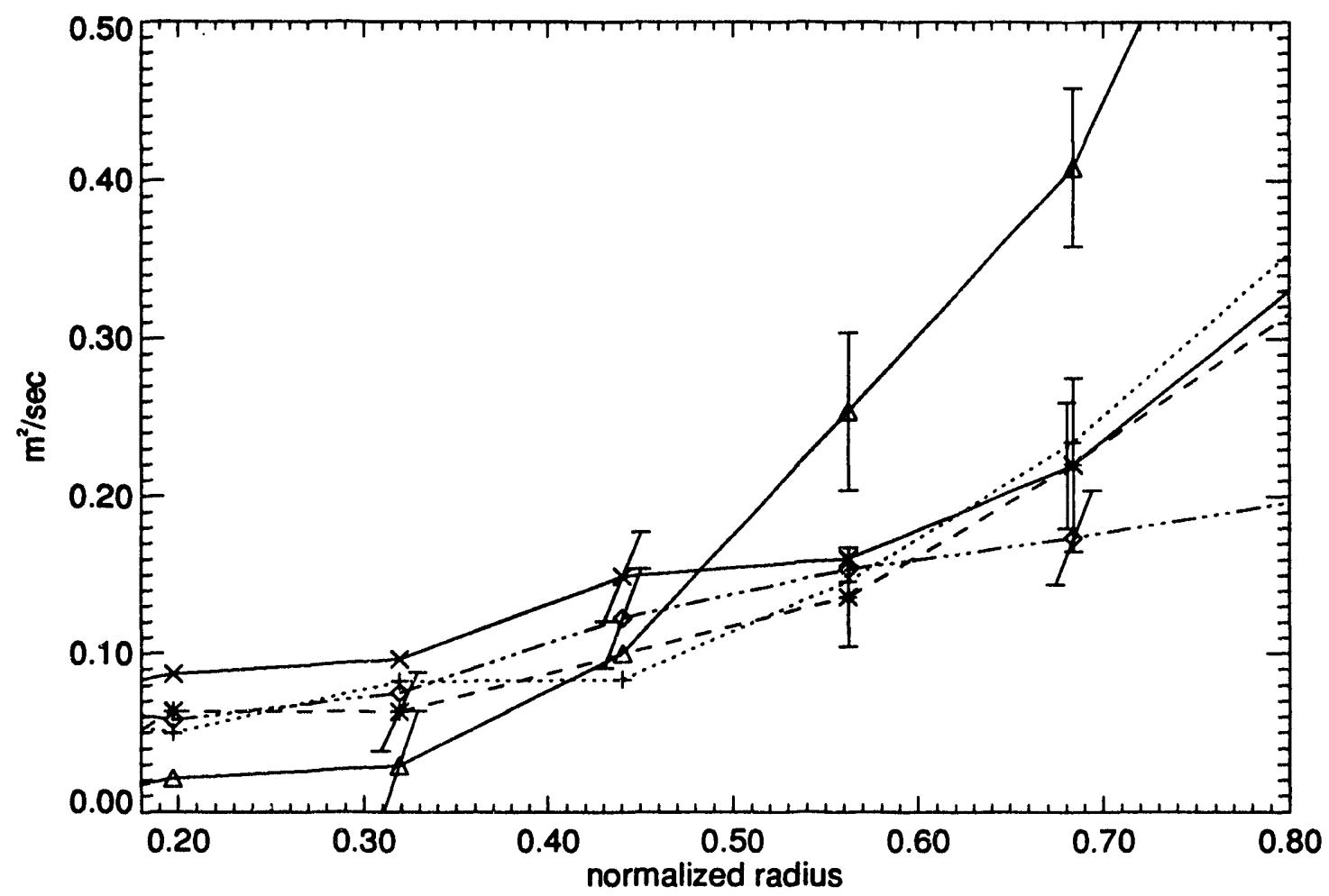

Figure 4.20: Representative uncertainties in diffusion coefficient values for plasma with $\mathrm{I}_{p}=285 \mathrm{kA}$.

shown in Appendix G, is dominated by the systematic errors. Uncertainties in plasma position and geometry are the dominant sources of uncertainties in the transport coefficient values. These were estimated by varying the values of the three principal - fit parameters used in the data analysis routine (the plasma offset $\delta_{0}$, the Shafronov shift $\epsilon_{0}$ and the flux surface geometry parameter $\alpha$ in eq. 3.3) over a range of uncertainties associated with each. The overall uncertainties of $D(r)$ and $V(r)$ result from the combination of systematic and random errors. Examples of these are shown in fig. 4.20. In the outer region of the plasma uncertainties in D of order $15 \%$ are not unusual. Near the plasma center, where the values of D are rather smaller, the fractional uncertainties can be quite substantial. When the values of coefficients cover a sizable range across the plasma cross section the smaller values cannot be accurately determined. Their value are simply localized to a small range. The fractional uncertainties in $V(r)$ are generally larger than those for $D(r)$. This result was obtained even when analyzing simulated data having coefficient values like the ones determined for 


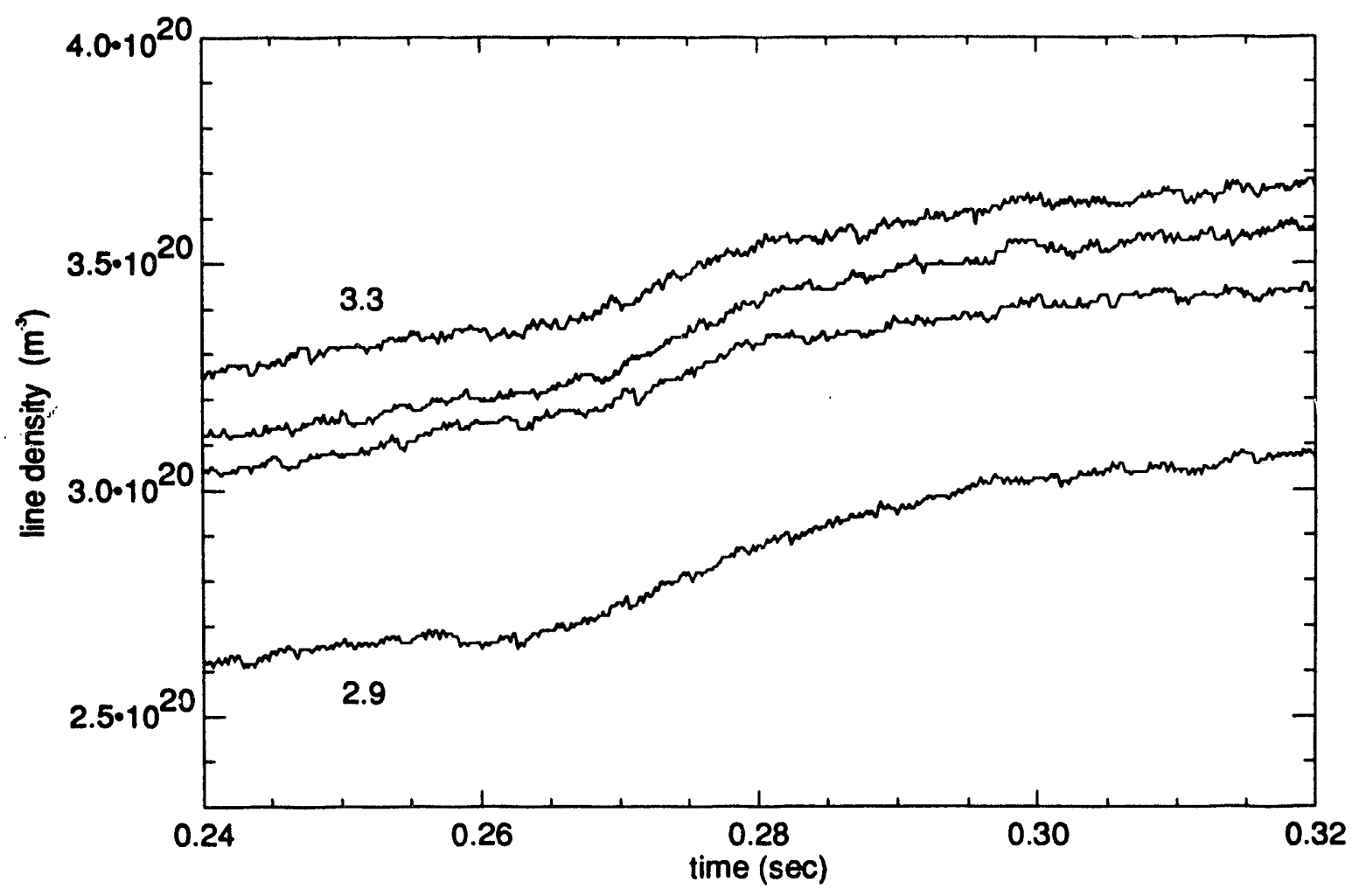

Figure 4.21: Central FIR chord signals during gas modulation at $\bar{n}_{e, 20}=3.3,2.9$.

\section{MTX plasmas.}

The changes in the transport coefficients in the outer plasma near the density limit are clearly significant. The substantial difference between the plasma transport behavior in shots near the density limit and at lower densities is evident in the unprocessed central chord FIR signals as well, shown in fig. 4.21. Signals from the gas valve pressure transducers for the shots at $\bar{n}_{e, 20}=z .9$ and 3.3 are shown in figure 4.22. The flow rate through the gas valve is essentially proportional to this pressure. The puffs are square waves starting at $260 \mathrm{msec}$. Both cases have the same duration and very similar amplitudes. The density rise during the puff in fig. 4.21 is more abrupt at $\bar{n}_{e, 20}=3.3$. Once the valve shuts at $280 \mathrm{msec}$, an inflection is pronounced in the curves for $\bar{n}_{e, 20}=3.3$. The $\bar{n}_{e, 20}=2.9$ data shows a more gradual change in slope following the puff. These differences are consistent with the enhanced transport rates in the outer plasma at the density limit. 

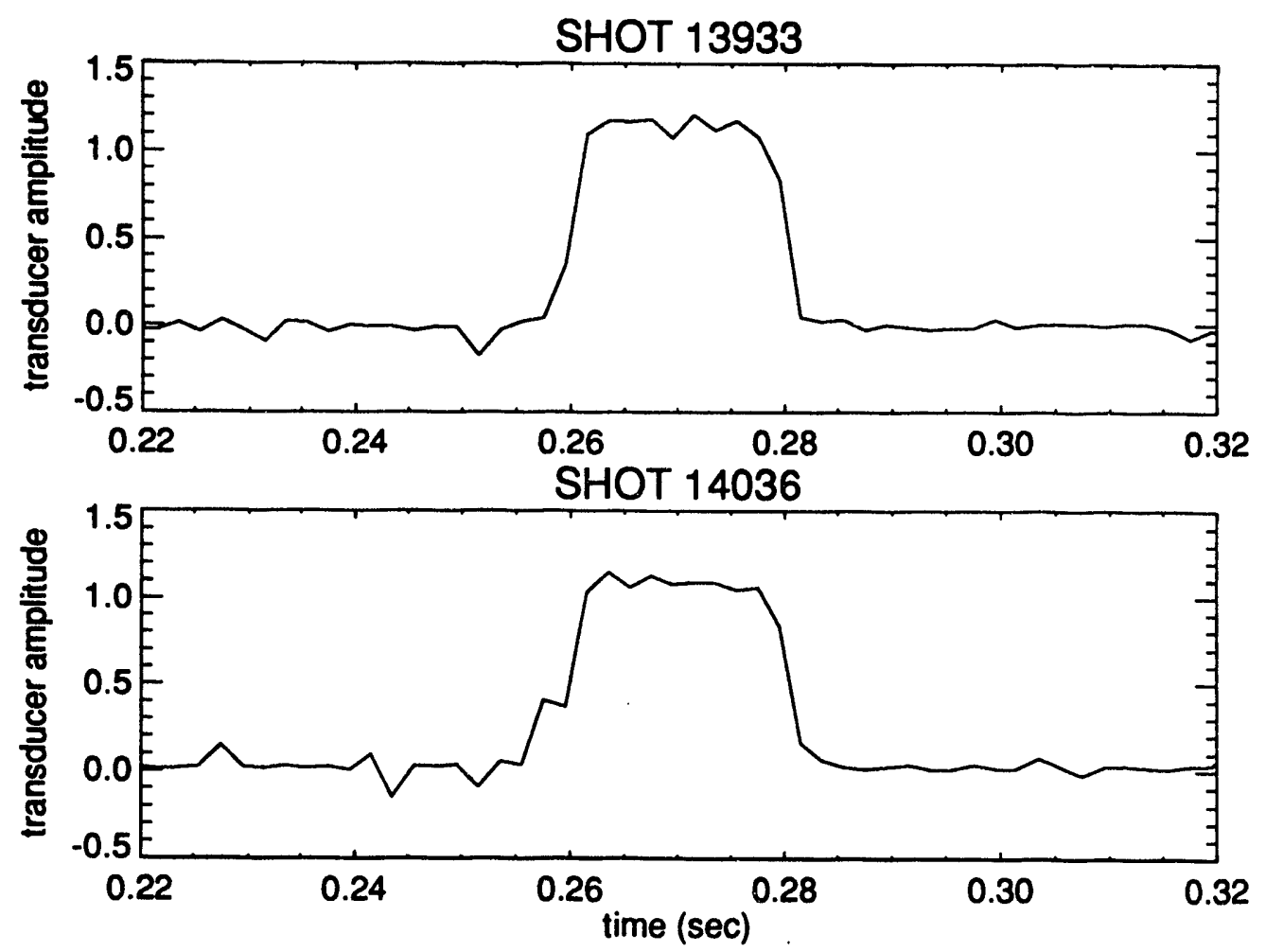

Figure 4.22: Gas valve pressure transducer signals during modulation of $285 \mathrm{kA}$ shots 1393,14036 at $\bar{n}_{e, 20}=2.9,3.3$ respectively. 


\begin{tabular}{|crrrrrr|}
\hline normalized radius & $\mathrm{n}, \mathrm{T}$ & $\mathrm{T}, \nabla \mathrm{T}$ & $\mathrm{n}, \nabla \mathrm{T}$ & $\nabla \mathrm{n}, \nabla \mathrm{T}$ & $\mathrm{T}, \nabla \mathrm{n}$ & $\mathrm{n}, \nabla \mathrm{n}$ \\
\hline .073 & -.919 & -.901 & .849 & -.054 & -.031 & -.276 \\
.189 & -.939 & -.921 & .908 & .328 & -.452 & .185 \\
.306 & -.945 & -.955 & .929 & .601 & -.579 & .391 \\
.423 & -.978 & .735 & -.779 & .043 & -.357 & .211 \\
.540 & -.989 & .960 & -.937 & -.028 & -.010 & .031 \\
.657 & -.991 & -.059 & .088 & -.144 & .548 & -.552 \\
\hline
\end{tabular}

Table 4.3: Correlation coefficients between pairs of perturbed variables.

\subsubsection{Significance of Temperature Perturbations}

The gas puffs used in these experiments produced changes in the local temperature of order $\delta T_{e} / T_{e 0} \sim .03-.10$ which are of opposite sign of the density perturbation. Electron temperatures are determined at several radii through measurements with an electron cyclotron emission polychrometer. Unlike the ECE Michelson interferometer which scans the emission spectrum every $15 \mathrm{msec}$, the polychrometer has time resolution far in excess of that required to follow temperature evolution during the density modulation transient. Unfortunately during this run not all of the polychrometer channels were reliable. Five reliable polychrometer channels were available spanning $0 \leq \mathrm{r} / \mathrm{a} \leq .65$. Signals from these channels were filtered using the same $60 \mathrm{~Hz}$ bandstop $/ 90 \mathrm{~Hz}$ low pass filter used in the analysis of the FIR data. Profiles of these signals are fit at fixed time intervals using a spline fit under tension with smoothing as described in sec. 3.4. Time histories of the local electron density and electron temperature for $\bar{n}_{e, 20}=2.2, \mathrm{I}_{p}=285 \mathrm{kA}$ are correlated at $6 \mathrm{msec}$ intervals over the period of the transient at various radii. Resulting correlation coefficients are given in table 4.3. Values of the correlation coefficients close to -1 indicate the $\tilde{n}_{e}$ and $\tilde{T}_{e}$ perturbations are highly correlated, but have the opposite sign. Correlation coefficients between all other combinations of pairs from $\tilde{n}_{e}, \nabla \tilde{n}_{e}, \tilde{T}_{e}$ and $\nabla \tilde{T}_{e}$ are also given and are systematically lower than for $\left(\tilde{n}_{e}, \tilde{T}_{e}\right)$. There is a fairly high correlation between $\tilde{T}_{e}$ and $\nabla \tilde{T}_{e}$ as well as $\nabla \tilde{T}_{e}$ and $\tilde{n}_{e}$. Correlation coefficients between $\nabla \tilde{n}_{e}$ and the other three quantities are quite a bit lower. 
These results are in line with the derivation of sec. 2.2 regarding the effect of off-diagonal terms on the values obtained for the transport coefficients. In particular, since $\tilde{T}_{e}$ is highly correlated with $\tilde{n}_{e}$, the component of perturbed flux associated with temperature is highly correlated with that component due to density perturbations. Thus the effect of the temperature perturbations appears in the perturbed convective velocity. This is as we expected since, as we found in the example of sec. 2.2 for the slow wave associated with particle transport, the density perturbation is accompanied by a temperature perturbation of opposite phase.

The density and temperature are highly correlated across the range of the profiles considered here, while the density gradient and temperature gradient are not highly correlated. This is because even though the local variations of density and temperature at a location are well correlated in time, the spatial variation of the perturbed profiles is different. In this way gradients of $\mathrm{T}_{e}$ and $\mathrm{n}_{e}$ are not well correlated.

The significance of temperature perturbations is considered further in Appendix H. Through a statistical test of an extended model it is concluded that, at least to within our ability to measure the $\mathrm{T}_{e}$ profile, we can use the model $\Gamma=-D \frac{\partial n}{\partial r}-V n$, in which the temperature effects are included implicitly, without introducing significant bias into the results. The effect of the temperature variations is manifest in the perturbed convection velocity $\mathrm{V}$. This matter is also examined in the framework of a four dimensional linear regression correlation in Appendix $\mathrm{H}$. 


\section{Chapter 5}

\section{Comparison with Theory}

Laboratory plasmas are rarely quiescent; fluctuations arise spontaneously. Gradients of local intensive plasma quantities, such as temperature and pressure, represent sources of free energy which can drive such fluctuations resulting in a state of turbulence. These fluctuations can drive fluxes of particles and energy. Broadband turbulence is observed in tokamaks. Density fluctuations in the plasma core have amplitudes of order $\tilde{\mathrm{n}} / \mathrm{n} \approx 0.01$ or less $[5,71]$. At the plasma periphery density fluctuation amplitudes are much larger with $\tilde{\mathrm{n}} / \mathrm{n} \sim 0.1-1[5]$.

On Alcator $\mathrm{C}$, density fluctuation spectra were measured with $\mathrm{CO}_{2}$ laser scattering [72]. At low densities $\left(\bar{n}_{e} \sim 1.2 \times 10^{20} \mathrm{~m}^{-3}\right)$ fluctuations of an estimated amplitude $\tilde{\mathrm{n}} / \mathrm{n} \sim 0.1$ occur in a layer approximately $5 \mathrm{~cm}$ thick and thus encompass the outermost third of the plasma. The peak value of $\tilde{n} / \mathrm{n}$ occurs at $\mathrm{r} / \mathrm{a} \sim 0.75$. This observation, also seen in Alcator $A$, is an exception to the measured turbulence characteristics in other tokamaks where $\tilde{\mathrm{n}} / \mathrm{n}$ is peaked at the plasma edge $[73,74,75,83,84,5]$. At higher densities $\left(\bar{n}_{e} \geq 2 \times 10^{20} m^{-3}\right)$ the density fluctuations on Alcator $C$ are peaked at the limiter. In the presence of Marfes there are large up-down asymmetries in the density fluctuation amplitude. Density fluctuations with an amplitude $\tilde{\mathrm{n}} / \mathrm{n} \approx 0.5$ are found in a narrow layer near the limiter. The width of that layer is smaller than the $\sim 4 \mathrm{~cm}$ resolution of the diagnostic, but was estimated to be approximately $2 \mathrm{~cm}$. Because of the limited spatial resolution of the diagnostic, properties of the density fluctuations could be studied only in the regions where they had the largest ampli- 


\begin{tabular}{|lcc|}
\hline TYPE & RESTRICTIONS & TRANSPORT PROPERTIES \\
\hline $\begin{array}{l}\text { ion temperature } \\
\text { gradient mode } \eta_{i}\end{array}$ & weak at low $\mathrm{T}$ & $\chi_{i} \gg \mathrm{D}$ \\
\hline resistive ballooning & $\mathrm{T}_{e} \leq 500 \mathrm{ev}$ & $\chi_{e}>\mathrm{D}_{e}$ \\
\hline $\begin{array}{l}\text { trapped electron } \\
\text { mode }\end{array}$ & weak at low $\mathrm{T}_{e}$ & $\mathrm{D} \gg \chi$ \\
\hline $\begin{array}{l}\text { resistivity-gradient- } \\
\text { driven turbulence } \\
\text { (w/ radiation drive) }\end{array}$ & $\mathrm{T}_{e} \leq 100 \mathrm{ev}$ & \\
\hline
\end{tabular}

Table 5.1: Characteristics of several edge turbulence models.

tudes. Either moderate or small density fluctuations can exist for $\mathrm{r} / \mathrm{a} \sim 0.7$ at the higher densities in Alcator $\mathrm{C}$, as is the case at lower density, without being apparent in this measurement since they would be obscured by the contribution from larger amplitude fluctuations $\left(\propto \mathrm{n}_{k}^{2}\right)$ associated with the Marfe.

It is logical to associate these turbulent fluctuations with anomalous transport processes in the plasma. Since the increase in the particle transport coefficients measured near the density limit is most pronounced in the outer regions of the plasma, it is reasonable to look for an explanation in terms of edge turbulence theories.

\subsection{Edge Turbulence Theories}

The outer third of the MTX plasmas in the $285 \mathrm{kA}$ density scan were characterized by relatively low temperature $\left(\mathrm{T}_{e}<250 \mathrm{ev}\right)$ and high collisionality, in the PfirschSchlüter regime. Let us now consider a variety of models which have been proposed to describe edge turbulence. None of the models has succeeded in describing all of the characteristics of edge turbulence observed on tokamaks. Certain models have had a number of successes. We now examine which models might be applicable for the high-density edge plasmas in MTX. 
Trapped electron modes are not a candidate unless $\nu_{*}<1$. Therefore they can be excluded from consideration here.

Ion temperature gradient driven turbulence $\left(\eta_{i}\right)$ can exist at any temperature but is weak at low temperatures $[85,5,86,87]$. Its characteristics include $\chi_{i} \gg \mathrm{D}$, $\chi_{e} \simeq \chi_{i}, \tilde{\phi} / T_{e} \simeq \tilde{n} / n, \tilde{T}_{i} / T_{i}>\tilde{n} / n$. This mode has been advanced to explain the enhancement in thermal conductivity in the ion channel at high densities in Alcator C. Ion thermal confinement improves in high-density Alcator $\mathrm{C}$ plasmas when peaked density profiles are formed with pellets. This could be related to the reduction in the quantity $\eta_{i}=\mathrm{d} \ln T_{i} / \mathrm{d} \ln n$, which would presumably be reduced below the critical threshold value for the instability [78].

It is difficult to reconcile the prediction $\chi_{i} \gg \mathrm{D}$ with the behavior of the global energy confinement near the density limit. Recall that essentially no significant changes were observed in $\tau_{E}$ near the density limit even as the perturbed particle transport coefficients increased dramatically in the outer region of the plasma. The diffusion coefficients essentially doubled there as the line-averaged density at $285 \mathrm{kA}$ was raised from 2.9 to $3.3 \times 10^{20} \mathrm{~m}^{-3}$. If this enhancement in D there was caused by ITG turbulence, we would expect to see an enhancement in losses in the ion thermal channel as well since, $\Delta \chi \gg \Delta D$. An enhancement in $\chi_{i}$ of this magnitude extending across such a large fraction of the plasma would be manifest as a measurable deterioration in the global energy confinement. We expect from the theory that the steady state and perturbed $\chi$ values would both increase in a similar manner.

This does not mean that ITG turbulence is not responsible for the saturation in energy confinement time at higher densities. Within the framework of the present ITG theories it does imply that the enhancement in particle transport in the outer region of the plasma near the density limit is not caused by ion temperature gradient driven turbulence.

Resistive ballooning is generally predicted to be important in regions where $\mathrm{T}_{e} \leq 500 \mathrm{ev}$ and a sufficiently large pressure gradient exists [5]. A linear theory of this model can predict a non-Boltzmann relation between density and potential fluctuations of the type which has been observed in experiment [88]. This mode will 
be considered further in a later section.

Resistivity-Gradient-Driven Turbulence - The nonlinear resistivity-gradientdriven turbulence theory was developed as an outgrowth of the linear theory of the rippling mode [89]. It should be significant only in regions of relatively low temperature. In this theory $\mathrm{D} \gg \chi$ so that energy transport induced by the mode is almost entirely due to that associated with the convected particle flux. This characteristic is consistent with the observed trend in global energy confinement in MTX near the density limit. An increase in particle transport due to this mode would not necessarily be accompanied by a decrease in global energy confinement. When the effect of radiation as a driver is included [92] a non-Boltzmann relation between potential and density $\tilde{\phi} / T_{e} \gg \ddot{n} / n$ is predicted. As noted in the preceding discussion of the resistive ballooning mode this characteristic has been observed in edge plasma turbulence. According to a summary of edge turbulence theories compiled from a meeting of the Transport Task Force workshop [99]:

"The rippling/radiation mode should only dominate during the early part of start-up, or in low-power or high-density discharges."

We will consider resistivity-gradient-driven turbulence theory further as a candidate to explain particle transport in the low temperature outer regions of high-density Alcator C plasmas.

\subsection{Resistivity-Gradient-Driven Turbulence The- ories}

The nonlinear theory of resistivity-gradient-driven turbulence considered here [89] was first derived based upon the reduced resistive magnetohydrodynamic (MHD) equations in cylindrical geometry. An overview of the published derivations of several versions of the theory is given in Appendix I. Several significant points will be summarized here. In the linear theory the rippling mode is strongly stabilized by parallel thermal conduction so that these modes are usually considered only in the context of 
the very edge of the plasma. Although the nonlinear theory was an outgrowth of the linear theory of the rippling mode, the nonlinear saturated state is radically different in character from the linear regime [89]. Owing to its different mode structure and dynamics the nonlinear state has a much weaker dependence upon the stabilizing parallel thermal diffusivity. A version of the theory was developed which included the effect of ir..- "rrity dynamics [90]. Here resistivity fluctuations are predominantly iriven by fluctuations in $\mathrm{Z}_{\text {eff }}$ so that this mode is governed by impurity ion flow along field lines proportional to $\chi_{z}=V_{t h, i}^{2} / Z^{2} \nu_{i i}$. Since $\chi_{z} \ll \chi_{\|}$the impurity gradient driven modes are significantly more robust than their temperature gradient driven analogs.

In the published results expressions for fluctuation and transport levels are derived from nonlinear theory and are in good agreement with the results of numerical calculation [90]. The turbulent particle flux is given by

$$
\Gamma_{n}=\frac{n_{0}}{L_{n}}\left(\frac{E_{0} L_{s}}{B_{z} L_{\eta}}\left(1+\eta_{z}\right)\right)^{\frac{4}{3}}\left(\chi_{z} \bar{k}_{\|}^{2}\right)^{-\frac{1}{3}}
$$

with $L_{n}^{-1}=-\frac{1}{n_{0}} \frac{d n_{0}}{d r}, \eta_{z}=L_{\eta} / L_{z}$ and $E_{0}, B_{z}$ the toroidal electric and magnetic fields respectively. Since the particle flux is linear in $\nabla n$ the equilibrium and perturbed diffusion coefficients are equal. The diffusion coefficient is

$$
D_{n}=\Gamma_{n} \frac{L_{n}}{n_{0}}=\left(\frac{E_{0} L_{s}}{B_{z} L_{\eta}}\left(1+\eta_{z}\right)\right)^{\frac{4}{3}}\left(\chi_{z} \bar{k}_{\|}^{\prime 2}\right)^{-\frac{1}{3}}
$$

which scales as

$$
D_{n} \sim T^{-5 / 6} n^{1 / 3}\left(L_{\eta}^{-1}+L_{z}^{-1}\right)^{4 / 3} L_{s}^{2} B_{z}^{-4 / 3} V_{L}^{4 / 3} r_{s}^{2 / 3} R_{0}^{4 / 3} .
$$

with $\mathrm{V}_{L}$ the loop voltage and the shear length defined as $\mathrm{L}_{s}=\mathrm{Rq} / \hat{s}$.

For typical tokamak profiles this diffusion coefficient increases with radius. It also scales as $\mathrm{D} \sim \mathrm{I}_{p}^{2} \sim \mathrm{q}^{2}$ so that particle confinement should improve with increasing current; conversely, this transport mechanism would be enhanced in plasmas with high q. Since the thermal diffusivity calculated from this model is insignificant, the thermal transport driven by RGDT is primarily associated with that carried by the convected particle flux. 
In these theories for this current driven mode, fluctuation and transport levels show a definite dependence upon the electric field strength. In experiments in TEXT, when the electri: field was reduced transiently to zero, the measured level of fluctuations was unaffected [112]. The effects of radiation as a driver were recognized and included in an extended version of the model. Two types of radiation drive terms were considered, the thermal drive [42]

$$
\gamma_{T}=-\frac{2}{3} n_{z 0} \frac{d I_{z}\left(T_{0}\right)}{d T}
$$

and the condensation drive

$$
\gamma_{T}=-\frac{2}{3} \frac{n_{z 0} I_{z}\left(T_{0}\right)}{T}
$$

with $n_{z} n_{e} I_{z}$ the radiated power density. With impurity radiation effects included, the fluctuation levels were increased with potential fluctuations emerging as larger than density perturbations $(\mathrm{e} \tilde{\phi} / T>\tilde{n} / n)$. In addition the sensitivity to the electric field could be greatly reduced [42]. These features had a greater resemblance to measured edge turbulence characteristics [99].

A similarity between edge turbulence in the TEXT tokamak and the (currentless) Advanced Toroidal Facility stellarator [113] was observed experimentally. This has been taken as evidence against the resistivity-gradient-driven turbulence model. It could be, however, than two different type of turbulence are present with and without plasma current, which happen to have similar characteristics [5].

A version of the rippling theory based upon neoclassical MHD equations [114] was developed [115]. In this theory of neoclassical resistivity-gradient-driven turbulence the rippling modes couple to the density gradient through the dependence of neoclassical resistivity on density. The density coupling coefficient to the neoclassical resistivity has a maximum value around $\nu_{* e} \sim 1$ and vanishes in the limits $\nu_{* e} \rightarrow 0$ or $\nu_{* e} \rightarrow \infty$. The equilibration of density fluctuations is affected by collisions between trapped and untrapped particles. This has a much weaker stabilizing effect than thermal conduction. Therefore the region where resistivity-gradient-driven turbulence is significantly excited broadens inward from the edge of the plasma to the region where $\nu_{* e} \leq 1[115]$. 
The latter assertion is rather significant. With conventional rippling modes the resistivity response is strongly damped by parallel thermal conduction [117]. Thus conventional rippling modes are not thought to be an important instability mechanism in regions of moderate $\chi_{\|}$. The turbulence in the nonlinear saturated theory considered has a vastly different structure which makes it robust, even in a relatively high temperature regime.

In the conventional picture a linearly unstable rippling mode [118] grows, evolving into saturated resistivity-gradient-driven turbulence [115, 89]. Analysis of the linear instability in the collisional regime was published by Rogister [119], who included the influence of diamagnetic drifts, ion viscosity and the effect of toroidal curvature. He assumed the linear growth rate was small compared to the electron diamagnetic frequency. Results indicated that toroidal curvature acts to destabilize the mode in a particular regime.

Hassam and Drake [120] analyzed the linear stability of the rippling mode at lower collisionalities using the two-fluid Braginskii equations. Once the complete electron physics was included, it was found that the conventional rippling instability could become linearly stable at rather low temperatures in a sheared slab system.

Tang et al. [121] derived equations for use in the study of resistive electrostatic instabilities. These included the effect of impurity gradients and could be used in both the semicollisional and strongly collisional regimes. Linear stability analysis was performed in a sheared slab limit containing an approximate treatment of toroidal curvature effects. Results of the linear calculation in the semicollisional regime indicate that toroidal curvature effects are not sufficient to overcome the stabilizing parallel electron dynamics. Also only $\mathrm{Z}_{\text {eff }}$ profiles with large gradients in the outward radial direction $\left(\eta_{z}<-1\right)$ have a significant impact on the instability threshold. In the latter case $\left(\eta_{z}=-1\right)$ the rippling modes are linearly stable for typical tokamak parameters for $\mathrm{T}_{e} \tilde{>} 50 \mathrm{ev}$.

In the region of the $285 \mathrm{kA}$ shots where an enhancement in the particle transport coefficients was measured near the limit density $(0.6 \leq \mathrm{r} / \mathrm{a} \leq 0.8)$ the measured electron temperatures at $255 \mathrm{msec}$ varied from $\sim 60 \mathrm{ev}$ to $\sim 250 \mathrm{ev}$. The $Z_{\text {eff }}$ profile is 
nearly spatially constant in Alcator C [122]. Thus the linear theory of Tang et al., which includes complete electron physics, impurity gradient effects and the approximate effect of toroidal curvature, predicts the rippling mode is linearly stable in the aforementioned region of these plasmas.

The turbulence in the nonlinear theory has a structure rather different from that in the linear theory and is much more robust. Diamond, Hahm and Kwon argue as follows [115]:

“... both theoretical and computational analyses $[89,90]$ have demonstrated that the nonlinear evolution of RGDT is characterized by the nonlinear broadening of an asymmetric mode structure, so that the resistivity and potential fluctuations move away from the rational surface and decouple from the current perturbation. This current decoupling drastically modifies the usual linear structure [118] of the perturbation and renders the stabilizing influence of field line bending ineffective. It is also crucial to note that the tokamak edge is rarely quiescent $[124,75,83,97,96]$, because of the presence of impurities, plasma wall interaction, etc. Therefore the conventional picture of linear instability developing from a quiescent plasma at equilibrium is not necessarily appropriate. Hence the tokamak edge fluctuations can only be understood by nonlinear theory."

They further assert that since rippling modes are nondispersive, diamagnetic effects are negligible in comparison to the nonlinear decorrelation rate at saturation [115].

"Thus NRGDT is always in the strong turbulence regime and the real frequency and $\omega_{* e}$ effects play no role."

An important assumption in the rippling theories is that the perturbed pressure induced by fluctuations is zero, due to parallel sound wave propagation . Parallel pressure balance occurs when $k_{\|}$is sufficiently large so that $\bar{\omega}<k_{\|} c_{s}$. Here $\bar{\omega}$ is the fluctuation decorrelation rate, $\mathrm{c}_{s}$ is the sound speed and $k_{\|}=k_{\|}^{\prime} x=m x / L_{s} r_{s}$. It was recognized that the accoustic zone, where parallel pressure balance is satisfied, is usually more than an order of magnitude further out on the eigenmode than the radiation 
zone, which ends where the radiation drive $\gamma_{n}$ is balanced by parallel thermal conduction $\sim \chi_{T} k_{\|}^{\prime 2}$ [42]. Since the acoustic zone and the radiation zones do not overlap, the condensation effect (driven by $\gamma_{n}$ ) does not survive. Earlier results derived including the condensation drive and assuming a pressure balance existed, showed that the radiation drive enhancement was most pronounced at the lowest temperature. A separate analysis which contained no parallel pressure balance constraint a priori was performed to determine the enhancement of the turbulence level due to impurity radiation drive over that due to the resistivity gradient drive alone. A transcendental expression for the turbulent radial velocity enhancement factor $\left(\mathrm{F} \equiv \tilde{V}_{r} / V_{r 0}\right)$ was derived, where $V_{r 0}=\frac{E_{0} L_{Q}}{B_{z} L_{\eta}}=\frac{3 E_{0} L_{Q}}{2 B_{z} L_{T}}$. The expression is [42]

$$
\begin{aligned}
F\left[1+\frac{2}{3}\left(\frac{\chi_{n}}{\chi_{T}}\right)^{2 / 3}-\frac{\Gamma_{T}}{\Gamma_{n v}}\right]= & \left(1-\frac{2}{3} \eta_{n}-\eta_{n} \frac{\Gamma_{n}}{\Gamma_{n v}}\right)+\left(\Gamma_{T}+\left(\frac{\chi_{n}}{\chi_{T}}\right)^{2 / 3} \Gamma_{n}\right) F^{1 / 3} \\
& +\frac{1}{\Gamma_{n v}}\left(F^{2 / 3}-F^{5 / 3}\right)
\end{aligned}
$$

with $\gamma_{j}=\Gamma_{j} V_{r 0}^{-2 / 3}\left(\chi_{j} k_{\|}^{\prime 2}\right)^{-1 / 3}, \mathrm{j}=\mathrm{T}, \mathrm{n}$, and $\Gamma_{n v}=\left(V_{i} k_{\|}^{\prime} V_{r 0} \Gamma_{T} \Gamma_{n} / \gamma_{T} \gamma_{n}\right)^{2}$. The usual ordering for a tokamak plasma is $\chi_{T} / \chi_{n} \sim 10^{3}$ and $\Gamma_{n v} \gg \Gamma_{n} \gg \Gamma_{T}$. Note that for the case $\gamma_{T} \sim \gamma_{n}$ the second term on the right hand side of eq. 5.6 reduces to $\approx\left(\Gamma_{T}+\left(\frac{\chi_{n}}{\chi_{T}}\right)^{1 / 3} \Gamma_{T}\right)$. As a consequence the condensation contribution is smaller than that due to the thermal instability contribution by an order of magnitude.

We now examine the relative importance of the enhancement due to radiation for a portion of the collisional MTX outer plasma. Specifically we take parameters typical of the high-density $285 \mathrm{kA}$ shots near the outer edge of the region where transport coefficients were obtained, such as $\mathrm{n}_{e}=2 \times 10^{20} \mathrm{~m}^{-3}, \mathrm{~T}_{e} \simeq 150 \mathrm{ev}, \mathrm{B}_{T}=6.5 \mathrm{~T}, \mathrm{~V}_{L}=$ $2 \mathrm{v}, \mathrm{L}_{s}=1.2 \mathrm{~m}, \mathrm{~m}=22, \mathrm{~L}_{T}=.05 \mathrm{~m}$ and $\eta_{n} \sim \frac{1}{3}$. We use a realistic value of $Z_{\text {eff }}=1.2$ and a radiation power density typical for the outer region of a high-density Alcator $\mathrm{C}$ plasma of $1 \mathrm{~W} / \mathrm{cm}^{-3}$ [125]. The rms poloidal mode number $\mathrm{m}$ was as assumed in reference [92] for Alcator and is similar to the value inferred from $\mathrm{CO}_{2}$ scattering measurements [72]. We also assume here that $\gamma_{T} \approx \gamma_{n}$. For a single carbon impurity one obtains $\chi_{T} / \chi_{n} \approx 800, \Gamma_{T}=.01, \Gamma_{n}=.09$ and $\Gamma_{n v}=5.1$. For these parameters the expression for the turbulent radial velocity enhancement factor reducest to $\mathrm{F} \approx 1$. Thus we see that in the region where $T_{e} \sim 150 \mathrm{ev}$ the enhancement of the turbulence 
level due to radiation predicted by this theory is not significant. The derivation of reference [92] corresponds to the unphysical situation of $\chi_{T} \simeq \chi_{n}$. Even in this case, where the condensation drive is not reduced, the result would be the same.

At much lower temperatures $T_{e} \sim 15$ ev the theory would predict a substantial enhancement of turbulence level due to radiation. As one moves to regions of higher temperature both the current density $\mathbf{J}$ and the parallel diffusivity $\chi$ increase. Since the radiated power density determined from the bolometry measurements typically varies slowly with radius in Alcator $C$ [125] the condensation radiation drive term $\gamma_{n} \sim$ $I_{o} / T_{e}$ decreases with increasing temperature. For coronal equilibrium the thermal drive contribution $\left(\gamma_{T} \sim-\frac{2}{3} n_{z 0} \frac{d I_{Z}\left(T_{0}\right)}{d T}\right)$ for low $\mathrm{Z}$ impurities, such as carbon and oxygen would also decrease rapidly as the electron temperature is raised from 15 to $150 \mathrm{ev}$ [101]. In fact in coronal equilibrium over portions of this range $\frac{d I_{z}}{d T}>0$ for these impurities, resulting in a negative contribution to $\gamma_{T}$.

\subsubsection{Comparison of Transport Coefficients with RGDT Theory}

Since the nonlinearly saturated state of resistivity-gradient-driven turbulence is much more robust than the linear theory predicts we can consider applying it to a plasma with a temperature in the $100 \mathrm{ev}$ range [102]. Large amplitude density fluctuations have been observed in the outer third of the Alcator C plasma, so that it is scarcely quiescent. We will consider applying the nonlinear theory to the outer third of the plasmas of the $285 \mathrm{kA}$ density scan where the temperature is moderately low ( $\mathrm{T}_{e} \leq 250 \mathrm{ev}$.) Neoclassical rippling modes would be excited in a region extending to where $\nu_{* e} \leq 1$ [115]. For the shots of the $285 \mathrm{kA}$ density scan the plasma in the region

- considered here $(0.6 \leq r \leq 0.8)$ is mostly in the Pfirsch-Schlüter regime of collisionality with $\nu_{* e}$ ranging from $\sim 5$ to over 100 and increasing rapidly with radius.

As shown in the previous section the enhancement due to impurity radiation predicted by the theory is insignificant in this region. Thus the version of the theory which includes resistivity gradient drive with impurity dynamics is the most relevant 


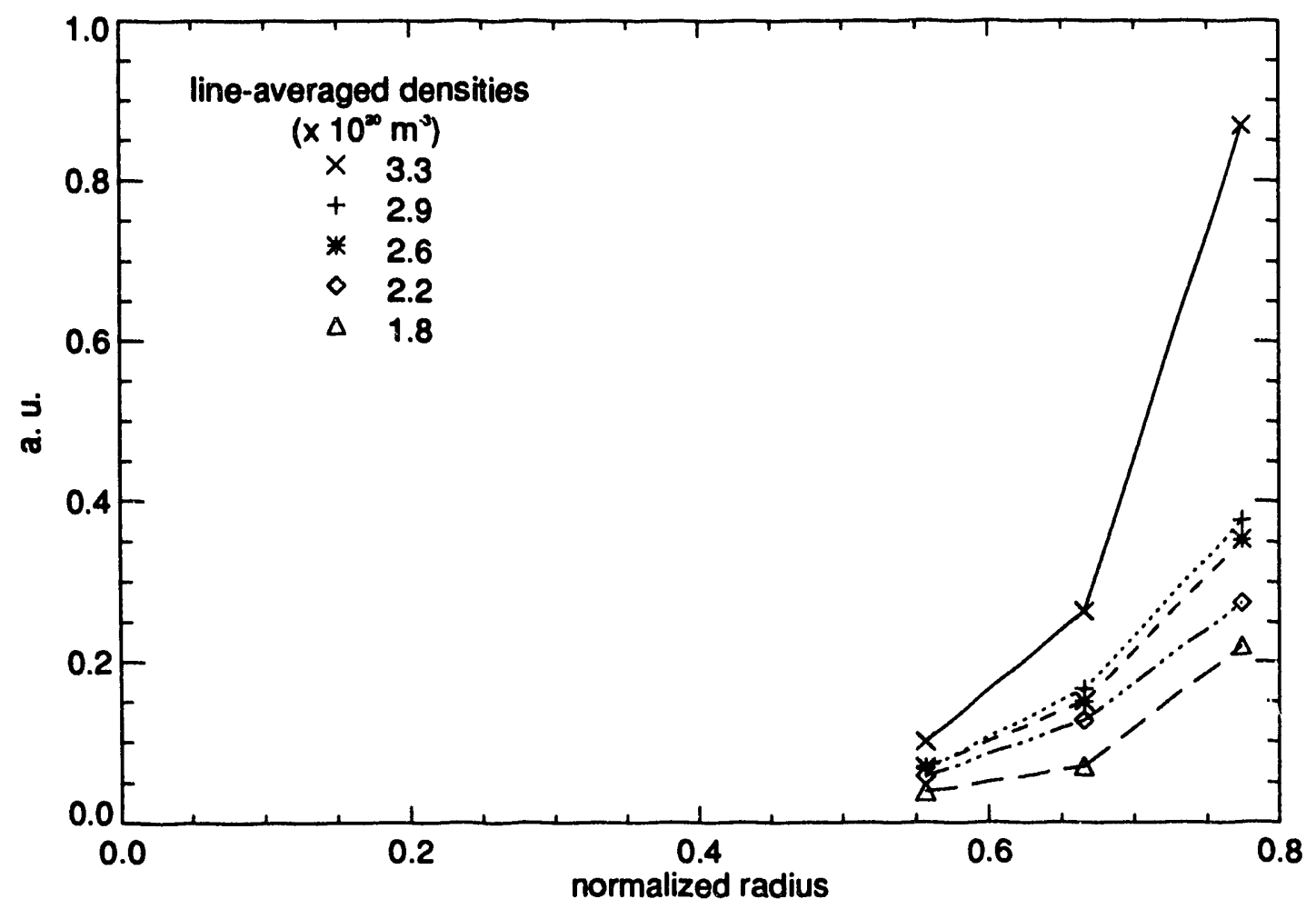

Figure 5.1: Scaling of resistivity gradient driven turbulence diffusion coefficients for plasmas of the $285 \mathrm{kA}$ density scan.

for this collisional portion of the MTX plasma. The diffusion coefficient given for this case, eq. 5.2, is independent of the density gradient. Therefore the perturbed diffusion coefficient reduces simply to the equilibrium diffusion coefficient.

The scaling of $\mathrm{D}$ given in eq. 5.3 is evaluated using data from shots at the five densities $\bar{n}_{e, 20}=1.8,2.2,2.6,2.9,3.3$ with $\mathrm{I}_{p}=285 \mathrm{kA}$ using $\eta_{z}=0$. Results in fig. 5.1 show a trend in the profiles of D similar to that in the experimentally determined values. In particular there is a large increase in $\mathrm{D}$ at $\bar{n}_{e, 20}=3.3$ near the density limit. Most of the increase in the calculated values of $\mathrm{D}$ across this scan is due to decreases in $\mathrm{T}_{e}, \mathrm{~L}_{T e}$ and to a lesser extent to an increase in the loop voltage.

Reasonable values of Alcator $\mathrm{C}$ and TEXT edge plasma parameters are given in table I of reference [92] and are used in the calculation of $\left(D_{n, I_{z}=0}\right)$, the diffusion coefficient in the absence of enhancement due to radiation, given in table III of reference [92]. We examine the assumptions used in that calculation. First the value of $\chi_{Z}$ was calculated by setting the value of $Z$ in the formula equal to $Z_{e f f}$. An rms 


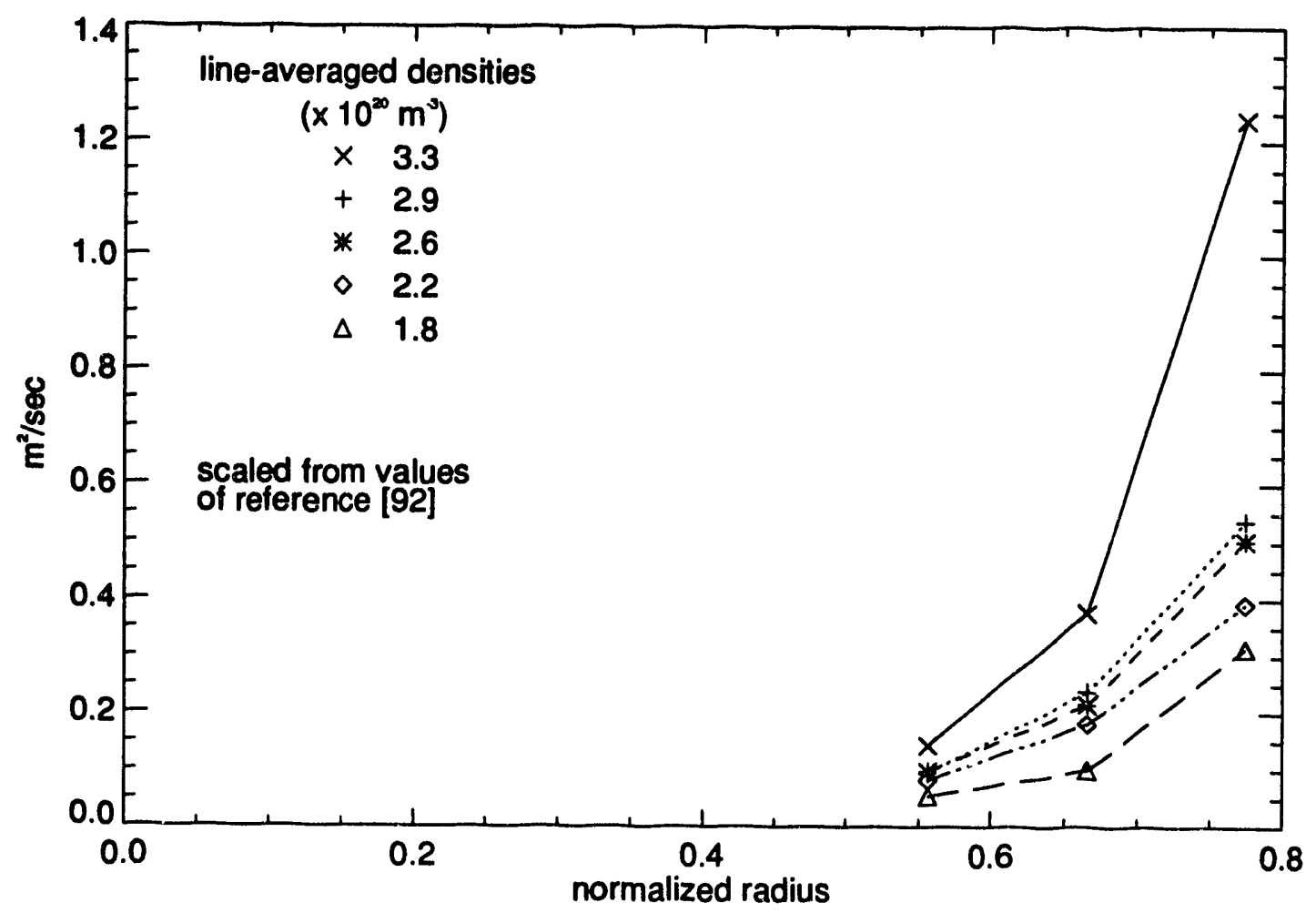

Figure 5.2: RGDT diffusion coefficient values scaled, according to assumptions, from values in ref. 92 .

poloidal mode number $\mathrm{m}=22$ was used for Alcator $\mathrm{C}$, which is reasonably close to the value determined from measurements [72]. The loop voltage is not specified, but a typical value for TEXT would be $\sim 2 \mathrm{~V}$. The value of loop voltage deduced from the derived parameters for TEXT of table II is $50 \mathrm{v}$. The discrepancy between these two values is similar to the conversion factor (29.98) which relates the mks and Gaussian values of $\mathrm{J}_{z 0} / \mathrm{B}_{z}$, i.e.

$$
\left(\frac{J}{B}\right)_{\text {Gaussian }} \Rightarrow \frac{1}{4 \pi \epsilon_{0} c}\left(\frac{J}{B}\right)_{m k s} .
$$

If the value for $\mathrm{D}_{n, I_{z}=0}$ for Alcator $\mathrm{C}$ from table III of reference [92] is scaled using this conversion factor then the coefficient values of fig. 5.2 are obtained. Like the value for $\mathrm{D}_{n, I_{z}=0}$ in table III of reference [92] these values are similar in magnitude to the values determined experimentally. But since the formula for the rms fluctuation velocity is dimensionally correct in MKS units, use of the conversion factor is not appropriate. In reference [92] the values of $\mu_{0} J_{z 0} / B_{z}$ specified in table II of ref. [92] correspond to anomalously large values of the loop voltage in mks units when related 
by Spitzer resistivity.

The significant result is that the scaling for $\mathrm{D}$ given by eq. 5.3 compares well with measured values of diffusion coefficient in this collisional plasma. Particle transport coefficients are available from a limited number of gas modulation shots at lower densities. These plasmas are in the linear, neo-Alcator regime of energy confinement and their temperatures in the region $0.6 \leq \mathrm{r} / \mathrm{a} \leq 0.8$ are substantially higher than for the high-density shots previously considered. This is especially true for the higher current/lower q shots which have wider electron temperature profiles. On those shots the electron temperature in the aforementioned region varies from $\sim 200$ to $\sim 450$ ev. Also the plasma in this region of these discharges is generally in the bananaplateau regime of collisionality. The scaling of eq. 5.3 does not compare nearly as well with the empirical diffusion coefficients in these cases. This is plausible since the theory was derived using the equations of resistive MHD, valid only in the collisional regime. A study of edge turbulence fluctuation levels in the Caltech tokamak showed no dependence on the local collisionality over a range of nearly 100 [75]. But the values of $\nu_{* e}$ at $\mathrm{r} / \mathrm{a}=0.9$ varied from 0.2 to $\sim 10$ in that study so that the collisional regime was not explored. Although the nonlinear theory could apply in the $100 \mathrm{ev}$ temperature range, we should not expect it to apply in regions of electron temperature in the 500 ev range.

\subsubsection{Comparison with Trends in Global Energy Confine- ment Time}

Figure 5.3 shows the integrated power balance from ONETWO analysis of shot 14079, one of the shots in the $285 \mathrm{kA}$ density scan. The particle source term is calculated assuming a global particle confinement time of $3 \mathrm{msec}$, estimated using data from DENSEPACK measurements on Alcator $\mathrm{C}$ as was described in sec. 3.5. This value of $\tau_{p}$ is rather smaller than the crude estimate obtained from the single $\mathrm{H}_{\alpha}$ detector. Radiated power profiles were not available for this shot. In a typical high-density shot in Alcator $\mathrm{C}$ the radiated power in the core plasma, excluding that from the Marfe at the edge, is a low fraction of the total input power, between 10 and 30 percent. 

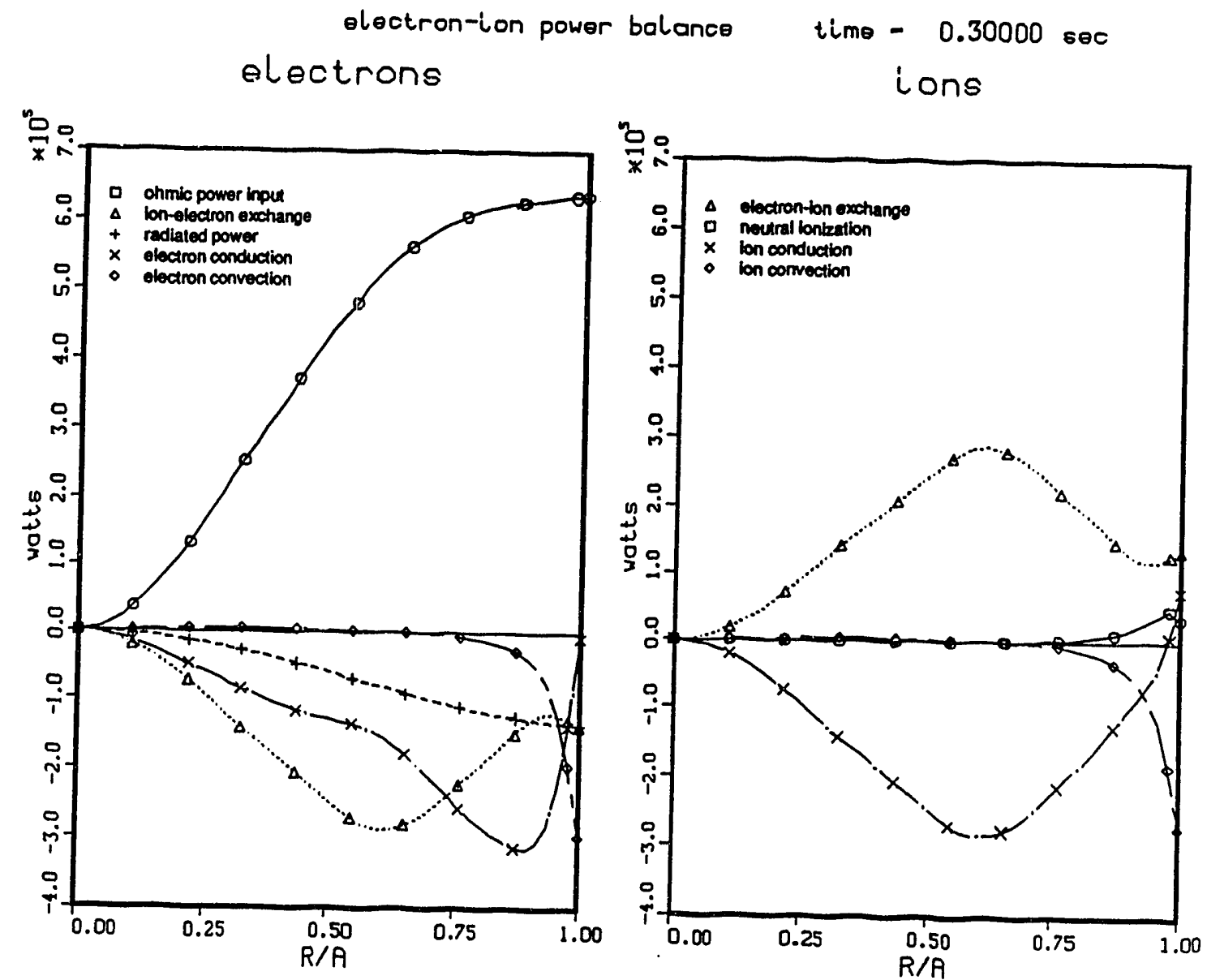

Figure 5.3: Steady state power flux calculated by ONETWO. 
This is consistent with measurements from Alcator C at MIT [125]. At line-averaged densities $\bar{n}_{e} \geq 1.5 \times 10^{20} \mathrm{~m}^{-3}$ the measured $\mathrm{Z}_{e f f}$ approaches 1 . A typical profile of the radiated power density from a previous high-density MTX plasma, having values much like the values given from measurements on Alcator $\mathrm{C}$ [125], are input into the code. The convected energy flux, due to the steady state flux of particles, is seen to be small compared to the conducted and radiated power flux except at the very edge of the plasma. As $\bar{n}_{e, 20}$ was increased to 3.3 in the $285 \mathrm{kA}$ density scan the perturbed diffusion coefficients increased by a factor of $\sim 2$ relative to the values at lower densities in the saturated confinement regime. If the particle flux was to be increased by a similar factor the proportional increase in the convected energy flux would not substantially increase the total energy flux except at the very edge. The energy confinement is already poor at the plasma edge, so that the global energy confinement time is influenced largely by energy transport in the region of good confinement, $\mathrm{r}_{q=1}<\mathrm{r}<r_{q=2}$, where the convected energy flux is negligible. Thus an increase in the steady state convected energy transport by a factor of 2 would not be reflected as a significant change in the global energy confinement time.

As shown in fig. 4.12 the global energy cunfinement time determined by the diamaginetic luop shows no substantial deterioration at $\bar{n}_{e, 20}=3.3$, even though the measured perturbed particle transport coefficients are nearly doubled in the outer plasma compared to the values at $\bar{n}_{e, 20}=2.9$. The values of $\tau_{e}$ determined by the DML agreed well with values calculated using ONETWO analysis as was shown in table 4.2. This observation is thus consistent with turbulent particle transport being enhanced by a type of turbulence, such as resistivity-gradient-driven turbulence, which induces a negligible increase in the conducted thermal flux. Some other mechanism would need to be inferred to explain conducted conducted energy fluxes in both the ion and electron channels in this region of the plasma.

The density profile maintains a similar shape through this scan, even near the density limit. This can be understood through the simultaneous increases in the perturbed diffusion and convection coefficients. These observations imply that the net steady state particle flux may not behave in the same manner as the perturbed 
particle transport coefficients.

\subsubsection{Tests of Assumptions Used in Reduced Braginskii Equations}

The outer regions of these high-density Alcator $\mathrm{C}$ plasmas meet the criteria used in deriving the reduced nonlinear Braginskii fluid equations. These equations are similar to the equations of resistive MHD, used to derive the resistivity-gradientdriven turbulence theory. These criteria are

$$
\begin{aligned}
\rho_{s}^{2} \nabla_{\perp}^{2} & \ll 1, \\
\frac{\partial}{\partial t} & \ll \nu_{e i}, \\
\nu_{e}^{*} & \gg\left(\epsilon^{-3 / 2}\right)^{2}, \\
\frac{\partial}{\partial t} & \ll \tau_{A l v e ́ n}^{-1}, \text { and } \\
\nabla_{\perp} & \gg a^{-1} .
\end{aligned}
$$

The conditions of equations 5.11, 5.12 are simply orderings appropriate for resistive modes. Equation 5.10 is equivalent to stating that resistive MHD is valid only in the collisional regime $\left(\frac{V_{e}^{2}}{\nu_{\text {ei }}^{2}} \ll R_{0}^{2} q^{2}\right)$ where trapped particle effects are insignificant [114].

\subsubsection{Vorticity Decoupling Approximation}

An important approximation in the RGDT theory is the decoupling of the vorticity equation from the other equations describing evolution of the turbulence. Turbulent fluctuations at saturation are characterized in the theory by mixing lengths $\Delta_{i}^{k}$. Because $\chi_{Z} \ll \chi_{T}$ the theory predicts that the density fluctuation mixing length $\Delta^{z}$ is much greater than the temperature fluctuation mixing length $\Delta^{T}$. Since the radial scale of the $T_{e}$ fluctuation mixing length is the smallest, we will assume that the mode structure of the $T_{e}$ perturbation determines the limit on the applicability of the $\mathrm{J}_{z}=0$ approximation in cylindrical coordinates. In this case the validity of the approximation can be examined using the derivation presented in the appendix of 
reference [89]. A scaling argument is presented there which shows that the magnitude of the terms ignored by setting $\tilde{J}=0$ is negligibly small compared to others in the RGDT equations if

$$
W^{2} \gg \frac{\chi \| \eta_{0} \rho_{m}}{B_{z}^{2}}
$$

where $W$ the mode radial width, $\eta_{0}$ the Spitzer resistivity, $\rho_{m}$ the mass density, $\mathbf{B}_{z}$ the toroidal field and $\chi_{\|}$electron therrnal diffusivity. Taking $\Delta_{T}=\mathrm{W}$, with

$$
\Delta_{T}=\left(\frac{L_{0} E_{0}}{L_{\eta} B_{z}}\right)^{1 / 3}\left(\chi_{\|} k_{\|}^{\prime 2}\right)^{-1 / 3}
$$

as given in reference [89], we obtain an inequality which scales as

$$
\frac{L_{s}^{2} E_{0}^{2 / 3}}{L_{\eta}^{2 / 3}} \frac{B^{4 / 3} n^{2 / 3}}{T^{8 / 3} k_{y}^{4 / 3}} \gg 1 .
$$

It is clear that the equality is more well satisfied for high density and high field when other parameters are held fixed. The $1 / e$ point in the scattered power intensity $\mathrm{S}(\mathrm{k})$ measured for Alcator $\mathrm{C}$ is at $\bar{k}=15 \mathrm{~cm}^{-1}=2 \pi / \lambda$ for both low and high density [72]. The turbulence is isotropic in the poloidal-radial plane. This value of $\bar{k}$ would correspond to an rms mode number of $\mathrm{m} \sim 30$. The inequality of equation 5.15 is marginally satisfied for values of $\mathrm{m} \leq 70$ for the parameters of the $285 \mathrm{kA}$ shots with $\mathrm{T}_{e}=200 \mathrm{ev}$, and is well satisfied in that case for $\mathrm{m} \leq 30$. For $\mathrm{T}_{e}=100 \mathrm{ev}$ the inequality is satisfied well for $\mathrm{m} \leq 80$.

The TEXT tokamak has edge turbulence characterized an rms poloidal mode number of $\bar{m} \approx 50$ [5]. The temperature profile on a typical TEXT shot, at similar $\mathrm{q}$, is roughly similar to that on Alcator $\mathrm{C}, \mathrm{i}$. e. central $\mathrm{T}_{e} \sim 1 \mathrm{keV}, \mathrm{T}_{\text {edge }} \sim 15$ $e V$. We can realistically assume values of $\mathrm{L}_{s}, \mathrm{E}_{0}, \mathrm{~L}_{\eta}$ on TEXT are roughly similar to those in Alcator $\mathrm{C}$. The edge density $(\mathrm{r} / \mathrm{a} \sim 0.9)$ in TEXT is rather smaller than for the higher density Alcator C plasma $\left(\bar{n}_{e} \sim 8 \times 10^{18}\right.$ vs. $\left.2 \times 10^{20} \mathrm{~m}^{-3}\right)$ and the field is typically $2 \mathrm{~T}$ versus $6.5 \mathrm{~T}$ for Alcator $\mathrm{C}$ in these shots. Thus in a location in the outer plasma at the same temperature the above inequality is more well satisfied on Alcator $\mathrm{C}$ than on TEXT by a factor of order 10 due to the higher density and field strength. 
The inequality above is more well satisfied for the high-density, high-field Alcator $\mathrm{C}$ edge plasmas than for those in tokamaks with more typical lower densities or much higher edge temperatures. This inequality is also satisfied for modes of interest in the warmer regions $\left(\mathrm{T}_{e}<250 \mathrm{ev}\right)$ where the theory was applied in the previous section. Another important difference between the plasma in Alcator $\mathrm{C}$ in the region $0.6 \leq \mathrm{r} / \mathrm{a}$ $\leq 0.8$ and the edge plasma in most other machines at $r / a \sim 0.9$ is that the radiation drive term is predicted to be insignificant in the former case by the theory.

The RGDT theory was derived in the cylindrical approximation. When toroidal curvature is included to first order the correction to the vorticity equation becomes [93]

$$
\rho \frac{d}{d t} \nabla_{\perp}^{2} U=-B_{z} \nabla_{\|} J_{\|}+\frac{2}{R_{0}} \frac{\partial P}{\partial y}
$$

with $\mathrm{R}_{\mathbf{0}}$ the tokamak major radius and $\mathrm{y}$ the coordinate along the vertical axis of the torus. The third term adds a new mechanism for coupling density and temperature fluctuations to the vorticity dynamics. The curvature term is a driver in the g-mode or resistive ballooning mode. This "separate" driving term is excluded from consideration in the analysis of the rippling mode, in part to simplify the equations [103]. While there can be conditions under which the toroidal curvature can be neglected, scaling estimates indicate this is probably not a good approximation for the Alcator $\mathrm{C}$ plasma considered. The vorticity decoupling approximation probably oversimplifies the actual dynamics of the turbulence in the Alcator $\mathrm{C}$ plasma.

\subsubsection{Other Notes about the RGDT Theory}

Density and temperature fluctuations were measured in the bulk plasma side of the velocity-shear location of the TJ-1 tokamak using a fast swept Langmuir probe technique [104]. Temperature fluctuations were observed having a fractional amplitude close to that of the density fluctuations. They were mutually correlated with the two being $\sim \pi$ radians out of phase. This observation $\left(\frac{\tilde{n}}{n} \approx-\frac{\tilde{T}}{T}\right)$ suggests that the condensation drive mechanism, invoked with the assumption of constant pressure in ref. [92], could play a role in determining edge fluctuation levels. This would appear to be consistent with the constant pressure assumption used in the rippling mode 
theories. The difficulty with the rippling theory is that the temperature perturbation has a mixing length much smaller than that of the density perturbation. It is localized to a narrow region near the rational surface where parallel sound wave propagation is typically unable to maintain constant pressure. The density and temperature fluctuations in general have different amplitudes in the theory. Thus the rippling mode lacks the right structure to create such a condensation radiation drive.

The version of the theory driven by impurity dynamics, without impurity radiation effects, also uses a pressure balance assumption over the larger mode structures of the density fluctuations. In this case, however, the pressure balance is enforced through a relation between the perturbation densities of the hydrogenic and impurity ions and the electrons. The relation for the ionic and single impurity species is

$$
2 \tilde{n}_{i}+(1+Z) \tilde{n}_{I}=0
$$

The equation used to describe impurity dynamics in the RGDT model was derived using the assumptions $n_{I} Z \ll n_{i}$ and $Z_{\text {eff }} \simeq 2$. The approximate form used is valid if in the impurity ion parallel momentum equation

$$
n_{I} m_{I} \frac{\partial}{\partial t} v_{\|, I}=-\nabla P_{I}+n_{I} Z e E_{\|}-n_{e} e\left(Z_{e f f}-1\right) \eta_{s p} J_{s p}+n_{i} m_{i} v_{i i}\left(Z_{e f f}-1\right)\left(v_{\|, i}-v_{\|, I}\right)
$$

terms ( $\mathrm{i}$ and $\mathrm{iv}$ ) dominate indicating that the impurity dynamics is dominated by a balance of the parallel gradient of impurity pressure with the impurity-hydrogenic ion friction. Even if $Z_{\text {eff }}-1 \ll 1$ these terms can still dominate in a regime of sufficiently high collisionality, i. e. $\nu_{i i}$ large. In the high-density MTX plasmas considered in the $285 \mathrm{kA}$ density scan $Z_{\text {eff }}$ is believed to fall in the range $1<Z_{\text {eff }}<1.2$ and be approximately spatially constant. The most obvious difficulty with the above assumptions for these Alcator $\mathrm{C}$ plasmas is that for typical low $\mathrm{Z}$ impurities present in Alcator $\mathrm{C}$, such as carbon, oxygen, the impurity ion density is not large enough to satisfy equation 5.17 for realistic magnitudes of density fluctuations. Also the theory doesn't explain the poloidal asymmetries in the edge density fluctuations. 


\subsection{Resistive Ballooning Theory}

In a more recent paper the linear stability of drift resistive ballooning modes in tokamak edge plasmas is studied numerically [88]. The theory is based upon the reduced Braginskii equations which, as shown previously, apply to the region of the MTX plasmas under consideration. A broad range of poloidal mode numbers was found to be unstable for edge plasma parameters of TEXT and DIII-D. As the value of $\eta_{e}\left(=\mathrm{d} \ln T_{e} / \mathrm{d} \ln n\right)$ is increased a greater range of long-wavelength modes is stabilized. For TEXT with $\eta_{e}=0$ the low $\mathrm{m}$ stability threshold is $\mathrm{m}=4$, but for $\eta_{e}=1$ this threshold increases to $m=25$. In the outer regions of the high-density MTX shots considered $\eta_{e} \sim 2-4$. If this was to cause a broad range of low $m$ modes to be linearly stable, it would be difficult to reconcile the predictions of the linear theory with the measured spectrum of the turbulent edge density fluctuations. These show the Alcator $\mathrm{C}$ edge turbulence is essentially isotropic in the $r, \theta$ plane with a gaussian shape to the density fluctuation autocorrelation spectrum centered at $k=0$, with an rms mode number with near 30 . The theory does predict a non-Boltzmann relation between density and potential fluctuations characteristic of tokamak edge turbulence.

Using a mixing length argument the particle diffusion coefficient was estimated as $\mathrm{D} \sim \gamma / k^{2}$, where $\gamma$ is the linear growth rate and $\mathrm{k}$ is the perpendicular wave vector [88]. The result given is

$$
D=(2 \pi q)^{2} \rho_{e}^{2} \nu_{e i}\left(R / L_{n}\right)
$$

which is seen to be essentially the neoclassical transport coefficient enhanced by a factor $R / L_{n}$. Because the diffusion coefficient $D$ is proportional to the gradient of density, through $\mathrm{L}_{n}$, the perturbed diffusion coefficient is equal to twice $\mathrm{D}_{e q}$.

It is interesting to note some similarities in the scaling of $D_{R B}$

$$
D_{R B} \sim T^{-1 / 2} n q^{2} B^{-2} R L_{n}^{-1}
$$

with that from the nonlinear RGDT theory

$$
D_{R G D T} \sim T^{-5 / 6} n^{1 / 3} q^{2} B^{-4 / 3} R^{2 / 3} r_{s}^{2 / 3} L_{\eta}^{-4 / 3} \hat{s}^{-2} V_{L}^{4 / 3}\left(1+\eta_{z}\right)^{4 / 3}
$$




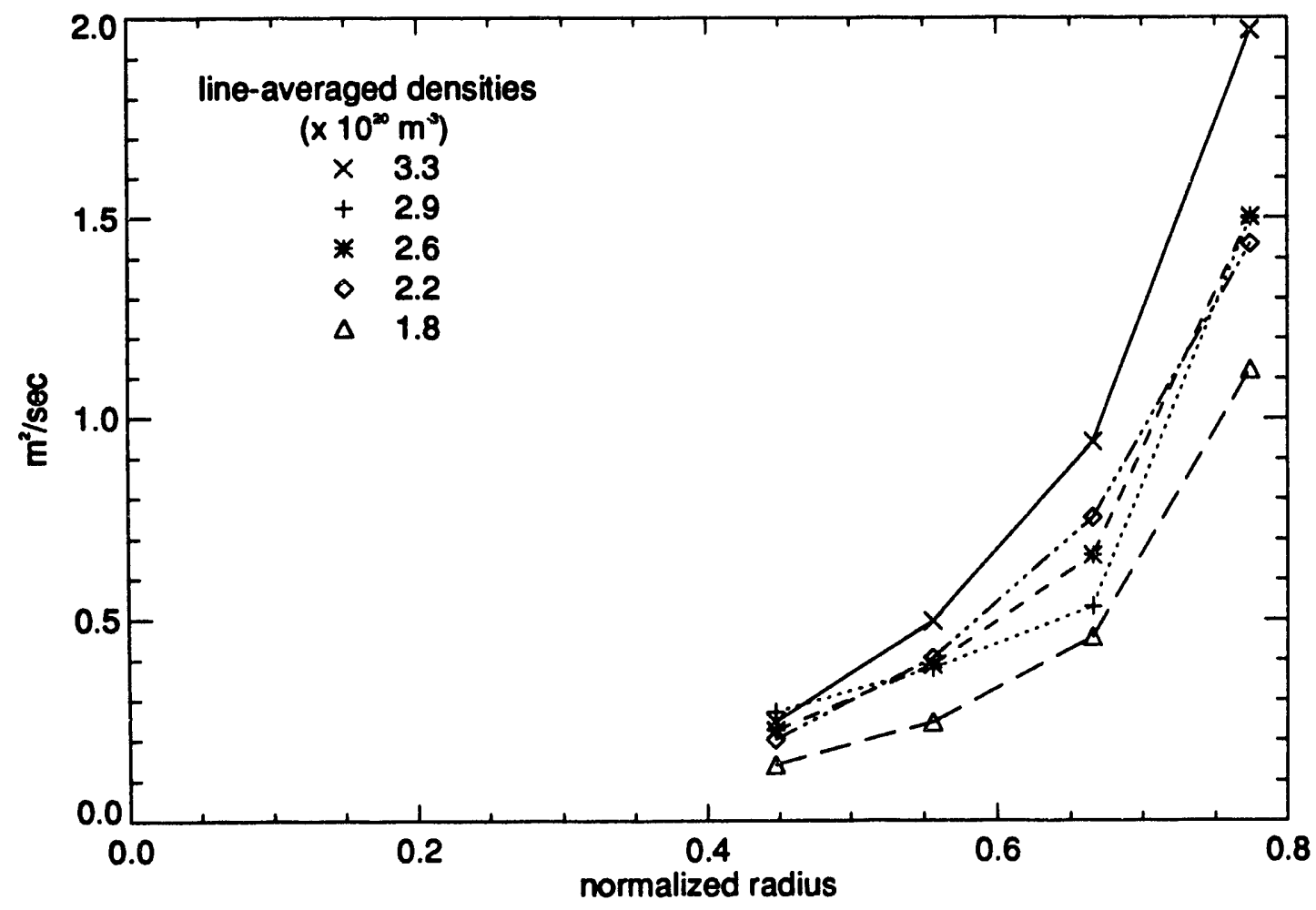

Figure 5.4: Perturbed resistive ballooning coefficients for plasmas of the $285 \mathrm{kA}$ density scan.

Value of $\mathrm{D}_{R B}$ calculated for the profiles of the $285 \mathrm{kA}$ density scan are shown in fig. 5.4. There is some similarity between the scaling of these coefficients with those of the RGDT coefficients for this data. This is because of the similarities in the scalings with $\mathrm{q}, \mathrm{T}$ and $\mathrm{n}$ in the two expressions above and because the electron temperature has a larger fractional variation than other variables in this region of the plasma across the density scan. The increase in $\mathrm{D}_{R B}$ as $\bar{n}_{e}$ is raised is not correlated with a change in the local $L_{n}$ in the data. This was checked by averaging profiles obtained from each of the shots associated with each data point. The dependences in $D_{R B}$ do not appear to be strong enough to match the measured variation in $\mathrm{D}$ across the density scan. The inverse scaling with temperature is the one factor most responsible for causing a pronounced rise in the calculated $\mathrm{D}$ at the density limit. 


\subsection{Other Turbulence Theories}

The model of the radiative-rippling instability driven turbulence examined previously has been generalized and extended to describe the coupled dynamics of radiative instability and drift waves [105]. In this drift-thermal instability-induced turbulence theory purely thermal drive is supported by collisional drift-wave like fluid dynamics. Potential and temperature fluctuation levels and radial correlation lengths have been calculated.

In another recent edge turbulence theory drift waves are driven by ionization causing a purely inward particle flux [106]. This is proposed as an explanation for the rapid particle intake observed during neutral gas puffing. Its authors note that another mechanism would be needed to explain the equilibrium convected flux. The expression given for the inward particle flux is

$$
\Gamma_{n}=-n_{0} c_{s}\left[\frac{\chi_{\|} \bar{k}_{\|}^{2} L_{T}}{\left(\bar{k}_{y} \rho_{s}\right)^{4} c_{s}}\right]\left(\frac{\gamma_{I}^{2}}{\Omega_{i}^{2}}\right)
$$

with $\gamma_{I} \equiv n_{n}<\sigma v>_{I}$.

In MTX this expression predicts a substantial convective particle flux only at the very edge of the plasma. This is because the neutral particle density, and thus $\gamma_{I}$, falls very rapidly as one moves into the plasma. As such this theory cannot explain the anomalous inward perturbed convection velocities measured in the bulk plasma region $(\mathrm{r} / \mathrm{a} \leq 0.8)$ in these gas modulation experiments.

A theory of ion-temperature-gradient-driven turbulence [107] was also considered to explain the anomalous inward convection in the core of the plasma. The expression given for the inward convection velocity was evaluated using profile data and a value of $\eta_{i}$ obtained from the ion temperature profile calculated with ONETWO. For this region of these plasmas the values of $\eta_{i}$ obtained were greater than the theoretical value for threshold of the instability $\eta_{I, c r} \sim 1.5$. The calculated convection velocity was in general too small and did not have a profile with a radial dependence which resembled the measured ones. 


\subsection{Discussion}

It is reasonable to associate particle transport in the peripheral region of the plasma with electrostatic turbulence. Measurements on TEXT [25] show that electrostatic turbulence extends to the edge continuously wiere it becomes the established cause of particle transport. Since the edge has steep density gradients and is dominated by diffusion it is logical to associate this turbulence with interior diffusion [25]. While an explanation of the convective and diffusive components of the flux in terms of separate mechanisms is acceptable, an explanation in terms of a single theory is more plausible. This is underscored by the observation that both the perturbed convection and diffusion coefficients rose together near the density limit while relatively little change occurred in the local shape of the density profile. In the $285 \mathrm{kA}$ density scan the values of $\mathrm{D} / \mathrm{VL}_{n}$ at $\mathrm{r} / \mathrm{a}=0.8$ fall in a similar range independent of density. None of the specific models considered here account for this observation. Indeed no theory explains all of the observed characteristics of turbulence.

The resistive, collisional outer regions of the high-density Alcator $\mathrm{C}$ plasmas considered satisfy conditions for the reduced Braginskii equations to be valid. Although several approximations of the RGDT theory examined are essentially valid in the plasma considered, certain ones, such as assumptions in the derivation of the impurity concentration evolution equation and the neglect of toroidal curvature, encounter difficulties. Without a more direct method of examining the relation between current density and turbulence in the outer region, such as a measure of fluctuations during a current ramp, one cannot establish whether the deterioration in particle confinement near the density limit in Alcator $\mathrm{C}$ is caused by a current-driven mode such as RGDT.

Whatever the mechanism responsible for the enhanced particle transport, the most significant result here is the similarity in the trends of measured particle diffusion coefficients in fig. 4.11 and the scaling given for $\mathrm{D}_{R G D T}$ evaluated for parameters of the relatively cool, collisional outer plasma regions in fig. 5.1 as the density limit is approached. The inverse dependence of the theoretical scaling for D on temperature and/or temperature gradient scale length, characteristic for example of the RGDT or 
resistive ballooning theories, causes it to increase as the temperature profile narrows.

In these gas modulation experiments the global energy confinement time did not deteriorate near the density limit. Also, in the Alcator $\mathrm{C}$ pellet injection experiments no deterioration in energy confinement was observed other than that associated with the convected flux of particles. These observations demonstrate that the anomalous $\chi_{i, e q}$ profile did not increase in a manner similar to the $\mathrm{D}$ profile. The underlying mechanism behaves differently from that responsible for the increase in ion thermal conductivity in the saturated ohmic confinement regime.

We can infer that the same mechanism responsible for the increase in particle transport coefficients is capable of causing an increase in the net particle flux. Global energy confinement in Alcator $\mathrm{C}$ would be weakly influenced by a mechanism which increases only the particle convection. When acting in a region where particle convection accounts for a large fraction of the total thermal flux, such as might occur near the plasma periphery, such a mechanism would cause a significant local increase in thermal transport as well. 


\section{Chapter 6}

\section{Comparison with Behavior on other Tokamaks}

Density limit disruptions are generally thought to be the result of edge plasma cooling, resulting in a steepening of the current density profile about the $q=2$ surface. Radiation, deteriorating particle confinement or scrape-off layer collapse are all mechanisms for edge cooling which appear to play roles in density limit dynamics on the various machines. A deterioration in particle confinement plays an important role in the density limit on several tokamaks, including Alcator C, T-10, Tuman-3, DITE and TEXT. In this chapter we corsider the similarities between the characteristics of the deterioration in particle confinement on the various machines to develop a more unified picture of the underlying mechanism. These characteristics will also be contrasted with those of a plasma which does not exhibit the deterioration in confinement near the density limit. The significance of the scrape-off layer behavior will be discussed. 


\subsection{Location of Region of Confinement Deterio- ration in Plasma Profiles}

Evidence from all of the devices where the deterioration in confinement is observed near the density limit suggests it is caused by a mechanism which acts in regions of relatively low temperature. In the DITE tokamak the outer regions of the plasma show a degradation of particle confinement time as the density limit is approached [126]. A reduction in energy confinement is also observed in the edge plasma as was described in chapter I. As mentioned in that section there was a gradual narrowing of the plasma temperature and current profiles as the density was raised toward the limit.

As noted in chapter I particle transport in the plasma edge also plays a dominant role in the density limit behavior of the TUMAN-3 tokamak [21]. In experiments the density was increased with gas puffing while the current was ramped up concurrently with magnetic compression of the minor radius of the plasma. The plasma density increased in a "steplike" fashion. Periods where the slope of the density increase was reduced coincided with pronounced increases in the $\mathrm{H}_{\alpha}$ signal, implying steplike changes in the global particle confinement time.

In TEXT a transient enhancement of density fluctuation levels associated with microturbulence is observed to precede density limit disruptions [40]. Using measurements of the propagation velocity of the sawtooth heat pulse in the region $0.5 \leq \mathrm{r} / \mathrm{a}$ $\leq 0.8$, and assuming a simple diffusive transport equation, the "effective" values of the perturbed $\chi_{e}$ and $D_{e}$ were calculated. It must be noted that this analysis ignores effects such as the coupled density-temperature response. The density profile evolution considered is not primarily due to the slow wave so that the perturbed D is not necessarily related to the equilibrium $\mathrm{D}$ in the manner described in sec. 2.2 . Off diagonal terms can be manifest in the $\mathrm{D}$ determined by this method. For disrupting shots the value of $\mathrm{D}_{e}$ and $\chi_{e}$ calculated rise continuously and dramatically starting at $\sim 5 \tau_{e}$ before the disruption. On shots where no disruption occurs, no rise in $\mathrm{D}_{e}$ or $\chi_{e}$ is recorded. The time history of the density fluctuation level is measured for a range of wave numbers with FIR laser scattering. For the wavenumber shown and 
parameters given for these shots the range of specified $0.3 \leq k_{\theta} \rho_{s} \leq 0.9$ corresponds to a range of electron temperatures $50 \mathrm{ev}<T_{e}<420 \mathrm{ev}$. The density fluctuation amplitude $s_{k} \sim \tilde{n}_{k}^{2}$ is observed to increase substantially prior to a disruption in a manner similar to the "effective" values calculated for $\chi_{e}, D_{e}$.

Note that the region where time to peak measurements were made and the range of temperatures where the density fluctuations were measured both correspond roughly with those where transport coefficients were enlarged near the density limit in MTX. Measurements of turbulent fluctuations in TEXT with Langmuir probes at the plasma edge and in the scrape-off layer showed no enhancement until the plasma became MHD unstable. A feature in the turbulence spectrum rotating in the ion diamagnetic drift direction measured with FIR scattering grew as the turbulence level increased prior to disruption. This feature is observed only in high-density plasmas when the energy confinement has saturated. Even though the calculated value of $\mathrm{D}_{e}$ increased during the precursor no change in the density profile was apparent relative to that on a non-disrupting shot until the plasma became MHD unstable. Recall that the density profiles in MTX showed no substantial change in shape as the perturbed transport coefficients rose near the density limit. This could be understood because of the increase in the inward convection velocity that accompanied the increase in $\mathrm{D}$. Previous results in TEXTOR have also shown a significant increase in the density fluctuation level as long-time precursors to disruptions [108]. Changes in impurity confinement have been observed near the density limit $[123,48]$.

It is not clear whether the TEXT plasma parameters were evolving significantly during the precursor. The apparent rise in $\mathrm{D}_{e}$ in TEXT is qualitatively similar to that measured on MTX. The increase during the precursor terminates in a disruption on TEXT, contrary to the stable behavior exhibited by the quasi-steady-state density limit plasmas in the gas modulation shots in MTX. Part of the reason for this difference may be because $\mathrm{q}_{a} \sim 3$ for the shots studied in TEXT, quite a bit lower than the $\mathrm{q}_{a}=4.8$ for the MTX shots. Recall that the higher $\mathrm{q}_{a}$ was chosen to avoid disruptions. Again the gas modulation shots studied in MTX were created with quasi-steady-state background profiles and exhibited no significant MHD instabilities. Thus there is no 
apparent evidence that would indicate the increase in particle transport coefficients in MTX near the density limit is a transient event.

\subsection{Relation to Edge Turbulence}

Since the deterioration in confinement occurs in the plasma periphery or the outer portions of the bulk plasma in these machines it is reasonable to examine this behavior in relation to that of turbulence in the low temperature edge regions of the plasma. In separate sets of experiments on TEXT the effect of injected carbon and nitrogen impurities on turbulence in the plasma periphery was examined [43]. These were motivated by the RGDT model with radiative instability of Thayer et al. [92]. With nitrogen puffing, an increase in the floating potential fluctuations and in the particle flux induced by electrostatic fluctuations was observed, while density fluctuations remained essentially unchanged. No change in either the fluctuation levels or the particle flux was observed in the edge plasma when carbon impurities in the form of methane were introduced. For fluctuations on closed flux surfaces the theory predicts the enhancement in the turbulent quantities due to impurity radiation are

$$
\begin{aligned}
\frac{\tilde{n} / n}{(\tilde{n} / n)_{I_{Z}=0}} & \propto\left[\frac{\tilde{\varphi} / T_{e}}{\left(\tilde{\varphi} / T_{e}\right)_{I_{Z}=0}}\right]^{1 / 3} \\
\frac{D}{D_{I_{Z}=0}} & \propto\left[\frac{\tilde{\varphi} / T_{e}}{\left(\tilde{\varphi} / T_{e}\right)_{I_{Z}=0}}\right]^{4 / 3} .
\end{aligned}
$$

In the comparison with the theoretical prediction they used the assumption that the floating potential fluctuations measure approximately plasma potential fluctuations, which is valid only in the limit if temperature fluctuations are much smaller than the floating potential. They also assumed that the cooling rate $\mathrm{I}_{z}$ could be obtained from a shifted coronal equilibrium model. With these assumptions good quantitative agreement was found between the measured density fluctuation levels and those predicted from the theory due to the thermal radiation instability for both the carbon and nitrogen instabilities. According to the model the differences between the effects of the two impurities was due to the differences in their curves of $I_{z}\left(T_{e}\right)$. 
In another set of experiments on TEXT the effect of neon injection on edge and bulk piasma turbulence was examined [4]. In these experiments fluctuations were measured by fir scattering for $\mathrm{r} / \mathrm{a} \sim 0.5-0.85$ and by Langmuir probes for $\mathrm{r} / \mathrm{a} \sim 0.95-$ 1.15. When the cooling rate $I_{z}$ is calculated from a coronal equilibrium model for TEXT temperature profiles the thermal drive for neon is predicted to be most pronounced in the region $\mathrm{r} / \mathrm{a} \sim 1$ ).9. Turbulence was studied in shots with and without neon injection while the density was decreased. Following impurity injection the peripheral temperature decreased, whereas without impurity injection it increased as density was lowered. No changes in the peripheral turbulence $(\mathrm{r} / \mathrm{a} \sim 0.95-1.15)$ were measured by the Langmuir probes following impurity injection. Density fluctuations in the bulk plasma measured by fir scattering showed an increase following impurity injection. When neon was injected into a plasma with a steady state density a dramatic decrease in the electron temperature at the plasma periphery was caused by radiation cooling. An increase in fluctuation levels was measured by the fir in the bulk plasma $\sim 40 \mathrm{msec}$ after injection. Measurements of fluctuations at the plasma periphery were not available during this shot as the Langmuir probes were operating in a different mode. These results were taken as qualitative evidence supporting the radiation drive mechanism described in the rippling/radiative model [92].

Probe measurements from the TJ-1 tokamak discussed in sec. 5.2 .5 also support the notion that radiation is an important driver of edge turbulence [104]. Moreover the radiation cooling of the plasma periphery on TEXT was associated with an increase in the level of tuizbulence in the bulk plasma [4]. Thus the large amounts of radiation emission characteristic of many edge plasmas near the density limit may help to enhance turbulence. The resulting increase in transport would help cool the edge plasma further.

\subsubsection{Relation to Improved Confinement Modes}

The same edge plasma conditions which lead to a density limit appear to preclude attainment of improved confinement modes. At the onset of the high density mode and ohmic $\mathrm{H}$ mede in Tuman-3, characterized by increased density and global energy 
confinement time, there is a substantial drop in the $\mathrm{H}_{\alpha}$ signal and in the measured electrostatic particle flux in the plasma edge [21]. In the high density mode the density limit is increased. These modes are only obtained when the instability responsible for increased edge recycling is suppressed [21]. It is worth noting that an $\mathrm{H}$-modelike regime on $\mathrm{T}-10$, characterized by improved energy confinement, resulted from suppression of edge turbulence which was triggered by changes in the plasma edge.

A study of L-mode, elming $\mathrm{H}$-mode and ohmic discharges with $\mathrm{Z}_{\text {eff }} \leq 2$ near the density limit in DIII-D revealed that low-q plasmas disrupt following the growth of a locked mode, while high-q discharges become detached and remain stable in the absence of locked mode [109]. In the high-q plasmas disruptions are infrequent for both DIII-D divertor and limiter discharges. The density limit in DIII-D is controlled by processes which occur at the plasma edge. According to reference [109]:

"Evidence from a number of discharges is consistent with a picture of the density limit proceeding from outside to the inside of the plasma. Locking of the $2 / 1$ mode was observed before any effect was seen on the central SXR emission. In rnany cases, both the level of the central emission and sawtoothing activity continued after the $2 / 1$ mode had locked."

DIII-D H-mode plasmas always revert to L-mode prior to a density limit disruption. The features of the evolution of a typical elming $\mathrm{H}$-mode density limit shot were discussed. During the H-mode to L-mode transition the edge pressure $(r / a=.9)$ decreases before other portions of the plasma interior change. Before $\mathrm{D}_{2}$ gas was puffed the electron temperature measured at the separatrix was $\sim 150 \mathrm{ev}$, but dropped to $\sim 60 \mathrm{ev}$ after puffing began. This was followed by an increase in the density outside the separatrix. A huge increase in recycling activity was observed along the outboard leg of the divertor. This trend continued as the H-mode edge density pedestal disappeared. At $3150 \mathrm{msec}$, though still in the $\mathrm{H}$-mode the profile had acquired characteristics associated with high-density L-mode such as a relatively cool region near the edge of the plasma and a $T_{e}$ gradient that is rather flat near $r / a=1$. At $3250 \mathrm{~ms} \in c$ the discharge had fallen into L-mode. The temperature at the separatrix was $\sim 45 \mathrm{ev}$ and a region with a low $T_{e}$ gradient extended even further inward. 
Attainment of $\mathrm{H}$-mode is commonly associated with suppression of edge turbulence, perhaps by velocity shear in the edge plasma [112]. The formation of a density pedestal near the plasma periphery, just inside of a region with a very high density gradient, is characteristic. In these DIII-D density limit shots the H- to L-mode transition occurs at densities at or slightly lower than the L-mode density limit. This might be taken as evidence that an increase in edge turbulence plays a role in the density limit in DIII-D. Such turbulence, localized to the edge plasma, might help cause edge cooling in addition to that caused by the radiation.

\subsection{Density Limit Behavior in TFTR}

In a fairly recent set of experiments on TFTR sequential injection of multiple deuterium pellets combined with high power neutral beam heating has produced and sustained plasmas with densities exceeding the Greenwald limit by up to a factor of 2.2 [127]. These experiments were conducted about one week after an amorphous boron-carbon layer had been deposited on the first wall, which resulted in a suppression of metallic and oxygen impurities by roughly a factor of two.

In the most successful shot, six pellets were injected at $250 \mathrm{msec}$ intervals into a plasma with $\mathrm{R}_{p}=2.37 \mathrm{~m}, \mathrm{a}=0.72 \mathrm{~m}, \mathrm{~B}_{T}=5.0 \mathrm{~T}, \mathrm{I}_{p}=1.8 \mathrm{MA}$ and $\mathrm{q}_{a}=3.3$. Neutral beam sources were added $10 \mathrm{msec}$ after each pellet, producing a "staircase" power waveform, in an attempt to provide sufficient power to reheat the plasma before the next pellet. In these experiments, no evidence was found for a dramatic deterioration of the central particle confinement in plasmas fuelled by pellets above the Greenwald scaling limit [127]. The confinement time calculated by TRANSP for particles within the inner half in minor radius actually increased as the density rose with each succeeding pellet. The highest density points produced by pellet fuelling in these experiments do not represent an intrinsic density limit, they are simply the highest points achieved.

It is interesting to compare this plasma with the MTX plasmas considered earlier which exhibited a large increase in particle transport coefficients in the bulk plasma near the Greenwald density limit. Both are circular limiter plasmas with similar 
central densities, $\left(\mathrm{n}_{e, 0}\right.$ near $\left.5 \times 10^{20} \mathrm{~m}^{-3}\right)$ as well as similar toroidal field strengths $(5 \mathrm{~T}$ for TFTR vs. $6.25 \mathrm{~T}$ for MTX). Though there are of course many differences between these plasmas such as the size, current densities and more subtle differences in profile shapes, the most obvious difference between them is in their electron temperature profiles. The TFTR $T_{e}$ profiles are hotter than for these MTX discharges, particularly at the limiter. We saw that in MTX the increase in the transport coefficients which occurred near the density limit was in the collisional, relatively cool outer region of the plasma where $\mathrm{T}_{e}<250 \mathrm{eV}$. The temperature at the limiter for these high-density gas fuelled TFTR shots is $\sim 200 \mathrm{eV}$. Thus the increase in transport coefficients in MTX occurred in a region cooler than most of the TFTR plasma. For the low-current, high-density ohmic MTX shots, this region where $\mathrm{T}_{e}<250 \mathrm{eV}$ extended more than a third of the way into the plasma. But for TFTR such low temperatures are found in the bulk plasma only very near the limiter.

Thus if the increase in particle transport coefficients in Alcator $\mathrm{C}$ is due to an instability which occurs at low $T_{e}$, such as a resistive instability, the resulting deterioration in particle confinement can still occur in a region extending well into the plasma core. In TFTR such an instability could only exist in the very edge region of the plasma. Thus no deterioration in the core particle confinement would occur. If the density limit was influenced by such turbulence it would be manifest as an edge density limit.

In the shots fuelled with pellets and neutral beams shown in ref. [127] the electron temperature at the inner limiter is $\sim 400-500 \mathrm{eV}$. If edge turbulence which is stabilized at elevated temperatures helped to cause edge cooling leading to a disruption, then such an increase in edge $T_{e}$ would help to extend the density limit. Higher edge temperature could, however, also suppress line radiation from that region. Certainly the reduction in impurities due to boronization served to help reduce radiation losses, which are important in the TFTR density limit. .

Particle transport coefficients were determined with the gas modulation technique utilizing a single puff on TFTR [24]. Profiles of transport coefficient were determined for four shots at the same current having very similar density profiles, but with 
different neutral beam injection powers and thus different temperature profiles. The scaling of the particle transport coefficients with electron temperature averaged over the four shots was approximately $\propto \mathrm{T}_{e}^{2}$ over the radial range of the measurement $0.4 \leq \mathrm{r} / \mathrm{a} \leq 0.7$. The scaling with temperature of the "composite" thermal diffusivity defined as

$$
\chi_{\text {flux }}=-\left(Q_{e}+Q_{i}\right) /\left(n_{i} \nabla T_{i}+n_{e} \nabla T_{e}\right)
$$

was found to be virtually identical to that of the particle transport coefficients. A simulation of the plasma was performed involving a kinetic microinstability calculation in toroidal geometry for trapped-particle modes driven by trapped-electron and $\eta_{i}$ dynamics [128]. For $r / a<0.65$ this simulation indicated that the $\eta_{i}$ mode should be dominant in the region. The temperature exponents of the transport coefficients were calculated in the simulation using the mixing length approximation, and was in reasonable agreement with the scaling of the experimental values. The electron temperature in the region $0.4 \leq \mathrm{r} / \mathrm{a} \leq 0.7$ of these TFTR shots varied between $\sim 1-3$ $\mathrm{keV}$ and the density $(2-3.5) \times 10^{19} \mathrm{~m}^{-3}$. The plasma is obviously quite different from the collisional, much cooler, denser plasma in the outer region of the MTX density limit shots.

The $T_{e}$ scalings of the electron and ion thermal diffusivities from the simulation calculation were both the same as for the mixing length assumption. Thus according to the simulation with $\eta_{i}$ dynamics the thermal transport coefficients should scale with electron temperature in the same manner as the particle transport coefficients, as was observed in the experiment on TFTR. This relation between the temperature scalings of particle and thermal transport coefficients is the most important point that contradicts an explanation of the density limit behavior in Alcator $\mathrm{C}$ in terms of the $\eta_{i}$ mode. 


\subsection{Relation of Transport Coefficients to Plasma Profiles on T-10}

The T-10 tokamak shows a pronounced deterioration in particle and energy confinement in the edge plasma as the density limit is approached. As described in chapter I the evolution of the limit shots with ECH has been broken into four stages [33]. In the first stage, as $\bar{n}$ increases during gas puffing, there is a degradation in confinement in at least the plasma exterior and an enhancement in the plasma-wall interaction. The intensity of the $\mathrm{D}_{\alpha}$ line rises more rapidly than $\bar{n}_{e}$ and is mainly associated with emission from the region near the inner torus wall where Marfes exits. The edge electron temperature decreases during this same time period and a cooling front moves inward. During the second stage the $\mathrm{D}_{\alpha}$ signal and density at the plasma periphery are reduced. It is speculated in ref [33] that this may be due to increased shear, resulting from redistribution of the edge plasma current density, acting to stabilize the edge turbulence. The third stage is characterized by well-developed MHD activity leading to the fourth stage during which a disruption occurs.

Dnestrovskij and Yushmanov $[129,130]$ modelled transport in the periphery of the $\mathrm{T}$-10 plasma which they characterized with the conditions $\mathrm{q} \geq 2, \mathrm{~T} \leq 200 \mathrm{ev}, \nu^{*} \geq 1$. The approach they utilized was a simulation of the dynamic evolution of several discharges using local transport coefficients which depended upon local plasma parameters. Scaling of three "most probable" candidate theories of edge turbulence were considered.

These were the collisional part of the Ohkawa type coefficient [131]

$$
\chi^{d s t}=\frac{c^{2}}{\omega_{p e}^{2}} \nu_{e}
$$

the dissipative circulating electron mode [132]

$$
\chi^{d c e}=\frac{\omega_{*}^{2} \nu_{e}}{k^{2} \omega_{t e}^{2}}
$$

and the familiar rippling mode scaling [90]. In the simulation only the dependences upon the temperature, density and $q$ were considered so that the scalings were reduced 
to $\chi^{\text {dst }} \sim 0.1 / T_{e}^{3 / 2}, \chi^{d c e} \sim n q^{2} / T^{1 / 2}$ and $\chi^{r} \sim 10^{-2} q^{2} n^{1 / 3} / T^{5 / 6}$. The particle flux was taken as

$$
\Gamma=-D_{p} \nabla n-V_{p} n
$$

with $\mathrm{D}_{p}=\chi_{e} / 2$ and $\mathrm{V}_{p}=c \frac{E_{\|} r^{2}}{B_{p} a^{2}}\left(1-r^{2} / a^{2}\right) C^{v p}$. The thermal fluxes were taken as purely diffusive. The electron thermal diffusivity in the bulk plasma was taken to be a combination of the peripheral $\chi_{e}$ and the Parail-Yushmanov stochastic coefficient [133], with the latter given by

$$
\chi^{s t}=C^{s t} \epsilon \frac{c^{2}}{\omega_{p e}^{2}} \frac{V_{e}}{q R} \sim \frac{T^{1 / 2}}{n} .
$$

It was stated that the latter scaling was a good approximation for electron thermal transport in the core plasma of T-10. These coefficients are summed so that each makes a dominant contribution in its respective region. In order to avoid discontinuity at the scrape-off layer boundary the sum is limited by Bohm diffusion as

$$
\chi_{e}=\left(\frac{1}{\chi^{s t}+\chi^{e d g e}}+\frac{1}{D_{B o h m}}\right)^{-1}
$$

where $\mathrm{D}_{B o h m}=C^{b m} \frac{1}{16} \frac{c T}{e B}$. The $\mathrm{C}^{b m}, \mathrm{C}^{s t}$ and $\mathrm{C}^{v p}$ are fitting constants.

The scrape-off layer is also modelled and constrained to evolve consistently with the bulk plasma. This 0-dimensional scrape-off layer model includes the effects of ionization of neutrals, radiation energy losses and both longitudinal heat conduction and plasma convection along field lines and anomalous perpendicular Bohm diffusion and heat conductivity.

Simulation of shots with auxilliary heating and gas puffing revealed that both the rippling and dissipative circulating electron models were appropriate to describe approximately the dynamical evolution of the edge plasma [130]. The collisional part of the Ohkawa-type coefficient had a stronger $\mathrm{T}$ dependence which caused the simulation to progress into regimes not observed in the experimental data.

The density limit shots with ECH $\left(q_{a}=3.5-4\right)$ described earlier were simulated in another study using essentially this same model [134]. Transport in the plasma periphery was modelled with the rippling coefficient which was shown by calculation to be the most appropriate description for the $\mathrm{T}-10$ edge region [134]. The association 
of thermal and particle fluxes in the model is reasonable at the plasma edge because of the observation on several machines $[98,99,97,100]$ that the electrostatic particle flux dominates there and can account for a sizable fraction of the total heat flux at the periphery.

The model did a satisfactory job in simulating the dynamics of the evolution of the density limit discharges. The two critical features of the model are the inverse temperature dependence of the edge transport coefficients

$$
\chi^{\top} \sim \frac{n^{1 / 3} q^{2}}{T^{5 / 6}}
$$

and a scrape-off layer density which saturates above a certain level of particle flux. The sharp increase in particle flux and edge losses can be explained by the model only if both of these features are taken into account [135]. This model succeeds in simulating the sharp decrease in the density decay time following pellet injection observed near the density limit in T-10 [39]. The inverse temperature dependence of the transport coefficient can also explain the increase in the limit density with increased auxilliary heating power.

\subsection{Significance of Scrape-Off Layer Behavior}

A scrape-off layer model formulated by Borrass has been advanced as an explanation for the density limit behavior in Asdex discharges [14] and JET discharges with beryllium limiters [136]. The model has several characteristics in common with the treatment of the scrape-off layer given by Dnestrovskij and Yushmanov. Both assume Bohm type perpendicular diffusion in the scrape-off layer. They both give a power scaling of the sol density of $\mathrm{n} \sim P^{5 / 8}$. For ixed heat flow out of the bulk plasma boundary, each model yields an edge density which increases with particle flux through the sol up to a certain level. Above this the sol density essentially saturates, increasing little with the particle flux for fixed power. Both models predict that the sol density should drop as the power flux out of the bulk plasma decreases for fixed particle flux. This mechanism is advanced to explain the drop in the sol density 
following the onset of a Marfe in beryllium limiter density limit discharges in JET. The increase in radiated power from the Marfe decreases the power flux through the sol for fixed total power input into the plasma.

The density in the scrape-off layer in MTX density limit plasmas is also a significant parameter. As we saw in chapter IV DENSEPACK measurements showed the scrape-off layer in Alcator $\mathrm{C}$ is insensitive to the line-averaged plasma density in the regime $\bar{n}_{e} / I_{p}>0.55$ for fixed plasma current. This was consistent with the edge density profiles measured in MTX for the high-density shots of the $285 \mathrm{kA}$ density scan as well as the observation that the scrape-off layer density changed little during gas puffing on these shots. This observation is at least qualitatively consistent with the sol models above which predict a saturation of sol density with fixed input power above a certain level of gas fuelling rate. Since the loop voltage changes only a moderate amount with density in the $285 \mathrm{kA}$ scan and the radiated power fraction is low, constant current is roughly equivalent to constant input power. An increase in particle transport coefficients in the outer bulk plasma is essential to explain results from tokamaks such as Alcator C and T-10. The particle source in gas fuelled Alcator $\mathrm{C}$ plasmas is localized to the scrape-off layer and very edge of the bulk plasma. The rapid increase in particle transport coefficients in the outer plasma near $\bar{n}_{e} / \kappa \bar{J}=1$, combined with the saturation in the scrape-off layer density make the plasma density profile in the region where significant particle source exists insensitive to further increases in this source. This causes the entire density profile to saturate resulting in the density limit in Alcator C.

\subsection{Discussion}

Radiation, deteriorating confinement or scrape-off layer collapse are all mechanisms for edge cooling which appear to play roles in the density limit dynamics on the various machines. Different mechanisms can be more important in different regimes of operation of the same machine. For example, on JET radiation appears to be most significant with carbon limiters, but with beryllium limiters a non-disruptive density 
limit occurs featuring Marfes and increased recycling. The edge density and temperature are key parameters. Deterioration in particle confinement plays an important role in the density limit on several tokamaks, including Alcator C, T-10, Tuman-3, DITE and TEXT. The mechanism responsible for the deterioration in confinement observed on these tokamaks appears to be localized to regions of relatively low temperatures found in the outer region of the bulk plasma. On TEXT an increase in the microturbulence fluctuation level is measured in the outer core plasma and appears to be associated with an increase in transport coefficients. On Alcator $\mathrm{C}$ an increase in the perturbed particle transport coefficients occurs in the collisional, relatively cool outer portion of the plasma. This region of increased transport extends far enough into the core to explain the deterioration of core particle confinement observed in pellet shots near the density limit.

Recent experimental evidence supports theoretical concepts of impurity radiation as a driver of edge turbulence $[43,4,104]$. Radiation cooling of the plasma periphery on TEXT has been been associated with an increase in the level of turbulence in the bulk plasma [4]. Thus the large amounts of radiation emission characteristic of many edge plasmas near the density limit may help to enhance turbulence. The resulting increase transport would help cool the edge plasma further.

Diffusion coefficients obtained in the outer regions of high-density Alcator $\mathrm{C}$ plasmas compare well with the scaling given by a theory of resistivity-gradient-driven turbulence. This demonstrates that no strong dependence upon local plasma parameters is required to explain the dramatic deterioration in particle confinement near the density limit in Alcator C. Global energy confinement data is also consistent with enhanced particle transport due to a type of turbulence which causes little increase in the conducted energy flux.

Deterioration in particle confinement near the density limit is also rather pronounced on T-10. The dynamical evolution of the density limit discharge is also satisfactorily modelled using edge transport coefficients which scale as in the RGDT theory combined with a scrape-off layer model with a density which saturates with respect to particle flux. A scaling from a theory of the dissipative circulating electron 
mode can also be used to model T-10 edge transport. Its dependences on key parameters are similar to those of the resistive ballooning transport coefficient which was compared with MTX data. The overall model succeeds in describing the sharp deterioration in density decay time observed following pellet injection near the density limit in T-10. The inverse temperature dependence of the transport coefficients can also explain the increase in the limit density with increased auxilliary heating power.

These and other experimental observations given suggest that the enhanced transport which has been observed in the outer regions of the bulk plasma on tokamaks such as Alcator C, T-10 and DITE near the density limit is associated with narrowing of the temperature profiles. The coefficient profiles from MTX provide evidence that the mechanism responsible is confined to regions of relatively low temperatures and causes particle transport coefficients to increase through an inverse dependence on $\mathrm{T}_{e}$ and $\mathrm{L}_{T e}$. The T-10, Alcator $\mathrm{C}$ and DITE density limit plasmas studied all have moderately high $\mathrm{q}_{a}$ and have regions of low electron temperature and low electron temperature gradients extending well into the plasma from the limiter. In ohmically heated Alcator $\mathrm{C}$ plasmas the temperature profile narrows with increasing $\mathrm{q}$, and hence decreasing current. Higher radiation losses from the high-density bulk plasma and associated Marfes also cool the edge plasma. An increase in turbulent transport can result in further cooling of the plasma.

In large tokamaks it is observed that the density limit is caused by processes which occur at the plasma edge. If the deterioration in particle confinement is indeed caused by turbulence which occurs only in the lower temperature regions of the plasma, such as a resistive mode, then one would not expect to see any deterioration in particle confinement cause an intrinsic limit to the maximum density in the core of a hotter plasma such as in TFTR. If such a mechanism of turbulence was present in this type of device, the relatively high temperature profile would confine its activity to the plasma periphery. 


\section{Chapter 7}

\section{Conclusions}

The gas modulation technique is a valuable tool for extracting information about particle transport in a plasma. It must be applied with suitable care to ensure that density perturbations create changes in $\mathrm{n}$ and $\nabla \mathrm{n}$ which are small and not correlated, that the plasma remains in a quasi-steady-state during modulation and that the plasma position remains stable. This requires a dedicated experimental effort, particularly for the study of particle transport near the density limit. In MTX both single puff and periodic gas puff modulation could be used at low densities $\left(\bar{n}_{e} \leq 1.6 \times 10^{20} \mathrm{~m}^{-3}\right)$. At higher densities only a single puff modulation could be used because of the increasing density decay time and the limited shot length. Plasma behavior was quite reproducible from shot to shot, facilitating the repetition of the modulation discharge up to 10 times so that statistical errors were minimized.

Perturbed particle diffusion and convection coefficients D, V were obtained. Contributions from off-diagonal transport effects are manifest in V. Coefficient profiles from spectral and time domain analysis of the same periodic modulation are similar. Both the profiles of $\mathrm{V}$ and $\mathrm{D}$ increase toward the outside of the plasma in all of the MTX discharged studied. An anomalous inward pinch exists, and is especially apparent in the outer half of the plasma minor radius. Values of the ratio of $\mathrm{aV} / \mathrm{D}$, shown in fig. 4.13 for $0.4<\mathrm{r} / \mathrm{a}<0.8$, maintain a ratio roughly similar to that expected for the equilibrium coefficients. The dependences of the particle transport coefficients on plasma parameters have a significant influence on values of the perturbed transport 
coefficients.

Profiles of $\mathrm{D}$ are generally similar to those determined previously for $\chi_{e, H P}$ from sawtooth heat pulse propagation [77]. Values of D are typically smaller than those of $\chi_{e, H P}$ by a factor of $2-5$.

The behavior of the transport coefficients was studied for a number of shots at constant current and toroidal field $\left(\mathrm{B}_{T}=6.25 \mathrm{~T}, \mathrm{q}=4.8\right)$ for a range of line-averaged densities extending to the Greenwald density limit. As shown in fig. 4.11, relatively little variation was observed in profiles of $D$ for densities through most of the saturated ohmic confinement regime. At the Greenwald density limit threshold $\left(\bar{n}_{e}=\kappa \bar{J}\right)$ a dramatic increase occurred in both the perturbed particle convection and diffusion transport coefficients in the outer region of the plasma. The increases were most pronounced at the outermost range of the radii where coefficients were determined $(\mathrm{r} / \mathrm{a}=0.8)$. The regions where the increase occurred extended well into the plasma interior.

These results are consistent with the dramatic decrease in the density decay time of the Alcator $\mathrm{C}$ core plasma following pellet injection observed near the density limit. The increase in transport rates is due to the increase in the perturbed diffusion coefficient accompanied by an increase in the perturbed inward convection velocity. Density profiles maintain a similar shape near the density limit, congruous with the similar behavior of the transport coefficients. No dramatic deterioration in global energy confinement is measured.

These increases in the transport coefficients occur in the collisional, relatively low temperature $\left(\mathrm{T}_{e}<250 \mathrm{eV}\right)$ outer portion of the plasma. In these high-density plasmas, these regions were in the Pfirsch-Schlüter collisionality regime. The variation of $D$ in this region compared well with the scaling from a theory of resistivity-gradient-driven turbulence. There is no need to invoke a strong scaling with local plasma parameters to explain the increase in transport coefficients in the data. In the RGDT theory thermal transport is associated almost entirely with convection by the electrostatic particle flux. Such a mechanism for enhanced transport would be consistent with the observed absence of deterioration in the energy confinement time in the data. It 
would also be consistent with the observation from the Alcator $\mathrm{C}$ pellet experiments that the only loss in energy confinement was the convective loss directly associated with the flux of particles.

The T-10 device is another tokamak where the deterioration in particle confinement near the density limit is rather apparent. The dynamics of T-10 density limit discharges were simulated with a model which utilized an RGDT-type scaling for transport coefficients in the plasma edge. The model did a satisfactory job of simulating features of the dynamic evolution of the discharge. The deterioration in edge particle confinement is reproduced primarily because of the inverse temperature dependence of the edge transport coefficients. The inverse dependence on $\mathrm{T}_{e}$ and $\mathrm{L}_{T e}$ is primarily responsible for the increase in D calculated from the RGDT scaling for the MTX density limit data. This observation suggests that the increase in edge transport coefficients is related to the narrowing of the $\mathrm{T}_{e}$ profiles observed at high density through a similar type of inverse dependence.

The MTX data shows that the increase in the particle transport coefficients near the density limit is most pronounced at the largest radii where coefficients were measured. This suggests that the increase may be due to an enhancement of an instability which occurs only in the relatively cool regions of the plasma, such as a resistive instability. In this case the mechanism could not directly cause a deterioration of particle confinement through turbulent transport in the core plasma in a device with substantially hotter temperature profiles. If present in such a device, its action would be confined to the plasma periphery. 


\section{Appendix A}

\section{Calculation of Refraction Angle}

Consider the refraction angle introduced as a beam traverses the plasma. The eikonal approximation is appropriate when the beam wavelength is very small compared to the distance over which the plasma index of refraction changes. The wave fields are represented as

$$
\begin{aligned}
& \mathbf{E}_{\mathbf{0}}=\mathbf{e}_{(\mathbf{r})} e^{i \frac{\omega}{c} S(\mathbf{r})-i \omega t} \\
& \mathbf{H}_{\mathbf{0}}=\mathbf{h}_{(\mathbf{r})} e^{i \frac{w}{c} S(\mathbf{r})-i \omega t}
\end{aligned}
$$

with $S(\mathbf{r})$ defined as the optical path. The surfaces of constant $S(r)$ are the geometrical wave-fronts [65]. Two of Maxwell's equations are fourier transformed in time and expressed in terms of the plasma dielectric constant for the case of $\mathrm{O}$-mode propagation, $\kappa^{2}=1-\frac{\omega_{p e}^{2}}{\omega^{2}}$,

$$
\begin{aligned}
& \nabla \times \mathbf{H}=-i \omega \kappa^{2} \mathbf{E} \\
& \nabla \times \mathbf{E}=i \omega \mathbf{B} .
\end{aligned}
$$

By substituting in the eikonal expressions for the field, and using some vector identities one obtains

$$
\begin{aligned}
\nabla S \times \mathbf{h}+c \epsilon_{0} \kappa^{2} \mathbf{e} & =\frac{c}{i \omega} \nabla \times \mathbf{h} \\
\nabla S \times \mathbf{e}-c \mu_{0} \mathbf{h} & =-\frac{c}{i \omega} \nabla \times \mathbf{e} .
\end{aligned}
$$


In the eikonal approximation the terms on the right hand sides of the above equations are negligible. The fields can be eliminated to obtain the eikonal equation

$$
\left(\frac{\partial S}{\partial x}\right)^{2}+\left(\frac{\partial S}{\partial y}\right)^{2}+\left(\frac{\partial S}{\partial z}\right)^{2}=\kappa^{2} .
$$

which is a special case of the Hamilton-Jacobi equation. Using the transformation

$$
p_{i}=\frac{\partial S}{\partial q_{i}}=\frac{c k_{i}}{\omega}
$$

and defining $\mathrm{q}$ as the spatial coordinate we obtain the Hamiltonian

$$
H(p, q, t)=p^{2}-1+\omega_{p e}^{2} / \omega^{2}=0
$$

from which the $\mathrm{O}$-mode dispersion relation is obtained

$$
\omega^{2}=\omega_{p e}^{2}+c^{2} k^{2} .
$$

The solution to the eikonal equation for a given set of boundary conditions gives the ray orbits which foliate a Lagrangian manifold in phase space. The canonical equations of Hamilton are used to obtain a set of first order relations equations for these orbits

$$
\dot{q}_{i}=\frac{\partial H}{\partial p_{i}}, \quad-\dot{p}_{i}=\frac{\partial H}{\partial q_{i}} .
$$

Since the plasma density is far below the cutoff density for the fir laser wavelength the refraction angle is small and the ray path through the plasma is nearly straight. In this case the beam path through the plasma is approximately along a line parallel to the $\mathrm{y}$ axis a distance $\mathrm{x}$ from the plasma axis. The differential change in the angle of refraction $\approx d k_{x} / k$ as the beam traverses a differential segment dy is

$$
\frac{d k_{x}}{d \tau} / k \frac{d y}{d \tau}=\frac{-\frac{1}{c \omega} \frac{\partial \omega_{p e}^{2}}{\partial x}}{\frac{2 c}{\omega} k_{y} k} \approx-\frac{1}{2 c^{2} k^{2}} \frac{\partial \omega_{p e}^{2}}{\partial x} .
$$

With $\omega_{p e}^{2} / \omega^{2} \equiv n / n_{c}$ and $c^{2} k^{2}=\omega^{2}-\omega_{p e}^{2} \simeq \omega^{2}$ the integral for the refraction angle becomes

$$
\Delta \Theta=-\frac{1}{2 n_{c}} \int_{-\infty}^{\infty} \frac{\partial n}{\partial x} d y .
$$

With use of the relation

$$
\frac{\partial n}{\partial x}=\frac{\partial n}{\partial r} \frac{x}{r}
$$


this integral can be parameterized in terms of the radial coordinate as

$$
\Delta \Theta=-\frac{x}{n_{c}} \int_{R}^{\infty} \frac{\partial n}{\partial r} \frac{d r}{\sqrt{r^{2}-x^{2}}} .
$$




\section{Appendix B}

\section{Spectral Form of Continuity}

\section{Equation}

The terms in the continuity equation with dimensions of length are normalized to the radius of the last closed flux surface yielding

$$
\hat{D}=\frac{D}{\left(a\left(1-\left|\delta_{0}\right|\right)\right)^{2}}, \quad \hat{V}=\frac{V}{a\left(1-\left|\delta_{0}\right|\right)},
$$

which have units of inverse time. The integral form of the continuity equation in normalized geometry becomes

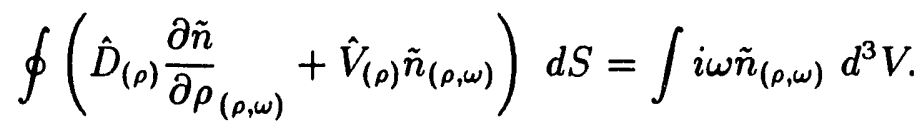

\section{B.1 Finite Difference Equations for Spectral Analysis}

Density profile coefficients are averaged over groups of four adjacent radial positions in the original 50 point grid

$$
n\left(\bar{\rho}_{j}\right)=\frac{1}{4} \sum_{i=1}^{4} n\left(\rho_{4 j+i}\right)
$$

in order to obtain a smoother profile of values at 12 radial positions. This reduced number of flux surfaces is comparable to the number of chord measurements. Second 
order spatial differencing is used to calculate the density gradient

$$
\frac{\partial n}{\partial \rho}=\frac{n_{j+1}-n_{j-1}}{2 \Delta \rho_{j}}, \quad n_{j} \equiv n\left(\rho_{j}\right)
$$

except at the grid boundaries where first order differencing is applied.

The integral form of the continuity equation is discretized as follows

$$
\begin{aligned}
n_{j, \omega} \hat{V}_{j}+ & \frac{\partial n}{\partial \rho_{j, \omega}} \hat{D}_{j}=i \omega n_{1, \omega} \frac{\Delta \rho_{1}}{2}+ \\
& \frac{i \omega}{\rho_{j}} \sum_{i=2}^{j} \frac{\left(n_{i, \omega} \rho_{i}+n_{i-1, \omega} \rho_{i-1}\right)}{2} \Delta \rho_{j} .
\end{aligned}
$$

The first term on the right hand side results from the inner boundary flux surface having a non-zero radius.

\section{B.2 Finite Difference Equations for Simulation of Periodic Modulation}

The differential form of the spectral continuity equation

$$
i \omega n=\frac{1}{\rho} \frac{\partial}{\partial \rho}\left(\rho\left(\hat{D} \frac{\partial n}{\partial \rho}+\hat{V} n\right)\right)+S
$$

is differenced as

$$
\begin{gathered}
\frac{[\hat{D} \rho]_{j+1 / 2}\left(n_{j+1}-n_{j}\right)}{\left(\Delta \rho_{j}\right)^{2}}-\frac{[\hat{D} \rho]_{j-1 / 2}\left(n_{j}-n_{j-1}\right)}{\left(\Delta \rho_{j}\right)^{2}}+\left(\frac{\rho_{j+1} \hat{V}_{j+1}-\rho_{j-1} \hat{V}_{j-1}}{2 \Delta \rho}\right) n_{j}+ \\
\rho_{j} \hat{V}_{j}\left(\frac{n_{j+1}-n_{j-1}}{2 \Delta \rho}\right)=\rho_{j}\left(S_{j}-i \omega n_{j}\right)
\end{gathered}
$$

where

$$
\begin{aligned}
{[\hat{D} \rho]_{j-1 / 2} } & =\frac{1}{2}\left(\hat{D}_{j-1} \rho_{j-1}+\hat{D}_{j} \rho_{j}\right) \\
{[\hat{D} \rho]_{j+1 / 2} } & =\frac{1}{2}\left(\hat{D}_{j+1} \rho_{j+1}+\hat{D}_{j} \rho_{j}\right) \\
S_{j} & =S_{0} \exp \left[-\frac{\xi\left(\rho-\rho_{\max }\right)}{a\left(1-\left|\delta_{0}\right|\right)}\right]
\end{aligned}
$$

This tridiagonal set of equations can be expressed in the form

$$
A_{j} n_{j-1}+B_{j} n_{j}+C_{j} n_{j+1}=S_{j} \rho_{j}
$$


with

$$
\begin{aligned}
A_{j} & =\frac{[\hat{D} \rho]_{j-1 / 2}}{\left(\Delta \rho_{j}\right)^{2}}-\frac{\rho_{j} \hat{V}_{j}}{2 \Delta \rho_{j}} \\
B_{j} & =\frac{\left([\hat{D} \rho]_{j+1 / 2}+[\hat{D} \rho]_{j-1 / 2}\right)}{\left(\Delta \rho_{j}\right)^{2}}+i \omega \rho_{j}+\frac{\left(\rho_{j+1} \hat{V}_{j+1}-\rho_{j-1} \hat{V}_{j-1}\right)}{2 \Delta \rho_{j}} \\
C_{j} & =\frac{[\hat{D} \rho]_{j+1 / 2}}{\left(\Delta \rho_{j}\right)^{2}}+\frac{\rho_{j} \hat{V}_{j}}{2 \Delta \rho_{j}} .
\end{aligned}
$$

The boundary conditions for the simulation are $\left.\frac{\partial n}{\partial \rho}\right|_{0}=0$ and $n\left(\rho_{N}\right)=0$. The inner boundary condition is implemented with second order accuracy. The equations are recast into tridiagonal form by adding a suitable multiple of the second row to the first.

$$
\mathbf{x}=\left[\begin{array}{ccccccc}
-\mathbf{X} \cdot \mathbf{n}=\mathbf{S} \\
\mathbf{X}+3+A_{2} / C_{2} & 8 / 3+B_{2} / C_{2} & 0 & 0 & \ldots & 0 & 0 \\
A_{2} & B_{2} & C_{2} & 0 & \ldots & 0 & 0 \\
0 & A_{3} & B_{3} & C_{3} & \ldots & 0 & 0 \\
\vdots & \vdots & \vdots & \vdots & \ddots & 0 & 0 \\
0 & 0 & 0 & 0 & A_{N-1} & B_{N-1} & C_{N-1} \\
0 & \cdots & 0 & 0 & 0 & 1
\end{array}\right]
$$




\section{Appendix C}

\section{Equations for Analysis in the Time Domain}

Profiles of $n(\rho, t)$ are obtained for each time step from the singular value decomposition solution. The averaged values of density and density gradient are used in the integral form of the normalized continuity equation all according to the conventions adopted in Appendix B. This equation

$$
\int \frac{\partial n}{\partial t} d^{3} V=\oint\left(\hat{D}(\rho) \frac{\partial n}{\partial \rho}(\rho, t)+\hat{V}(\rho) n(\rho, t)\right) d S
$$

is evaluated at discrete points as

$$
\begin{aligned}
n\left(\rho_{j}, t_{k}\right) \hat{V}\left(\rho_{j}\right)+ & \frac{\partial n}{\partial \rho}\left(\rho_{j}, t_{k}\right) \hat{D}\left(\rho_{j}\right)=\frac{\partial n}{\partial t}\left(\rho_{1}, t_{k}\right) \Delta \rho_{1}+ \\
& \frac{1}{2 \rho_{j}} \sum_{i=2}^{j}\left(\frac{\partial n}{\partial t}\left(\rho_{i}, t_{k}\right) \rho_{i}+\frac{\partial n}{\partial t}\left(\rho_{i-1}, t_{k}\right) \rho_{i-1}\right) \Delta \rho_{i} .
\end{aligned}
$$

The time derivative of density is evaluated with a centered differencing formula:

$$
\frac{\partial n}{\partial t}\left(\rho_{j}, t_{k}\right)=\frac{n\left(\rho_{j}, t_{k+\Delta k}\right)-n\left(\rho_{j}, t_{k-\Delta k}\right)}{2 \Delta t \Delta k} .
$$

Linear regression analysis is then applied to determine the transport coefficients. 


\section{C.1 Equations for Simulation of Modulation Data}

The differential form of the normalized continuity equation is differenced in space with the formula

$$
\begin{gathered}
\frac{[\hat{D} \rho]_{j+1 / 2}\left(n_{j+1}-n_{j}\right)}{\left(\Delta \rho_{j}\right)^{2}}-\frac{[\hat{D} \rho]_{j-1 / 2}\left(n_{j}-n_{j-1}\right)}{\left(\Delta \rho_{j}\right)^{2}}+\left(\frac{\rho_{j+1} \hat{V}_{j+1}-\rho_{j-1} \hat{V}_{j-1}}{2 \Delta \rho}\right) n_{j}+ \\
\rho_{j} \hat{V}_{j}\left(\frac{n_{j+1}-n_{j-1}}{2 \Delta \rho}\right)=\rho_{j}\left(S_{j}-\frac{\partial n_{j}}{\partial t}\right) \\
S_{j}=S_{0} \exp \left[-\frac{\xi\left(\rho-\rho_{\max }\right)}{a\left(1-\left|\delta_{0}\right|\right)}\right]
\end{gathered}
$$

The boundary conditions are the same ones used for the simulation in the spectral case of Appendix B. The semi-implicit Crank-Nicholson method is used for time differencing, resulting in the following tridiagonal form for the equations

$$
\begin{aligned}
& A_{j} n_{j-1}^{k-1}+\left(\frac{\rho_{j}}{\Delta t}+B_{j}\right) n_{j}^{k-1}+C_{j} n_{j+1}^{k-1} \\
& \quad=-A_{j} N_{j-1}^{k}+\left(\frac{\rho_{j}}{\Delta t}-B_{j}\right) n_{j}^{k}-C_{j} n_{j+1}^{k}+\frac{1}{2}\left(S_{j, k}+S_{j, k-1}\right)
\end{aligned}
$$

with

$$
\begin{aligned}
A_{j} & =\frac{[\hat{D} \rho]_{j-1 / 2}}{2\left(\Delta \rho_{j}\right)^{2}}-\frac{\rho_{j} \hat{V}_{j}}{4 \Delta \rho_{j}} \\
B_{j} & =\frac{\left([\hat{D} \rho]_{j+1 / 2}+[\hat{D} \rho]_{j-1 / 2}\right)}{2\left(\Delta \rho_{j}\right)^{2}}+\frac{\left(\rho_{j+1} \hat{V}_{j+1}-\rho_{j-1} \hat{V}_{j-1}\right)}{4 \Delta \rho_{j}} \\
C_{j} & =\frac{[\hat{D} \rho]_{j+1 / 2}}{2 \Delta \rho_{j}^{2}}+\frac{\rho_{j} \hat{V}_{j}}{2 \Delta \rho_{j}} .
\end{aligned}
$$

The inner boundary condition is implemented to second order accuracy according to the formula used in Appendix B. The equations are cast in tridiagonal form by adding a suitable multiple of the second row to the first. These equations

$$
\mathbf{L} \cdot \mathbf{n}^{k}=\mathbf{R} \cdot \mathbf{n}^{k-1}+\frac{1}{2}\left(\mathbf{S}^{k}+\mathbf{S}^{k-1}\right)
$$

have the solution

$$
\mathbf{n}^{k}=\mathbf{L}^{-1}\left[\mathbf{R} \cdot \mathbf{n}^{k-1}+\frac{1}{2}\left(\mathbf{S}^{k}+\mathbf{S}^{k-1}\right)\right]
$$


with the associate matrices given by

$$
\begin{aligned}
& \mathbf{L}=\left[\begin{array}{ccccccc}
-\frac{5}{3}+\frac{A_{2}}{C_{2}} & \frac{8}{3}+\frac{\left(B_{2}+\frac{\rho_{2}}{C_{t}}\right)}{C_{2}} & 0 & 0 & \ldots & 0 & 0 \\
A_{2} & \frac{\rho_{2}}{\Delta t}+B_{2} & C_{2} & 0 & \ldots & 0 & 0 \\
0 & A_{3} & \frac{\rho_{2}}{\Delta t}+B_{3} & C_{3} & \ldots & 0 & 0 \\
\vdots & \vdots & \vdots & \vdots & \ddots & 0 & 0 \\
0 & 0 & 0 & 0 & A_{N-1} & \frac{\rho_{N-1}}{\Delta t}+B_{N-1} & C_{N-1} \\
0 & \ldots & 0 & 0 & 0 & 0 & 1
\end{array}\right] \\
& \mathbf{R}=\left[\begin{array}{ccccccc}
-\frac{A_{2}}{C_{2}} & \frac{\left(B_{2}-\rho_{2} / \Delta t\right)}{C_{2}} & 0 & 0 & \cdots & 0 & 0 \\
-A_{2} & \frac{\rho_{2}}{\Delta t}-B_{2} & -C_{2} & 0 & \ldots & 0 & 0 \\
0 & -A_{3} & \frac{\rho_{3}}{\Delta t}-B_{3} & -C_{3} & \ldots & 0 & 0 \\
\vdots & \vdots & \vdots & \vdots & \ddots & 0 & 0 \\
0 & 0 & 0 & 0 & -A_{N-1} & \frac{\rho_{N-1}}{\Delta t}-B_{N-1} & -C_{N-1} \\
0 & \cdots & 0 & 0 & 0 & 0 & 0
\end{array}\right] \\
& \mathbf{S}=\left[\begin{array}{c}
S_{2}^{k-1 / 2} \rho_{2} / C_{2} \\
S_{2}^{k-1 / 2} \rho_{2} \\
S_{3}^{k-1 / 2} \rho_{3} \\
\vdots \\
S_{N-1}^{k-1 / 2} \rho_{N-1} \\
0
\end{array}\right]
\end{aligned}
$$




\section{Appendix D}

\section{Technicalities of Experimental Hardware}

The 15-chord far infrared interferometer used to measure density profiles on MTX is discussed in this appendix along with some details of the gas fuelling system installed for these experiments.

\section{D.1 Interferometer Design}

The interferometer laser is operated at a wavelength of $184 \mu \mathrm{m}$ and detected with corner-cube GaAs Schottky detectors [44]. The laser is located in a room outside of the tokamak vault in order to allow access to it during tokamak operations. The beam is transported to the vault in a large-diameter hollow dielectric waveguide [45] filled with dry air. The detectors and optics are supported by a tower which is independent of the tokamak support structure in order to minimize the effect of vibrations on resolution. Besides floor vibrations, eddy currents from the air-core ohmic heating coil were of concern. To maximize strength, while minimizing eddy current effects, a hybrid stainless steel/G10 design was used [46].

Noise levels measured during plasma breakdown shots with all of the magnets energized. Low frequency components (10's of $\mathrm{Hz}$ ) have an RMS amplitude of $\mathrm{n}_{\ell}=$ $2 \times 10^{17} \mathrm{~m}^{-2}$. The signals are low pass filtered with a cutoff frequency of $4 \mathrm{kHz}$ and 
digitized at $10 \mathrm{kHz}$ with a set of 10-bit LeCroy 8210 digitizers. The interferometer is calibrated in place, using the same filters and digitizers as during plasma operation. A thin polyethylene slab is inserted into the plasma probe beam and rotated using a small motor, changing the effective path length. This allows the digitized signal from the phase comparator to be compared with the phase shift measured on an oscilloscope. The absolute accuracy of the calibration is estimated at $\pm 1 \%$ immediately following the procedure.

\section{D.2 Gas Fuelling System}

In order to facilitate operations for gas modulation experiments, a computer-controlled gas fuelling system was installed on MTX. This was a modified version of the system used on the Tandem Mirror Experiment [27] which could fire two valves independently. Waveforms for the gas valve voltage could be created and modified on the system's Hewlett Packard 9836 computer through a number of options, one of which was simply to draw it. Waveforms could be stored on disk for quick recall. These were loaded into LeCroy 8201 memory modules. The outputs of the associated 8601 function generators were amplified by Kepco bipolar power supplies which drove the PV-10 piezoelectric leak valves. Transducers measured the pressure on the output side of the valves. This was recorded by the gas box system and could be used to assess the performance of the valves. 


\section{Appendix E}

\section{Lowpass / Bandstop Filter}

When selecting a filter one must choose between a set of characteristics that represent the best compromise for the given application. When interferometer signals break up it occurs most frequently in the very last stages of a shot, after the modulation transient is essentially complete. For this reason a causal digital filter was selected for this application. The response of the filter function to fringe shifts late in the shot occurs after the fringe shift and does not contaminate the segment of data during gas modulation. Another selection which must be made is the balance between the dispersion of the filter and the rate of fall off above the cutoff frequency. A Chebyshev filter gives a large amount of attenuation in the stop band for a given amount of "ripple" in the transmission amplitude in the pass band. The rate of change of the filter function phase in the pass band is, however, the most nonlinear [28] and leads to dispersion in the filtered signal. A Maximum Flat Time Delay filter has excellent phase shift characteristics for use when the filter amplitude need not display a rapid attenuation just above cutoff. For the present application Butterworth filters were chosen because they offered a good compromise between flat delay and constant amplitude of the pass band of the function.

A 5 pole Butterworth filter utilized for the lowpass filter function is given by the expression

$$
B_{5}(p)=\frac{1}{1+3.236068 p+5.236068 p^{2}+5.236068 p^{3}+3.236068 p^{4}+p^{5}}
$$


for $0 \leq p<\infty$. The analytic form for $B(p)$ shown in equation $E .1$ is transformed to the form appropriate for the finite frequency domain of the digital record through the following change of variables, using the standard bilinear transformation [28]. For the lowpass filter this becomes

$$
p=C \frac{1-z^{-1}}{1+z^{-1}}=C \tanh \left(\frac{s T}{2}\right)
$$

where $z=\exp s T, s=j \omega$ and $T=t(1)-t(0)$. The coefficient $C=\cot \left(\frac{\pi f_{c}}{2 f_{n}}\right)$ with $\mathrm{f}_{c}$ the filter cutoff frequency and $f_{n}$ the Nyquist frequency. A band stop filter is created with use of the following transformation

$$
p=\frac{D\left(1-z^{-2}\right)}{1-E z^{-1}+z^{-2}}
$$

with $D=\tan \left(\frac{\pi B}{2 f_{n}}\right), \mathrm{B}=$ filter bandwidth, $E=2 \cos \left(\frac{\pi f_{2}}{f_{n}}\right)$ where $\mathrm{f}_{2}=$ bandstop center frequency. The filter function was optimized for this application with addition of two separate two pole Butterworth bandstop filters,

$$
B_{2}(p)=\frac{1}{1+1.4142136 p+p^{2}}
$$

centered at 60 and $110 \mathrm{~Hz}$ cascaded with the lowpass filter. The composite function is thus the product of the three individual functions. The optimum low pass filter cutoff frequency was found to be $90 \mathrm{~Hz}$. 


\section{Appendix F}

\section{Spectral Analysis Methods}

Two methods for determining the particle transport coefficients based upon spectral analysis are given in this appendix. A spectral analysis method was used on data with periodic gas modulation taken at low density.

\section{F.1 Issues Associated with Finite Fourier Trans- form}

Prior to Fourier analysis the average slope of the interferometer signal must be subtracted off. If this is not done the approximation of a linear perturbation about a steady-state background profile is not valid. The time evolution of the background profile would also have to be considered in the analysis. But as long as the changes in the background profile are slow compared with the periodic modulation of the signal the effect of changing background conditions can be minimized by subtracting off the average slope.

Since only a few periods of modulation can be completed during a shot in MTX it is also important that the numerical side-lobe leakage be minimized. This effect is due to the finite time length of the record analyzed. The finite Fourier transform of a signal $\mathrm{x}(\mathrm{t})$ can be represented as the product of an unlimited time history record 


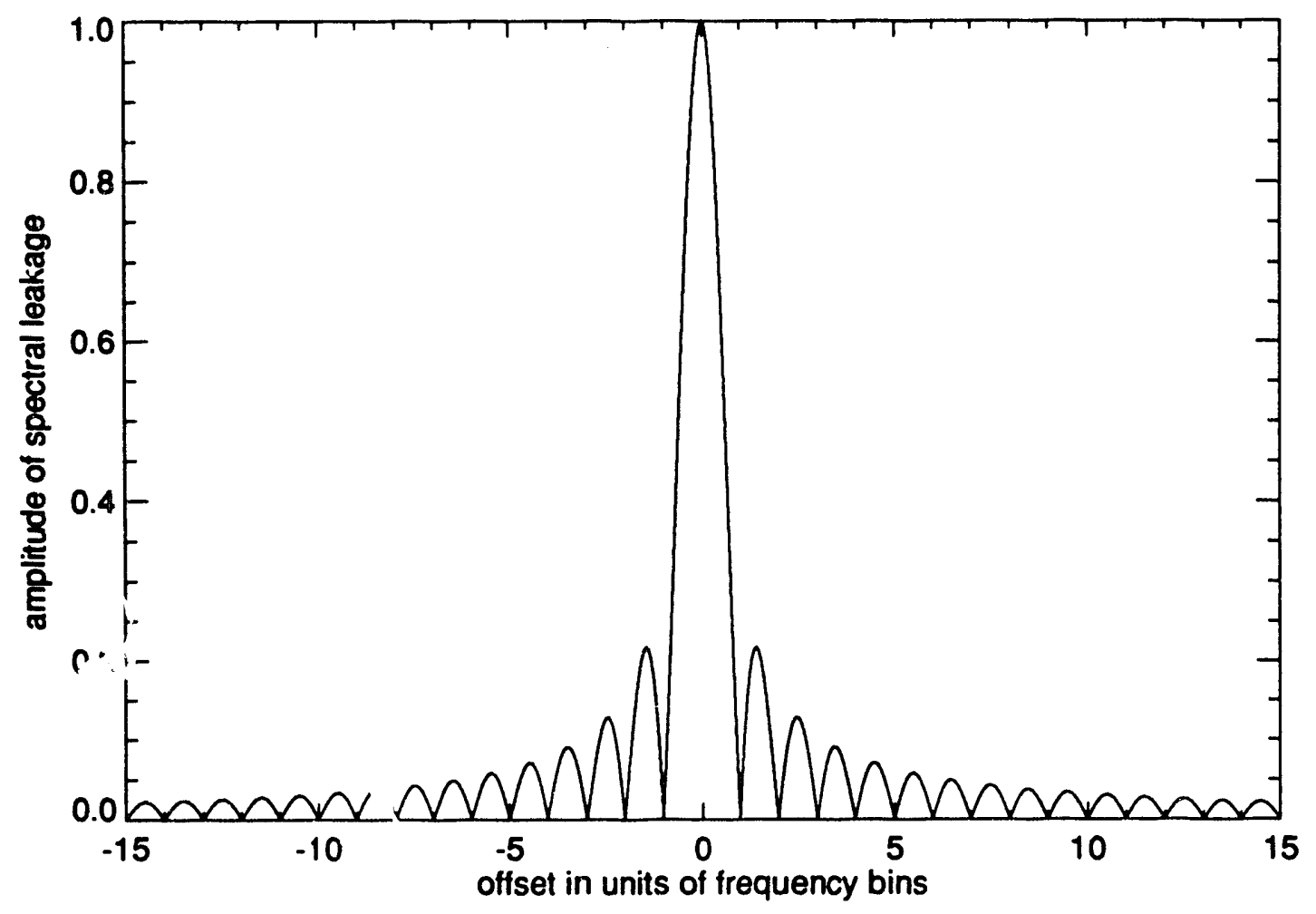

Figure F.1: Plot of $|\mathrm{U}(\mathrm{f})|$ versus frequency

$\mathrm{V}(\mathrm{t})$ multiplied by a rectangular time window $\mathrm{u}(\mathrm{t})$ where [58]

$$
\begin{gathered}
u(t)= \begin{cases}1 & 0 \leq t \leq T \\
0 & \text { otherwise }\end{cases} \\
x(t)=u(t) V(t) .
\end{gathered}
$$

The Fourier transform of $x(t)$ is the convolution of the Fourier transforms $u(t)$ and $V(t):$

$$
X(f)=\int_{-\infty}^{\infty} \mathrm{u}(\beta) \mathrm{V}(f-\beta) d \beta .
$$

The Fourier transform of $\mathrm{u}(\mathrm{t})$ is given by

$$
U(f)=T\left(\frac{\sin \pi f T}{\pi f T}\right) e^{-j \pi f T}
$$

with $T=t(1)-t(0)$ and $f$ the frequency. This is illustrated fig F.1. The large side lobes allow spectral power to leak to frequencies far from the main lobe of the spectral window. This is particularly significant for the analysis here since the fundamental modulation frequency is only a few times the fundamental frequency of the transform; 

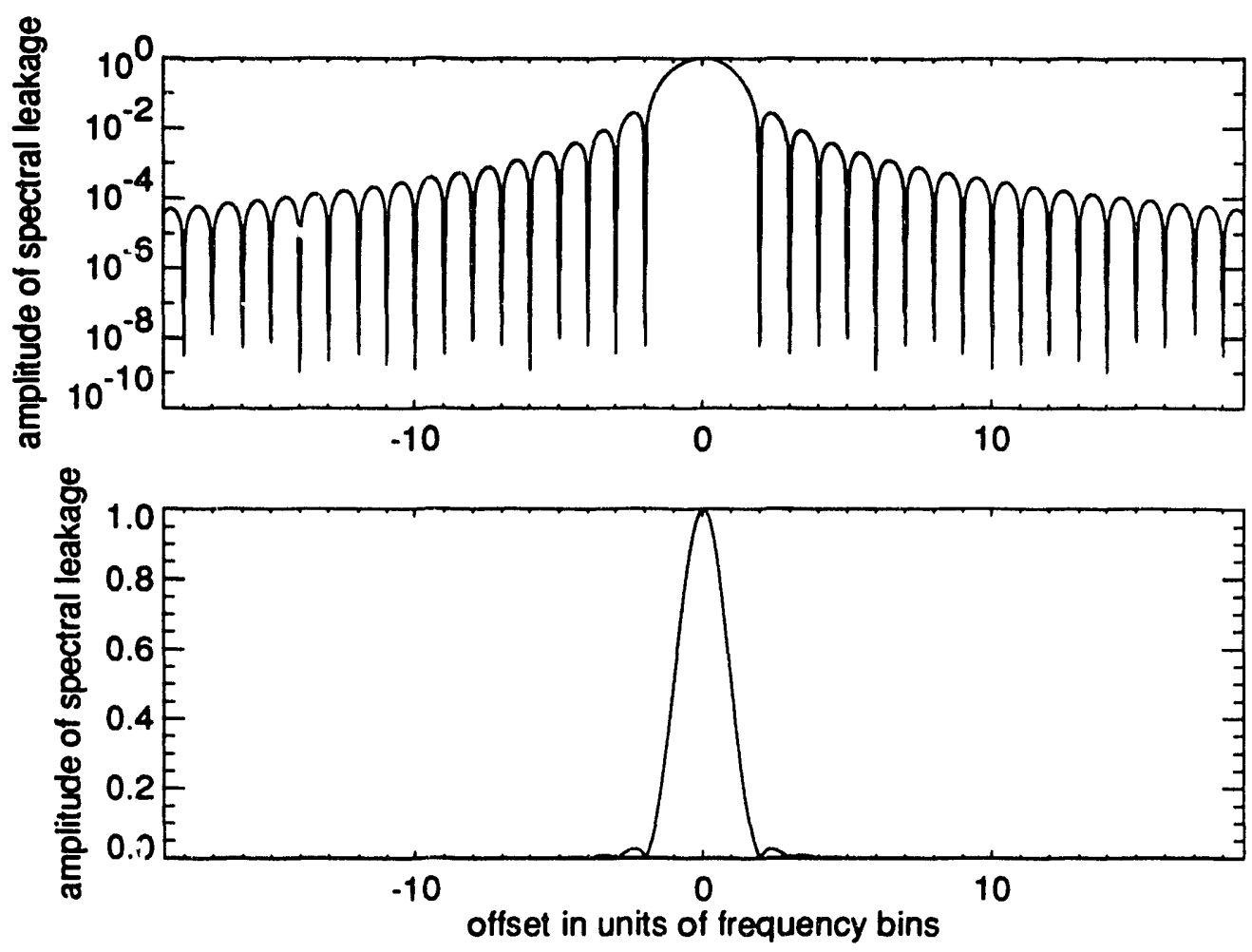

Figure F.2: Fourier transform of Hanning function.

low frequency "noise" of drift in the data can easily leak into the modulation frequency bins, introducing significant distortion. If the record length is selected such that it is exactly an integral number of modulation periods, the modulation component itself will not be subject to leakage, resulting in an improvement in the signal to noise ratio. Leakage of other components in the signal is minimized through use of a window function. This tapers the time history of the signal to eliminate the discontinuities in the signal and its first derivative at its ends. A Hanning window was found to be most effective for this application and is given by

$$
H(t)=\frac{1}{2}\left(1-\cos \frac{2 \pi t}{T}\right) \quad 0 \leq t \leq T
$$

The magnitude of the Fourier transform of $\mathrm{H}(\mathrm{t})$, plotted in figure F.2, shows it has a fairly narrow central lobe and a rapid fall off of the side lobes. 


\section{F.2 Spectral Quantities}

The autocorrelation power spectral density of a signal is

$$
G_{x x}(f)=X^{*}(f) X(f)
$$

and is equal to the Fourier transform of the autocorrelation function. The cross power spectral density of two signals is given by

$$
G_{x y}(f)=X^{*}(f) Y(f)
$$

and is likewise the Fourier transform of the cross correlation function. The transfer function which relates the amplitude and phase of the components of the record $Y(f)$ to those of $\mathrm{X}(\mathrm{f})$ is determined by [58]

$$
h(f)=\frac{G_{x y}(f)}{G_{x x}(f)}
$$

with the associated coherence function given by

$$
\gamma_{x y}^{2}(f)=\frac{\left|G_{x y}(f)\right|}{G_{x x}(f) G_{y y}(f)} .
$$

For the interferometer signals considered in these experiments, having a small number of modulation periods spanning the record, the spectral density function is dominated by a single component at the modulation frequency. Given the special nature of these signals, we see that the most accurate determination of the spectral function results when the entire signal length is analyzed at once and no summation of frequency bins is performed.

\section{F.3 Methodology}

An array of complex numbers of the form

$$
N_{i} e^{j \phi_{i}}=4 h_{i} \sqrt{G_{x x}}
$$

is obtained form the data where $N_{i}$ is the amplitude of the ith periodic frequency component of the chord, while $\phi_{i}$ is the phase of the component relative to that of the 
reference chord, denoted as signal $\mathbf{x}$. The central chord is used as the reference because it typically has the highest signal level and is least affected by other systematic errors such as those from refraction, uncertainty in position, etc. Since plasma geometry can vary somewhat from shot to shot the array of complex chord density values is inverted to determine the corresponding densities as a function of the flux surface radius. Results from several shots are summed. In this way systematic errors resulting from intershot variation of the plasma position are minimized. The outer boundary condition is obtained by adding an additional point with a minor radius equal to 1.12 times that of the outermost chord. The real and imaginary density modulation components are set equal to zero here. The complex density coefficients for each chord are separated into real and imaginary components and then fit with a cubic spline. A set of equations with a path length matrix DY, which relates the local and line integrated density coefficients, is produced in a manner analogous with the approach outlined in Chapter 3. This overdetermined system is solved using singular value decomposition, which is equivalent to determining the least-squares solution to the equations [59]. Singular value decomposition is especially useful for diagnosing ill-conditioned matrices. The system of equations described above was, however, designed to be well-conditioned. One reason for using an over determined system is to avoid the numerical instability which exists in an uniquely determined system if the rank of the square matrix is less than its dimension. The array of $\tilde{n}(\rho)$ is used in the continuity equation cast in integral, spectral form,

$$
\int_{0}^{\rho} i \omega \tilde{n}(\rho \prime, \omega) \rho^{\prime} d \rho^{\prime}=\frac{1}{\rho}\left(\hat{D}(r) \frac{\partial \tilde{n}(\rho, \omega)}{\partial \rho}+\hat{V} \tilde{n}(\rho, \omega)\right)
$$

with the perturbed particle source term neglected in the interior region considered. The local values of flux surface radii $\rho$ and density-dependent variables are obtained from averaging 4 adjacent values, as described in Appendix C. This results in equations for $\mathrm{D}(\rho)$ and $\mathrm{V}(\rho)$ at 12 radial positions, similar to the number of chords available for the measurement. At each radial position equation F.10 is split into real and imaginary components. The resulting $(2 \times 2)$ matrix equations are solved for the transport coefficient values. 


\section{F.4 Complementary Spectral Analysis Method}

A complementary spectral method of analysis was also implemented. In this case the chord data was spline fit for each time step, then inverted using the same matrix inversion technique described earlier. The perturbed flux is determined from the integral form of the continuity equation in the time domain. Local values of these variables obtained from averaging over groups of four adjacent radial values are then Fourier analyzed. The perturbed flux

$$
\tilde{\Gamma}(r, \omega)=D(r) \frac{\partial \tilde{n}}{\partial r}(r, \omega)+V(r) \tilde{n}(r, \omega)
$$

is correlated with $\partial \tilde{n} / \partial r$ and $\tilde{n}$ using multidimensional spectral analysis through the equation [58]

$$
\mathbf{G}_{\mathbf{x y}}^{\mathrm{T}}=\mathbf{G}_{\mathbf{x x}}(\mathbf{V}, \mathbf{D})^{\mathrm{T}} .
$$

The principal difference between this method and the method of the preceding section is in the error propagation due to the different nature of the signal used for spline fits. Results of analysis of periodic modulation signals simulated at MTX chord positions are compared with the analytic functional forms of $\mathrm{D}, \mathrm{V}$ in fig. F.3. These profiles are similar to the results obtained from the other methods. This is typically the case when either simulated or experimental periodic modulation data are analyzed. 

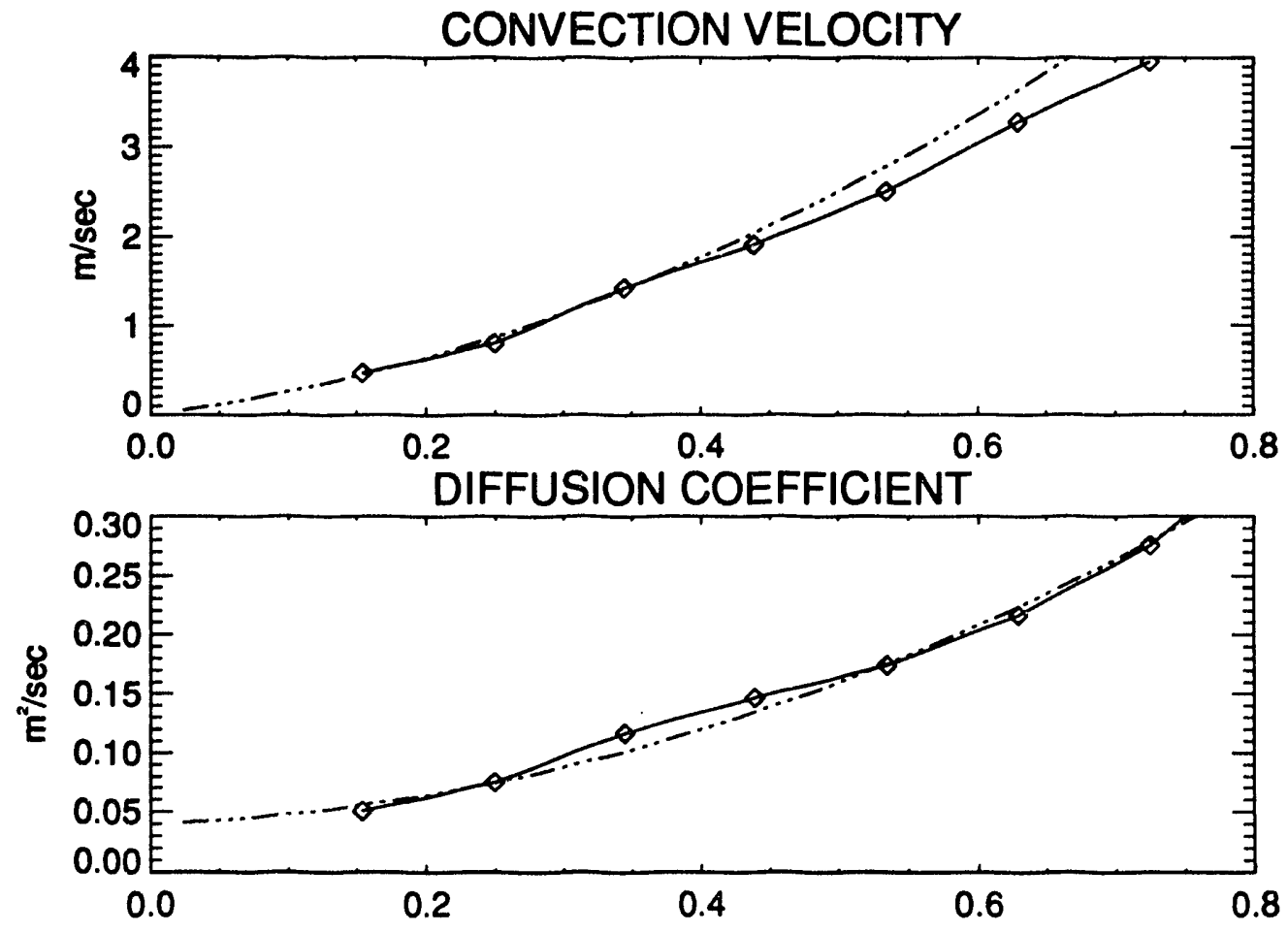

Figure F.3: The results of analysis of data generated at MTX chord positions with complementary spectral analysis method. 


\section{Appendix G}

\section{Accuracy of Fit}

The analysis presented in this section will demonstrate that the overall uncertainty in values of the transport coefficients is dominated by the systematic errors. Therefore it is not possible to assign exact limits to the overall uncertainty. This is often the case in experimental plasma physics, particularly with transport-related measurements. Reasonable estimates of the uncertainties in the transport coefficient values due to these systematic effects are obtained by examining the changes in the coefficient values as parameters in the fit routine are varied over relevant ranges of uncertainty. This will be discussed later later in this section. First, the relevance of the model used for the flux itself will be examined with several statistical tests.

\section{G.1 Statistical Tests of Models}

The validity of the results presented depends on the assumption that the linear model of equation 2.2 , which is of the form

$$
y=a_{1} x_{1}+a_{2} x_{2}+\epsilon,
$$

is appropriate to describe the relation between the perturbed quantities. In this section statistical evidence is presented which shows that this relation is appropriate. In addition to the above assumption of linearity, formulas for confidence intervals and statistical hypothesis tests for the results of linear regression analysis depend on three 
"inference" assumptions [64]. These are listed below:

- 1. Constant Variance - different populations of potential values of the dependent variable corresponding to different values of $\mathrm{x}$ have equal variances

- 2. Independence - any one value of the dependent variable is statistically independent of any other value of this variable

- 3. Normality - For any value $x_{i}$ of the independent variable $x$ the corresponding values of the dependent variable has a normal distribution

Since a low pass filter has been applied to the FIR data to remove periodic components, points in the filtered record separated by a time small compared to the inverse of the filter cutoff frequency are not statistically independent. Thus for the purposes of calculating confidence intervals and conducting statistical tests, we use a subset of the data points in the filtered records, separated by equal time steps of 6 msec. The differences between the values of the independent variables and values resulting from the least squares fit are called the residuals. If the time ordered residuals are not independent, then they are termed autocorrelated.

The Durbin-Watson test [64] is used to detect first-order autocorrelation in the data. The Durbin-Watson static is defined as

$$
d=\frac{\sum_{t=2}^{n}\left(e_{t}-e_{t-1}\right)^{2}}{\sum_{t=1}^{n} e_{t}^{2}}
$$

where $e_{1}, e_{2}, \ldots, e_{n}$ are the time-ordered residuals. It has been shown that values denoted $\mathrm{d}_{U, \alpha}$ exist such that if $d>d_{U, \alpha}$ the hypothesis that the error terms are not autocorrelated is accepted with probability 1- $\alpha$.

As described earlier, records were obtained for each data point by averaging over all of the shots in the group. When a subset of the points in the record, taken at 6 msec intervals, was used the Durbin-Watson test indicated the residuals were not autocorrelated with greater than $99 \%$ certainty in every instance. This is plausible since the low pass filter transfer function amplitude decays toward zero over a range of values well above the cutoff frequency. The presence of spectral components of the 

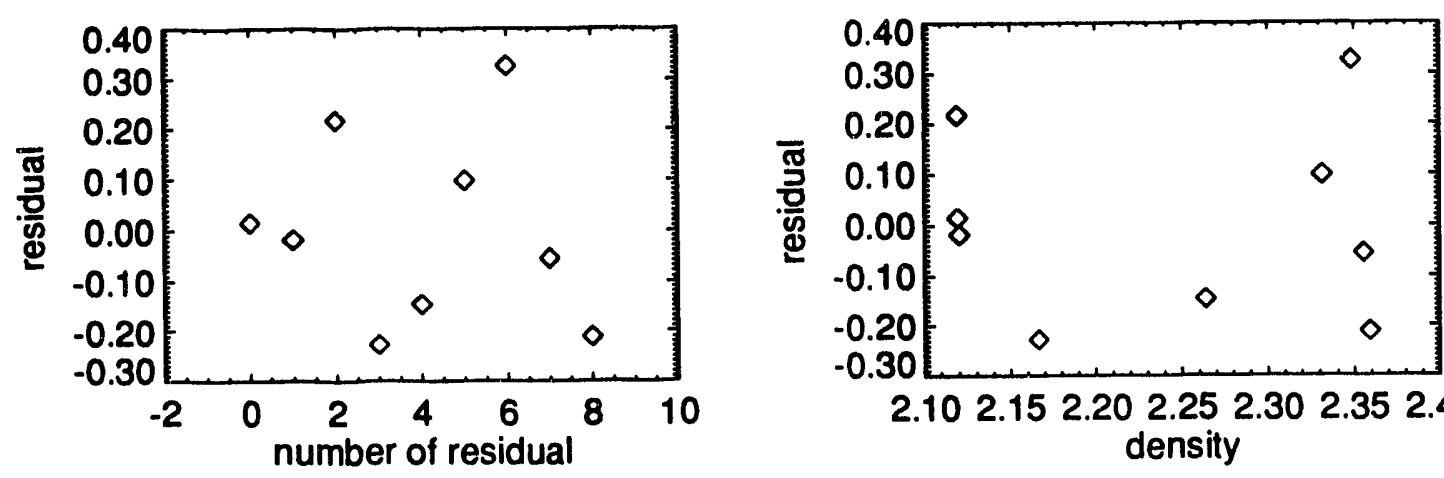

2.102 .152 .202 .252 .302 .352 .40 density
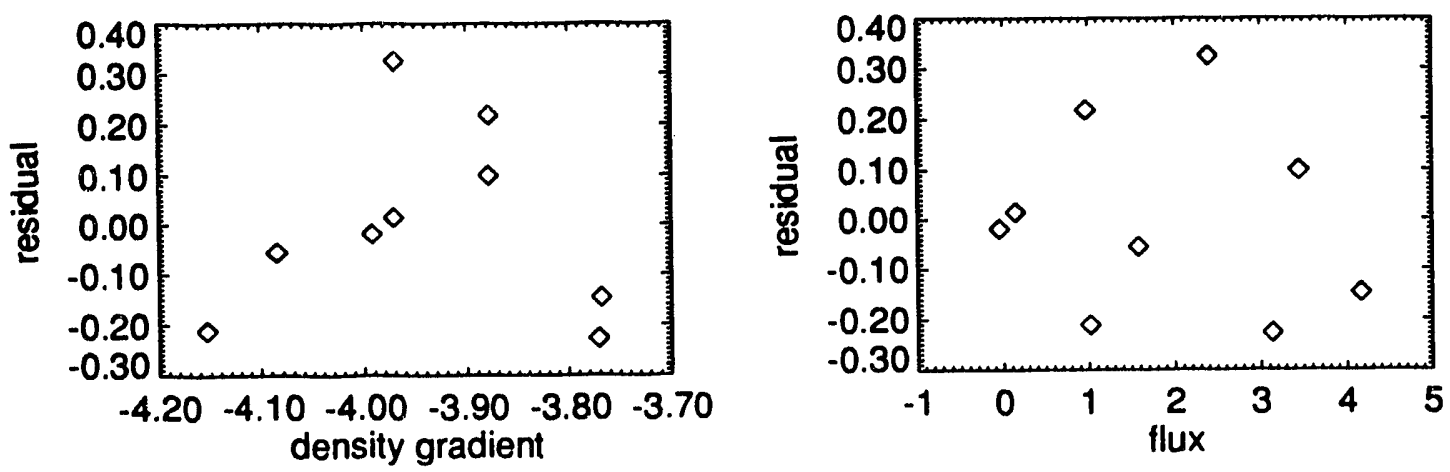

Figure G.1: Representative plots of residuals from fit versus other variables.

signal at frequencies well above the cutoff frequency allows values taken at $6 \mathrm{msec}$ intervals to be statistically independent.

The constant variance assumption is tested with the use of plots of residuals versus dependent or independent variables, or versus the time order in which they were collected. Representative plots are presented in figure G.1 for modulation data at $\rho=.8$ from plasma with $\bar{n}_{e, 20}=2.6, \mathrm{I}_{p}=285 \mathrm{kA}$. No trend is apparent, indicating constant variance, a result which is almost always true in the data.

The third assumption of normality is aptly examined here with the use of a normal plot. With this technique, discussed by Anscombe and Tukey [66], the residuals $e_{i}$ are ordered from smallest to largest and plotted against $z_{i}$. Here, $z_{i}$ is defined to be the location on abscissa of the standard normal curve so that the area under this curve to the left is $(3 i-1) /(3 n+1)$. When the assumption of normality holds and if the model has the correct functional form, then the plot should have the appearance of a straight line. Substantial variations from the straight-line appearance indicate a violation of the normality assumption [66]. A result for gas modulation data is plotted in fig. G.2 


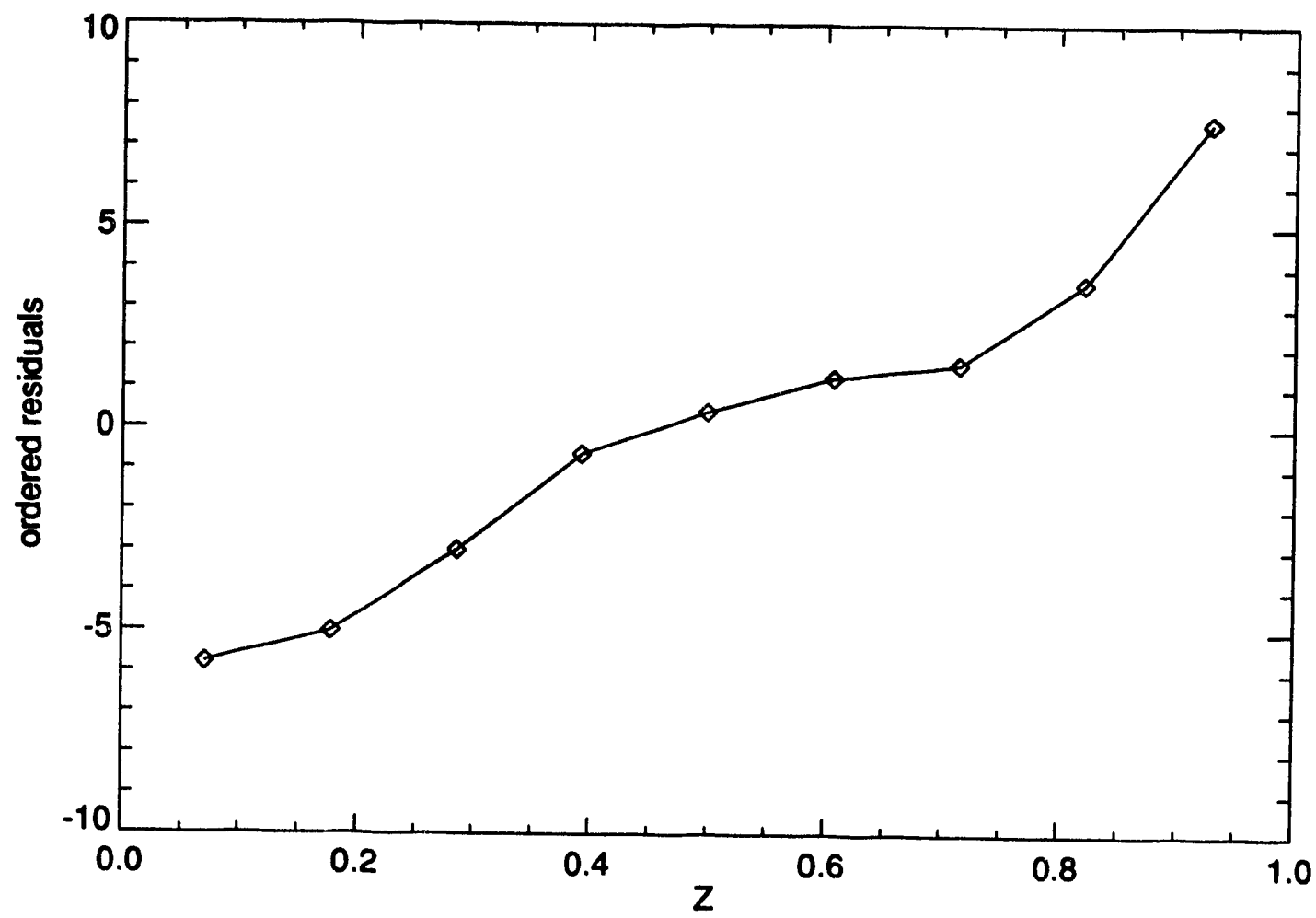

Figure G.2: Normal plots for fit to $\bar{n}_{e, 20}=2.6, \mathrm{I}_{p}=285 \mathrm{kA}$ data at $\rho=.55$.

for the same data considered above. This curve has a definite straight-line appearance indicating that the normality assumption approximately holds. Note the each data point resulted from averaging signals from 8-10 repeatable shots in order to obtain a good signal-to-noise ratio. The the total amount of information contained in the data is much greater than it might appear. In the regression analysis applied to gas modulation shots in TFTR, data from numerous reproducible shots was averaged for this same reason [67]. We see that the typical data analyzed with the multidimensional linear regression routine approximately satisfies the three inference assumptions.

\section{G.2 F-Test of the Model}

Now we consider the hypothesis that the independent variables in the model for the flux are related to the perturbed flux itself. Specifically we test the null hypothesis for the model $\Gamma=-D \nabla n+V n+\beta$

$$
\mathrm{H}_{0}: \mathrm{D}=\mathrm{V}=0
$$




\begin{tabular}{|cc|}
\hline normalized radius & F-statistic \\
\hline .076 & 2.13 \\
.198 & 2.83 \\
.320 & 5.61 \\
.442 & 14.8 \\
.564 & 36.1 \\
.686 & 26.5 \\
.808 & 6.85 \\
\hline
\end{tabular}

Table G.1: Values of F-statistic for model

which says that none of the independent variables $\tilde{n}, \nabla \tilde{n}$ affects $\tilde{\Gamma}$ versus the alternative hypothesis

$$
\mathrm{H}_{1} \text { : at least one of } \mathrm{D}, \mathrm{V} \neq 0 \text {. }
$$

We use the overall F-statistic [64] calculated from the linear regression fit. We can reject $\mathrm{H}_{0}$ in favor of $\mathrm{H}_{1}$ with probability $1-\alpha$ if $\mathrm{F}$ (model) $>F_{\alpha}^{(k-1, n-k)}$. The quantity $\mathrm{F}_{\alpha}^{(k-1, n-k)}$ is defined as the point on the scale of the F-distribution having $\mathrm{k}-1$ and $\mathrm{n}-\mathrm{k}$ degrees of freedom such that the area under the curve to the right of it is $\alpha$ [64]. For the model here $k=3$ and $n$ is the number of data points. In the data at $285 \mathrm{kA}$ the F-statistic indicates a definite relation between dependent and independent variables $\tilde{n}, \nabla \tilde{n}$ at the local position at greater than the $99 \%$ confidence level in most cases. Most of the remaining cases indicate a positive relation at the $95 \%$ confidence level. Values of the F-statistic for $\bar{n}_{e, 20}=1.8, \mathrm{I}_{p}=285 \mathrm{kA}$ fit at relevant radii are presented in table G.1. The values of the F-statistic for $95 \%$ and $99 \%$ confidence levels are 4.74 and 9.55 respectively.

The multiple correlation coefficient is a measure of how well the model fits the data. The square of the multiple correlation coefficient is defined as

$$
R^{2}=\frac{\text { Explained variation }}{\text { Total variation }} \text {. }
$$

For the $285 \mathrm{kA}$ data, values of $R$ at various radii range typically from 0.8 to 0.99 . In fig. G.3 the empirical flux is compared with the fit from the linear relationship at $\rho$ $=0.65$ for $\bar{n}_{e, 20}=2.2, \mathrm{I}_{p}=285 \mathrm{kA}$. A multiple correlation coefficient in excess of 0.5 


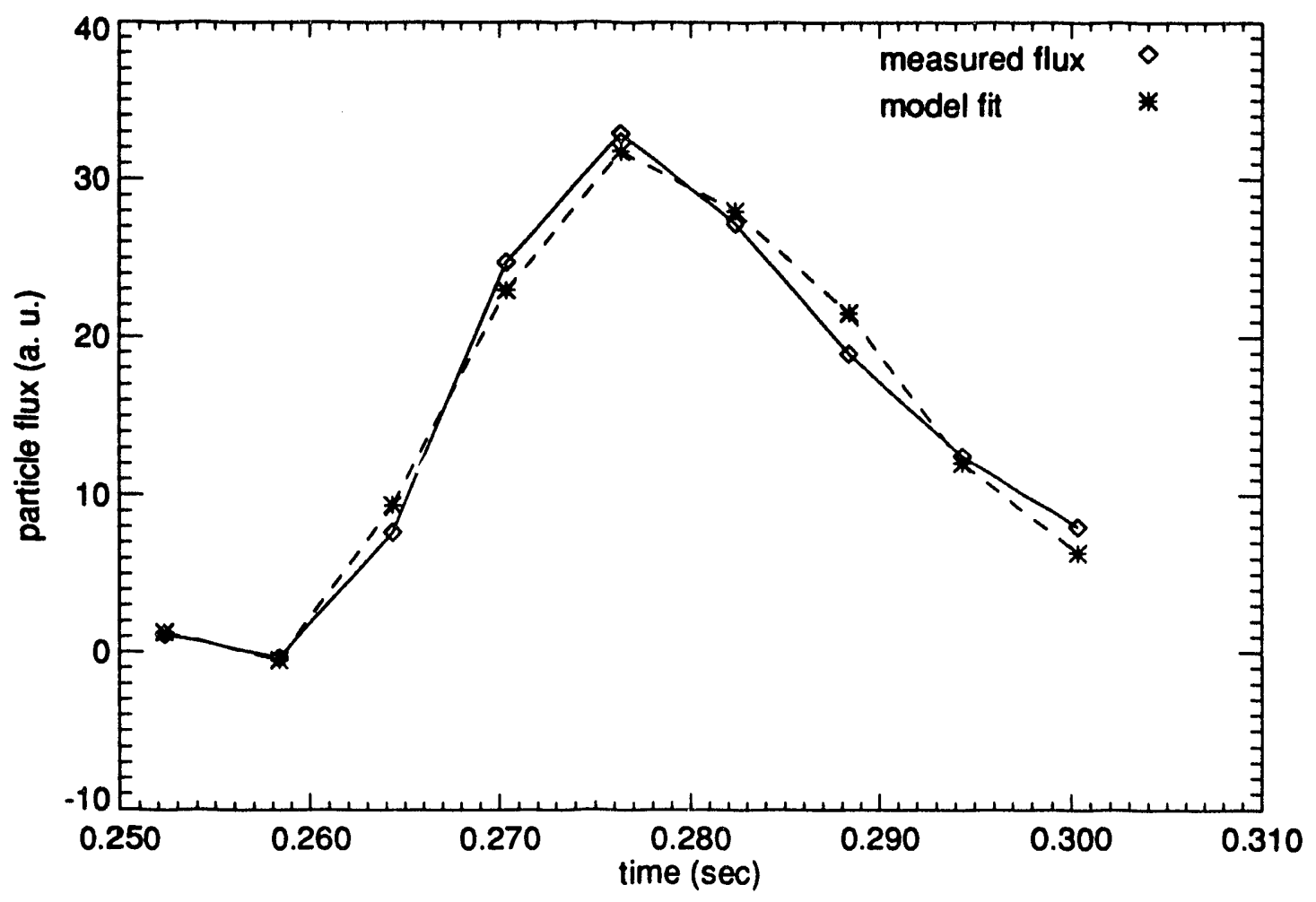

Figure G.3: Comparison of empirical flux with linear fit.

is considered necessary for the linear regression assumptions to hold [58]. It is not always possible to obtain a good regression fit to the data at every relevant radial position. For example, inside the region $\mathrm{r} / \mathrm{a}<0.2$ the overall integrated flux is small so that the signal-to-noise ratio is often poor.

In multidimensional linear regression analysis there is no requirement that the independent variables be mutually uncorrelated [58]. A practical requirement is that the correlation coefficient between independent variables not be close to unity, otherwise the matrix used in the linear regression analysis becomes nearly singular. If the independent variables are only moderately correlated the multidimensional linear regression operator is a powerful tool for determining the linear relation between the independent variables and the dependent variable. The statistical portion of the uncertainty in the transport coefficients is almost always a rather small fraction of the coefficient value. The exceptions occur near the center of the plasma $(r / a<0.2)$ where the transport coefficient values are usually smallest and the signal-to-noise ratio is lowest. Also mixing due to sawteeth influences transport there. Typical values 


\begin{tabular}{|ccc|}
\hline normalized radius & $\mathrm{V}(\mathrm{m} / \mathrm{sec})$ & $\mathrm{D}\left(\mathrm{m}^{2} / \mathrm{sec}\right)$ \\
\hline .076 & $.63 \pm .15$ & $.02 \pm .016$ \\
.198 & $.51 \pm .13$ & $.052 \pm .010$ \\
.320 & $.44 \pm .12$ & $.047 \pm .009$ \\
.442 & $.60 \pm .072$ & $.15 \pm .019$ \\
.564 & $.55 \pm .072$ & $.12 \pm .0122$ \\
.686 & $.57 \pm .061$ & $.20 \pm .0099$ \\
.808 & $1.8 \pm .067$ & $.27 \pm .0088$ \\
\hline
\end{tabular}

Table G.2: Transport coefficients and associated statistical uncertainties

of $\mathrm{V}$ and $\mathrm{D}$ and their associated $95 \%$ statistical confidence intervals versus radius are illustrated for the case of $\bar{n}_{e, 20}=2.6, \mathrm{I}_{p}=285 \mathrm{kA}$ in table G.2.

\section{G.3 Partial $F$-test}

The partial F-statistic is an indicator of whether individual independent variables in the model are significantly related to the dependent variable. Here we consider the two reduced models for the flux, $\tilde{\Gamma}=-D \nabla \tilde{n}$ and $\tilde{\Gamma}=-V \tilde{n}$. Residuals from fits obtained with the above reduced models and from the composite model are used to calculate partial F-statistics for each reduced model. If the partial F-statistic is greater than $\mathrm{F}_{\alpha}^{(p-g, n-k)}$ then at least one of the variables excluded in the reduced model is related to the dependent variable at the 1- $\alpha$ confidence level [64]. The quantity $\mathrm{F}_{\alpha}^{(p-g, n-k)}$ is the same function as was described in relation to the F-statistic, but with $\mathrm{p}-\mathrm{g}$, the number of variables in the composite model excluded from the reduced model, equal to one here.

In table G.3 partial F-statistics for each model are shown as functions of radius for $\bar{n}_{e, 20}=2.2, \mathrm{I}_{p}=285 \mathrm{kA}$. The values of $\mathrm{F}_{\alpha}^{1, n-k}$ for the $95 \%$ and $99 \%$ confidence levels are 5.59 and 12.25 respectively. It is clear from the example of table G.3 that both $\tilde{n}$ and $\nabla \tilde{n}$ are significantly related to $\tilde{\Gamma}$. Table G.4 lists the values of the multiple correlation coefficients for the reduced and composite models for the flux at various 


\begin{tabular}{|ccc|}
\hline normalized radius & $\Gamma=-\mathrm{D} \nabla \mathrm{n}$ & $\Gamma=-\mathrm{V} \mathrm{n}$ \\
\hline .076 & 13.5 & 3.79 \\
.198 & 42.6 & 30.3 \\
.320 & 13.0 & 11.9 \\
.442 & 14.5 & 20.2 \\
.564 & 5.21 & 5.00 \\
.686 & 5.80 & 4.02 \\
.800 & 59.6 & 55.8 \\
\hline
\end{tabular}

Table G.3: F-statistic values for the reduced models versus radial position.

\begin{tabular}{|cccc|}
\hline normalized radius & $\mathrm{R}(\Gamma=-\mathrm{D} \nabla \mathrm{n}-\mathrm{Vn})$ & $\mathrm{R}(\Gamma=-\mathrm{D} \nabla \mathrm{n})$ & $\mathrm{R}(\Gamma=-\mathrm{V} \mathrm{n})$ \\
\hline .076 & .844 & .741 & .836 \\
.198 & .951 & .873 & .828 \\
.320 & .977 & .960 & .894 \\
.442 & .934 & .773 & .796 \\
.564 & .796 & .702 & .639 \\
.686 & .893 & .821 & .513 \\
.800 & .992 & .634 & .450 \\
\hline
\end{tabular}

Table G.4: Correlation coefficients for the reduced and composite models versus radius.

radii for the plasma with $\bar{n}_{e, 20}=2.6, \mathrm{I}_{p}=285 \mathrm{kA}$. The accuracy of the overall fit is substantially reduced when either of the reduced models is used.

\section{G.4 Error Propagation}

Having established that the model for the flux is a statistically valid description which fits the data reasonably well, we can now consider the question of the accuracy of the results obtained. As demonstrated in the previous section, the statistical contribution to the uncertainty in the transport coefficients is typically quite small. . The particle source term is highly localized to the edge in these plasmas and is not a significant 
source of error in the region treated. The relative importance of the effect of low pass filtering on the signals was discussed in chapter 3 . It was shown that this effect is less significant than the error introduced through the spline fit for the values of transport coefficients found in MTX.

Dominant contributions to the error arise from factors such as uncertainty in the plasma position and geometry and uncertainties necessarily introduced through the spline fit. As evidenced in table 4.1 the changes in the plasma horizontal position from shot to shot are well quartified. Since the uncertainties in absolute plasma position are larger they will be the basis for estimating the consequent effect on the transport coefficients.

The sensitivity of the values of transport coefficients obtained to uncertainties in plasma position and geometry was examined by introducing artificial offsets to the values of fit paranneters which characterized the background fit, then applying the time domain analysis routine to the biased data. The three principal fit parameters used in the time dependent analysis routine are the plasma offset $\delta_{o}$, the Shafronov shift $\epsilon_{o}$ and the flux surface geometry parameter $\alpha$ in eq. 3.3. The Shafronov shift used was obtained from an equilibrium fit to polarimeter, interferometer and other shot data using the large aspect ratio expansion of the Grad-Shafronov equation [55]. The large aspect ratio expansion is an excellent approximation for the low $\beta_{p o l} \sim .3$ 5 plasmas with nearly circular flux surfaces produced in MTX. Over the range of currents and line-averaged densities at which gas modulation experiments were performed the values of the Shafronov shift at the center of the plasma relative to that at the last closed flux surface determined by the code varied by a rather modest amount. The value used in the analysis of all shot data here is $\epsilon_{\circ}=-0.0825=1.36$ $\mathrm{cm}$, determined for a typical gas modulation shot. An estimate of the un :ertainty of $\epsilon_{o}$ is $.015(0.25 \mathrm{~cm})$. This value is much greater than the standard deviation of values of $\epsilon_{o}$ determined from the equilibrium fit to polarimeter and other data for a variety of the plasmas in which gas modulation experiments were performed.

Values of $\epsilon_{o}$ were calculated for a range of profiles of electron temperature and 
density of the form

$$
\begin{aligned}
& T_{e}(r)=T_{e 0} e^{-\left(\frac{r}{a_{t}}\right)^{2}} \\
& n_{e}(r)=n_{e 0}\left(1-\left(\frac{r}{a}\right)^{2}\right)^{\beta}
\end{aligned}
$$

using the large aspect ratio expansion of the Grad-Shafronov equation. In Alcator $\mathrm{C}$ the empirical relation describing the width of the Gaussian fit to the electron temperature profile is

$$
a_{t}^{2}=\left(\frac{3 q_{o}}{2 q_{a}}\right) a^{2}
$$

with $\mathrm{q}_{o}$ is the safety factor at the center of the plasma and $\mathrm{q}_{a}$ is the value at the limiter. The density gradient scale lengths are considerably longer than those of the electron temperature profile, so that they value of the density profile exponent $\beta$ does not have much of an impact upon the value of the Shafronov shift parameter $\epsilon_{o}$ calculated. Over the range of values of $\mathrm{I}_{p}, \mathrm{a}_{t}$ and $\mathrm{n}_{e 0}$ found in the gas modulation data the values of $\epsilon_{o}$ resulting from this calculation varied from 1.2 to $1.7 \mathrm{~cm}$. These profiles of the Shafronov shift are well approximated by the function $\epsilon_{o}\left(1-\rho^{\alpha}\right)$ with $\alpha \simeq 2.75$. For all of the values of current, $a_{t}$ and $n_{e 0}$ covered in the gas modulation run the calculated profile shapes fit well within a region defined by the above function evaluated for $\alpha=2.25$ and 3.25. Therefore the value of uncertainty in $\alpha$ is put at \pm .5 .

The uncertainty in the plasma offset is taken to be $0.4 \mathrm{~cm}$. This is close to the maximum discrepancy in offset determined from polarimeter and interferometer data. When the offset parameter alone is varied by such a modest amount the analysis routine adjusts the size scale of the grid of plasma flux surfares. Normalized radial positions $\rho$ at which transport coefficient values are calculated shift accordingly. Although the calculated values change as their positions shift, they vary in such a way so that values obtained at the original "unbiased" radii through interpolation change relatively little. Over the rar, ge of uncertainty considered here, the most pronounced effect variation in this parameter has is to cause some expansion or contraction in the radial scale of coefficient profiles. 
The overall effect of these systematic uncertainties on the transport coefficients is assessed by adding offsets to the values of $\delta_{o}, \alpha$ and $\epsilon_{o}$ simultaneously. These offsets vary over the range of uncertainties associated with each parameter as stated above. The same combination of offsets is used for each shot in the summation of data from all of the shots associated with a particular plasma state (i.e. $\bar{n}_{e}, \mathrm{I}_{p}$ ). In this way the induced error is systematic, yielding a conservative estimate.

The effects of the offsets added to the values of $\delta_{o}, \alpha$ and $\epsilon_{o}$ were examined for a number of such combinations varied through the stated ranges of uncertainty. The aggregate uncertainties in $\mathrm{D}(\mathrm{r})$ and $\mathrm{V}(\mathrm{r})$ result from the combination of systematic and random errors. Examples of these are shown in fig. 4.20. In the outer region of the plasma uncertainties in D of order $15 \%$ are not unusual. Near the plasma center, where the values of $\mathrm{D}$ are rather smaller, the fractional uncertainties can be quite substantial. When the values of coefficients cover a sizable range across the plasma cross section the smaller values cannot be accurately determined. Their value are simply localized to a small range. The fractional uncertainties in $V(r)$ are generally larger than those for $\mathrm{D}(\mathrm{r})$. This result was obtained even when analyzing simulated data having coefficient values like the ones determined for MTX plasmas.

The changes in the transport coefficients in the outer plasma near the density limit are clearly significant. The substantial difference between the plasma transport behavior in shots near the density limit and at lower densities was evident in the unprocessed central chord FIR signals shown in chapter 4, fig. 4.21 as well. 


\section{Appendix $\mathbf{H}$}

\section{Model with Temperature Effects Explicitly Included}

In principal the effects of the four perturbed quantities $(\tilde{n}, \nabla \tilde{n}, \tilde{T}, \nabla \tilde{T})$ could be separated by expressing the flux as

$$
\tilde{\Gamma}=\frac{\partial \Gamma}{\partial n} \tilde{n}+\frac{\partial \Gamma}{\partial \nabla n} \nabla \tilde{n}+\frac{\partial \Gamma}{\partial T} \tilde{T}+\frac{\partial \Gamma}{\partial \nabla T} \nabla \tilde{T}
$$

and performing a four dimensional regression correlation. In practice, since the temperature and density perturbations of the slow wave are coupled, it is not possible to produce perturbations to these variables $(\tilde{n}, \tilde{T})$ which are uncorrelated using a single gas puff in MTX.

When independent variables in the data are highly correlated, difficulties arise in the linear regression analysis. The term which has been coined in the literature to describe this is multicollinearity $[64,68,69]$. The linear regression model can be written in matrix notation as $\mathbf{y}=\mathbf{X} \beta+\epsilon$ with

$$
y=\left[\begin{array}{c}
y_{1} \\
y_{2} \\
\vdots \\
y_{n}
\end{array}\right] \quad \beta=\left[\begin{array}{c}
\beta_{1} \\
\beta_{2} \\
\vdots \\
\beta_{n}
\end{array}\right]
$$




$$
x=\left[\begin{array}{ccccc}
1 & x_{11} & x_{12} & \ldots & x_{1 k} \\
1 & x_{21} & x_{22} & \ldots & x_{2 k} \\
\vdots & \vdots & \vdots & \ddots & \vdots \\
1 & x_{n 1} & x_{n 2} & \ldots & x_{n k}
\end{array}\right]
$$

where $\mathrm{n}$ is the number of observations and $\mathrm{k}$ is the number of independent variables. The regression coefficients are obtained simply from

$$
\hat{\boldsymbol{\beta}}=\left(\mathbf{X}^{\mathrm{T}} \mathbf{X}\right)^{-1} \mathbf{X}^{\mathrm{T}} \mathbf{y} .
$$

A high degree of correlation between independent variables in the model causes the $\mathbf{X}^{\mathrm{T}} \mathbf{X}$ matrix to become nearly singular. In this case small variations in the individual values of the independent variables can cause large changes in the coefficients obtained through multidimensional linear regression analysis. The coefficient values are then subject to large errors.

The degree of multicollinearity present can be diagnosed by taking the ratio of the largest to smallest eigenvalues of the matrix $\mathbf{X}^{\mathrm{T}} \mathbf{X}$ to determine its condition number [69]. If the condition number is less than 100 there is generally no serious problem with multicollinearity [69]. Condition numbers between 100 and 1000 signal moderate to strong multicollinearity. Values in excess of 1000 indicate severe multicollinearity.

Temperature and density data from the $285 \mathrm{kA}$ density scan are correlated with $\Gamma$ according to the relation of H.1 for $0<\rho<0.65$. Values of condition numbers obtained for these data sets with the four dimensional correlation range isom $\sim 100$ to nearly 1000 , with the great majority near the lower end of this range. Values of $\partial \Gamma / \partial \nabla n$ obtained are generally quite similar to values of $\mathrm{D}$ obtained when temperature effects were included implicitly rather than explicitly. This holds true in particular when condition numbers are in the low 100's. The values of the other coefficients vary wildly due to the effects of multicollinearity.

\section{H.1 Biased Estimators}

The only type of method suited for dealing with multicollinearity applicable here is the biased estimator technique. Ridge regression, principal components regression and 
other biased estimator techniques are discussed in detail in the literature $[64,68,69]$. They are considered controversial. Each method has its supporters and opponents. The purpose of the application here is to examine the behavior of the coefficients as bias is introduced into the estimation procedure. We will consider the principal components regression method here.

In the principal components model

$$
y=\mathbf{Z} \alpha+\epsilon
$$

where $\mathbf{Z}=\mathbf{X} \mathbf{T}, \alpha=\mathbf{T}^{\mathrm{T}} \beta$ and $\mathbf{T}^{\mathrm{T}} \mathbf{X}^{\mathbf{T}} \mathbf{X} \mathbf{T}=\mathbf{Z}^{\mathrm{T}} \mathbf{Z}=\mathbf{\Lambda}$, with $\boldsymbol{\Lambda}$ a diagonal matrix of the eigenvalues of $\mathbf{X}^{\mathrm{T}} \mathbf{X}$ and $\mathbf{T}$ an orthogonal matrix whose columns are the eigenvectors associated with the eigenvalues. The columns of $\mathbf{Z}$ define a new set of orthogonal regressors which are referred to as principal components. In principal components regression the principal components which correspond to near zero eigenvalues are removed and least squares analysis is applied to the remaining components. Gunst and Mason [70] compared results obtained when ridge regression, principal components regression, latent root and least squares estimators were applied to sizable amounts of numerically generated data with varying amounts of multicollinearity. Their results indicated that principal components regression yielded values of coefficients much more accurate than the least squares estimate when the data are ill-conditioned. Using the model of equation H.1, the principal components regression was applied to the $285 \mathrm{kA}$ data at $6 \mathrm{msec}$ intervals. The principal components curresponding to small eigenvalues, having values less than of order .01 that of the maximum eigenvalue were removed. For most of the data this corresponded to removing one of the principal components. Results obtained for $\partial \Gamma / \partial \nabla n$ with this method are very close to the values of $\mathrm{D}$ obtained when the $\mathrm{T}_{e}$ and $\nabla T_{e}$ were not explicitly included. $\mathrm{A}$ comparison is shown in fig. $\mathrm{H} .1$ for the plasma with $\bar{n}_{e}=2.2 \times 10^{20} \mathrm{~m}^{-3}$ and $\mathrm{I}_{p}=285$ $\mathrm{kA}$. This result is fully consistent with the derivation of sec. 2.2 which showed that the effect of off diagonal terms appears in $\mathrm{V}$. We recognize that $\mathrm{V}$ and $\partial \Gamma / \partial n$ are distinctly different quantities here.

Estimates of the values of $\partial \Gamma / \partial n$ obtained with principal components regression 


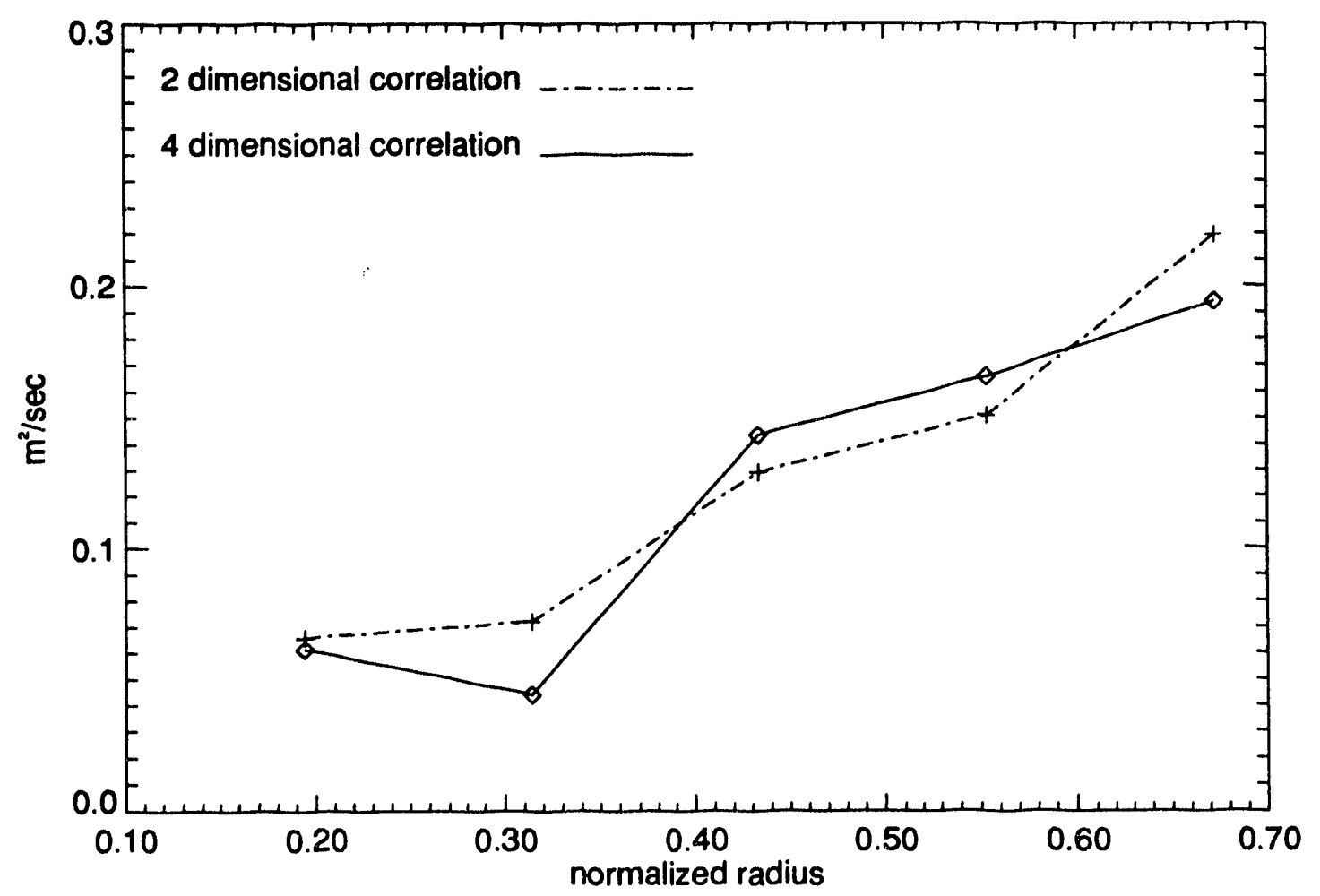

Figure H.1: Comparison of perturbed diffusion coefficients obtained with implicit or explicit consideration of $\mathrm{T}_{e}$ perturbations. 


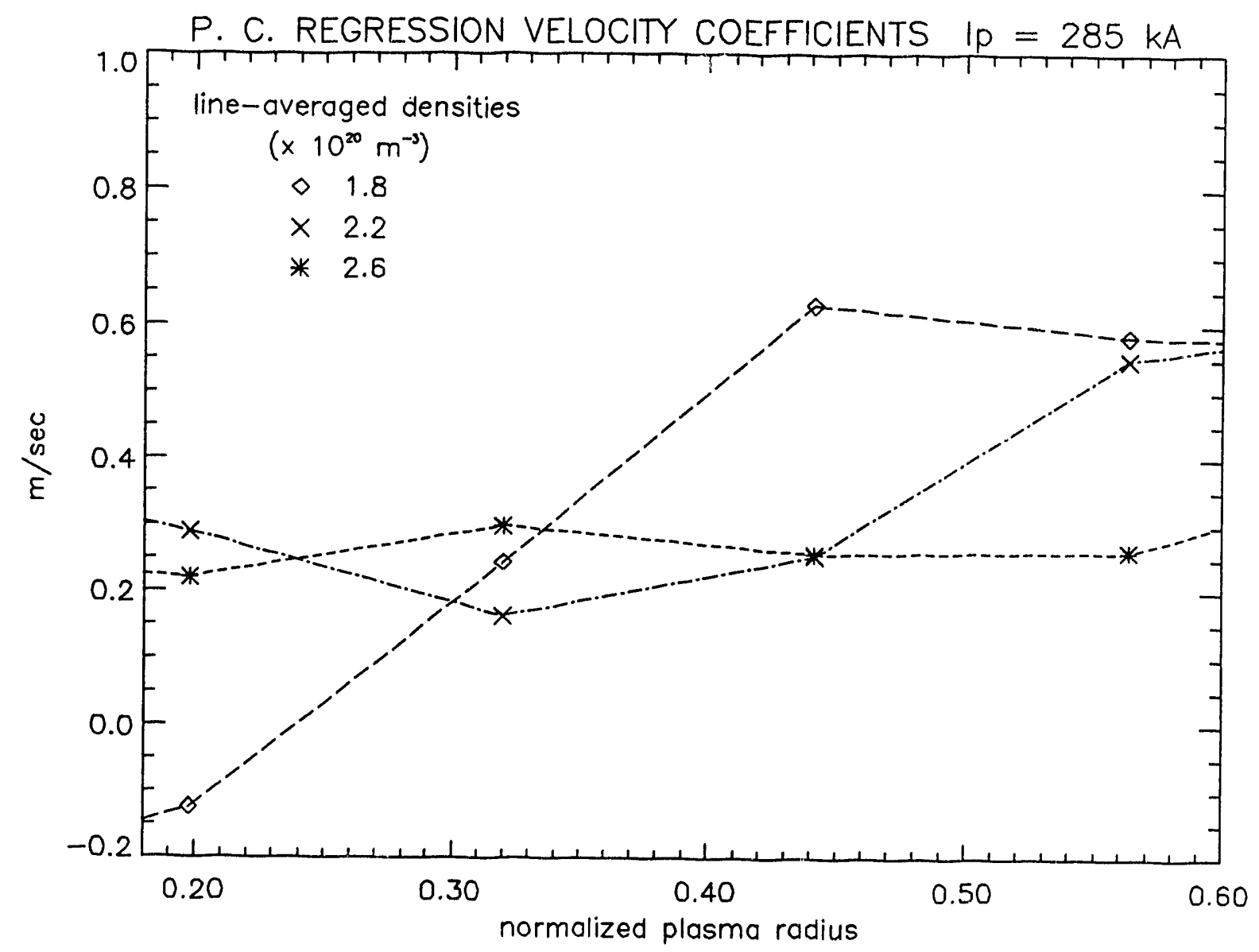

Figure H.2: Estimates of $\frac{\partial \Gamma}{\partial n}$ obtained from p. c. regression

are shəwn in fig. H.2, for $0<\rho<0.65$, for cases where the multicollinearlity due to the inclusion of the $T_{e}, \nabla T_{e}$ data was moderate, not severe. Values obtained are of the same sign as obtained for $\mathrm{V}$ earlier. They are generally somewhat smaller than those for $\mathrm{V}$ but usually not by more than a factor of 2 .

\section{H.2 Statistical Tests of Extended Models}

In past sections we used the partial F-test to determine whether individual variables were significantly related to the dependent variable. Multicollinearity can cause the partial F-test to yield misleading indications about the importance of independent variables [64]. We must examine other methods of assessing the significance of the 
model fit to the data.

One measure used earlier was the multiple correlation coefficient, $R$. The addition of any independent variable to a regression model will increase the multiple correlation coefficient and decrease the sum of the squares of the residuals. This does not mean that the new model is a better description of the relation between independent and dependent variables. The multiple correlation coefficient $\mathrm{R}$ will always increase, even if the variable added is unimportant.

Another criterion for comparing separate regression models is known as the $\mathrm{C}$ statistic. This is often called the $\mathrm{C}_{k}$ statistic in the statistical literature. Consider a set of $\mathrm{p}$ potential independent variables. If we choose a regression model based upon a subset of these variables

$$
y=\beta_{o}+\beta_{1} x_{1}+\ldots+\beta_{k-1} x_{k-1}+\epsilon
$$

then the $\mathrm{C}$ statistic for the model is defined as

$$
C=\frac{S S E}{s_{p}^{2}}-(n-2 k) .
$$

with the unexplained variation $\mathrm{SSE}=\sum_{i=1}^{n}\left(y_{i}-\hat{y}_{i}\right)^{2}$, and $\mathrm{k}$ the total number of parameters in the model. Here $n$ is the number of observations and $s_{p}$ is the mean square error calculated from the model

$$
y=\beta_{o}+\beta_{1} x_{1}+\ldots+\beta_{p} x_{p}+\epsilon
$$

which contains all $\mathrm{p}$ independent variables and thus $p+1$ parameters, i.e.

$$
s_{p}^{2}=\frac{S S E}{n-(p+1)}
$$

We want $\mathrm{C}$ to be small. If an unimportant variable is added to the model $\mathrm{C}$ can increase. According to the theory behind the $\mathrm{C}$ statistic we wish to find a model for which the $C$ statistic roughly equals $k$, the number of parameters in the model. When a model has a $\mathrm{C}$ statistic substantially greater than $\mathrm{k}$, it can be shown that the model has has substantial bias [64]. We consider to what extent we are biasing the results of the regression analysis by using only the density-related independent 


\begin{tabular}{|lllll|}
\hline normalized radius & $\bar{n}_{e, 20}=2.9$ & $\bar{n}_{e, 20}=2.6$ & $\bar{n}_{e, 20}=2.2$ & $\bar{n}_{e, 20}=1.8$ \\
\hline .073 & 4.0 & 3.1 & 5.8 & 2.2 \\
.190 & 4.4 & 1.1 & 1.9 & 2.7 \\
.306 & 3.4 & 2.3 & 2.3 & 5.2 \\
.540 & 1.3 & 2.2 & 1.3 & 2.0 \\
.657 & 11.8 & 2.5 & 1.1 & 1.9 \\
\hline
\end{tabular}

Table H.1: Values of $\mathrm{C}$ statistic at various line densities and radii

variables. Table $\mathrm{H} .1$ gives values of the $\mathrm{C}$ statistic at various radii for several cases calculated for the (unbiased) linear regression model using $(n, \nabla n)$ from the set of variables $(\mathrm{n}, \nabla \mathrm{n}, \mathrm{T}, \nabla \mathrm{T})$. Again data is used at 6 msec intervals. In most of the cases the $\mathrm{C}$ statistic is less than or not substantially greater than $\mathrm{k}=3$. Thus the reduced model is not significantly biased. Comparing $\mathrm{C}$ statistics for models containing both density-related variables and one temperature-related variable, we find the case where $\nabla \mathrm{T}$ is excluded usually has a lower value of $\mathrm{C}$ than the case where $\mathrm{T}$ is excluded. This implies that $\mathrm{T}$ is more closely correlated with $\Gamma$ in this data than $\nabla \mathrm{T}$.

Since $\tilde{T}$ is highly correlated with $\tilde{n}$, the information contained in $\tilde{T}$ is redundant. We conclude that, at least to within our ability to measure the $T_{e}$ profile, we can use a model for $\Gamma$ in the regression analysis which excludes an explicit dependence on $\mathrm{T}_{e}$ and $\nabla T_{e}$ without introducing significant bias. The effect of these terms becomes manifest in the perturbed convection velocity $\mathrm{V}$. 


\section{Appendix I}

\section{Outline of}

\section{Resistivity-Gradient-Driven}

\section{Turbulence Theories}

A summary of the published derivations of several versions of the RGDT theory [89, $90,92,42,115]$ is included for reference in this appendix. The theoretical model considered is based upon the reduced resistive magnetohydrodynamic (MHD) equations in cylindrical geometry [91]. These are

$$
\begin{aligned}
\rho \frac{d \mathbf{V}}{d t} & =-\nabla p+\mathbf{J} \times \mathbf{B} \\
\mathbf{E}+\mathbf{V} \times \mathbf{B} & =\eta \mathbf{J} \\
\nabla \times \mathbf{B} & =\mu_{o} \mathbf{J} \\
\nabla \cdot \mathbf{B} & =0
\end{aligned}
$$

If beta is small, $\beta \ll\left(a / R_{o}\right)^{2}$, the spatial variation in the toroidal field due to diamagnetic effects in the resulting ordering is also small. Thus the equations, in cylindrical geometry, reduce to

$$
\begin{aligned}
E_{\|} & =\frac{\partial \psi}{\partial t}-B_{z} \nabla_{\|} \phi=\eta J_{z} \\
\frac{d U}{d t} & =B_{z} \nabla_{\|} J_{\|}
\end{aligned}
$$


with $\psi$ the poloidal flux function (=-R $\left.\mathrm{A}_{\phi}\right)$ and $\phi$ the fluid stream function $\left(=\Phi / B_{z}\right)$, where $\Phi$ is the electrostatic potential. The current density is given by

$$
J_{z}=\frac{1}{\mu_{o}} \nabla_{\perp}^{2} \psi
$$

and $U$ is the component of vorticity in the $z$ direction

$$
U=\nabla_{\perp} \cdot\left(\rho_{m} \nabla_{\perp} \phi\right)
$$

Here $\rho_{m}$ is the mass density and the coordinate $\mathrm{z}$ is in the direction of the cylinder axis. An additional equation describes resistivity evolution. In its most basic form it is

$$
\frac{d \eta_{s p}}{d t}=\nabla_{\|} \chi_{\|} \nabla_{\|} \eta_{s p}
$$

In this equation $\chi_{\|}$is the parallel electron thermal diffusivity. The total convective derivative and parallel gradient are

$$
\begin{aligned}
\frac{d}{d t} & \equiv \frac{\partial}{\partial t}+\left(\nabla_{\perp} \phi \times \hat{z}\right) \cdot \nabla \\
B_{z} \nabla_{\|} & \equiv\left(\nabla_{\perp} \psi \times \hat{z}\right) \cdot \nabla+B_{z} \frac{\partial}{\partial z} .
\end{aligned}
$$

Equations I.5-I.9 are the basis for the resistivity-gradient-driven turbulence model. By using the electrostatic approximation

$$
\begin{aligned}
\nabla_{\|} & =\nabla_{\|}^{(0)}=\hat{b} \cdot \nabla \\
\tilde{\psi} & =0
\end{aligned}
$$

(with $\hat{b}$ parallel to the field) and linearizing the equations they can be reduced to

$$
\begin{aligned}
-B_{z} \nabla_{\|}^{(0)} \phi & =\tilde{\eta} J_{z 0}+\eta_{o} \tilde{J}_{z} \\
\rho_{m} \frac{d}{d t} \nabla_{\perp}^{2} \phi & =B_{z} \nabla_{\|}^{(0)} \tilde{J}_{z} \\
\frac{d \tilde{\eta}}{d t}-\chi_{\|} \nabla^{(0 ;, 2} \tilde{\eta} & =-\frac{1}{r} \frac{\partial \phi}{\partial \theta} \frac{\partial \eta_{0}}{\partial r} .
\end{aligned}
$$

The current perturbation can now be eliminated resulting in two nonlinear equations for $\phi$, and $\eta_{s p}$

$$
\begin{aligned}
\frac{\rho_{m}}{B_{z}^{2}} \frac{d}{d t} \nabla_{\perp}^{2} \phi & =-\frac{1}{\eta_{0}} \nabla_{\|}^{(0), 2} \phi-\frac{J_{z 0}}{B_{z}} \nabla_{\|}^{(0)}\left(\frac{\tilde{\eta}}{\eta_{0}}\right) \\
\frac{d \tilde{\eta}}{d t}-\chi_{\|} \bar{\nabla}_{\|}^{(0), 2} \tilde{\eta} & =-\frac{1}{r} \frac{\partial \phi}{\partial \theta} \frac{d \eta_{o}}{d r} .
\end{aligned}
$$


Equations I.17, I.18 are used as the model for resistivity-gradient-driven turbulence calculations.

Two energy-like quantities are identified which are quadratic in the fluctuation level. These are the fluid kinetic energy

$$
E_{K}=\frac{1}{2} \int d^{3} x\left|\nabla_{\perp} \phi\right|^{2}
$$

and the mean square resistivity fluctuation level

$$
E_{T}=\frac{1}{2} \int d^{3} x|\tilde{\eta}|^{2}
$$

From equations $1.17, \mathrm{I} .18$ it was shown that these satisfy the following evolution equations

$$
\begin{aligned}
\frac{\partial E_{K}}{\partial t} & =\frac{B_{z}^{2}}{\rho_{m}} \int d^{3} x\left[\frac{\phi^{*} J_{z 0}}{B_{z 0}} \nabla_{\|}^{(0)}\left(\frac{\tilde{\eta}}{\eta_{0}}\right)-\frac{\left|\nabla_{\|}^{(0)} \phi\right|^{2}}{\eta_{0}}\right] \\
\frac{\partial E_{T}}{\partial t} & =\int d^{3} x\left(-\tilde{\eta}^{*} \frac{1}{r} \frac{\partial \phi}{\partial \theta} \frac{d \eta_{0}}{d r}-\chi_{\|}\left|\nabla_{\|}^{(0)} \tilde{\eta}\right|^{2}\right) .
\end{aligned}
$$

The fluid kinetic energy $\mathrm{E}_{k}$ evolves by a competition between a destabilizing $\mathbf{J} \times \mathbf{B}$ force induced by resistivity perturbations and a stabilizing $\mathbf{J} \times \mathbf{B}$ force induced by the magnetic field line diffusion [89]. The mean square resistivity fluctuation level $\mathrm{E}_{T}$ is driven by relaxation of the average resistivity gradient and damped by dissipation due to $\chi_{\|}$.

The nonlinear saturation condition is given by

$$
\frac{\partial E_{K}}{\partial t}=\frac{\partial E_{T}}{\partial t}=0
$$

The requirement $\partial E_{K} / \partial t=0$ is equivalent to imposing the criterion that [89]

$$
\int d^{3} x \phi^{*} \nabla_{\|}^{(0)} \tilde{J}_{z}=0
$$

A sufficient, but not necessary, condition for satisfying the latter criterion is $\tilde{J}_{z}=$ 0 . This condition is satisfied approximately except in the immediate vicinity of the rational surface on which the mode in located. The $\tilde{\eta}$ and $\tilde{\phi}$ eigenfunctions, which are skewed off symmetry about the mode resonant surface in the linear theory, are 
broadened by turbulent diffusion as the fluctuation level increases [89]. Thus the region located at the singular surface is irrelevant to the nonlinear dynamics of rippling mode turbulence.

Because of the $\tilde{J}_{z}=0$ condition the vorticity equation decouples from the system, resulting in considerable simplification. The radial mode structure of the saturated turbulence is determined by a balance of thermal convection with diffusion and not by the linear eigenmode width. Thus the saturated state with $\tilde{J}_{z}=0$ and a nonlinear scale length is radically different in character from the linear regime [89]. By taking an asymptotic balance of thermal conduction with diffusion an expression for the radial scale for $\tilde{\eta}_{k}$ was obtained

$$
\Delta_{k}^{c}=\left(D_{k} / \chi_{\|} k_{\|}^{\prime 2}\right)^{\frac{1}{4}}
$$

where

$$
k_{\|}=k_{\|}^{\prime} x=\frac{k_{\theta}}{L_{s}} x=\frac{m}{L_{s} r_{s}} x .
$$

The subscript $\mathrm{k}$ denotes the combination of poloidal and toroidal mode numbers $(\mathrm{m}, \mathrm{n})$. The quantity $\Delta_{k}^{c}$ is an amplitude-dependent mixing length which adjusts so that the source and sink terms balance. From the second saturation condition, a balance between parallel thermal conduction and the resistivity gradient driving term yields

$$
\Delta_{k}^{c} \approx\left(\frac{L_{s} E_{0}}{L_{\eta} B_{z}}\right)^{\frac{1}{3}}\left(\chi_{\|} k_{\|}^{\prime 2}\right)^{-\frac{1}{3}}
$$

By eliminating $\Delta_{k}^{c}$ from the two relations one obtains

$$
D_{k} \approx\left(\frac{L_{s} E_{0}}{L_{\eta} B_{z}}\right)^{\frac{4}{3}}\left(\chi_{\|} k_{\|}^{\prime^{2}}\right)^{-\frac{1}{3}}
$$

At saturation damping occurs when a resistivity fluctuation parcel is scattered to finite $\mathrm{x}$ by radial diffusion and then destroyed by thermal dissipation, proportional to $\chi_{\|}\left(k_{\|}^{\prime} \Delta_{k}^{c}\right)^{2}$. We observe that the saturated mixing length and associated diffusion coefficient are rather weakly dependent upon $\chi_{\|}$in equations I.27 and I.28.

Average levels of turbulent fluctuating quantities are estimated from nonlinear theory. The mean square radial velocity for the system is ca'culated as $[89,94]$

$$
\left(\tilde{V}_{r}\right)_{r m s} \approx 1.23\left(\frac{L_{s} F_{0}}{L_{\eta} B_{z}}\right)
$$




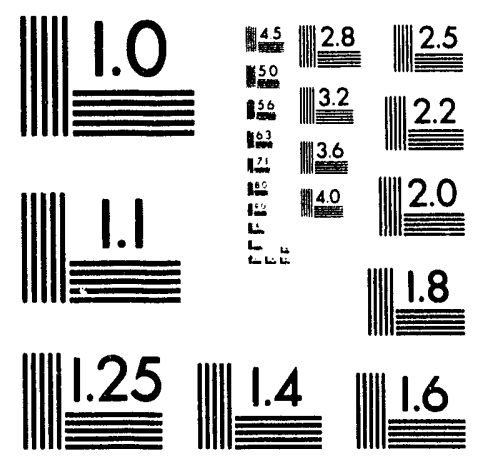



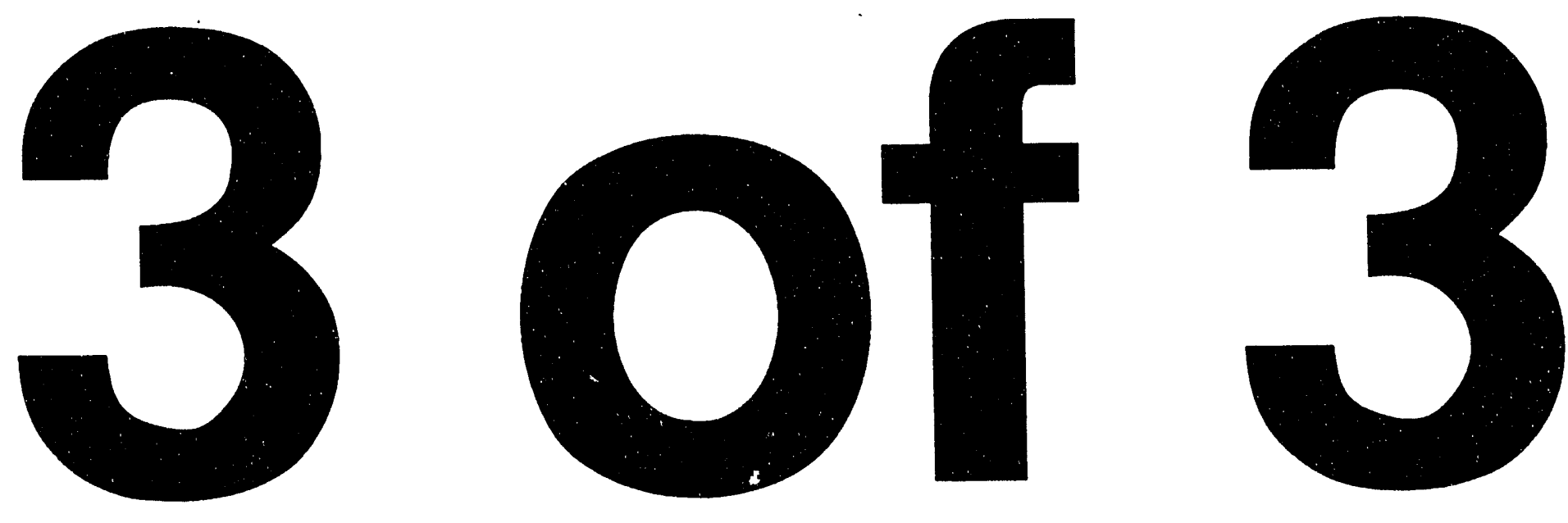
Analytic scaling laws derived from nonlinear theory were compared with results of numerical studies. The nonlinear equations I.5- I.9 were solved by a three-dimensional initial value code. All of the numerical results showed very clear evidence of a nonlinear saturation mechanism. The fluctuation and transport levels compared favorably with the scalings of the analytic expressions.

\section{I.1 RGDT with Impurity Dynamics Included}

A version of the theory was developed which included the effect of impurity dynamics [90]. A single low $\mathrm{Z}$ impurity species was considered. An equation describing the evolution of $\mathrm{Z}_{\text {eff }}$ was derived from a model given by Rutherford [95]. This equation is

$$
\frac{d Z_{\text {eff }}}{d t}=\nabla_{\|} \chi_{z} \nabla_{\|} Z_{\text {eff }}
$$

where $\chi_{z}=V_{t h, i}^{2} / Z^{2} \nu_{i i}$ is the effective transport coefficient for impurity-ion flow along field lines, resulting from the balance of the parallel impurity pressure gradient with impurity-ion friction. The derivation of the theory proceeds along the same lines as presented in the last section. The principal difference is that resistivity fluctuations in this case are driven primarily by fluctuations in $Z_{\text {eff }}$. Therefore this mode is governed by impurity ion flow along field lines proportional to $\chi_{z} \nabla_{\|} Z_{\text {eff }}$. Since $\chi_{z} \ll \chi_{\|}$the impurity gradient driven modes are significantly more robust than their temperature gradient driven analogs.

In the published results expressions for fluctuation and transport levels are derived from nonlinear theory and are in good agreement with the results of numerical calculation [90]. The turbulent particle flux is given by

$$
\Gamma_{n}=\frac{n_{0}}{L_{n}}\left(\frac{E_{0} L_{s}}{B_{z} L_{\eta}}\left(1+\eta_{z}\right)\right)^{\frac{4}{3}}\left(\chi_{z} \bar{k}_{\|}^{2}\right)^{-\frac{1}{3}}
$$

with $L_{n}^{-1}=-\frac{1}{n_{0}} \frac{d n_{0}}{d r}, \eta_{z}=L_{\eta} / L_{z}$ and $E_{0}, B_{z}$ the toroidal electric and magnetic fields respectively. Since the particle flux is linear in $\nabla n$ the equilibrium and perturbed 
diffusion coefficients are equal. The diffusion coefficient is

$$
\left.D_{n}=\Gamma_{n} \frac{L_{n}}{n_{0}}=\left(\frac{E_{0} L_{s}}{B_{z} L_{\eta}}\left(1+\eta_{z}\right)\right)^{\frac{4}{3}}\left(\chi_{z} \bar{k}_{\|}\right)^{2}\right)^{-\frac{1}{3}}
$$

which scales as

$$
D_{n} \sim T^{-5 / 6} n^{1 / 3}\left(L_{\eta}^{-1}+L_{z}^{-1}\right)^{4 / 3} L_{s}^{2} B_{z}^{-4 / 3} V_{L}^{4 / 3} r_{s}^{2 / 3} R_{0}^{4 / 3} .
$$

with the shear length defined as $\mathrm{L}_{s}=\mathrm{Rq} / \hat{s}$.

For typical tokamak profiles this diffusion coefficient increases with radius. It also scales as $\mathrm{D} \sim I_{p}^{2} \sim q^{2}$ so that particle confinement should improve with increasing current; conversely, this transport mechanism would be enhanced in plasmas with high q.

The particle flux carries a convective thermal flux given by

$$
Q=T_{0} \Gamma_{n}\left(1+\eta_{e}\left(\frac{\chi z}{\chi_{T}}\right)^{1 / 3}\right) .
$$

The calculated magnetic fluctuation levels $\left(\tilde{B}_{r} / B_{z}\right)_{r m s}$ are quite small so that the mode is predominantly electrostatic. The associated thermal diffusivity decreases with radius and the corresponding diffusive thermal transport is insignificant. Thus the model predicts that thermal transport in a cool, highly resistive edge plasma is caused primarily by convection [90].

Results from TEXT' indicate that a very high fraction of particle flux at the plasma edge, if not all, is driven by electrostatic turbulent fluctuations [96]. Measurements of the electrostatic fluctuation driven particle fluxes at the plasma edge in the Caltech [97], Tosca [98] and TEXT [99] and DITE [100] tokamaks indicate they can account for a large fraction of the total thermal flux. In TEXT the electrostatic portion of the energy flux dominates at the edge for all but the lowest densities [111].

\section{I.2 Neoclassical Resistivity-Gradient-Driven Tur- bulence}

The resistivity-gradient-driven turbulence models considered so far have been based upon reduced equations of resistive magnetohydrodynamics which were derived for a 
plasma in the Pfirsch-Schlüter collisionality regime. A version of the rippling theory based upon neoclassical MHD equations [114] was developed [115]. In this theory of neoclassical resistivity-gradient-driven turbulence the rippling modes couple to the density gradient through the dependence of neoclassical resistivity on density. The neoclassical resistivity can be written as $[114,116]$

$$
\eta_{n c}=\eta_{s p}\left[1+4.51 \epsilon^{1 / 2} /\left(1+1.02 \nu_{* e}^{1 / 2}+1.07 \nu_{* e}\right)\right]
$$

where $\nu_{* e}=\nu_{e} / \omega_{b e} e^{3 / 2}$. Thus the structure of the neoclassical resistivity fluctuation can be written as

$$
\frac{\tilde{\eta}_{n c}}{\eta_{n c}}=-C_{t} \frac{\tilde{T}_{e}}{T_{e}}-C_{n} \frac{\tilde{n}}{n_{0}}
$$

where

$$
C_{n}=\frac{\partial \ln \eta_{n c}}{\partial \ln n_{o}}=\frac{4.51 \epsilon^{1 / 2}\left(0.51 \nu_{* e}^{1 / 2}+1.07 \nu_{* e}\right)}{\left(1+1.02 \nu_{* e}^{1 / 2}+1.07 \nu_{* e}\right)^{2}}\left(1+\frac{4.51 \epsilon^{1 / 2}}{1+1.02 \nu_{* e}^{1 / 2}+1.07 \nu_{* e}}\right)^{-1}
$$

and

$$
C_{t}=-\frac{\partial \ln \eta_{n c}}{\partial \ln T_{e}}=\frac{3}{2}-2 C_{n} .
$$

The density coupling coefficient to the neoclassical resistivity has a maximum value around $\nu_{* e} \sim 1$ and vanishes in the limits $\nu_{* e} \rightarrow 0$ or $\nu_{* e} \rightarrow \infty$. The equilibration of density fluctuations is affected by collisions between trapped and untrapped particles. This has a much weaker stabilizing effect than thermal conduction. Therefore the region where resistivity-gradient-driven turbulence is significantly excited broadens inward from the edge of the plasma to the region where $\nu_{* e} \leq 1$ [115]. 


\section{Bibliography}

[1] K. B. Axon, G. A. Baxter, J. Burt et al. in Plasma Physics and Controlled Nuclear Fusion Research, Proc. 7th Int. Conf. Innsbruck, IAEA, Vienna, Vol. 1 (1979) 51.

[2] L. A. Beiry, C. E. Bush, J. D. Callen et al., in Plasma Physics and Controlled Nuclear Fusion Research, Proc. 6th Int. Conf. Berchtesgaden, IAEA, Vienna (1977) 49.

[3] The Jet Team, Plasma Phys. and Contr. Fusion 32 (1990) 1083.

[4] Yu. Yu. Karzhavin, R. D. Bengtson, D. L. Brower, Y. Jiang, G. X. Li, H. Y. W. Tsui, Effect of Neon Injection On Turbulence In TEXT-U, Bull. Amer. Phys. Society, Nov. 1992. 37(6), 2 P21.

[5] A. J. Wootton, B. A. Carreras, H. Matsumoto, K. McGuire, et al.,Phys. Fluids B2 (1990) 2879.

[6] Yu. N. Dnestrovskij, S. V. Neudachin, G. V. Pereverzev, Sov. Journal Plamsa Phys. 10 (1984) 137.

[7] A. D. Cheetham, A. Gondhalekar, J. C. M. De Haas O. N. Jarvis, P. D. Morgan, et al., in Plasma Physics and Controlled Fusion Research, (Proc. 12th Int. Conf., Nice, 1988), Vol. I, IAEA (1989) 483.

[8] L. R. Baylor, W. A. Houlberg, S. L. Milora, et al., Nuclear Fusion 31(7) (1991) 1249 .

[9] V. A. Vershkov, S. V. Mirnov, Nuclear Fusion 14 (1974) 383.

[10] Y. Kamada, N. Hosogane, R. Yoshino, Nuclear Fusion 31 (1991) 1827.

[11] P. E. Scott, Nuclear Fusion 28 (1988) 1469.

[12] S. Ortolani, G. Rostagni, Nuclear Instruments and Methods 207 (1983) 35.

[13] K. Borrass, Y. L. Igitkhanov, N. A. Uckan, Report of the ITER Experts' Meeting on Density Limits, ITER-TN-PH-9-6.

[14] K. Borrass, Nuclear Fusion 31(6) (1991) 1035. 
[15] F. W. Perkins, R. A. Hulse, Phys. Fluids 28 (1985) 1837.

[16] M. Greenwald, J. L. Terry, S. M. Wolfe et al., Nuclear Fusion 28 (1988) 2199.

[17] M. Greenwald, M. Besen, F. Camacho, et al., in Plasma Physics and Controlled Nuclear Fusion Research 1986, (Proc. 11th Int. Conf., Kyoto, 1986) Vol. 1, IAEA, Vienna (1987) 139.

[18] A. Gibson, Nuclear Fusion 16 (1976) 546.

[19] J. Allen, G. E. Austin, K. B. Axon, et al., in Plasma Physics and Controlled Nuclear Fusion Research 1986, (Pıoc. 11th. Int. Conf. Kyoto, 1986), Vol. 1, IAEA, Vienna (1987) 227.

[20] The Jet Team, Plasma Physics and Controlled Fusion 32 (1990) 958.

[21] T. Yu. Akatova, L. G. Askinasi, et al., in Plasma Physics and Controlled Nuclear Fusion Research (Proc. Thirteenth Int. Conf., Washington, 1990), Vol. I, IAEA, Vienna (1991) 509.

[22] V. V. Alikaev, A. A. Bagdasarov, A. A. Borshchegovskij, et al., Studies of the Density Limit and Related Phenomena in T-10 Tokamak, ITER-IL-PH-12-9-S-2.

[23] K. W. Gentle, B. Richards, F. Waelbroeck, Plasma Physics and Controlled Fusion 29 (1987) 1077.

[24] P. C. Efthimion, D. K. Mansfield, B. C. Stratton, et al., Phys. Re. Letters 66 (1991) 421.

[25] K. W. Gentle, B. Richards, M. E. Austin, Physical Review Letters 68 (1992) 2444 .

[26] K. W. Gentle, Phys. Fluids 31 (1988) 1105.

[27] H. H. Bell, A. L. Hunt, C. A. Clower, The Gas Box Control System for Tandem Mirror Experiment-Upgrade, LLNL UCRL Report \# 89231, (1983).

[28] Stanley, Dougherty, Dougherty, Digital Signal Processing, second edition, (Reston, Reston VA, 1984).

[29] K. Tani, M. Azumi, R. Devoto, JAERI-Memo 01-376, (1989).

[30] R. Devoto, LLNL UCRL Report \#102633 Rev. 3, (1991) (to be published in Nucl. Fusion).

[31] G. Arfken, Mathematical Methods for Physicists, Academic Press, San Diego (1985).

[32] Y. Yasutomo, K. Miyata, S. I. Himeno, T. Enoto, Y. Ozawa, IEEE Trans. on Plasma Science PS-9 (1982) 15. 
[33] V. V. Alikaev, A. A. Bagdasarov, G. A. Bobrovskij, A. A. Borshchegovskij, et al., in Plasma Physics and Controlled Nuclear Fusion Research (Proc. Thirteenth Int. Conf., Washington, 1990), Vol. I, IAEA, Vienna (1991) 163.

[34] A. Stabler, H. Niedermeyer, R. Lock, V. Mertens, E. R. Müller, F. X. R. Söldner, F. Wagner, et al. Controlled Fusion and Plasma Physics, Proc. 16th Eur. Conf Venice, European Physical Society, Part I, 13B (1989) 23.

[35] J. A. Wesson, et al., Nuclear Fusion 29 (1989) 641.

[36] B. B. Kadomtsev, Comments Plasma Phys. Controlled Fusion 13 (1989) 57.

[37] S. J. Fielding, J. Hugill, G. M. McCracken et al., Nuclear Fusion 17 (1977) 1382.

[38] M. Murakami, J. D. Callen, L. A. Berry, Nuclear Fusion 16 (1976) 34\%..

[39] T. Tsunematsu, J. Hogan, K. Borass, F. Engelmann, et al., Operational Limits and Disruptions in Tokamaks: Status and Application to ITER, ITER-TN-PH8-2.

[40] D. L. Brower, C. X. Yu, et al., Physical Review Letters, 67 (1991) 200.

[41] A. S. Ware, P. H. Diamond, B. A. Carreras, et al., Phys. Fluids B4 (1992) 102.

[42] D. R. Thayer, P. H. Diamond, Physical Review Letters 65 (1990) 2784.

[43] C. Hidalgo, C. P. Ritz, T. L., Rhodes W. L. Rowan, P. H. Diamond, et al., Nuclear Fusion 31(9) (1991) 1661.

[44] H. R. Fetterman, et al., Appl. Phys. Lett. 33 (1978) 151.

[45] J. P. Crenn, IEEE Trans. Microwave Theory Tech., MTT-26 (1979) 573.

[46] C. A. Brooksby, B. W. Rice, W. A. Peebles, Proc. IEEE 12th Symposium on Fusion Engineering, Monterey, CA, 1478 (1987).

[47] P. A. Pribyl, Ph.D. Thesis, Plasma Position Control On Alcator C MIT (1981).

[48] A. A. Bagdasarov, N. L. Vasin, V. A. Vershkov, et al., Plamsa Physics and Controlled Nuclear Fusion Research 1984, Vol. 1 (1984) 181.

[49] N. Gottardi, J. Appl. Phys. 50 (1979) 2647.

[50] H. K. Park, Plasma Physics and Controlled Fusion 31 (1989) 2035.

[51] A. R. Jacobsen, J. Appl. Phys. 53 (1982) 8549.

[52] Y. Nagayama, J. Appl. Phys. 62 (1987) 2702.

[53] C. Janicki, R. Decoste, C. Simm, Physical Review Letters 62 (1989) 3038. 
[54] B. LaBombard, Ph.D. Thesis, Poloidal Asymmetries in the Limiter Shadow Plasma of the Alcator C Tokamak MIT (1986).

[55] B. W. Rice, Ph.D. Thesis, Poloidal Magnetic Field Profiles Measurements on the Microwave Tokamak Experiment Using Far-Infrared Polarimetry LLNL (1992), also B. W. Rice, private communication.

[56] D. Véron, in Infrared and Millimeter Waves, edited by K. J. Button (Academic, New York, 1979) Vol. 2.

[57] P. R. Bevington, Data Reduction and Error Analysis for the Physical Sciences (McGraw-Hill, New York, 1969).

[58] J. S. Bendat, A. G. Piersol, Random Data: Analysis and Measurement Procedures, second edition, (Wiley, New York, 1987).

[59] W. H. Press, B. P. Flannery, L. A. Teukolsky, W. T. Vetterling, Numerical Recipies: The Art of Scientific Computing, (Cambridge, New York, 1986).

[60] F. Engelmann, M. Curatolo, Nuclear Fusion 13 (1973) 497.

[61] A. E. Costley, R. J. Hastie, J. W. M. Paul, J. Chamberlain, Physical Review Letters 33 (1974) 758.

[62] C. J. Lasnier, private communication

[63] B. Lipschultz, J. Nucl. Mat. 15 (1987) 145.

[64] B. R. Bowerman, R. T. O'Connell, Linear Statistical Models an Applied Approach, second edition, (PWS-KENT, Boston 1990).

[65] M. Born, E. Wolf, Principles of Optics, fifth edition, (Permagon, New York, 1975).

[66] F. J. Anscombe, J. W. Tukey, Technometrics 5 (1963) 141.

[67] P. C. Efthimion, M. Bitter, E. D. Fredrickson, R. J. Goldston, et al., Plamsa Physics and Controlled Nuclear Fusion Research, (Proc. 12th Int. Conf., Nice, 1988), Vol. I, IAEA (1989) 307.

[68] A. Sen, M. Srivastava, Regression Analysis: Theory, Methods and Applications, (Springer-Verlag, New York, 1990) 218.

[69] D. C. Montgomery, E. A. Peck, Introduction to Linear Regression Analysis, (Wiley, New York, 1982).

[70] R. F. Gunst, R. L. Mason, J. Amer. Statist. Assoc. 72 (1977) 616.

[71] Equipe TFR, Plasma Phys. 25 (1983) 641.

[72] R. L. Watterson, R. E. Slusher, C. M. Surko, Phys. Fluids 28 (1985) 2857. 
[73] E. Mazzucato, Phys. Rev. Letters 48 (1982) 1828.

[74] R. E. Slusher, C. M. Surko, Proceedings of the 1982 International Conference on Plasma Physics, (Chalmers Institute of Technology, Göteborg, Sweden, 1982), Paper10a:2, and K. McGuire and the PDX Group, Phys. Rev. Letters 50 (1983) 891, \& 51 (1984) 1925.

[75] S. J. Zweben, R. W. Gould, Nuclear Fusion 23 (1983) 1625.

[76] T. C. Simonen, D. L. Brower, P. C. Efthimion, et al., Comments on Plasma Physics and Controlled Fusion 14 (1991) 57.

[77] C. C. Gomez, Ph.D. Thesis, Study of Electron Temperature Evolution During Sawtoothing and Pellet Injection Using Thermal Electrom Cyclotron Emission in the Alcator C Tokamak MIT (1986).

[78] S. M. Wolfe, M. Greenwald, R. Gandy, et al., Nuclear Fusion 26 (1986) 329.

[79] A. A. Ware, Phys. Rev. Lett. 25 (1970) 115.

[80] K. W. Gentle, O. Gehre, K. Krieger, Nuclear Fusion 32 (1992) 217.

[81] R. S. Granetz, Physical Review Letters 49(9) (1982) 658.

[82] M. A. Makowski, Granetz Limit on MTX, LLNL M-Div. Memo, March 1990.

[83] S. J. Levinson, J. M. Beall, E. J. Powers, R. D. Bengtson, Nuclear Fusion 23 (1984) 527.

[84] Ch. P. Ritz, R. D. Bengtson, S. J. Levinson, E. J. Powers, Phys. Fluids 27 (1984) 2956.

[85] B. Coppi, M. N. Rosenbluth, R. Z. Sagdeev, Phys. Fluids 10 (1967) 582.

[86] N. Mattor, Transport from Ion Temperature Gradient Driven Turbulence, CLMP872.

[87] P. W. Terry, et al., Phys Fluids B1 (1989) 109.

[88] D. R. McCarthy, P. N. Gudzar, J. F. Drake, et al., Phys. Fluids 4 (1992) 1846.

[89] L. Garcia, P. H. Diamond, B. A. Carreras, J. D. Callen, Phys. Fluids 28 (1985) 2147.

[90] T. S. Hahm, P. H. Diamond, P. W. Terry, L. Garcia, B. A. Carreras, Phys. Fluids 30 (1987) 1452.

[91] B. V. Waddell, B. A. Carreras, H. R. Hicks, J. A. Holmes, Phys. Fluids 22 (1979) 896.

[92] D. R. Thayer, P. H. Diamond, Phys. Fluids 30 (1987) 3724. 
[93] H. R. Strauss, Phys. Fluids 20(8) (1977) 1354.

[94] P. W. Terry, P. H. Diamond, K. C. Shiang, L. Garcia, B. A. Carreras, Phys. Fluids 29 (1986) 2501.

[95] P. H. Rutherford, in Physics of Plasma Close to Thermonuclear Conditions, edited by B. Coppi, et al., (Permagon, New York, 1981), Vol. 1, 143.

[96] W. L. Rowan, et al., Nuclear Fusion 27 (1987) 1105.

[97] P. C. Liewer, J. M. McChesney, S. J. Zweben, R. W. Gould, Phys. Fluids 29 (1986) 309.

[98] A. Howling, A. Coté, E. J. Doyle, D. E. Evans, D. C. Robinson, in Proceedings of the 12th European Conference on Controlled Fusion and Plasma Physics, Budapest, Hungary, 1985, edited by L. Póas and A. Montavi, (European Physical Society, Budapest, 1985), Vol. I, 311.

[99] P. H. Diamond, J. F. Drake, H. Matsumoto, J. Sheffield, P. W. Terry, A. J. Wootton, Comments Plasma Phys. Controlled Fusion 13(6) (1990) 327.

[100] P. Mantica, et al., in Proceedings of the 16th European Conference on Controlled Fusion and Plasma Physics (European Physical Society, Venice, 1989) Vol. 3, 967.

[101] T. J. Dolan, Fusion Research, Vol. I, (Permagon Press, New York, 1982) 66.

[102] D. R. Thayer, private communication, also P. H. Diamond, private communication.

[103] P. W. Terry, private communication.

[104] C. Hidalgo, R. Balbin, M. A. Pedrosa, et al., Phys. Rev. Letters 69 (1992) 1205.

[105] A. S. Ware, P. H. Diamond, B. A. Carreras, J.-N. Leboeuf, D. K. Lee, Phys. Fluids B4 (1992) 102.

[106] A. S. Ware, P. H. Diamond, H. Biglari, B. A. Carreras, L. A. Charlton, J.-N. Leboeuf, A. J. Wooton, Phys. Fluids B4 (1992) 877.

[107] G. S. Lee, P. H. Diamond, Theory of Ion-Temperature-Gradient-Driven Turbulence, University of Texas, IFSR \#209, 1986.

[108] A. Boileau, H. W. H. Van Andel, M. von Hellermann, A. Rogister, Nuclear Fusion 27 (1987) 109.

[109] T. W. Petrie, A. G. Kellman, M. A. Mahdavi, Plasma Density Limits During Ohmic-, L-mode and Elming H-mode Operation in DIII-D, General Atomics Report GA-A19987, 1992, (to be published in Nucl. Fusion).

[110] Y. B. Kim, H. Biglari, P. W. Terry, Phys. Fluids B2 (1990) 2143. 
[111] Ch. P. Ritz, R. V. Bravenec, P. M. Schoch, R. D. Bengtson, et al., Physical Review Letters 62 (1989) 1844.

[112] Y. J. Kim, Ph.D. Thesis, General Description of Magnetic Fluctuations in TEXT, University of Texas, Austin, 1989.

[113] Ch. P. Ritz, T. L. Rhodes, H. Lin, et al., in Plasma Physics and Controlled Nuclear Fusion Research, Washington, 1990 (IAEA, Vienna, 1991), Vol. 2, 589.

[114] J. D. Callen, W. X. Qu, K. D. Siebert, B. A. Carreras, K. C. Shiang, D. A. Spong, in Plasma Physics and Controlled Fusion Research, 1986 (IAEA, Vienna, 1987) Vol. II, 157.

[115] O. J. Kwon, P. H. Diamond, T. S. Hahm, Phys. Fluids B1 (1989) 2172.

[116] F. L. Hinton, R. D. Hazeltine, Rev. Mod., Phys. 48 (1976) 237, and S. P. Hirshman, D. J. Sigmar, Nuclear Fusion 21 (1981) 1079.

[117] B. A. Carreras, P. W. Gaffney, H. R. Hicks, J. D. Callen, Phys. Fluids 25 (1982) 1231.

[118] H. P. Furth, J. Killeen, M. N. Rosenbluth, Phys. Fluids 6 (1963) 459.

[119] A. Rogister, Plamsa Phys. 28 (1986) 547.

[120] A. B. Hassam, J. F. Drake, The Rippling Instability, University of Maryland, Physics Publication Number 82-129, 1981.

[121] W. M. Tang, F. Romanelli, S. Briguglio, Phys. Fluids 31(10) (1988) 2951.

[122] M. E. Foord, E. S. Marmar, J. L. Terry, Rev. Sci. Instrum. 53(9) (1982) 1407.

[123] R. C. Isler, W. L. Rowan, W. L. Hodge, Phys. Rev. Lett. 55 (1985) 2413.

[124] C. M. Surko and R. E. Slusher, Science 221 (1983) 817.

[125] M. M. Pickrell, Ph.D. Thesis, The Role of Radiation on the Power Balance of the Alcator C Tokamak, MIT 1976.

[126] D. C. Robinson, Electrostatic Turbulence and Anomalous Transport, Culham CLM-P869, 1989.

[127] M. G. Bell, G. L. Schmidt, P. C. Efthimion, et al., Nuclear Fusion 32 (1992) 1585 .

[128] G. Rewoldt, W. M. Tang, Phys. Fluids B2 (1990) 318.

[129] P. N. Yushmanov, Contrib. Plasma Phys. 30 (1990) 139.

[130] A. Yu. Dnestrovskij, P. N. Yushmanov, Edge Plasma Transport Coefficient Study under Self-Consistent Tokamak Discharge Simulation, ITER PH15-SU-S1. 
[131] T. O. Ohkawa, Phys. Lett. 67A (1978) 35.

[132] R. E. Waltz, R. R. Dominguez, S. K. Wong, 11th Int. Conf. on Plasma Phys. and Contr. Fus. Research, Kyoto, 1986, A-VI-1-3.

[133] V. V. Parail, P. N. Yushmanov, JETP Letters 42 (1985) 278.

[134] Yu. N. Dnestrovskij, Yu. V. Esipchuk, K. A. Razumova, P. N. Yushmanov, "Investigation of Transport Processes Near the Density Simit", in Plasma Physics and Controlled Nuclear Fusion Research (Proc. Thirteenth Int. Conf., Washington, 1990), Vol. I, IAEA, Vienna (1991) 393.

[135] A. R. Polevoy, P. N. Yushmanov, Density Limit Simulation Based on T-10 Results, ITER-IL-PH-12-9-S-3.

[136] K. Borrass, D. J. Campbell, S. Clement, G. C. Vlases, Nuclear Fusion 33(1) (1993) 63. 

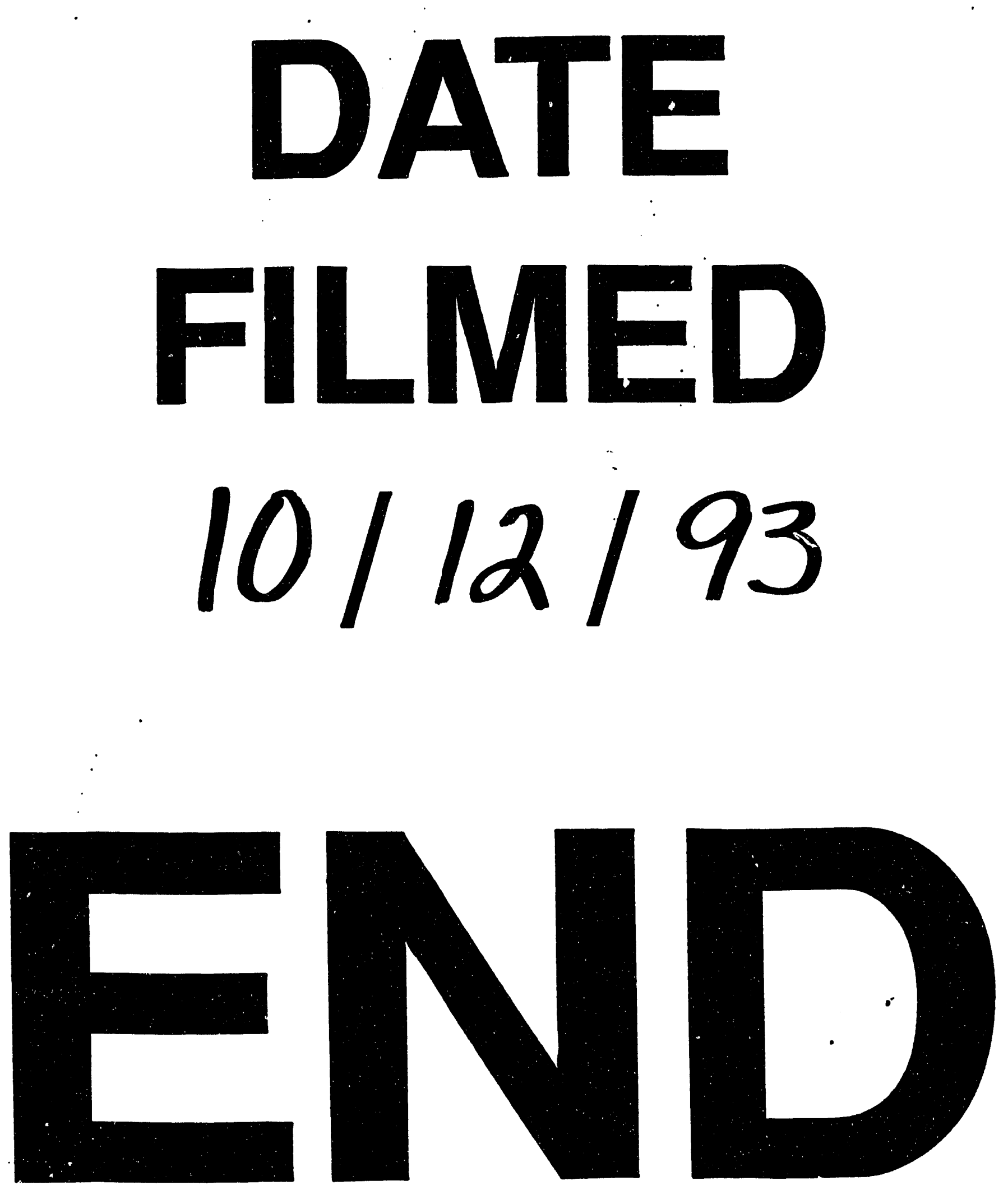
